\title{
An investigation of the role of paramedics in child protection
}

\section{Brady, James M.}

How to cite:

Brady, James M. (2018) An investigation of the role of paramedics in child protection. Doctoral thesis, Swansea University.

http://cronfa.swan.ac.uk/Record/cronfa46061

Use policy:

This item is brought to you by Swansea University. Any person downloading material is agreeing to abide by the terms of the repository licence: copies of full text items may be used or reproduced in any format or medium, without prior permission for personal research or study, educational or non-commercial purposes only. The copyright for any work remains with the original author unless otherwise specified. The full-text must not be sold in any format or medium without the formal permission of the copyright holder. Permission for multiple reproductions should be obtained from the original author.

Authors are personally responsible for adhering to copyright and publisher restrictions when uploading content to the repository.

Please link to the metadata record in the Swansea University repository, Cronfa (link given in the citation reference above.) 


\section{An investigation of the role of paramedics in child protection \\ James Michael Brady}

Submitted to the University of Wales Swansea in fulfilment of the requirements for the Degree of Doctor of Philosophy

Swansea University

2018 


\section{Abstract}

In the United Kingdom (UK), revelations of child abuse on an unprecedented scale have led to calls for better detection methods. Paramedics are invited into private households and are uniquely positioned to assist with this. This study aimed to explore the role that paramedics may play in child protection with a view to informing paramedic policy, education and practice.

Literature contains few empirical studies on paramedics and child protection. Legislation and policy documents acknowledge their potential contribution, but data is scant. Research is needed on their actual involvement, level of knowledge and skills. This study, undertaken in one UK ambulance service, employed a sequential mixed method design of two phases. In Phase 1, paramedics ( $\mathrm{N}=276$ ) completed a survey on their experiences, perceptions and attitudes. The resulting data informed Phase 2: paramedic focus groups $(\mathrm{N}=7)$. Discussions were thematically analysed. Carper's 'ways of knowing' was used as a theoretical framework throughout.

Findings suggest paramedics understand they have a key role to play, but lack clarity over the role. Although confident in their knowledge of physical abuse and neglect, they are less confident in their knowledge of sexual abuse (SA), childhood sexual exploitation (CSE) and female genital mutilation (FGM). More than half of respondents felt child protection referrals had not been made, that should have been. Barriers to safe practice include limited access to 'expert' led training; lack of feedback from social services, and lack of time.

Decision making is heavily dependent on intuition. Closer working relationships between paramedicine and social services are needed. Timely bi-directional communication is vital. Training input from social services is needed if paramedics are to develop greater confidence in detecting SA, CSE, and FGM. Finally, paramedicine needs to reflect further on the challenges that can arise in prioritising the best interests of children when working with families. 


\section{Declarations and Statements}

\section{DECLARATION}

This work has not previously been accepted in substance for any degree and is not being concurrently submitted in candidature for any degree.

Signed (candidate)

Date

\section{STATEMENT 1}

This thesis is the result of my own investigations, except where otherwise stated. Where correction services have been used, the extent and nature of the correction is clearly marked in a footnote(s).

Other sources are acknowledged by footnotes giving explicit references. A bibliography is appended.

Signed

(candidate)

Date

\section{STATEMENT 2}

I hereby give consent for my thesis, if accepted, to be available for photocopying and for inter-library loan, and for the title and summary to be made available to outside organisations.

Signed (candidate)

Date 


\section{Table of contents}

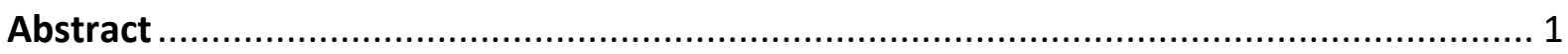

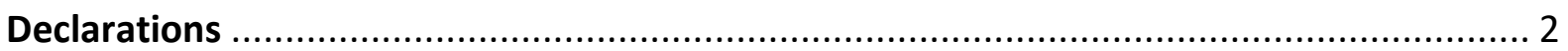

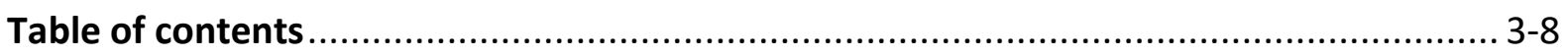

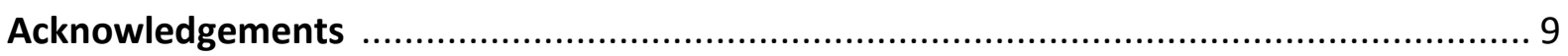

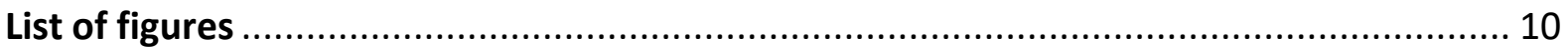

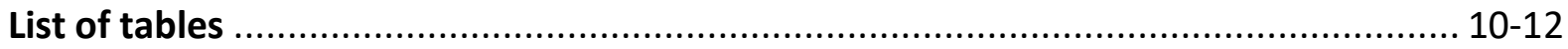

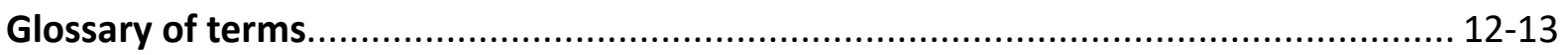

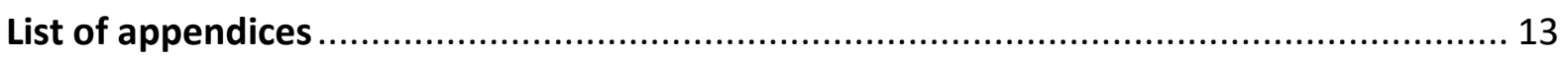

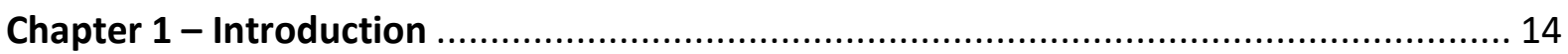

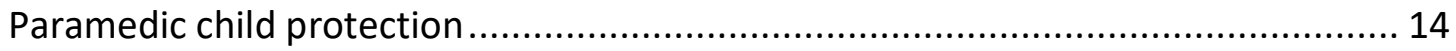

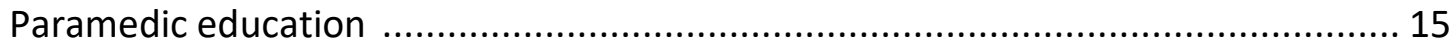

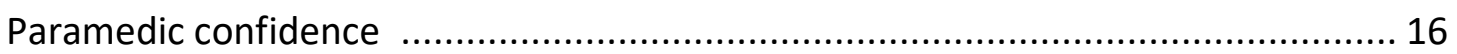

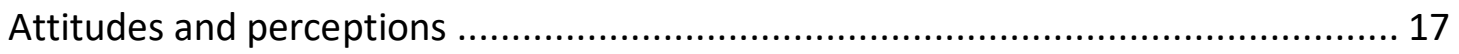

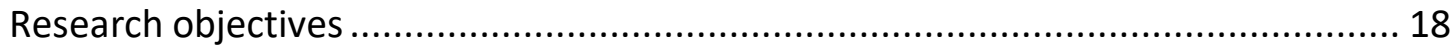

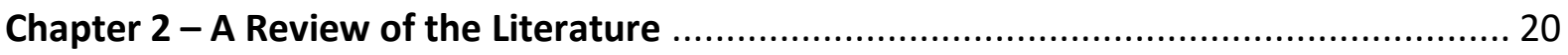

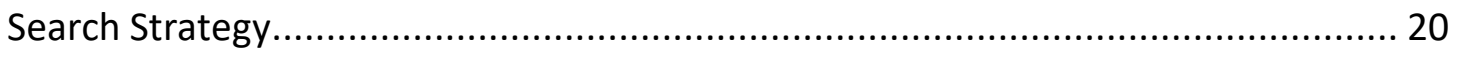

Paramedic interaction with children and young people ..................................... 24

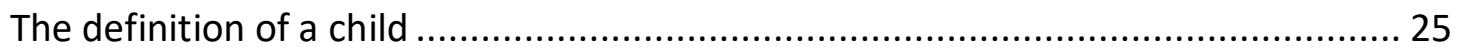

The definition of child abuse and neglect....................................................... 27

Cultural and religious factors affecting the definition of abuse and neglect ............. 29

Different types of abuse and neglect ............................................................ 31

The prevalence of abuse neglect and child death within the UK ........................... 64

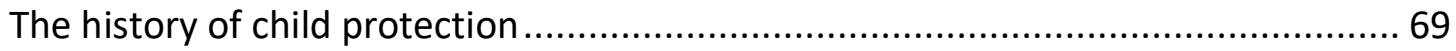


Reforming the UK child protection policy.....

Child Protection: Paramedic training and education

The psychosocial aspects of paramedic practice ............................................ 82

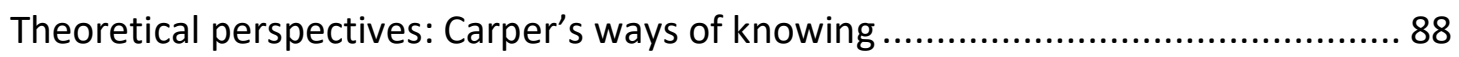

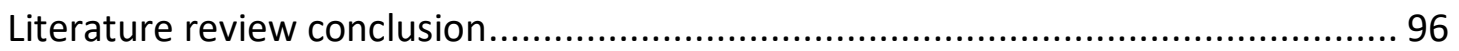

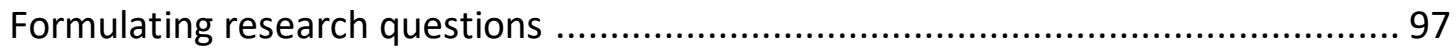

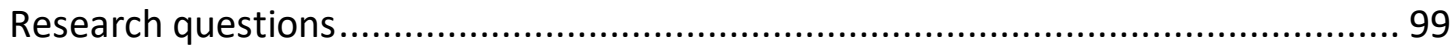

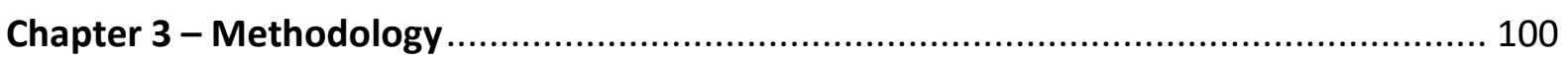

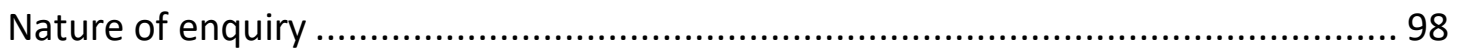

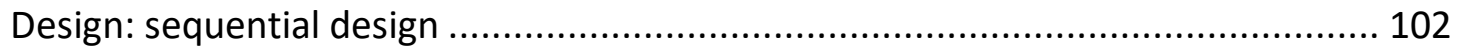

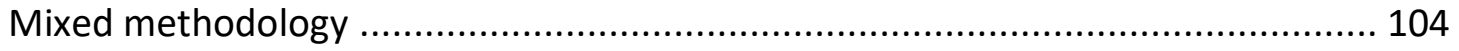

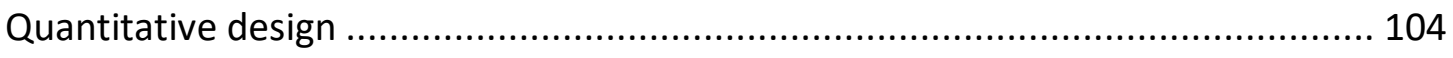

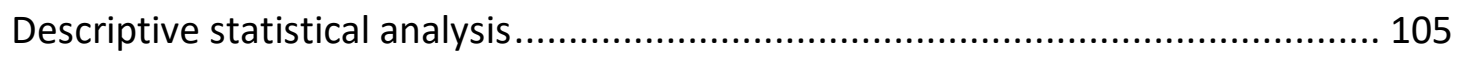

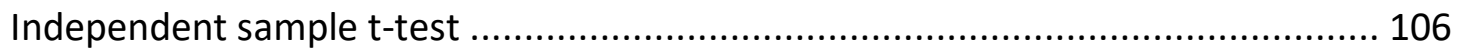

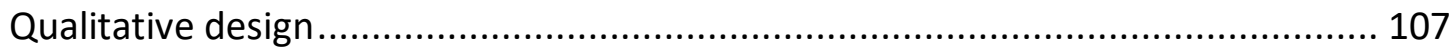

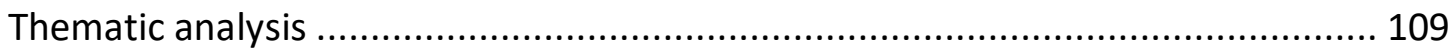

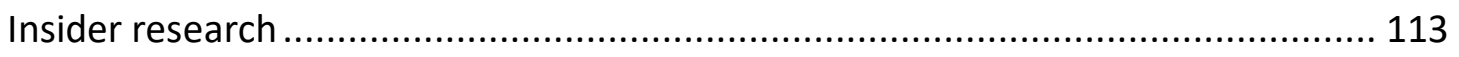

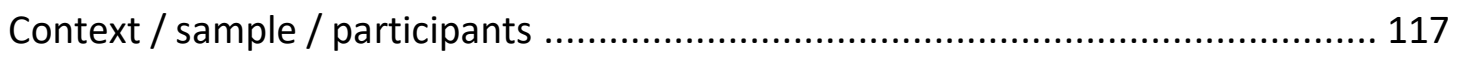

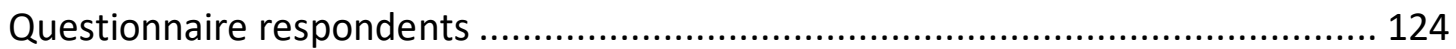

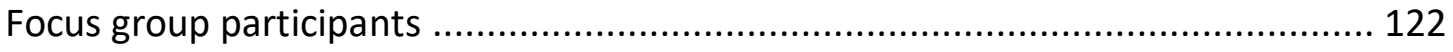

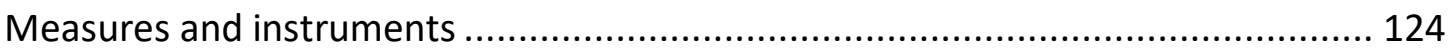

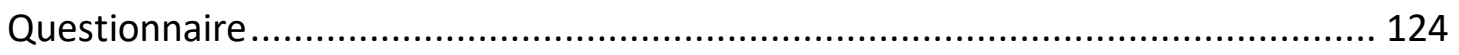

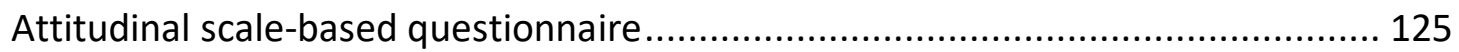

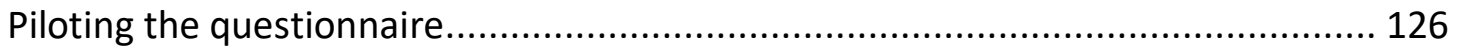

Pilot study one

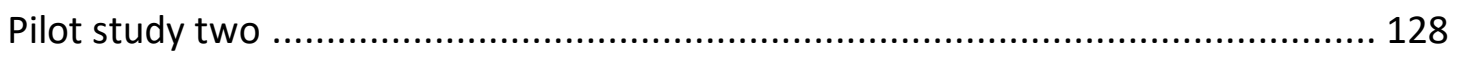




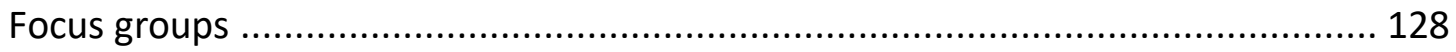

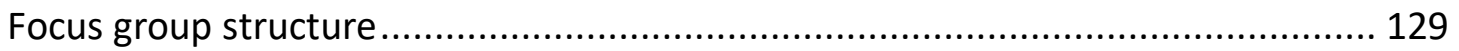

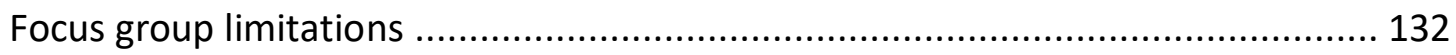

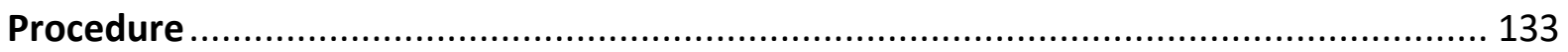

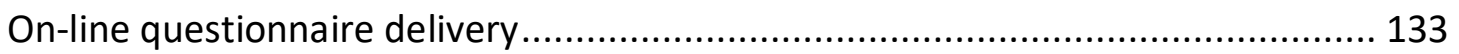

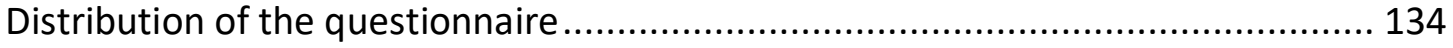

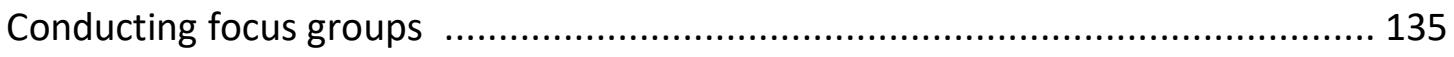

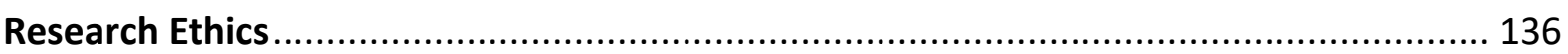

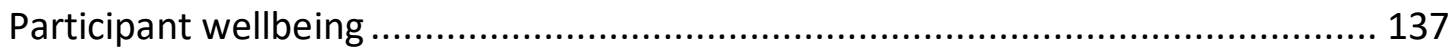

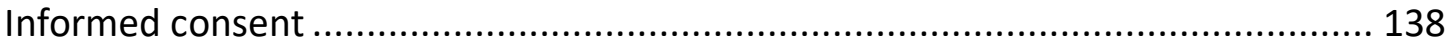

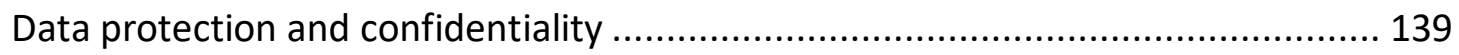

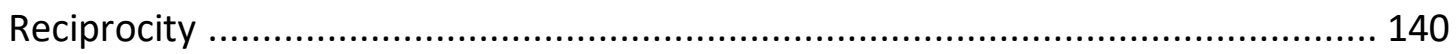

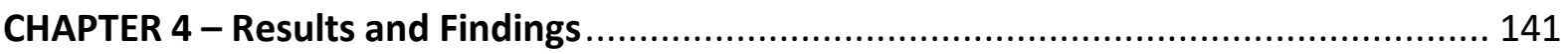

Question 1: How do paramedics perceive their role in child protection? ..................... 142

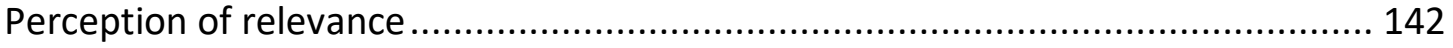

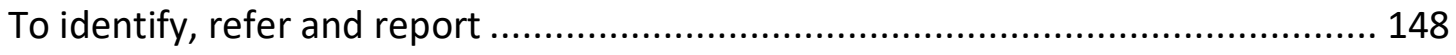

The Paramedics' position within the community and society.............................. 151

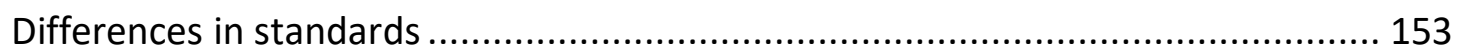

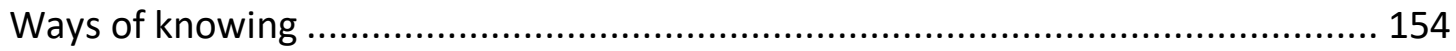

Question 2: How do paramedics perceive the training they receive on child protection? ...160

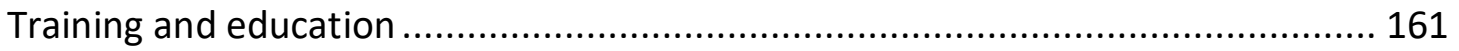

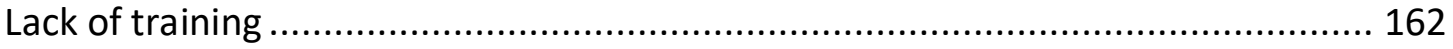

Experience-based and university-based learning ............................................. 166

Question 3: How confident are paramedics in child protection? ................................. 168

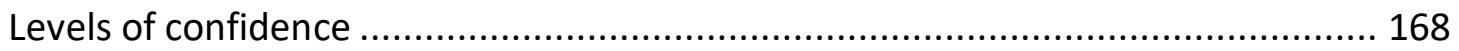

Lack confidence in recognising signs of sexual abuse, CSE, and FGM? ................. 174 
Non-physical signs of sexual abuse

Lack of training in sexual abuse, FGM, and CSE

Question 4: What barriers exist to paramedics undertaking their role in child protection? ...179

Lack of feedback 179

Being taken seriously 181

Reasons for not submitting child protection referrals 183

Lack of time or permission 186

Technology difficulty 187

Parental consent 188

Question 5: Can any ways of improving paramedic's practice in child protection be identified?

Electronic Patient Clinical Record 189

Scenario-based training delivered by experts outside the ambulance service......... 191

Peer review and colleague support.

Summary of findings. 194

Chapter 5 - Discussion 196

Question 1: How do paramedics perceive their role in child protection? 198

Question 2: How do paramedics perceive the training they receive? 204

Question 3: How confident are paramedics in child protection? 208

Question 4: What barriers exist to paramedics' undertaking $s$ their role in child protection? ...217

Barrier 1: Only realised in hindsight 218

Barrier 2: Not enough time or not allowed to return to station to complete referral ...220

Barrier 3: Poor facilities 221

Barrier 4: Lack of proof 223

Barrier 5: Lack of feedback 225 


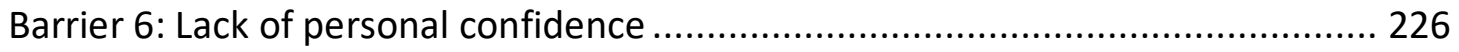

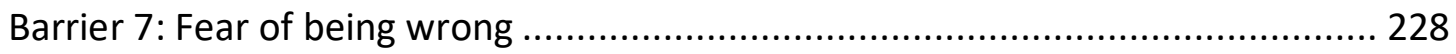

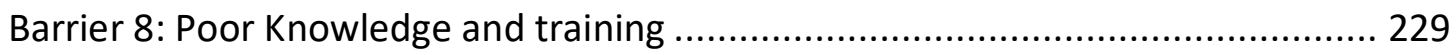

Question 5: Can any ways of improving paramedic's practice in child protection be

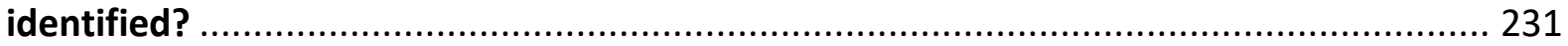

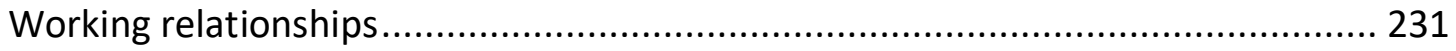

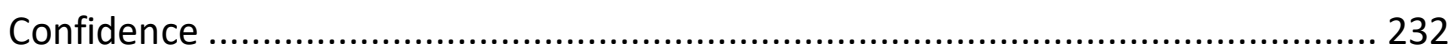

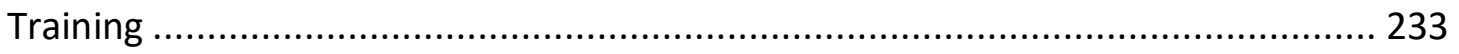

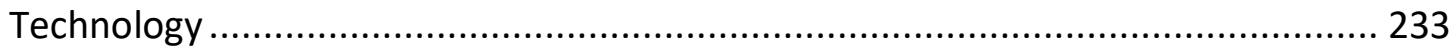

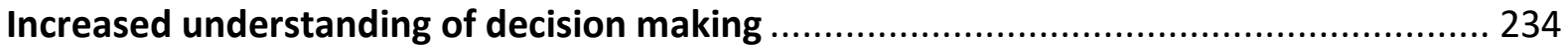

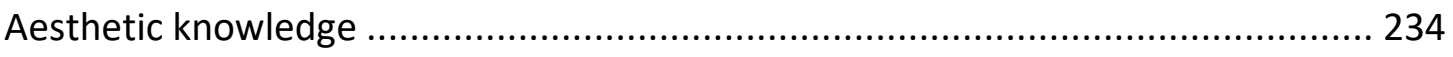

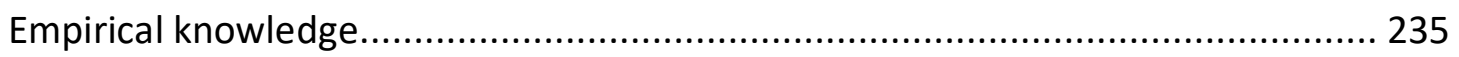

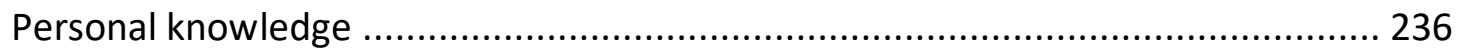

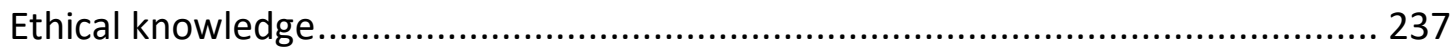

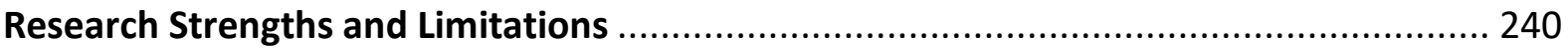

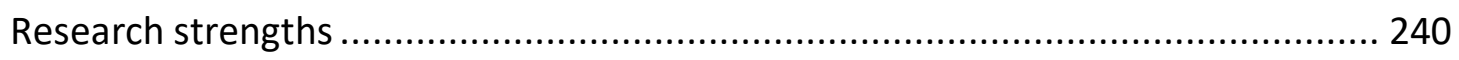

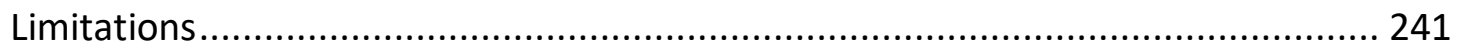

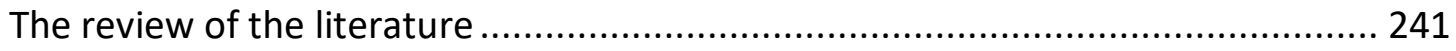

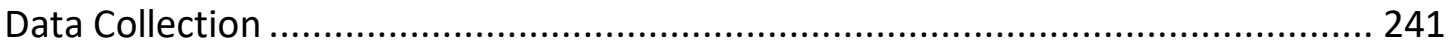

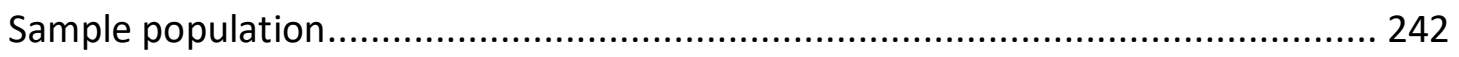

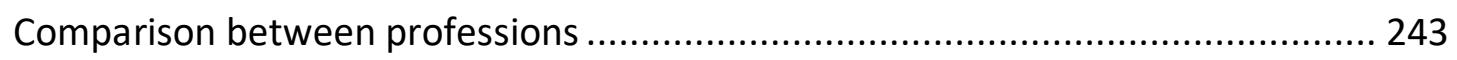

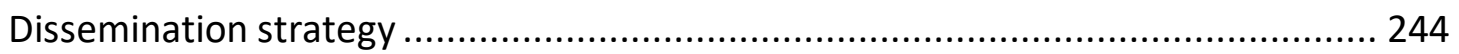

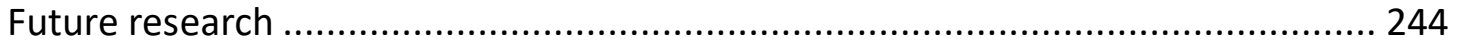

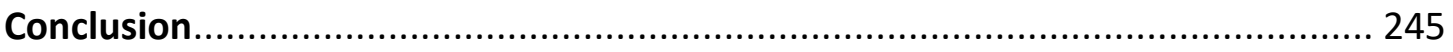

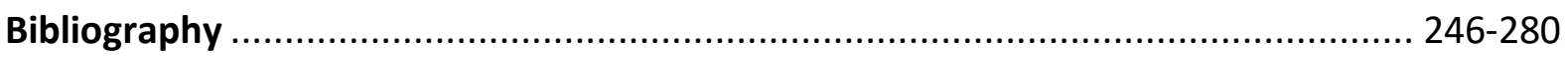

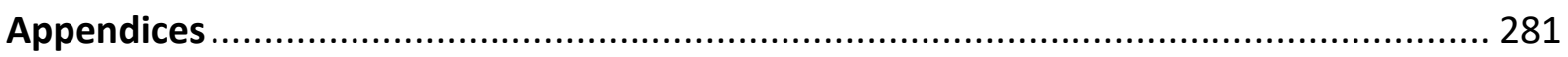

Appendix 1: Landmark UK cases ....................................................... 281-282 
Appendix 2: Notes of salient points during focus group - example

Appendix 3: Thematic coding notes from focus group - example

Appendix 4: Questionnaire (Full) $285-294$

Appendix 5: Advertisements for questionnaire participation 295

Appendix 6: Information sheet for focus group participants

296-298

Appendix 7: Consent form for focus group participants 299 


\section{Acknowledgements}

I would like to acknowledge and thank all those who have helped me along this academic and personal journey; from subject experts to friends, and those who have become both.

I would like to dedicate this thesis to the memory of William Patrick Brady (19602012), without whom, alongside the support of Teresa Brady, none of this would ever have been possible.

\section{Mum \& Dad}

I would like to acknowledge and thank my academic supervisors, namely, Dr Ruth Davies, Dr Paul Rees, and Dr Darren Edwards.

Thanks are due to Dr Ruth Davies for her belief in my abilities, research ideas, and subject area from the very beginning, as well as her ongoing guidance and continual supply of coffee and chocolate biscuits; to Dr Paul Rees for his ever-objective guidance, patience, and shared excitement in my ideas and prospects; and to $\mathrm{Dr}$ Daren Edwards for his mastery in all things statistical, mathematical, and quantifiable, but most importantly, for his patience in my development of such skills. Thank you.

I would like to acknowledge and thank all of those paramedics who took part in this research and have helped to provide a better understanding of child protection.

Last, but certainly not least, I want to thank my wife and best friend, Sarah. Without her unwavering patience, love, and ability to humour my excited, eccentric, and at times, unstable academic ramblings, this thesis would not have been possible. Her support and guidance has gone beyond that which could be reasonably expected and has seen me through significant academic and personal struggles throughout this journey. In doing so, and as a paramedic herself, she has also contributed to ensuring paramedicine continues to be a research-based profession.

Thank you. 


\section{List of figures}

Figure 1: Chart showing rapid review process, decision points and outcomes ...................... 23

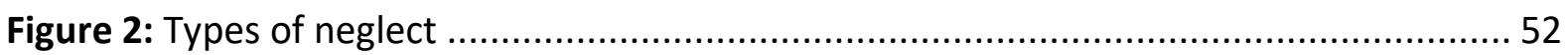

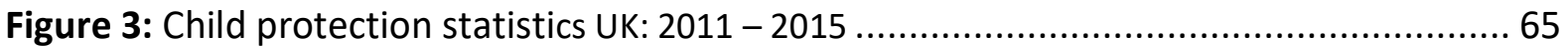

Figure 4: Numbers of deaths reviewed and percentage with modifiable factors by category of death

Figure 5: Social determinants of health 84

Figure 6: Diagram showing the relation between sociology, psychology, and physiology .... 86

Figure 7: Ways of knowing of paramedics 239

\section{List of Tables}

Table 1: Gender frequency of questionnaire respondents.

Table 2: Age range of questionnaire respondents

Table 3: Educational and career profile of questionnaire respondents

Table 4: Years of qualification of questionnaire respondents 120

Table 5: Years of service of questionnaire respondents

Table 6: Roles of questionnaire respondents

Table 7: Demographics of focus group participants

Table 8: Relevance of knowledge of physical abuse

Table 9: Relevance of knowledge of emotional abuse

Table 10: Relevance of knowledge of sexual abuse

Table 11: Relevance of knowledge of neglect 
Table 12: Relevance of the knowledge of psychological and sociological factors affecting child and family health

Table 13: Relevance of knowledge of health promotion \& disease prevention

Table 14: Relevance of the knowledge of lifespan development

Table 15: Independent sample t-test comparing the sex of respondents with respondents perceived relevance of knowledge of types of abuse

Table 16: Independent sample t-test comparing the length of total years' of service with respondents perceived relevance of different types of abuse

Table 17: Independent sample t-test comparing the years of qualified practice with respondents perceived relevance of different types of abuse.

Table 18: Use of factual knowing 155

Table 19: Use of aesthetic knowing 155

Table 20: Use of personal knowing. 156

Table 21: Use of ethical knowing 156

Table 22: Perception of child protection training 161

Table 23: Latest child protection update 161

Table 24: Levels of confidence in ability to recognise signs of physical abuse 168

Table 25: Levels of confidence in ability to recognise signs of emotional abuse 168

Table 26: Levels of confidence in ability to recognise signs of neglect...... 169

Table 27: Levels of confidence in ability to recognise signs of the effects poor housing have on child development 169

Table 28: Levels of confidence in their ability to recognise signs of the effects poor levels of social interaction can have on child development 170

Table 29: Levels of confidence in their ability to recognise signs of the effects that poor diet has on child development and educational attainment 
Table 30: Levels of confidence in their ability to recognise signs that a child may not be achieving their expected physical, cognitive, and psychosocial landmarks

Table 31: Independent sample t-test comparing the current role of respondents with their perceived relevance of different types of abuse

Table 32: Levels of confidence in their ability to recognise signs of sexual abuse

Table 33: Levels of confidence in ability to recognise signs of child sexual exploitation...173

Table 34: Levels of confidence in ability to recognise signs of female genital mutilation...173

Table 35: The number of child protection referrals made in the last three years 180

Table 36: Frequency of feedback on referrals 180

Table 37: Perception that referrals are taken seriously 182

Table 38: Frequency of referrals not submitted 184

Table 39: Reasons why referrals were not submitted 185

\section{Glossary of terms}

AACE: Association of ambulance service chief executives provides ambulance services with a central organisation that supports, coordinates, and implements nationally agreed policy. It also provides the public and other stakeholders with a central resource of information about NHS ambulance services.

CAD: Computer Aided Dispatch tools are used by ambulance services to record information, dispatch ambulances, and store warning markers on addresses.

CSE: Child sexual exploitation is a form of sexual, emotional, and physical abuse of children.

ECA: Emergency Care Assistant is an ambulance staff member less qualified than a paramedic, whose job it is to support the paramedic with whom they work.

FGM: Female genital mutilation, also known as female genital cutting and female circumcision, is the ritual cutting or removal of some or all of the external female genitalia. It is illegal in the United Kingdom.

HCPC: The Health and Care Professions Council is a statutory regulator of over 344,000 professionals from 16 health and care professions in the United Kingdom. 
IHCD: The Institute of Health and Care Development was a private healthcare provider who used to provide modular-based ambulance service and paramedic training.

JRCALC: The Joint Royal Colleges Ambulance Liaison Committee, whose role is to provide robust clinical speciality advice to ambulance services within the UK.

Paramedic: A HCPC-registered healthcare professional, predominantly, but not exclusively, working in the pre-hospital and out-of-hospital environment for ambulance services.

SCR: Serious Case Reviews are carried out when a child dies or is seriously harmed, because of abuse or neglect. They are designed to identify ways that local professionals and organisations can improve the way they work together to safeguard children.

Stood down: Being stood down refers to an ambulance crew being marked as unavailable to attend emergencies by their dispatcher. An ambulance crew can be stood down at the end of their shift, to deep-clean an ambulance, or attend an important meeting.

\section{List of Appendices}

Appendix 1: Landmark UK cases

Appendix 2: Notes of salient points during focus group - example............................... 283

Appendix 3: Thematic coding notes from focus group - example

Appendix 4: Questionnaire (Full) 285-294

Appendix 5: Advertisements for questionnaire participation 295

Appendix 6: Information sheet for focus group participants. 296-298

Appendix 7: Consent form for focus group participants 299 


\section{Chapter One: Introduction}

In late 2011, the United Kingdom (UK) discovered that charity worker and television personality Jimmy Savile had allegedly committed hundreds of sexually abusive acts during his celebrity career. This revelation sparked many allegations and investigations into historical abuse concerning individuals from organisations such as the British government, the British Broadcasting Corporation, and the Roman Catholic Church. In addition to allegations surrounding sexual abuse, the UK health and social care services were coming under increasing scrutiny after multiple child death cases, which were deemed repetitive and in which previous lessons had not been learned. Such lessons included the need for health and social care professionals to be suitably trained, supervised, and updated on child protective practices. These high-profile inquests and investigations subsequently forced many organisations and professions across the UK to critically reflect upon their child protection history and current practice. Paramedicine was one such profession.

I began my career as a graduate paramedic having qualified from Swansea University on the first undergraduate paramedic science course in Wales. In 2012, having been qualified several years and following revelations of child abuse from serious case reviews, I felt there was a real need for paramedicine to question its own role in child protection critically. This desire was strengthened by my own experiences as a Clinical Team Leader and an associate lecturer in emergency and unscheduled care which revealed a lack of focus within the paramedic educational syllabus on child protection. Bearing all this in mind I decided to undertake $\mathrm{a} \mathrm{PhD}$ to research the role of paramedics in child protection.

\section{Paramedic child protection}

Paramedics are granted access into homes and environments that many other professions are not, and can witness family life, often at the point of crisis. They can be called to assess and treat children directly or can encounter children indirectly through the assessment and treatment of patients with children (Brady, 2012). They are often the first point of contact for children and young people in need for acute assessment and treatment (JRCALC, 2017) 
and so have an integral role in child protection. Paramedics need to record and share their findings and concerns with other professionals who do not have such unrestricted access. This identification, recording, and sharing, aids early intervention and support, and at times, leads to the removal of children to safety. Despite this, however, it is challenging to determine just how many children paramedics encounter in their everyday practice. There is no single compendium of figures relating to children and young people using 999 services. It is also not known how paramedics themselves perceive and understand their role in child protection or how confident they are in carrying it out. Furthermore, it is not known what training paramedics are given to carry out this role, or indeed, if they even want to undertake it. While it is true that many UK paramedics work in schools, GP surgeries, and minor injury units, most work for UK ambulance services, responding to emergency 999 calls. This requires both the profession itself and employers to reflect upon past and current child protection practices and to explore the efficacy of current and future practice.

\section{Paramedic education}

Paramedics, like all health care professionals (HCPs), require sufficient education to underpin their practice. This education has changed in line with the changing roles of paramedics over the last 30 years. Paramedics are still often mistakenly synonymised with the stretcher-bearing ambulance-driving first aiders of the 1960s, who respond only to lifethreatening situations of trauma or resuscitation. The modern paramedic's education, however, is underpinned by university degrees at undergraduate, masters, and doctorate level. These qualifications enable the provision of autonomous community-based biopsychosocial care which does not involve taking every patient to hospital and which involves the care of chronically ill patients also. This care inherently includes the assessment and treatment of children and young people. The late 1990s saw the education of paramedics start to represent their workload better and include in-depth, evidence-based anatomical, physiological, pathophysiological, and pharmacological education. Importantly, however, in response to the true paramedic workload, subjects such as health promotion, disease prevention, mental health, socioeconomics, and child protection were also included in varying amounts. The inclusion of these subjects recognised the wide role paramedics 
play within the community, not least in child protection. Nonetheless, to what extent this education prepares paramedics for their role in child protection is unknown. This move from a vocational role to basic medical training, and more recently to higher education, introduces the concept of different 'ways of knowing'. Originally conceived by Carper (1978), this theory expands upon traditional ideas of empirical theory to encompass more inclusive and diverse ideas of knowledge, such as aesthetic knowing, ethical knowing, and personal knowing. It is theorised that by exploring these different ways of knowing, a deeper level of understanding can be gained by an individual and a group. This theory will become more prominent throughout this thesis and is integrated into any roles paramedics have in child protection and the education that underpins it.

\section{Paramedic confidence}

Health care practitioners should have confidence in their ability to carry out any given role for which they have responsibility. Self-confidence has been defined as the belief in one's ability to accomplish a goal or task (Potter \& Perry, 2001) and has been reported as being a key component for effective clinical competency and performance (Porter et al., 2013). This suggests that the confidence paramedics have in their abilities is likely to affect the quality of their work. The Millar report (1966), which influenced the education and training of ambulance staff, highlighted the need to give "ambulance crews the sufficient background knowledge to be able to make certain decisions with confidence" (Ministry of Health and Scottish Home and Health Department, 1966 p. 7). Previous studies have suggested that professionals' lack of confidence in reporting and their misguided beliefs about child abuse and neglect are a result of a lack of training (Raman et al., 2012). Adequate education and training, however, are only two contributing factors to high confidence levels. Selfconfidence can also be indicative of organisational trust, adequate support, good mentorship, effective management, and a culture of learning (Azaare and Gross, 2011; Porter et al., 2013; Papathanasiou et al., 2014; Kim et al., 2014).

It could be argued that a professional history and a strong professional culture contribute to individual and organisational confidence levels. In comparison with other professions, such as medicine or nursing, paramedicine has lacked a professional history and is sometimes 
viewed as a hybrid of knowledge and skills that are taken from other pre-established professions (Campeau, 2008). Determining how confident paramedics are in their role of child protection can, to a certain extent, determine the quality of their work. Moreover, determining how confident paramedics are can indicate a great deal about the profession and organisation in which they carry out their role; and can help to determine if any improvement strategies are required. Munro (2011) argues that child protection workers need confidence in their own skills and knowledge, as child welfare is arguably one of the most complex and challenging areas of work. Reporting concerns alone does not equate to having knowledge and confidence in identifying abuse and neglect (Raman et al., 2012). For example, deciding not to report or refer a child or family can equally indicate knowledge and confidence in child protection, thus highlighting how complex a notion confidence can be in practice. Given that the development of self-confidence is central to better patient outcomes, effective performance (Hayes, 2003) and the overall learning process (Smyth and Long, 2011) it is important to explore this throughout any review of the literature regarding paramedics and child protection. Confidence in any given area of practice may be influenced by the practitioners' attitudes and perceptions towards it, and therefore, this will also be explored throughout this research to determine to what extent paramedics' attitudes may influence their ability to carry out their role.

\section{Attitudes and perceptions}

'Attitudes' refers to a person's tendencies to evaluate entities with favour or disfavour (Eagly \& Chaiken 2007). Attitudinal theory posits that individuals' values, beliefs, and attitudes lead to intentions that will then determine their behaviour (Fishbein \& Ajzen 1975). Thus, by exploring the attitudes of a group of people, one can also explore the possible behaviours of such a group, such as paramedics' attitudes and behaviours towards child protection. Child protection workers' attitudes towards responsibility, for example, can affect various decision thresholds (Davidson-Arad and Benbenishty 2010). This suggests the possibility that different attitudes may cause different and inconsistent approaches to child protection, which are influenced by emotion, previous experience, and bias. Whilst investigating paramedics' role in child protection, one must also investigate if paramedics 
themselves want a role or even feel that they have one. Nikolova et al. (2017) argue that understanding child protection workers' attitudes about responsibility for child safety is an important feature of developing effective services for children and families. Indeed, responsibility for child safety may be influenced by various factors and may have a significant impact upon the decisions that child protection workers make. Furthermore, it has long been recognized that employees' job attitudes can have implications on performance (Schleicher et al., 2015). Performance usually involves deadlines, sales targets, or project objectives. Performance for those who work in child protection, however, can relate to the lives of children and families and their ability to thrive. Attitude is only one of many factors that might influence decision making or perception towards child protection, but given that it might affect the decision-making process, professional behaviours, and performance, it is an important concept to explore as part of this research. This research will critically question these elements of confidence, training, and attitude, and investigate which types of knowledge inform paramedic decision-making in child protection. The overall objective of this research thesis is to inform future paramedic practice, education, and policy.

\section{Research Objectives}

Specific research objectives help to divide research into distinct sections and help address each part separately. Such objectives drive all aspects of the chosen methodology including instrument design, data collection, analysis, and ultimately, any suggestions that may result from the research. In general, research objectives describe what we expect to achieve by a project.

The specific objectives of this research are:

- to examine the perceptions paramedics have of their role in child protection

- to examine how confident paramedics are in child protection

- to examine how paramedics perceive the training they receive in child protection

- to identify any barriers to paramedics undertaking their role in child protection

- to identify ways of improving paramedics' practice in child protection 
Chapter 1 of this thesis has provided the context in which this PhD was undertaken and provided information on the roles of UK-based paramedics. It has highlighted how they work within the community with children and young people and have access that others do not. It has questioned how paramedics perceive this role in child protection and how confident they are in practice. To better understand this role and to inform future paramedic practice, policy, and education, Chapter 2 critically explores the current literature and investigates the prevalence of research within this area of paramedic practice. Based upon these findings Chapter 3 sets out the methodology required to investigate this further and contribute original research to the literature. Chapter 4 reports the results of this research, and Chapter 5 discusses the research and its implications for current and future practice. Chapter 6 concludes the thesis and sets out suggestions for future paramedic education, policy, and practice to better prepare paramedics to recognise the need to protect children, young people, and families. Paramedics work in diverse areas of clinical practice, but still work predominantly within the community for UK ambulance services. More paramedics are achieving masters and doctoral level degrees (Jones et al. 2010) and are contributing to original research. Given the recent revelations about widespread institutional and individual child abuse within the UK, it is important to understand the interplay between the changes in the paramedic profession and paramedics' responsibilities to protect children and young people, about which little is known. 


\section{Chapter Two: A Review of the Literature}

In the previous chapter, the rationale of this research was introduced, and the history of the ambulance service and paramedics was explored as the context for this study. In this chapter, the role paramedics play in the protection of children and young people is established through a systematic review and appraisal of the literature. A systematic review of the past literature is described by Levy and Ellis (2006) as a crucial endeavour for any academic researcher, the importance of which should not be underestimated. A review of the literature can lead to the identification of gaps and inconsistencies in a body of knowledge, thus justifying the need for further research, identifying research questions, and at times, pointing to certain methodological approaches (Cronin et al. 2008). Both theoretical and practical progress can be hindered if this process is not completed properly; hence the need for a systematic approach.

\section{Search Strategy}

A systematic review of the literature was undertaken by the researcher using the key terms "Paramedic", "Emergency Care Practitioner", "Emergency", "Ambulance”, "Pre-hospital”, "Safeguarding", "Protection", "Abuse", "Neglect", “Children" "Young people", "Psychosocial", and "Social". These terms were entered individually and in combination to the CINAHL, PubMed, Science Direct, and Cochrane databases. Paramedic Practice journals, Department of Health (DoH), and House of Commons annals were also searched. No date criteria were set, as the author deemed the historical progression of paramedic education and safeguarding practice to be integral to this research. Only literature of the English language was included, and articles were excluded if they were referring only to safeguarding vulnerable adults but did not mention children and young people. Articles from countries other than the UK but which follow the 'Anglo-American Model of Emergency Care' were included. The Anglo-American model of emergency care is one of two models that has influenced much of the worlds pre-hospital care delivery. The AngloAmerican model involves bringing the patient to the doctor. This is a paramedic-based system where out of hospital care is delivered by paramedics who then transport the 
patient to the hospital-based emergency department for continued and advanced care. In contrast, the Franco-German model involves taking the doctor to the patient. The physicians and the technology attend at the scene and provide a higher level of emergency care before a patient's arrival at hospital (Page et al., 2013; Evans et al., 2012). The Anglo-American model places the overall responsibility and duty of care firmly with the paramedic and not a more senior clinician such as a physician. The countries that conform to the Anglo-American model (USA, Canada, Hong-Kong, South Korea, Iran) have similar educational frameworks, scope of practices, and registration frameworks and requirements to UK paramedics; thus, any literature found may be adaptable and valid to inform the formation of research questions.

During the early stage of this literature review, it became evident that the paramedics' role in protecting children was not well represented. Results which referred to emergency nursing were also considered for review if they referred to child protection and not solely to the nursing profession or children's nursing emergencies. Although accident and emergency nursing is considered a separate profession, which takes place within a separate working environment, there are similarities between the way both professions work and practice, especially as front-line practitioners. Both professional groups see people at the point of crisis and have to assess the veracity of how injuries and illness were caused. Furthermore, if children are presenting to emergency nurses in accident and emergency departments, then paramedics may have been responsible for their transport and clinical care until patient handover, further showing the relevance of any possible articles found.

Paramedics do not solely work for publicly funded ambulance services; they can be employed in a range of roles in both public and private industry. Ambulance services, however, remain the largest employer of paramedics in the UK, and it was thought that literature from ambulance services would also be pertinent to this review. This literature is often termed 'grey literature'. Grey literature is described by Hopewell et al. (2007) as literature that has not been formally published, such as abstract reports, personal communications, education policies, referral procedures, and call data. Such literature can 
aid the investigation of paramedics' role in protecting children and young people from an employment perspective - through the review of safeguarding policies, guidelines on treating non-mobile injured children, and referral pathway documents. While it has been argued that such literature is often of poor methodological design, Franks et al. (2012) assert that with an adequate appraisal of such evidence, it can form acceptable evidence bases. 
Figure 1. Chart showing rapid review process, decision points, and outcomes.

Svstematic search with inclusion $\&$ exclusion criteria applied

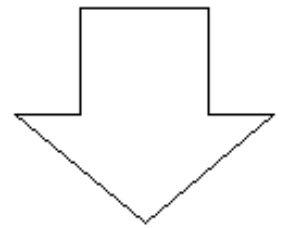

Results identified: 105

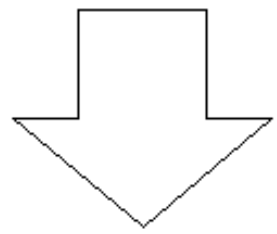

Abstracts reviewed for inclusion and exclusion criteria

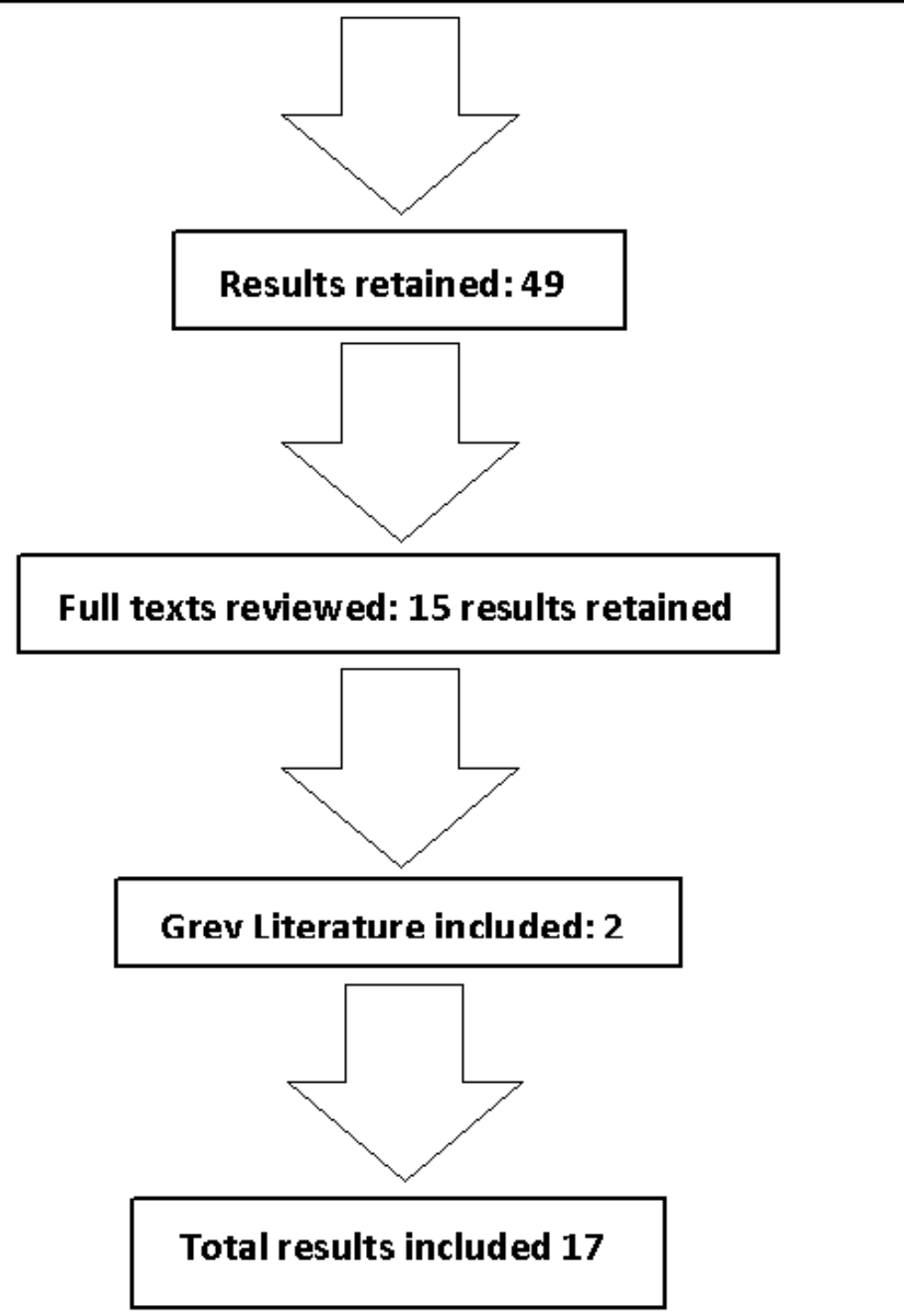


17 literature results met the inclusion criteria. Other sources were used more generally which can be adapted to paramedic practice; however, that these results have not been replicated in paramedic practice or do not include paramedic practice is noteworthy.

This chapter provides an overview of the literature about the role paramedics play in protecting children and young people. It commences by reviewing the number of children paramedics may encounter in practice and critically examining what is meant by the term 'child', both conceptually and legally. Attention then turns to the different types of abuse and neglect and the paramedic's role in identifying them and referring their concerns. The chapter then investigates any literature surrounding these types of abuse and paramedic practice.

\section{Paramedic interaction with children and young people}

Due to the nature of paramedic practice, it is difficult to determine fully the number of children and young people paramedics encounter each year. This difficulty lies initially with the lack of any single compendium of figures relating to children and young people using 999 services. Recent figures, however, indicate that children and young people are more frequent users of Accident and Emergency (A \& E) than adults. In 2015/16, there were 425 A

\& E attendances for every 1,000 children and young people compared with $345 \mathrm{~A} \& \mathrm{E}$ attendances for every 1,000 adults aged 25 and over (NHS Digital, 2017). Paramedics may be responsible for the transport of these children and young people to hospital and thus the assessment and treatment of them beforehand. Whilst figures from $A \& E$ attendances go some way to showing the interaction paramedics may have with children, this is inherently limited. Sick children are more mobile than sick adults by virtue of their size, and so many children present to $A \& E$ by car and not by ambulance. Moreover, many patients, including children and young people, are assessed by 999 services over the phone by paramedics with no need for an actual ambulance attendance or hospital admission. Similarly, many patients are assessed and treated on scene by paramedics also without admission to hospital (AACE, 
2017); thus, various statistics would omit these patient groups. In addition, paramedics may encounter children and young people during interactions with adult patients, and so the use of patient age data might also be limited in determining interaction with children and young people. Whilst paramedics may have safeguarding concerns for these children, there may be no medical need at the time of interaction, so no electronic or paper patient care record form would be completed, only a safeguarding referral. These factors often mean that looking at the frequency of ambulance responses for children and young people alone would produce inaccurate data. Despite these limitations, however, it is recognised that there is potential for paramedics to encounter children and young people every day throughout their clinical practice. Paramedics are often the first healthcare professionals to have access to these vulnerable groups at the time of crisis when they are in need of support and referral (JRCALC, 2017) despite the initial reason for the call itself. The importance of this access cannot be underestimated and requires researchers to critically question how we define childhood, abuse, and neglect.

\section{The definition of a child}

Before the 17th century, while people were recognised as 'children', there was no such thing as 'childhood'. This lack of recognition was particularly reflected in clothing styles, approaches to education, and gender roles, such as household functions. The National Society for the Prevention of Cruelty to Children (NSPCC) was set up in 1884, some forty years after the creation of The Royal Society for the Prevention of Cruelty to Animals. The NSPCC (2012 A) states that in the UK, no single law defines the actual age of a child, but rather there are multiple sources that define a child within differing contexts. For example, the Children Acts (1989) and (2004) respectively use the definition that 'A child is anyone who has not yet reached their 18th birthday'. The Child Poverty Act (2010), however, defines a child as 'a person under the age of 16'. While these sources focus on the chronological age of a child, UNICEF (2014) refers to childhood as being much more than just the space between birth and the attainment of adulthood. UNICEF refers to the state 
and condition of a child's life and the quality of those years as having time to play, to grow confident, and to be free from fear, violence, and exploitation.

People in more developed countries have longer lasting childhoods with more time for educational, personal, and social development. People in less developed countries, however, must 'grow up' faster and have to contribute to community and family life through comparatively more rigid gender roles and social positions. Worldwide agreement on a definition of a child is unlikely to occur given these anthropological differences in race, religion, culture, and gender emphasis. Such differences often cause those researching and practising with children and young people much difficulty (Holzer and Broomfield, 2010). This difficulty also applies to paramedics, who may experience conflict between various acts of law. The Mental Capacity Act (2005), for example, applies to people over the age of 16 regarding consent to treatment and best interests, but co-exists with the Children Act (2004), which still considers the person to be a child at this age. This conflict can arise when paramedics are deciding upon sharing sensitive information, reporting child protection concerns, and reporting possible illegalities. This dichotomy introduces concepts such as 'age maturity', which itself is often confusing and fraught with conflicting theories on the indications of maturity, which appear to depend upon differing political agendas (Todres, 2012), such as education, health, and state-endorsed religion.

This conflict between what constitutes a child involves different ways of knowing in clinical practice. This theory was first introduced by Carper in 1978 and will become more prominent throughout this thesis as a theoretical framework. Carper's ways of knowing include traditional ideas of empirical theory, such as the child's chronological age, but move beyond traditional views to more inclusive and diverse ideas of knowledge, such as aesthetic knowing, ethical knowing, and personal knowing (Carper 1978), and how this is organised, tested, and applied in clinical practice. If, for example, empirical ages cause conflict over the definition of a child, questions then arise regarding how a paramedic comes to a decision regarding child protection and what knowledge they use to come to this decision. The confusion and complexity surrounding the definition of a child is well represented within the literature; however, little is known about the extent to which this 
affects paramedic practice. It could be argued that the point of defining a child may be entirely dependent upon the type of suspected abuse or neglect in question, and so the following section introduces the different types of abuse and neglect that paramedics are likely to encounter.

\section{The definition of child abuse and neglect}

The definitions of abuse and neglect are arguably somewhat clearer than the definitions of a child, but are not devoid of conflict and confusion. Unless paramedics working with children and young people have a clear definition of what constitutes the abuse and neglect of a child, they are unlikely to be able to identify those children and young people potentially at risk. From an international perspective, the World Health Organisation (WHO) (2016) defines the maltreatment of a child as follows:

All types of physical and/or emotional ill-treatment, sexual abuse, neglect, negligence and commercial or other exploitation, which results in actual or potential harm to the child's health, survival, development, or dignity in the context of a relationship of responsibility, trust, or power. Exposure to intimate partner violence is also sometimes included as a form of child maltreatment.

While both abuse and neglect can be carried out purposely, and with malicious intent, neglect can also result from socio-economic deprivation and an inability to cope for various reasons, which will be explored further. Recognising these differences is crucial for those working with children and young people, as the emphasis can be either one of protection of the child, or one of guidance, support, and financial assistance. Goddard and Tucci (2008) argue that early developments in the knowledge of the problem of child abuse have been accompanied by disagreements about such definitions, especially regarding theoretical approaches to causation, efficient approaches to practice, and the incidence of occurrence. No research investigating the knowledge of paramedics in relation to different types of abuse and neglect was identified within this review. Gaining consensus on the definitions of abuse and neglect has been historically problematic, and arguably, it continues to be problematic without sufficient investigation into how this affects clinical practice. Within the 
UK, there is no single piece of legislation that covers all areas of child abuse and neglect; instead, there is a series of Acts, which are continually being amended, updated, and revoked. This setup is not unique to the UK, but is mirrored in various countries across the West. This lack of uniformity has led to incoherent public policy approaches to protecting children and young people (Goddard and Tucci, 2008), which may be manifested in paramedic practice, although there is a lack of research investigating this within the literature. Slep et al. (2011) argue that in the case of emotional abuse, the lack of consensus on practice-based definition severely hampers the ability to improve the identification, prevention, and treatment of emotional abuse. It could be argued that such hampering can exist within paramedic practice and can involve other types of abuse; however, no research to support this assertion has been identified by this review.

This disparity in working definitions is not confined solely to practice, however, as it is also evident in academic research. Even within the same discipline, there is a debate about whether definitions should be based on the behaviour of the perpetrator, the experience of the child, or on some combination of the two. However, while legal definitions are essential to bring those guilty of abuse and neglect to justice, the limitations become evident when comparing the use of such definitions between law, academia, and the realities of clinical practice. Runyan et al. (2005) state that despite the need for clearer definitions among academics, the lack of consensus continues to be a challenge and inhibits a unified approach because of differences across different disciplines and how these definitions are used in law, medical social work, and psychology. Trickett et al. (2009) posit that fundamental problems can arise when psychologists or social scientists take definitions developed for legal or administrative purposes and use them to define constructs in research.

To combat this, there is a myriad of guidance documents to support these legal definitions. The differences in definitions and the differences in opinion may explain why there appears to be no universally agreed definition used in the paramedic-specific literature identified by this review or within the grey literature. This lack of uniformity may result in differences in what paramedics consider to be a child protection issue and the threshold of these issues, 
although there exists no research investigating this possibility. This lack of uniformity in definition can be further complicated when looking at different cultural practices within the UK, and the next section of this chapter explores this issue in more detail.

\section{Cultural and religious factors affecting the definition of child abuse and neglect}

Pierce and Bozalek (2004) state that in many countries and societies, child abuse is only now being viewed as a real problem, and practitioners working in the area have only just begun to rigorously compare how abuse and neglect are constructed in countries with diverse cultures. Various definitions of abuse focus on the interaction between the parents and the child. The cultural background of the family often informs this interaction, and as a result, conflict can arise if cultural norms to which the family conform oppose those of the society in which the child lives, and the laws (both morally and statutory) which must be obeyed. Pierce and Bozalek reviewed definitions of abuse and neglect from a South African perspective. Literature related to South Africa has been included within this review because of the Anglo-American model of emergency care followed. While it can be argued that the UK has had clear child protection policies for many decades (with differing degrees of efficacy), the UK population is becoming more culturally and religiously diverse, with the number of minority ethnic groups continuing to rise since 1991 (Office for National Statistics 2015). There have been cultural and religious factors attributable to child deaths in various serious case reviews, including the case of Victoria Climbié in 2000, which is now considered a landmark case for child protection in the UK due to the various recommendations for practice that resulted from the investigation. Climbié's death involved religious and cultural elements that were seen as contributory to her death, including her entry into the country, her parent's willingness to send her to the UK, and the role of evil spirits and demonic possession.

Korbin (1991) introduced three concepts when formulating culturally appropriate definitions of child abuse and neglect: cultural differences in childrearing practices and 
beliefs, idiosyncratic departure from one's cultural continuum of acceptable behaviour, and societal harm to children. Concepts such as these may assist paramedics to begin to understand and identify causal or acceptance factors of child abuse and neglect if included in the paramedic curriculum in higher education; of which there was no evidence found within this review. Archer and Spenser (2006), argue that there is a lack of understanding, education, and knowledge of diverse cultures within the paramedic education. Their review of the literature about Australian paramedic education was conducted to identify specific conventions and noteworthy initiatives which offered a foundation for the inclusion of cultural diversity in paramedic education, training, and practice. They argue that there is a lack of a readily accessible single compendium in the area of culturally responsive health care to be used in paramedic education or practice and that more education is required. The findings of this study have implications for child protection practice, and cultural differences have been identified as causal factors in serious case reviews. Although this research is now somewhat dated, a more recent study by Hartley (2012) draws similar conclusions.

Hartley's (2012) study used narrative enquiry and thematic analysis to identify common threads in responses from two independent volunteer groups comprising community groups $(n=20)$ incorporating representatives from the African, Asian, Middle Eastern, Muslim, Jewish and Indigenous Australian communities, and paramedic practitioners $(n=20)$. The purpose of the emergency call out and the satisfaction with the paramedic care were investigated using semi-structured focus groups and face-to-face interviews with the paramedic group. Hartley identified that the paramedics had a deficit of knowledge and a presence of incorrect knowledge about cultural practices that directly affect professional practice. He concluded that paramedics who received either minimal or no education relating to cultural or religious preparedness lacked sufficient knowledge pertaining to their patients' needs and may have had generalised and homogeneous views of nationalities and cultures, derived from personal experiences and childhood exposure. While this study was not specifically looking at child protection, its findings are valid and applicable to child protective practice. There was evidence that paramedics' inability to recognise certain customs led to false accusations of impropriety, which caused tension between them and 
their patients. Misconceived impropriety can result from the subservient gender-specific roles within households, which differ from those of Western culture, for example, asking permission from a husband for his wife to be medically assessed by a man. From a UK perspective, Haley's (2011) reflective case study reminds UK paramedics that in many cultures, it may not be the mother who is the primary attachment figure, as fathers and other family members can play a greater part in children's cognitive development. Thus, what might seem to be a distant mother and overly affectionate father in a western context, may perhaps be a normal family structure in another, and so would not constitute emotional detachment or a risk of abuse. The UK, however, is seeing an increase in stay-athome fathers and working mothers, and a change in traditional gender roles and socially expected behaviours. In turn, perceived emotional connections with children may be misconstrued. While pertinent to cultural differences in child protective practice, Haley's account is reflective and is not original research into current paramedic practices. Furthermore, Hartley's study was Australian based, and while its findings have validity within UK paramedic practice, no UK-based literature has been identified investigating paramedics' confidence of knowledge of cultural differences affecting child protection. Cultural differences are mentioned in some new textbooks for paramedics (Blaber, 2012), but such mentions are brief and not specifically about child protection. Vydelingum and Colliety (2011) posit that far too many practitioners are still unsure when dealing with cultural, ethnic, and racial aspects of children's care. The extent to which this is the case in UK paramedic practice continues to be unclear and underrepresented within the research literature. Without such information, future practice, policy, and education cannot be informed. Attention now turns to the different types of abuse paramedics may encounter, and their role in identifying and protecting children and young people.

\section{Different types of abuse and neglect}

By exploring the various presentations of abuse and neglect and specifically the extent to which paramedics play a part in recognising it, gaps within the research can be highlighted. This in turn, can help form research questions to achieve the aim of this thesis; that is, to inform future paramedic practice, education, and policy. 


\section{Types of abuse and neglect}

\section{Physical abuse}

Physical abuse may involve hitting, shaking, throwing, poisoning, burning, or scalding, drowning, suffocating, the deliberate induction of illness, or otherwise causing physical harm to a child.

(Department for Children, Schools and Families, 2006. p. 38)

This definition originates from an interagency document called Working Together to Safeguard Children (WTSC). After the 2003 Laming Report into the death of Victoria Climbié, the Children's Act was passed. The Laming Report found that health, police, and social services missed 12 opportunities to prevent her death. WTSC is a collection of statutory guidance documents $(2006,2010,2013,2015)$ which outline the way in which organisations and individuals should work together to safeguard and promote the welfare of children and young people in accordance with the Children Act 2004. WTSC will be explored in more depth within this review. It is worth noting at this stage that all of the WTSC documents mention the role of ambulance services, and thus paramedics, in the role of safeguarding children and young people.

While the presentation of the physical abuse defined here may seem self-explanatory, in clinical practice, it may not be quite as simple. For example, the extent to which physically disciplining a child is abusive is a subjective and often complicated area for practitioners to traverse. Often the trauma cases to which paramedics are called involving children are challenging to manage from a safeguarding perspective. There remain various possible causes of child injury, and many parents can offer seemingly valid explanations. Thus, some health trusts are introducing policies to ask the child what happened before the parents give their version to compare the explanations (depending on age and verbal ability). Similarly, a single incident of trauma which may be explained believably may hide evidence of older injuries, which paramedics may not identify, for example, bruise-over-bruise injuries. It is challenging to mitigate against this difficulty in recognition when patients are discharged at home after treatment and are not taken into $A \& E$, where a more experienced paediatric 
specialist may identify different ages of wounds. There is no readily accessible national data to show how many children are left at home by paramedics after assessment within the UK. Nor is there any readily accessible national data to show how many of these children are subject to child protection plans either at the time of paramedic interaction. This lack of data makes it difficult to fully assess whether paramedics are providing safe and effective child protection without the need for retrospective case analysis, which is often seen only in serious cases and child death reviews. Paramedics may often be the first health professionals to assess a child in their home environment, perhaps uncovering previously unrecognised abuse (Sibson and Brain, 2009 A). Nonetheless, there remains a paucity of research investigating paramedic knowledge, the perception of knowledge, or levels of confidence of child protection within the literature, with no results being returned from the systematic review looking specifically at physical abuse.

There is some emphasis being placed upon physical trauma within the grey literature, as ambulance services produce internal guidelines for injuries of non-mobile babies, for example. However, it is not known to what extent this is available in all ambulance services in the UK or to what extent such guidelines are informing practice. Anecdotally, the collection of paramedic guidelines for the UK most often referred to are the Joint Royal Colleges Ambulance Liaison Committee (JRCALC) UK Ambulance Services Clinical Practice Guidelines (2016). These 2016 guidelines include only six lines of text on the subject of physical abuse in children and there is no further explanation in the 524-page document. The 2017 supplementary JRCALC guidelines have more expansive guidance on various types of abuse, and on the responsibilities of the paramedics, including guidance on new legislation. While this is positive, these guidelines were supplementary to the 2016 guidelines and are available at an extra cost to paramedics, possibly making the purchase and uptake low. While some of the ambiguities may be elaborated upon in local internal (grey) literature, such as ambulance service child protection policies, in paramedic practice, there is a clear lack of emphasis on the possible complexity of physical abuse, despite the documented likelihood of paramedics assessing and treating such abuse in everyday practice. More research is required to fully assess to what extent paramedics know and 
understand their role in recognising physical abuse and exploring from where they get this knowledge.

\section{Domestic violence}

Domestic violence (DV), whether focused directly upon a child or not, is a child protection concern and one which paramedics often encounter (Donnelly et al. 2016), and as such, they need to be aware of the risks, signs, and possible referral pathways. The Crown Prosecution Service (CPS) (2016) describes DV as a serious and pernicious crime which ruins lives, breaks up families, and has a lasting impact. The UK Home Office (2013) defines domestic violence and abuse as:

Any incident or pattern of incidents of controlling, coercive or threatening behaviour, violence, or abuse between those aged 16 or over who are or have been intimate partners or family members regardless of gender or sexuality.

The British Crime Survey (2010-11) shows that nearly 1 million women experience at least one incident of DV each year, and while the effects of physical child abuse are well understood, the effects on children witnessing DV are less well understood. At least 1 in 5 children in the UK have witnessed DV or abuse (Radford et al. 2011). Paramedics need to ensure that they do not focus solely on the physically injured parties of DV, but also focus on the children who may have witnessed and may be at risk of such violence. DV can be experienced by both heterosexual and same-sex partners of either sex, but it is predominantly reported as men against women. Given that women are overwhelmingly the main carers within the home, DV has far-reaching and long-standing repercussions for child witnesses who themselves are likely at times also to be directly abused by the same perpetrator (Hester and Pearson, 1998).

A comprehensive review of the literature by Holt et al. (2008) highlighted that children and adolescents living with DV are at an increased risk of experiencing multiple types of abuse and of developing emotional and behavioural problems, and are more likely to experience adult adversities. These findings make early recognition by any practitioner important, but especially by those who can witness and experience the family and home environment, such 
as paramedics. While this review is now somewhat dated, its implications for practice are arguably still valid. Those children living with DV, but who are perhaps not the sole focus of it, are more likely to commit both non-sexual and sexual offences in later life (Moffitt et al., 2002; Burton et al., 2011). In contrast, those who are identified and supported early go on to gain sound educational attainment, employment security, and economic prosperity (Holt et al., 2008), thus, highlighting how important an issue early identification of childwitnessed DV can be.

Such is the perceived severity of the problem that Canadian law, for example, requires mandatory reporting of DV where a child has only witnessed or heard the assault. Mason et al.'s (2010) study of Canadian paramedics, which used survey data analysed using basic frequency displays and descriptive statistics, suggested that paramedics ${ }^{1}$ lacked sufficient education, training, and knowledge around domestic violence. Similarly, Donnelly et al.'s (2016) study of 403 pre-hospital staff in the USA identified the need for more training, education, and understanding. Donnelly's study suggested that between $32 \%$ to $43 \%$ of the convenience sample reported assumptions and attitudes that indicate beliefs that DV victims themselves are responsible for the abuse they suffer. This perception may have negative implications for the children of DV victims, as they may be seen as abusive by those reporting concerns. Limitations exist in the study design, not least due to a method of convenience sampling at an educational programme, and more research is required from a UK perspective to determine if such negative attitudes exist in ambulance staff and, if so, to what extent. Furthermore, little is known as to what type of knowledge led the respondents to hold such beliefs. Whether they had been taught such knowledge in training, or had come to know it through experiences are valid questions to ask when attempting to inform future education, policy, and practice.

Edlin et al. (2010), in their Australian review of the literature, drew attention to the fact that pre-hospital providers have a unique advantage to identify and report DV over other

\footnotetext{
${ }^{1}$ The Canadian paramedic education and training at the time of this research was in the midst of much change, becoming far more analogous to British and Australian methods.
} 
healthcare professionals. They recognised the paucity of formal research in the area of DV, but asserted that paramedics are frequently the first point of contact in DV cases, as female victims are highly likely to access emergency care via the ambulance services. Furthermore, their review emphasised the link between DV and child abuse and claimed that identification in the pre-hospital setting could lead to early intervention and improved patient outcomes, and that the referral process could lead to the prevention of further episodes of violence to children. However, Edlin et al. argued that to achieve these goals, paramedics need to be competent and confident in identifying victims, recognising environmental clues, and understanding patterns of indicative injury, of which there was little evidence within the literature. What little research that has been identified throughout this review shows that paramedics lack sufficient education, training, and understanding to achieve these positive goals. While these studies were not UK-based, their findings do affirm the need to investigate the training, knowledge, and understanding UK paramedics have about DV and child protection. Furthermore, an investigation into how paramedics acquire this knowledge is important in correcting their incorrect perceptions towards victims of DV, which may affect the children for whom they care.

\section{Youth intimate partner violence}

The violence experienced within domestic relationships can also be experienced in other similar contexts, and paramedics need to be equally aware of it. It might be argued that when professionals who work outside of child protection or social and family services think about violence committed towards a child, they think it to be perpetrated by an abusive adult figure. Abuse, however, is not always committed by adults, and youth intimate partner violence, also known as teen dating violence (TDV), should be considered by paramedics as a possible cause of illness and injury of young people; in turn, this should be reflected in training and education. In 2013, new physical and sexual teen dating violence victimisation questions were added to the United States Youth Risk Behaviour Surveillance Survey (Vagi et al. 2015), which surveyed 9,900 US high school students. Of these students that had dated, $20.9 \%$ of female students $(95 \% \mathrm{Cl}, 19.0 \%-23.0 \%)$ and $10.4 \%$ of male students $(95 \% \mathrm{Cl}$, 9.0\%-11.7\%) had experienced some form of TDV during the 12 months before the survey 
(Youth Risk Behaviour Surveillance United States - Centre for Disease Control and Prevention 2013). Similarly, the 2015 Youth Risk Behaviour Surveillance Survey of 15,624 US high school students showed that among the $68.6 \%$ of students nationwide who had dated during the 12 months before the survey, $9.6 \%$ had been physically hurt on purpose. Among the $69.1 \%$ of students nationwide who had dated during the 12 months before the survey, $10.6 \%$ of students had been forced to do sexual things that they did not want to do ${ }^{2}$, including touching, kissing, and sexual intercourse. While this survey is US-based, the sample size is substantial and indicates a significant child protection concern for teens themselves and for the professionals, such as paramedics, who may be called to assess and treat the injuries or illnesses which result from this violence. The associated range of adverse outcomes of youth intimate or teen dating violence includes mental health problems, depression, suicide, and experiencing DV in adulthood (Cleveland et al., 2003; Collin-Vézina et al., 2006), thus, directly affecting future life prospects.

Little is known about the prevalence of UK paramedic involvement in TDV cases; however, given the likelihood of their involvement in the assessment and treatment of victims within the community, it is important to understand their views and perceptions. This understanding is especially important if their views and perceptions mirror those of previous studies of DV. Barter et al.'s (2009) research into UK youth intimate partner violence concluded that high levels of violence in young people's relationships (as found within this research) testifies to the need to develop and initiate professional responses aimed at protecting children's and young people's welfare. Paramedics can be part of that professional response, although the literature is scarce surrounding the roles $A \& E$ and paramedics play in the recognition of such intimate partner violence. Applying clear roles and responsibilities to paramedics regarding TDV is highly difficult given that very little is known about such cases, in contrast to the substantial body of UK evidence related to adult experiences of intimate partner violence (Barter et al. 2009). Temple et al. (2013) state that

\footnotetext{
${ }^{2}$ The prevalence of dating or going out with someone during the 12 months before the survey varies slightly for physical dating violence and sexual dating violence because of differences in the number of usable responses to each question.
} 
youths exposed to such violence should be identified through shelters, emergency rooms, and other settings. Arguably, the community is such a setting, and teens are often taken to A\&E by paramedics from settings such as shelters and safe houses. Indeed, paramedics play a key role in screening and identifying youth victims of DV and TDV, but nonetheless, there is a paucity of research investigating whether paramedics consider this to be part of their role, and if so, on what knowledge sources they draw to support this role in practice.

\section{Emotional abuse}

Emotional abuse is also known as psychological abuse or chronic verbal aggression. While most people appear to know what physical abuse is, it might be argued that emotional abuse is more difficult to define and recognise. WTSC (2010 p. 38) defined it as

The persistent emotional maltreatment of a child such as to cause severe and persistent adverse effects on the child's emotional development; and may involve conveying to children that they are worthless or unloved, inadequate, or valued only as far as they meet the needs of another person.

The presentation of emotional abuse or neglect may seem self-explanatory from the above definition; however, in practice, it may be more difficult to recognise. Emotional abuse can be an overlapping element of all other types of abuse as well as a single behaviour in isolation. This conflation makes estimating the prevalence of child emotional abuse challenging, and estimating the service need, the level of investigations, and the number of referrals is also challenging (Sibson and Brain, 2009 A). Knowing how many cases of emotional abuse paramedics encounter is also difficult, as no literature specifically refers to paramedic involvement in emotional abuse, but rather in various types of abuse which may have undertones of emotional abuse.

It is estimated that 1 in 14 children have experienced emotional neglect by a parent or guardian (Radford et al. 2011). In 2014, the NSPCC reported to the British Broadcasting Corporation $(\mathrm{BBC})$ that the number of cases of emotional abuse deemed severe enough to be reported immediately to the police and child protective services was increasing, with a 
$47 \%$ increase in referral rate from 2013 to $2014^{3}$ (BBC, 2014). The area of emotional neglect has recently been subject to public scrutiny in the wake of a recent campaign by UK charity 'Action for Children'. In 2014, it was announced in the Queen's speech that existing laws covering child abuse and neglect would be updated to criminalise emotional neglect and abuse. Emotional neglect comprises exposing children to domestic abuse or constantly humiliating and belittling a child, and it can be as damaging and as long-lasting as physical harm. It is difficult, however, to understand at what point a parent's inability to show affection becomes emotionally neglectful and how this may be identified and taken forward in clinical practice. Furthermore, the mental health issues suffered by the parent/carer can also hamper their ability to portray and share meaningful emotion, and there needs to be research into how this is safeguarded against.

Paramedics must be able to recognise not only the multiplicity of factors dictating, precipitating, and resulting from emotional neglect, but also have a role in reporting it and referring concerns. The extent to which this is currently happening is difficult to assess without sufficient data. Thus, more research is required to assess paramedics' views, perceptions, and levels of competence in this area of practice, and from where they gain their knowledge. There is little literature focusing upon how issues of emotional neglect are dealt with in paramedic practice, education, or training. There is some passing theoretical reference to emotional neglect in the national paramedic guidelines from 2013 and 2016, but more are provided within the 2017 supplementary guidance released from JRCALC (JRCALC, 2013, 2016, 2017). These supplementary guidelines updated readers on various aspects of emotional abuse, but still at only a foundation level, consisting of only half a page. There is a section within Blaber's (2012) textbook Foundations for Paramedic Practice: A Theoretical Perspective, but little regarding methodological research. This is despite emotional neglect interlinking with and overlapping many other areas of child abuse and

\footnotetext{
${ }^{3}$ Helpline staff have assisted over 8,000 people who have contacted the NSPCC about emotional neglect and abuse in the period 2013/14 - 5,354 of these cases were so serious they were referred to local authorities for further action compared to 3,629 in the previous year $(2012 / 13)$.
} 
neglect. Without this research, future paramedic education, policy, and practice cannot be informed.

\section{Parental/carer mental health issues}

Mental health problems are among the most common of health conditions in the UK and directly affect about a quarter of the UK population (Halliwell et al., 2007). WHO (2003) states that mental health problems affect the whole of society, and are most common amongst the poor, homeless, elderly, unemployed, poorly educated, victims of violence, and children and young people. An estimated 800,000 people worldwide commit suicide every year (WHO 2011). Common mental health conditions include depression, anxiety, phobias, schizophrenia, bipolar, post-traumatic stress, and seasonal affective disorders, while common behaviours include self-harm and suicidal thoughts as well as panic attacks. These behaviours and symptoms can sometimes inhibit a parent's ability to engage with their child emotionally and can manifest in them appearing distant, uninterested, unloving, and unstable.

The effects of poor parental/ carer mental health on children and young people have become more prominent in recent years, and this issue is now well documented and supported by increasing amounts of mixed methodology research. Letourneau et al. (2013) undertook a longitudinal study of relationships between maternal symptoms of depression and children's cognitive behavioural development. The outcomes concluded that children of depressed mothers are at an increased risk of having a low receptive vocabulary and of displaying inattention and physical aggression at a very early age. These authors, in turn, call for clinicians to remain vigilant for signs of post-partum and maternal depression (although single father families should not be discounted), and to approach these disorders holistically. The Children's and Young People's Health Outcomes Forum (CYPHOF) (2012) emphasises how important positive parenting and a 'good start' in life are to good mental health and wellbeing, as well as lifelong resilience to adversity. This is worth noting, given that half of all lifetime mental illnesses (excluding dementia) have already developed by the age of 14 (Kessler et al., 2005). Paramedics need to be able to identify such disorders and 
consider any warning signs or risk factors when assessing and treating parents and caregivers. This assertion is not unreasonable considering that the majority of cases that UK paramedics encounter are psychosocial in origin (DoH, 2005) as opposed to life-threatening emergencies.

The regulatory body presiding over paramedics within the UK states that practitioners should be able to manage those with psychosocial concerns appropriately, and should have an in-depth knowledge of the psychosocial factors that influence an individual's health and illness (Health Care Professions Council (HCPC), 2014). Nonetheless, there appears to be a gap in the literature in relation to paramedics' efficacy in such areas. Mental health patients comprise a growing proportion of the paramedics' workload (O'Sullivan 2014), but while paramedics' assessment of patients and families is essential in determining the appropriate treatment and referral, their education, training and assessment techniques are lacking (Robert and Henderson, 2009). Multiple authors have suggested that psychosocial care is considered less of a priority than other forms of care because the focus is still predominantly on physical illness or injuries (Roberts and Henderson, 2009). This is arguably prevalent within paramedic practice (Jones et al., 2010: Brady, 2012) despite paramedics being the first point of contact for an increasing number of mental health patients and irrespective of the effects these conditions can have on children and young people. More research is required to determine if paramedics understand the connection between parental mental ill-health and child protection, and if so, to what extent they think that recognition and referral is part of their role. The normal social narrative is that children are reliant upon their parents for support, care, and nurture to thrive; in cases of both mental and physical parental ill-health, however, this can be reversed. Children and young people often become carers themselves.

\section{Youth carers}

There are thought to be around 700,000 young carers in the UK (Carers Trust, 2017), although it is difficult to truly determine the figures given the often-unrecognised roles young carers play and the lack of input they have from formal services. Cooklin (2006) 
identifies multiple risks to young carers of parents with mental health problems, such as feelings of vulnerability, a sense of being ignored, the pressure to be 'good', false maturity, and the fear of family breakdown. Interventions such as young carers support groups, afterschool activity clubs (not involving parents), and the presence of an alternative caring adult have been shown to be support mechanisms. These mechanisms can assist in the alleviation of undue stress, anxiety, poor self-esteem, lack of social interaction, and poor educational attainment to which young carers are susceptible (Cooklin, 2006). These interventions may be required more specifically in and after times of acute crisis, which paramedics may encounter within their practice. Manning and Gregoire (2006) emphasise the importance of early identification, prevention, and intervention for young carers, to limit the increased risk of them developing psychiatric disorders in childhood, adolescence, and later adult life. To do this effectively, however, all health professionals who encounter young carers need to understand the challenges they face and the risk factors of crisis.

Paramedics are likely to be involved in crises (medical and psychological) as those concerned will often call 999. As a result, paramedics have a responsibility to recognise the risk factors and to refer vulnerable children and young people to support agencies. However, there is a lack of research investigating paramedics' knowledge of young carers and their perceived role in the protection of the children and young people who care for their family members. Without this research, future education, practice, and policy cannot be informed, and the extent to which improvement strategies are required cannot be fully assessed. This type of child protection concern is not as well-known as other types, such as sexual and physical abuse, which will be explored next, but it is equally important, given the risk of the resultant poor adult outcomes.

\section{Sexual abuse}

Sexual abuse is defined as follows:

Abuse that involves forcing or enticing a child or young person to take part in penetrative and non-penetrative sexual activities, or in the production of sexual images; not necessarily involving a high level of violence, whether or not the child is aware of what is happening. Women can also commit acts of sexual abuse, as can other children.

(DCSF, WTSC. 2010 p. 38) 
Those who have been sexually abused are more likely to have poorer psychological wellbeing, greater rates of teenage pregnancy, a higher likelihood of multiple victimizations, and a lower socioeconomic status in adulthood than non-victims (Seto et al., 2015). Understanding the role paramedics play in identifying and preventing this abuse is important in mitigating these poor outcomes. Stoltenborgh et al. (2011) conducted an extensive meta-analysis on the prevalence of child sexual abuse across the world. Based on 331 independent samples and almost 10 million individuals, Stoltenborgh et al. found an overall prevalence rate of $13 \%$, with the rate for girls being more than twice that for boys (18\% vs $8 \%$, respectively). Due to the nature of all sexual abuse and the recognised lack of reporting and subsequent recording, the exact numbers remain difficult to estimate (Horvath et al., 2014). It is thought, however, that up to one-third of child sexual abuse is perpetrated by family members and is considered a widespread social and health problem (Seto et al., 2015).

The 2015 the Children's Commissioner for England published a report titled Protecting Children from Harm: A Critical Assessment of Child Sexual Abuse in the Family Network in England and Priorities for Action. This rapid evidence assessment (REA) on interfamilial child sexual abuse identified a total of 57,226 relevant articles and analysed a total of 296 . Data were also gathered from every police force in England on all victims of child sexual abuse over a two-year period, April 2012 - March 2014, as well as data held by the Department for Education on statutory child protection processes. An open "Call for Evidence" enabled all organisations working on this issue to provide case studies and other forms of evidence to the Inquiry. Alongside this, a questionnaire was distributed to adult survivors of child sexual abuse in the family, to which 756 responded (the largest sample to date). The statistical model Multiple Systems Estimation (Petersen, 1896) was used to estimate the number of unknown victims of child sexual abuse (a methodology which has been well established for finding unknown victims of various crimes). This methodology suggests that approximately only 1 in 8 victims of sexual abuse come to the attention of statutory agencies. Although the limitations of the statistical model are recognised, given such a low detection and disclosure rate, the roles of professionals have been subject to a great emphasis within the 
recommendations of this report, although no specific mention of ambulance services was made.

Paramedics are often in an adventitious position within the community to identify and monitor children and young people through the access they gain in emergency situations, something not often afforded to other professionals (Brady 2012). Given that a significant proportion of sexual abuse is thought to be perpetrated by family members, what paramedics do with this access is highly important. However, little is known about paramedics' perceptions of their role in identifying and preventing sexual abuse, and specifically, their perceptions of their access to people's homes. This review has identified a scarcity of literature investigating paramedic confidence levels, knowledge, and perceptions of sexual abuse. Findings from the literature that is available can be extrapolated to inform paramedic practice to a certain extent, but without specific research investigating paramedic involvement, confidence, and knowledge, then future practice, policy, and education cannot be informed and improved. Given the access paramedics have to the home environment and given that families may not view them in the same light as the police, health visitors, or social services, they are in a prime position to identify problems in the first instance (Kirtley, 2013), and their roles must be investigated.

While this access is important, it must be remembered that sexual abuse is not always interfamilial, and paramedics need to be prepared (educationally) to recognise abuse that happens away from the home environment. Paramedics also encounter children and young people outside of the home environment at youth clubs, sports centres, schools, skate parks, and just in the streets, and they are often called at times of crisis, injury, or concern. It is important to recognise this interaction, especially if it takes place during school time or with a young person who has run away, given that these can be signs of concerns. When children and young people are away from home, they may feel safer to disclose interfamilial abuse, or in the presence of paramedics, they may feel safer to disclose information about non-interfamilial abuse which is possible occurring at the location at which they find 
themselves. Paramedics may be able to identify signs of sexual abuse at the hands of group leaders, religious officials, and sports coaches, when they are called to these alternative environments. Little is known, however, about paramedics' confidence or knowledge of such interactions in relation to child protection.

This kind of non-interfamilial sexual abuse has recently been forced to the forefront of public policy, with multiple investigations into high-profile institutional and individual cases. Jimmy Saville, for example, was alleged to have been able to hide behind a facade of celebrity for many years. Following a barrage of posthumous allegations of widespread institutional sexual abuse, a public and criminal enquiry was launched. Operation Yewtree soon had more than 400 lines of inquiry involving 300 victims of whom all bar two were women. Allegations spanned three decades in multiple locations and organisations. This investigation concluded with a joint report by the NSPCC and the Metropolitan police titled Giving Victims a Voice (GVV) (Gray and Watts, 2013). GVV (2013) highlighted how Savile is said to have committed most of the alleged abuse during a time when social attitudes and the workings of the criminal justice system were completely different from today. It highlighted the systemic failings by many individuals and organisations to safeguard children and identified a culture whereby most victims felt that they would not be believed, given the abuser's social standing. The case of Saville and the subsequent GVV (2013) report empowered many victims of non-recent sexual abuse (at the hands of individuals, Roman Catholic and Anglican organisations, youth football clubs, child protective services, and care homes) to come forward to the authorities, whom they now believed would take them seriously. These revelations forced the UK authorities to re-evaluate the training and education around the identification and reporting of sexual abuse, and caused organisations across the public, voluntary, and private sectors to re-evaluate their approaches to child protection.

Despite these changes, there is still a paucity in the research literature pertaining to UK ambulance services and the roles of their paramedics in identifying sexual abuse of all kinds. While the literature cited within this section informs paramedic practice to a certain extent, more specific research is needed from a paramedic perspective to investigate how 
paramedics perceive their role in child sexual abuse, and crucially, to investigate if lessons learned from the wider UK context have been applied to paramedic practice. There is also little research investigating paramedic education, understanding, and confidence in recognising sexual abuse, all of which is essential given their position within the community. As stated previously, sexual abuse is often a term used for interfamilial abuse towards children; however, sexual abuse can also take the form of exploitation, as will be explored in the next section.

\section{Child sexual exploitation}

Child Sexual Exploitation (CSE) is described by the NSPCC (2017) as a type of sexual abuse in which children in exploitative situations and relationships receive something, such as gifts, money, or affection, as a result of performing sexual activities or others performing sexual activities on them. These children or young people may be tricked into believing they are in a loving, consensual relationship, and might be invited to parties and given drugs and alcohol as well as being groomed and exploited online. Kirtley (2013) expands upon this description and states that such behaviour often involves a power imbalance whereby the perpetrator has power over a victim due to their age, gender, intellect, physical strength and economic resources.

The awareness surrounding CSE has increased in recent years and stems from several highprofile cases in which reports of widespread CSE were seemingly ignored. In response to this growing awareness, several official enquiries (e.g., Jay 2013) and serious case reviews have recently been undertaken. Gadda and Taylor (2015) explain that these reports have highlighted the failure of agencies to work together and share information. Specifically, health services (amongst others) have failed to recognise the early signs of CSE, and health staff lack any understanding of child protection procedures and information sharing policies. Jay (2013), in her report titled Independent Inquiry into Child Sexual Exploitation in Rotherham, highlighted that multiple professionals failed to detect and report the CSE affecting many hundreds of children during this time. However, while her report mentions nurses, police, and accident and emergency departments, there is no mention of ambulance 
services or paramedics. Rotherham and the surrounding areas sit within the Yorkshire Ambulance Service (YAS) geographical area. This researcher contacted YAS to inquire about their involvement in Jay's (2013) report. They confirmed that they were not contacted and, indeed, did not participate in any way in this report. In addition, due to time constraints, they were also unable to provide information regarding child protection referrals made by YAS paramedics between 1997 and 2013 for suspected CSE. They concluded that due to their lack of involvement in the report, no shortcomings in YAS practice were highlighted. Interestingly, YAS do not conclude that there were no shortcomings, but only that this report highlighted no shortcomings. No internal investigation took place with YAS to investigate the findings from this report. Drawing the conclusion that a lack of involvement in a report equates to a lack of shortcomings would not be valid, especially given the examples within the review of reports, investigations, and research that fail to consider the involvement of paramedics. Given the plethora of problems that arise from CSE, it seems likely that paramedics at some point may have encountered victims of CSE, especially if they had been taken to emergency departments.

A 2013 inquiry conducted by the Children's Commissioner into gang and group sexual exploitation concluded that at least 16,500 children were identified as being at risk of CSE during one year of this two-year study. Furthermore, 2,409 children were confirmed as victims of sexual exploitation in gangs and groups during the 14-month period from August 2010 to October 2011. Examples of CSE in this report include cases of children being raped, abducted, trafficked, beaten, and threatened after being drawn into a web of sexual violence by promises of love. The report revealed children suffering in silence for years as they are casually and routinely raped as they come out of school, as they walk to the shops, and as they play in their local park, by perpetrators who range in age from as young as fourteen to the elderly and who are from all ethnic groups. These figures are suspected to be far greater, in part due to agencies' failures to recognise, report, and act upon findings, and this further supports the assertion that paramedics and other community practitioners are highly likely to interact with victims of CSE or at least those who are at risk. The preceding 2012 interim report and call for evidence received 115 submissions of evidence 
and led to interviews with 68 health and social care professionals and 23 sexually exploited children. There was, however, a notable absence of submissions by and interviews with paramedics.

This report identified the high risk of CSE victims having drug and alcohol problems, selfharm tendencies, and poor mental health, which often resulted in admission to accident and emergency, yet the report failed to refer to those who are likely to assess these victims before their admission, or who are responsible for their admission. Furthermore, supporting information of this 2012 interim report states, 'In an emergency, please dial 999', thus highlighting the involvement of emergency services, but not fully recognising their possible role. The omission of paramedics from such a report suggests a lack of understanding about their advantageous position within the community and the role they could play in identifying and treating CSE victims. Paramedics work alongside many of the professional groups mentioned within these reports, and while the authors cannot mention every professional group, the question arises of whether authors and researchers are underestimating or are unaware of the role paramedics play within the community ${ }^{4}$. The 2017 JRCALC guidelines do have a small section on CSE and cover the various forms: peer on peer, boyfriend model, party model, and exploitation through befriending. Nonetheless, given the possibility of paramedics encountering victims or those at risk of victimisation, this half-page inclusion does not appear to be sufficient to form national standardised guidance.

The role paramedics play in recognising and reporting CSE within the community was recognised by Kirtley (2013) in his document Report of a Grass Roots Survey of Health Professionals with Regard to their Experiences in Dealing with Child Sexual Exploitation. Kirtley focused on many different disciplines, including school nurses, A \& E departments, sexual health services (SHS), midwives, and ambulance services (paramedics). Kirtley's report recognised that paramedics often have direct entry into homes which other professionals are either denied or lack. Furthermore, it was recognised that paramedics may

\footnotetext{
${ }^{4}$ This was put to Rotherham Metropolitan Borough Council, who stated that as an independent author undertook the inquiry, they were unable to respond. The author, Professor Jay, was contacted, but did not reply, even after multiple attempts.
} 
receive information about irregularities in the history of a case because call centres often get different stories from those given to the paramedics who arrive on the scene. With 999 calls being recorded, it is also possible that evidence may be inadvertently recorded, such as shouting parents or crying children. Despite this position, however, there appear to be poor feedback mechanisms, deficient CSE education, a lack of information sharing, and a wide disregard of the role that paramedics play in CSE treatment by other disciplines, all of which hamper the effective identification and treatment of CSE by paramedics (Kirtley, 2013). Kirtley's findings are unique in that they offer an insight into an otherwise unexplored area of paramedic practice. There remains a lack of further research investigating the role of paramedics in CSE identification, and as such, other disciplines may remain unaware of the possibilities of this role. Without research and evaluation, it is not known if improvement strategies or educational interventions are needed, and to what extent. Without this research, victims may not be identified as early as they possibly could be, and given that paramedics are best able to help in the first instance (Kirtley, 2013), paramedic-focused research is needed.

\section{Female Genital Mutilation}

Female genital mutilation (FGM) is also known as female genital cutting, sewing, and female circumcision. This procedure is predominantly practised in 28 countries worldwide on females between infancy and the age of 15 (WHO, 2016), although it can happen in any country and can be undertaken by anyone of any religious affiliation. FGM is believed to be associated with cultural ideals of femininity, modesty, beauty, and cleanliness, and the removal of all body parts considered male or unclean (WHO, 2016). These procedures are often carried out by untrained community leaders, and all are now considered a breach of a woman's human rights by the United Nations (UN).

Peate (2014) states that the paramedics' position as front-line members of staff allows them to identify and protect those at risk of FGM. Furthermore, paramedics are well placed to identify victims of FGM through the identification of common consequences, such as vaginal bleeding, overwhelming infection, and abdominal pain. This line of thinking is congruent 
with that of the UK House of Commons Home Affairs Committee (2014), whose report on FGM highlights the vital role health care professionals play in breaking the generational cycle of FGM. This report identified the lack of a consistent approach to identifying girls at risk and introduced new community pilot schemes, such as automatic referral for the daughters or unborn children of women from FGM-practising countries, and it mentioned specific educational packages for community professionals, such as children's social care teams, the police, health visitors, and GPs. Despite the similarities paramedics have regarding access, responsibility, and the likelihood of interaction with these vulnerable females, they are not mentioned specifically in this report. Without recognition from central government, it might be argued that other professions might not fully understand the role paramedics play in identifying FGM, which may in turn, limit training and funding, and the impetus for research. Peate (2014) was writing in an editorial piece in a paramedic specific journal, which might limit its readership to paramedics only, thus compounding this possible issue. No research literature has been identified as part of this review which investigates the role paramedics play in the identification of FGM or their own perception of this role. This lack of literature and apparent research impetus is in direct contrast to the focus placed upon health care professionals (HCPs) in the recently approved Serious Crime Act (2015).

The introduction of the Serious Crime Act (2015) placed a legal obligation on HCPs to report signs, symptoms, and suspicions of FGM to the police and allowed them to breach what would normally be considered patient confidentiality. The College of Paramedics (2015) stated that it is important for professionals on their register in England and Wales to be aware of the new statutory duty, as failure to comply would be dealt with via the HCPC 'fitness to practise' process. This is because the legislation does not provide for a breach of the duty to be addressed by criminal sanctions. Interestingly, however, there is still currently no legal obligation for professionals to report suspected FGM in Scotland and Northern Ireland. Given the legal obligation for paramedics to report signs, symptoms, and suspicions of FGM, as well as the warning from the registering body of UK paramedics, the HCPC, it would be reasonable to expect there to be increasing amounts of literature pertaining to paramedic training and awareness of FGM. However, this review found few results. JRCALC 
(2017) included three short paragraphs on the mandatory reporting of FGM, making clear to paramedic readers their responsibilities; however, this guidance came some three years after the legislative requirements, and only in supplementary guidance to the 2016 JRCALC publication, as mentioned earlier.

Little is known about the education paramedics currently receive regarding FGM, although limited and sporadic access to the grey literature may mean that internal ambulance service updates have not been included in this review. Given that FGM causes significant short- and long-term physical and psychological damage, paramedics need to consider the possibility when attending all female children and young people with genital injuries, walking problems, urinary tract infections, and other gynaecological or maternity complications. The literature in relation to paramedicine and FGM is scant, and little is known about paramedics' education, policy, practice, and their own perception of their involvement in FGM identification. This lack of literature and research is not confined to FGM, however, as child neglect is explored next.

\section{Neglect}

The awareness surrounding neglect has increased recently, in part due to new laws surrounding emotional neglect within the UK. Neglect is the persistent failure to meet a child's basic physical and psychological needs and is likely to result in the serious impairment of the child's health or development. Neglect may also occur during pregnancy, or once a child is born, and can involve lack of food, protection, emotional support, and appropriate medical treatment (DCSF WTSC, 2010 p. 39).

Parental or carer neglect can take many forms, too many for this review of the literature to cover. Cases of neglect often involve individual, family, and socioeconomic circumstances ranging from physical, educational, emotional/psychological, and medical neglect. See

Figure 2. 
Figure 2 - Types of Neglect (Child Welfare Information Gateway 2016)

\begin{tabular}{l|l} 
Types of Neglect & Description of Neglect \\
\hline Physical & $\begin{array}{l}\text { Involves parent or carer not providing necessities, such as food, } \\
\text { shelter, and clothing, thus, affecting physical growth, development, } \\
\text { and wellbeing. This can also involve abandonment, rejection, and } \\
\text { inadequate supervision. }\end{array}$ \\
Educational & $\begin{array}{l}\text { Involves parent or carer failing to enrol a child of compulsory school } \\
\text { age in education, or to provide homeschooling, thus allowing chronic } \\
\text { truancy and educational failure. }\end{array}$ \\
Emotional / & $\begin{array}{l}\text { Involves parent or carer constantly belittling the child, withholding } \\
\text { affection, allowing the child to use drugs or alcohol, and allowing the } \\
\text { child to witness domestic violence. }\end{array}$ \\
Medical & $\begin{array}{l}\text { Involves parent or carer failing to provide appropriate and necessary } \\
\text { health care for a child, thus placing the child at risk of being seriously } \\
\text { disabled or disfigured or dying. }\end{array}$ \\
\hline
\end{tabular}

Given the nature of a child's physical and cognitive immaturity, they are reliant on others for the necessities to survive, grow, and develop. Neglect is often solely thought to involve the malicious failure of a parent or carer to provide these necessities. However, neglect can occur for several reasons. In addition to malicious neglect, for example, neglect can occur due to a misunderstanding of a child's needs (intellectual limitations), misunderstanding of developmental landmarks (toilet training), unrealistic expectations of a child's abilities, parental mental ill-health, and neglect due to financial adversity. Paramedics have a key role in recognising neglect or failure to thrive when assessing and treating children and families (Brady, 2012, NSPCC), as they have access to home environments and may detect the need for support or referral when called to deal with many other medical needs. This review has found literature that recognises the role paramedics play in identifying neglect, but no research investigating their role and their perceptions of this role. Much of the literature identified is based on reviews and is theoretical in nature, but is lacking in up-to-date supporting evidence, which is necessary to inform future paramedic education fully, as well as policy and practice. 
While many cases of neglect feature an omission or withholding of something required to thrive, neglect can also be self-inflicted - predominantly with young people and young adults. Self-neglect should be considered by paramedics, especially if they encounter children and young people out of school during term time or in inappropriate settings. The term 'self-neglect' is well known in geriatric medicine, but is far less researched as a speciality in child and young person health and social care. Self-neglect is often synonymised with self-harm, inappropriate sexualised behaviour, and self-injurious behaviour. Selfneglectful or high-risk behaviours are common amongst those who have been abused, and victims are at risk of experiencing poor physical health, mental health disorders, educational failure, unemployment, substance addiction, and homelessness (Lazenbatt, 2010; Gilvarry et al., 2012).

The identification and treatment of this behaviour in adolescents is two-fold for paramedics in that they often have to treat the directly harmful behaviour (such as overdoses or selfmutilation), while also considering that such behaviour can be a symptom of abuse and neglect. Self-neglect can come in the form of physical self-abuse, truancy, and homelessness, and highlights how multifaceted child and adolescent practice is. It is recognised that paramedics often encounter those who are homeless and those who have self-harmed (Zakrison et al., 2004; Rees et al., 2015) and so have an important role in the identification, treatment, and referral process. Despite this recognition, the literature included within this review was theory based or consisted of generalised literature reviews. No research was identified that specifically investigating paramedic involvement in paediatric self-neglect. This arguably contributes to an ill-informed practice and policy base for UK paramedicine. This lack of research is also evident in areas such as educational neglect.

The Education Act (1996) sets out the legal right and necessity for children of compulsory school age ${ }^{5}$ to receive efficient full-time education that is suitable to their aptitude and

\footnotetext{
${ }^{5} \mathrm{~A}$ person begins to be of compulsory school age when s/he attains the age of five.
} 
special needs. It has been identified that children missing education (CME) are at significant risk of underachieving and becoming NEET (Not in Education, Employment, or Training) later in life (DOE 2013 CME p. 4). The National Children's Bureau (NCB) (2014) identified a potential 14,800 children being identified as CME in 2014 England alone. More recently, the BBC completed a Freedom of Information request, which identified that 33,262 school-aged children were recorded as missing from education in the academic year ending July 2015 (Talwar, 2016). These more recent figures were based upon the narrow statutory definition of $\mathrm{CME}$, and so actual figures may be significantly higher (Ryder et al., 2017). Currently, the UK government does not collect national-level data, meaning the scale of the problem is relatively unknown.

CME are likely to be victims of abuse and neglect and are at risk of physical, emotional, and psychological harm, particularly if their whereabouts are unknown (National Children's Bureau (NCB), 2014). Recent cases involving CSE and forced marriage (which became illegal in the UK in 2014 under the Anti-Social Behaviour, Crime and Policing Act 2014) have included children who were missing education, thus highlighting the importance of early recognition. Paramedics are often able to recognise CME through their work within the community. Suspicions should be raised if paramedics are called to houses where children are at home during school times or called to the community to treat those who should be at school. The NCB (2014) also cites certain groups of children with increased vulnerability, including children of gypsy, Roma, and traveller families; children of the armed forces; missing children; and runaways; as well as children and young people supervised by the youth justice system. Paramedics could inquire about school attendance as part of their social history gathering, and could then refer any concerns they might have.

\footnotetext{
A person ceases to be of compulsory school age at the end of the day which is the school leaving date for any calendar year-

(a) if s/he attains the age of 16 after that day but before the beginning of the school year next following,

(b) if $s /$ he attains that age on that day, or

(c) (unless paragraph (a) applies) if that day is the school leaving date next following his attaining that age.
} 
HCPs have a duty 6 to identify CME, especially because of the long-term personal and societal effects being NEET can have. Truancy itself is arguably a form of educational selfneglect and should be treated as seriously as parents failing to provide or ensure education for their children. Paramedics may encounter children and young people daily during school days, where the question ought to be asked: Has the child, young person, parent, or school adequately explained the absence, and if not, should a referral be made? Recent statutory guidance from the Department for Education sets out the need for local authorities to regularly raise awareness of their policies and procedures and notification routes with those working with children and families, such as GPs, other health professionals, clinical commission groups, and the police and other emergency services (DOE 2016). One questions, however, if paramedics routinely ask questions surrounding school attendance and if they fully understand the importance of CME recognition, especially in cases such as youth homelessness.

Little is known, however, about how paramedics perceive their role in this area of child protection and how they understand their statutory responsibilities. The literature in this area appears to be generic and in textbook and internal policy form with no identified research investigating paramedic knowledge, experience, or understanding specifically. Furthermore, in university or ambulance service education, little is also known about how much emphasis is placed upon neglect of any kind. Neglect can be identified by paramedics through everyday encounters with children and families within the community, and there is a need to understand paramedics' perceptions and confidence levels of neglect so that future practice, education, and policy might be informed and improvement strategies or educational interventions can be validated and or improved if needed.

\footnotetext{
${ }^{6}$ Under the Education Act (1966), parents have a duty to ensure that their children of compulsory school age are receiving efficient full-time education, and schools and academies have safeguarding duties in respect of their pupils, and as part of this, should investigate any unexplained absences.
} 


\section{Self-Harm}

Self-harm describes a range of behaviours including but not limited to cutting, scalding, hair pulling, breaking bones, overdoses, drinking to excess, stabbing, and strangling. Self-harm is a child protection concern and should not focus solely on children and young people, but should also include the parents or carers of these children and young people should they self-harm. The Mental Health Foundation (2016) explains that self-harmful behaviour is often hidden and secretive and can go on for a long time without being discovered. It goes on to state most people who self-harm are aged between 11 and 25, with harmful behaviour often continuing into adulthood. The Health Behaviour in School-Aged Children (HBSC) report (Brooks et al., 2015) asked 1,608 15-year-olds about self-harm. It revealed that $22 \%$ of these 15 -year-olds had hurt themselves in the previous year. In this report, the sample comprised 6,181 eligible pupils, and 5,679 of these pupils returned at least partially completed questionnaires, resulting in a response rate of $92 \%$ at the pupil level. The study involved young people in England aged 11, 13, and 15, and, disconcertingly, showed a near tripling in the number of children who reported self-injurious behaviour in comparison with a similar report in 2002. Only 15-year-olds, however, were asked about self-harm (for an unknown reason), and so it might be argued that these figures under-represent the incidence rates in school-aged children.

The reasons behind self-injurious behaviour are multifaceted. The Royal College of Psychiatrists (2012) explains that, unfortunately, some young people use self-harm as a way of trying to deal with very difficult feelings that build up inside. Furthermore, it states that those who self-harm do so to feel more in control, relieve tension, punish themselves, and to feel more alive and connected with reality. Some young people who self-harm do, however, go on to commit suicide, and suicidality should always be considered. Causes behind negative feelings may result from depression, anger, guilt, and helplessness and can often be a coping mechanism for issues such as abuse, trauma, and neglect.

It is important for paramedics to view self-harm in two ways. The physical wounds themselves require medical assessment and treatment, but the underlying causes also need 
assessing and referral for possible treatment. O'Sullivan (2014) states that paramedics may be the first point of contact for increasing numbers of mental health patients; however, they tend to focus more on repairing physical injuries, with less attention being given to the patient's experience of their self-harm. The purpose of O'Sullivan's article was to stimulate discussions and reflections among paramedics, and it undertook no original research, although it did allude to children and young people who self-harm. Rees et al. (2014) conducted a systematic review of the quantitative literature investigating the perceptions of paramedics and emergency care workers of those who self-harm. Their study included only 16 papers and highlighted that while some research has been undertaken into the attitudes of emergency nurses and doctors, very little has been undertaken by ambulance service staff, and none has been carried out into modern professional paramedic care. Rees et al.'s (2014) study highlights examples of literature of a varying quality and concludes that there is a need for more research, education, and policies to guide the care of these patients. Only one of the results referred directly to ambulance staff; however, as this was dated 1978, there are obvious limitations regarding its applicability to modern practice. The results from which Rees et al. draw their conclusions were predominantly adult based, and only one study appeared to look specifically at young people; although not children.

This study was followed up by Rees et al. (2015) with a constructivist meta-synthesis of the qualitative literature. Of the 12 papers which were finally reviewed, none investigated paramedic care for self-harm, and thus this study relied on constructivist interpretations and translations of paramedic studies from a wide range of practice contexts. This paper identified no results specifically investigating self-harm by children and young people. Nevertheless, the study highlighted various themes: (a) frustration, futility, and legitimacy of care; (b) first contact in the pre-hospital environment - talking, immediate and lasting implications of the moral agent; (c) decision-making in self-harm - balancing legislation, risk and autonomy; and (d) paramedics' perceptions- harnessing professionalism and taking advantage of opportunities to contribute to the care of self-harm. When compared alongside the findings from Rees et al.'s (2014) systematic review of the quantitative data, the (a) frustration, futility, and legitimacy of care themes may indicate more reasons why 
self-harm patients felt they have had negative experiences with ambulance staff. The (b) talking, immediate and lasting implications of the moral agent metaphor highlighted how the sporadic environment of emergency care posed a nontherapeutic environment for many self-harm patients which, in turn, may lend itself to a poor experience. Although interesting and possibly applicable to children's and young people's practice, the findings are limited. One questions if the negative views of those who self-harm are similarly held of children and young people, and if so, what effect this has on effective referral.

The Mental Health Foundation's (2006) national inquiry into self-harm among young people identified that several of them had experienced negative and sometimes hostile reactions from ambulance staff. However, while some patients may have been told that they were wasting time and resources, other patients had found paramedics to be supportive, compassionate, and understanding of their needs. The reference to hostile and negative reactions is backed up by the range of evidence and presentations heard by the Inquiry from the ambulance staff themselves, which illustrated how self-harm seems to be a difficult and challenging matter for them.

Ambulance staff need to be able to react and respond appropriately to a young person whose need for urgent medical attention is often, in effect, the first time that their selfharm has come to light (Mental Health Foundation, 2006). The only specific national guidance that ambulance staff currently have is a small two-paragraph section in the JRCALC (2016) Clinical Practice Guidelines introducing information around self-harm and patient consent. Little is known about how paramedics perceive their role towards children and young people who self-harm, and less is known about their confidence levels regarding this issue and any training that underpins this. Paramedics are uniquely placed to positively influence the dominant narrative of those individuals who present with self-harming behaviours (O'Sullivan, 2014). Thus, more research is required to inform future education, policy, and practice adequately. 


\section{Youth homelessness}

Paramedics are probably the only health workers who encounter children and young people in every possible environment, including areas used by the homeless and those living on the streets. Fitzpatrick et al. (2017) suggest that both rough sleeping and statutory homelessness are on a sharp upward trajectory; even still, however, homelessness in the UK is thought to be grossly underestimated (Jones and Pleace, 2010). Collating reliable data on homelessness is widely acknowledged as difficult due to the temporality of some people's experiences of homelessness, and the transient nature of homeless individuals, and those who are classed as 'hidden homeless'. Reeve (2011) states that there is no agreed definition of hidden homelessness (which includes those 'hidden' who are using a friend's sofa or spare room, or are in squats), and the term is inconsistently conceptualised and applied. This, in turn, may be problematic in practice, leading paramedics to overlook children and young people who technically are experiencing or are at risk of homelessness.

Homelessness, in general, has been defined as follows: 'Homelessness is literally when one is without a roof', or 'even threatened with homelessness' (Doorway, 2010), or is when 'you can't live at home because of violence or threats of violence which are likely to be carried out against you or someone else in your household' (Shelter, 2016).

Many factors can contribute to a young person finding themselves homeless, such as being thrown out of a family home or parental/carer abandonment (Smeaton, 2009). Other factors include running away from home or care, or drug and alcohol abuse problems (Homeless Watch, 2012). Due to parents with young children being a higher priority, people aged 16-25 are more likely to experience problems with exclusion, entitlement, and visibility when attempting to tackle social issues, thus, making them more susceptible to becoming homeless (Reeve, 2011). Arguably, this increases the possibility of them being treated by community workers such as paramedics, yet there is little literature investigating homeless interactions by paramedics.

North et al.'s (1997) study of A\&E attendances looked at the records of 1,873 homeless service users compared with 28,420 housed service users. This study produced a profile 
which suggested that homeless people were five times more likely to use A\&E departments due to deliberate self-neglect and harm, were four times more likely to suffer assaultrelated injuries, and would have twice the rate of infected wounds in comparison to housed people. Although this study is dated and makes only passing reference to young people, it does indicate the involvement emergency service staff may have with this vulnerable group. It is one of only a few studies which investigate this area of practice, albeit not from a paramedic perspective specifically. Changes in unemployment levels, cuts in benefits, and weakened housing 'safety nets' are most likely to disadvantage young people the greatest and increase youth homelessness (Fitzpatrick et al., 2012).

Given the UK financial downturn of 2008 and the increased propensity homeless people have to seek emergency care, it can be argued that paramedics are more likely to be encountering youth homelessness than in previous years. Although more research is required to support this assertion fully, there is some evidence that suggests homeless people prefer to encounter paramedics than other emergency services. Zakrison et al. (2004), for example, investigated the frequency of interaction between homeless people, police, and paramedics in Canada. They suggested that not only is there a high frequency of paramedic-homelessness interaction, but that homeless individuals trust paramedics far more than they trust the police, and so are more likely to call them in a range of emergencies. While this data is geographically specific and based upon adult experiences, it could be argued that this is likely to be the case in the UK, and more so with homeless youth, who might have had negative experiences with the police, although again, more research is required to support this.

While there appears to be an abundance of literature related to homelessness and use of A\&E departments (Kushel et al., 2002; Pearson et al., 2007; Sadowski et al., 2009; Schneller, 2012), there is a relative paucity of data related to paramedic interactions with homelessness and more specifically youth homelessness. Most of the cited literature about A\&E departments heavily suggests high paramedic attendance to homeless individuals. However, this literature does so in a way that reduces paramedicine to a transportation 
service rather than the first link in the proverbial chain of qualified health professionals who can act in referring, preventing, and highlighting this serious problem. Of the 10.68 million 999 calls received by NHS ambulance services in the year 2015/16, 37.9\% did not result in admission to A\&E and were treated on scene (Association of Ambulance service Chief Executives (AACE), 2016). This limits the applicability of some of the research identified within this review, which focuses on emergency departments only. It is possible that homeless individuals seen by paramedics were some of the nearly 3.7 million patients who did not get transported to $A \& E$; thus, more research is required from a paramedic perspective also. There has been no research identified which relates directly to paramedic perceptions of these patients and paramedics' confidence levels in assessing and treating them; furthermore, there is no specific research into youth homelessness. Without up-todate investigation, it will remain unknown if paramedics are even aware that homelessness is a CP concern for which they are well placed to identify and address. Without this information, future paramedic practice cannot be informed, which is disconcerting given the increase in homelessness being estimated at the time of writing.

\section{Youth substance misuse}

Paramedics are often called to assess and treat those who have abused various substances, including alcohol, illicit drugs, and prescription medication. A proportion of these patients are children and young people. The exact prevalence of paramedic encounters with youth substance misuse is difficult to determine due to the sporadic recording of incidents. While there is a national poisons network from which some data can be extracted (such as search histories), there is no national database for these incidents. This is also compounded by the 'Nature of Call' (NOC) reason, as the cause of the interaction with paramedics may have been substance misuse, but the NOC recorded might be an injury or illness (such as vomiting or a fall). These encounters are unsurprising given that the 2014 smoking, drinking, and drug use among young people survey showed that $8 \%$ of 11 to 15 years had drunk alcohol in the last week and $8 \%$ had been drunk in the last four weeks. Furthermore, $15 \%$ of pupils had recorded taking drugs at various ages (Agalioti-Sgompou et al. 2015). This survey used a multi-stage probability sample of schools and pupils. In all, 522 schools were selected, and 
pupils were selected using a systematic random sampling method that resulted in approximately 35 pupils from across school years 7 to 11 . Due to a lack of funding, there were no surveys undertaken for the years 2015 or 2016. Significant factors associated with drinking and drug use included low well-being, risk-taking behaviours, truancy, exclusion from school, and family influences (Agalioti-Sgompou et al., 2015). It can be argued that this review has shown that paramedics have a role in identifying and referring children and young people involved in drug and alcohol misuse, but again, little research exists investigating this aspect of practice.

There are significantly higher levels of drug use among those young people who belong to more than one vulnerable group, such as those who have been in care or those who have been homeless, have truanted, have been excluded from school, or are serious youth offenders (Becker and Roe, 2005). Consequently, such youths are at a significantly higher risk of suffering anxiety, memory or cognitive loss, sexually transmitted infections, accidental injury, hepatitis, HIV infection, coma, and death. Such consequences transform what may appear to be a youthful Saturday night binge or an experiment with drugs into something much more serious with far-reaching negative biopsychosocial repercussions, which paramedics must recognise. This is particularly important for paramedics to consider given that some of the consequences are likely to involve the need to call 999 and receive urgent or emergency treatment, such as coma, accidental injury, and sudden death.

Gilvarry et al. (2012) suggest that the notion of problematic drug or alcohol use is different for young people than it is for adults in as much as what may be seen as adolescent experimentation in a 17-year-old could be grounds for intervention in a 12-year-old. These views of 'adolescent experimentation' need to be treated with caution by paramedics. It is important not to assume that a drunk teenager is a nuisance; instead, they might be someone who may require intervention for addictive substance misuse and assistance with the potential cause. Paramedics also need to ensure that safeguarding referrals are completed on every occasion of youth drug or alcohol misuse due to the possibility of multiple ambulance crews attending multiple episodes for the same child, but with each 
separate visit being put down to one teenage experiment. There is little research investigating paramedics' knowledge or confidence levels in youth substance misuse. This review has found no research specifically focusing on this area of practice, but has found literature which makes mention of youth involvement in predominantly adult-focused research.

The involvement of adult substance misuse is also important for this review, given that many children and young people who live with substance misusing parents and carers also suffer from neglect and domestic violence and may also be at increased risk of misusing alcohol and illegal drugs themselves (Cleaver et al., 2011). It should also not be forgotten that pregnant females can also require child protective support and referral, given the damage that drugs and alcohol can do in utero. Paramedics need to approach parental substance misuse in two ways. They often have to treat the directly harmful behaviour (such as alcohol and drug overdoses) while also considering that such behaviour can itself be a symptom of perpetrated abuse and neglect. Furthermore, if paramedics are called to an adult who has substance misuse issues, they should always consider the involvement of any children and young people in the family who may suffer from domestic violence.

While literature exists about the medical management of intoxicated youths by paramedics in the forms of guidelines, there is a lack of literature and research about paramedics' thoughts and attitudes towards children and youth substance misuse patients. Glencorse et al.'s (2014) study aimed to ascertain the views, perceptions, and attitudes of paramedics when working with patients presenting with alcohol-related injury or illness. A total of 142 paramedics filled in the questionnaires. This study suggested that levels of commitment, motivation, satisfaction, legitimacy, and adequacy are low in front-line paramedics when working with patients with alcohol-related injuries and illness. However, they are open to finding ways to provide interventions if they are adequately trained. This study made no mention of children or young people and appears to focus on adult patients only, but it does raise the question of whether such negative feelings would restrict the child protection process. 
Paramedics are often the first point of contact with child and youth alcohol and substance misuse patients and are ideally situated to deliver health messages about the possible deleterious consequences (Smart, 2009). Paramedics are also often the first point of contact with children and young people who are more susceptible to progressing to substance misuse (domestic abuse, truancy, homelessness), and so can play a part in identifying and possibly negating further problems. Despite this, there is little research investigating paramedics' thoughts, knowledge, understanding, and confidence levels when dealing with such patients. Without this research, paramedic practice, policy, and education cannot be fully informed. Equally, without highlighting the involvement paramedics have in child and young person practice to other professions and disciplines, ambulance services and paramedics may not be held to account for any failings or limitations in practice, and thus cannot then learn and improve service delivery and patient care.

\section{The prevalence of abuse neglect and child death within the UK}

WHO (2010 A) estimates that there are around 31,000 homicides of children under the age of 15 each year globally and further estimates that around $20 \%$ of women and $5-10 \%$ of men report having been sexually abused as children. Despite these estimates, there are no entirely reliable global figures for the prevalence of child maltreatment. Data for many countries is lacking, especially low-middle-income countries. This makes studying this already difficult and complex subject even more problematic. From a UK perspective, the Office of National Statistics (ONS) (2015) compendium on Violent Crime and Sexual Offences showed that in the year ending March 2015, children under 1-year-old had the highest rate of homicide (35.8 offences per million population) compared with other age groups.

Children aged under 16 also account for a large proportion of rape offences recorded by the police. Just under a third of female rape victims were aged under 16 with the majority (60\%) of male rape victims being aged under 16 also. These figures are from the crime that has been reported or detected and may not show the true scale of the problem; which remains largely unknown. 
While it will always be difficult to determine exact figures, not least due to the hidden nature of neglect and abuse, the NSPCC (2016) produced a report titled How Safe are our Children?, the most comprehensive overview of child protection in the UK 2016. This report provided an overview of the child protection landscape and compiled the most up-to-date child protection data that exists across each of the four nations in the UK. Some of the key elements pertinent to this thesis are shown below:

- There were 75 child homicides across the UK in 2014/15

- Police recorded 47,008 sexual offences against children in the UK in 2014/15 and 10,136 cruelty and neglect offences against children aged under 16 in the UK in 2014/15.

- Over one in six 11 - 17-year olds have experienced some type of severe maltreatment.

This data highlights that child protection in the UK is still very much an issue and supports the need to ensure that all HCPs who encounter children within their work understand their roles, so they can aide detection and prevention. It is not known if UK paramedics understand the extent to which child maltreatment is still an issue within the UK; no results were found as part of this review which investigated this line of enquiry. Figure $\mathbf{3}$ shows the number of children on child protection registers or subject to a child protection plan on 31 March (or 31 July in Scotland) 2015 (NSPCC, 2015).

Figure 3: Child protection statistics UK: $2011-2015$

\begin{tabular}{l|lllll} 
Nation & $\mathbf{2 0 1 1}$ & $\mathbf{2 0 1 2}$ & $\mathbf{2 0 1 3}$ & $\mathbf{2 0 1 4}$ & $\mathbf{2 0 1 5}$ \\
\hline England & 42,700 & 42,850 & 43,140 & 48,300 & 49,690 \\
Scotland & 2,571 & 2,698 & 2,645 & 2,877 & 2,751 \\
Wales & 2,880 & 2,890 & 2,955 & 3,135 & 2,935 \\
Northern & 2,401 & 2,127 & 1,961 & 1,914 & 1,969 \\
Ireland & & & & & \\
\hline UK & $\mathbf{5 0 , 5 5 2}$ & $\mathbf{5 0 , 5 6 5}$ & $\mathbf{5 0 , 7 3 7}$ & $\mathbf{5 6 , 2 3 1}$ & $\mathbf{5 7 , 3 4 5}$
\end{tabular}


Knowing if a child patient or a child of an adult patient is subject to a child protection or child-at-risk plan is important. This should form part of a paramedic's history taking, but little is known regarding the extent to which paramedic enquire about this information, or indeed what information ambulance services store. Many reports and enquiries cited within this review have referred to the need to share data effectively with partner agencies. With ambulance service control rooms using computer-aided dispatch (CAD) systems, it might be possible to integrate this kind of information so that paramedics are aware of children at various addresses who are subject to these plans. This has clear limitations when children or parents of children are at different addresses and give different spellings of their name, in error, or fraudulently, but such problems might be mitigated by the Child Protection Information Sharing system.

The Child Protection Information Sharing system is intended to be a nation-wide computer programme that aims to avoid any repetition of cases of abuse, such as Baby P. It aims to provide a method by which specific information can be shared between social care and unscheduled healthcare settings with regard to child protection, and aims to help improve decision-making around children who are being abused and/or neglected. It is also meant to be a solution to deal with issues of the migration of children across local boundaries where they are not known in other areas, which can be done on purpose to avoid detection. This new system is expected to be available to clinicians working in all unscheduled care settings, such as A\&E, out-of-hours GPs, and walk-in centres, by 2018 (Health \& Social Care Information Centre (HSCIC), 2015). This system will highlight repeat admission and will also highlight those children on child protection or child-at-risk registers and so may assist in the detection of child protection data, clinicians will be better able to reduce modifiable factor child deaths, which we continue to explore.

Neglect is the most common reason for a child being subject to a child protection plan or being placed on a child protection register (CPR) in England and Wales, and it is a form of abuse which often involves modifiable factors. In Northern Ireland, however, physical abuse has overtaken neglect as the most common cause of being on a CPR. If HCPs miss neglect and abuse, the serious case review (SCR) investigation could record that there were 
'modifiable factors' involved in the child's death. Modifiable factors are events, actions, or omissions that contribute to the death of a child and which, using interventions, could be modified and avoided (Mahajan et al., 2013). The NSPCC (2016) judges modifiable factors to be anything that could be avoided by using a nationally or locally achievable intervention that is recognised to reduce the risk of a child's death. Examples of modifiable factors are an inability to recognise danger signs, maternal ill health, treatment by quacks/faith healers, delay in the detection of signs, delayed or omitted referral by the treating team, shortage of staff, lack of specialised care, and communication gaps between staff.

Figure 4 shows the number of Child Death Reviews in the year ending 31 March 2016 with the percentage of modifiable factors by category of death.

\section{Numbers of deaths reviewed and percentage with modifiable factors by category of death}

Category of death

Sudden unexpected unexplained

Trauma and other external factor

Suicide or deliberate self-inflicted

Deliberately inflicted injury abuse or neglect
Number of deaths

2,978

276

186

119

57
Percentage with modifiable factors

$16 \%$

$65 \%$

$56 \%$

$42 \%$

$60 \%$

Department for Education (2016) 'Child Death Reviews - Year ending 31 March 2016'. Department for Education. London. 
While all child deaths are reviewed, not all child deaths are subject to an SCR. An SCR will be initiated where

a. abuse or neglect of a child is known or suspected, and

b. either-

1) The child has died.

2) The child has been seriously harmed, and there is cause for concern as to the way in which the authority, their Board partners, or other relevant persons have worked together to safeguard the child's welfare.

(Department of Education, 2016)

In the year ending 31 March 2016, 2,458 of the deaths reviewed occurred in an acute hospital and 128 in a hospice. Not all deaths which result in a serious case review will be assessed as having modifiable factors. An SCR was carried out for 3\% of all deaths reviewed in the year 2015-16, and of these deaths, $53 \%$ were deemed to have modifiable factors, compared to $79 \%$ in 2014-15. Child deaths that will be reviewed as a matter of course but might not involve modifiable factors are those of children with life-limiting conditions or known medical problems, and neonatal deaths (Department of Education, 2016). It can be argued that modifiable factor deaths can be reduced if professionals such as paramedics were to work to their full potential. There has been no data identified as part of this review which specifically investigates paramedics as part of modifiable factor in child deaths. However, it is likely that in the many SCRs each year in the UK paramedics may have been involved. More research is needed to understand if UK paramedics fully understand their role in reducing child deaths in the UK, and, furthermore, it is important for SCR panels, academics, and policymakers to understand the roles that paramedics play so that current and future multidisciplinary practice can be informed and improved. 


\section{The history of child protection}

Bilston (2004) posits that while the practice of child abuse (as we define it today) has occurred throughout all human history, it is only in the last century or so that it has been recognised as a distinct phenomenon and something from which children have a right to be protected. The recognition that children have the right to be protected from harm means that child abuse has become a legal, medical, and social matter, and as such, has been the subject of much research. An early example of such research was Kemp's 'battered child syndrome'. In his paper titled The Battered Child Syndrome (Kempe et al., 1962), it was shown how $\mathrm{x}$-ray evidence could prove the repetitive physical abuse of children. Through his research, Kempe further conceptualised child abuse as a medical problem (Munro, 2008), but despite his continual advertisement of child abuse (using the term 'battered child' to garner maximum emotional public attention), various socio-political and professional barriers limited his work, for example, the lack of defined responsibility amongst professionals. Hobart and Frankel (2009) stated that throughout the 1960s, child abuse in the UK remained largely within the realms and responsibilities of GPs and casualty officers alone. Improvements in forensic medicine and closer cooperation between medical, legal, and social services led to a greater understanding of child abuse and a recognition that it is everyone's responsibility to prevent it, including, more recently, paramedics.

\section{Landmark cases}

Many landmark cases have identified failings by multiple agencies in their identification, prevention, and treatment of, and subsequent reaction to incidences of various levels of abuse and neglect. Notable cases have been the impetus for historical changes in the way practitioners and organisations approach child protection within the UK, and much can be learned from them. The case of 8-year-old Mary Ellen Wilson is widely accepted as being the catalyst for the creation of child protection movements and the introduction of laws to protect children from maltreatment by caregivers (Lamont and Bromfield 2010). In 1874, a church social worker saw that Mary was repeatedly starved and beaten by her stepmother. Finding that no person was willing to support her case to protect Mary, she resorted to 
using animal protection laws to gain a prosecution. After the social worker argued that Mary had the same rights as an animal to be treated humanely, her stepmother was imprisoned for cruelty (Shelman and Lazoritz, 2005). This case is now credited with the production of the American Society for the Prevention of Cruelty to Children, and nearly a decade later, the London (now national) Society for the Prevention of Cruelty to Children (NSPCC), which was modelledon the American version. The case of Mary Ellen Wilson was the first of many examples of a change to the way in which children were viewed within society and rights afforded to them. Subsequent changes saw the introduction of the 1908 Incest Act, the 1933 Children and Young Persons Act, and the 1948 Children Act. These acts forced local authorities to create the role of a child care officer (Munro, 2008), which was a role concerned not only for children's development but the entire family structure. Landmark cases (examples of which can be found in Appendix 1) have unarguably been the impetus for much social, procedural, and legal change within the UK. How these changes have affected the role of the paramedic within the child protective process is not fully understood, and this is likely to remain the case until research is conducted into paramedics' thoughts, perceptions, and roles in child protection. Whitnell (2010) highlighted this lack of paramedic child protection-related research and reminded paramedics that despite the absence of research, they are still in an extremely valuable and important position to identify and protect children and young people.

What is noteworthy is that throughout many landmark SCRs, the role of the paramedic appears not to have been considered. Within the Climbié inquiry, for example, the only mention of paramedics was about the ambulance station Victoria was taken to by a taxi driver and her subsequent transportation to the hospital ${ }^{7}$. Similarly, the involvement of the ambulance service and thus the paramedics in the review of Baby P's death only mention them in relation to resuscitation and transportation ${ }^{8}$. The review of Daniel Pelka's case does

\footnotetext{
${ }^{7}$ London Ambulance Service when asked in a freedom of information request stated that they could not categorically confirm that paramedics had not seen Climbié more than once prior to her death.

8 London Ambulance Service when asked in a freedom of information request stated that they could not categorically confirm if paramedics had seen Baby $\mathrm{P}$ more than once prior to her death.
} 
state some previous involvement by paramedics with his mother, but again makes little to no mention of the wider role paramedics played, if any, in previous interactions, assessments, and treatment of Daniel before his cardiac arrest and subsequent death ${ }^{9}$. It is possible that paramedics were considered within these reviews, but their involvement was not formally recorded within the final written report. Considering the number of injuries sustained by Climbié, Baby $P$, and the ongoing neglect Daniel Pelka suffered, one questions if paramedics correctly reported or referred any concerns to the authorities and professionals. The answer to this question appears to have been omitted from multiple SCRs and remains unanswered by freedom of information requests submitted by this author. Without knowing these answers, it is difficult to assess the efficacy of the paramedics' involvement and to assess further if the education and policy that supported their practice was adequate or perhaps required improvement. Such omissions in many SCRs might suggest that they may overlook the role paramedics play in child protection; however, without systematic investigation of all UK SCRs, this assertion cannot be fully supported. Unless paramedics are considered as part of the child protection process, their actions cannot be scrutinised to the degree to which they should be, meaning lessons cannot be learned. This is not the case, however, for all SCRs.

Although many SCRs either do not consider or do not show any evidence of their consideration of the role of paramedics, the review into the death of Hamzah Khan was different. Hamzah Khan was a child who died because of maternal neglect in 2009, but whose body was not discovered until 2011. The SCR highlighted the role the ambulance service and paramedics played within the child protection process. Not only did the review comment that West Yorkshire Ambulance Service submitted evidence to the SCR, but it also made mention of "attendances at A\&E" and stated that "one such attendance was via an ambulance that had been called to the house by one of the children" (Serious Case Review:

\footnotetext{
${ }^{9}$ West Midlands Ambulance Service can confirm only that their paramedics had contact with Daniel Pelka on the day of this death, after which they completed a child protection referral. However due to data protection they cannot confirm if they had ever attended the address, treated, or made referrals for any other person than Daniel, in the past. They did not state how many times they had seen Daniel in the past also.
} 
Hamzah Khan, 2013). By scrutinising the role of the paramedics in the death of Hamzah Khan, it was concluded that the ambulance crew ensured that their concerns were reported to Children's Social Care Services. The review further highlighted that 'ambulance services might have significant information about families which is relevant to multi-agency enquiries that are not routinely sought' (Bradford Safeguarding Children Board SCR Executive Summary 2013 Hamzah Khan Section 1.8 (49) P). This point has previously been mentioned within Kirtley's (2013) review of CSE and supports the need for the ambulance service and paramedics to be involved not only in the referral process, but also in the investigation and reflection process. Such landmark cases, along with many less well-known cases, have been the impetus for investigation, reflection, and reform in social attitudes, policy, and legislation over the decades, an issue we will now explore in greater depth.

\section{Reforming the UK child protection policy: The Children Act 1989 to present day}

\section{The Children Act 1989}

The Children Act 1989 was introduced to reform and clarify the plethora of existing laws affecting children and young people. At the time, it was hailed as the most comprehensive and far-reaching reform of child law (NSPCC, 2012) and is the Act upon which the current child protection system is based. The main principles and provisions embodied in this legislation include ensuring that the welfare of children must be the paramount consideration when courts are making decisions about them, and that parental/carer responsibility has replaced the concept of parental/carer rights. Also, local authorities are charged with the duty to identify children in need and to safeguard and promote their welfare. Orders under this Act should not be made unless it can be shown that the proposed action or outcome will be better for the child than not making an order (Children Act 1989). The 1989 Children Act emphasised the importance of the prevention of harm, the promotion of effective care, and the importance of holistic wellbeing of all children. All three of these aims are the responsibility of paramedics, but like with many other other pieces of legislation, little literature exists to reflect this (Whitnell, 2010). 


\section{The UN Convention on the Rights of the Child 1989}

The United Nations (UN) ratification of the Convention on the Rights of the Child (UNCR) (1989) was a landmark development in the progress for children's rights. UNICEF (2013) described its ratification as the most complete statement of children's rights ever produced, and the most widely-ratified international human rights treaty in history. The convention included the right to have a childhood, to be educated, to be healthy, to be treated fairly, and to be heard (UNCRC, 1989). This convention applies to all members of society and those in positions of responsibility to care, such as paramedics. There is little literature available focusing specifically on paramedics' responsibilities under this convention; however, it is mentioned in textbooks such as Foundations of Paramedic Practice (Blaber, 2012) in a historically descriptive manner. One questions how well paramedics understand their legal responsibilities under the UNCRC, and how these affect their day-to-day practice. Towards the latter end of this thesis being written, the UK decided to leave the European Union (EU). Nonetheless, the UNCR will continue to apply to the UK after its departure from the EU, and so it is important to consider whether paramedics know this and if this is covered in any updated training.

\section{Every Child Matters 2003}

Following Victoria Climbié's death in 2000 and the subsequent review in 2003, the UK government launched the initiative Every Child Matters (ECM). ECM was described by the then Prime Minister Tony Blair as a range of measures to dramatically reform and improve children's care in the UK. It aimed to support parents and carers and to provide early intervention and effective prevention of harm. This review of the literature has identified that paramedics have a role to play in the early intervention and effective prevention of harm; however, they are not mentioned within ECM. This point is noteworthy because of the emphasis placed within ECM on community-based initiatives and the roles of similar professions, such as the police, doctors, nurses, and health visitors. This initiative is now 15 
years old and although still pertinent, is somewhat dated; later initiatives do consider the role of ambulance services and paramedics.

\section{The Children Act 2004}

The Children Act 2004 provided a legal framework for the changes set out within ECM (2003) and amended the Children Act 1989. The Act created the post of Children's Commissioner for England (Wales already had one) and prescribed the organisational restructuring and information processing systems needed to encourage professional collaboration and data sharing (Munro, 2008). Eight to ten years later, both Kirtley (2013) and the SCR of Hamzah Khan (2011) highlighted the lack of data-sharing involving ambulance services and paramedics. This demonstrates that solely creating legislation does not necessarily change practice and that legislation alone cannot be relied upon to ensure the protection of children and young people. The Children Act 2004 emphasises the importance placed upon integrated working processes and procedures between all agencies; and places a legislative duty on 'relevant partners' to co-operate and share information (NSPC, 2010). It can be argued that due to the lack of any mention of paramedics within ECM (2003), paramedics have been overlooked as 'relevant partners', thus justifying the need for more research into the roles they do play within child protection.

\section{Working Together to Safeguard Children 2006, 2010, 2013, 2015, 2018.}

This emphasis on expanding and including all potential partners in child protection is again noted and reiterated in the later government initiative, WTSC (2006). Revised in 2010 and 2013 and once again in 2015, WTSC sets out how organisations and individuals should work together to safeguard and promote the welfare of children and young people in accordance with the Children Act 1989 and 2004. Each subsequent publication updates the previous version, and while each version is not a major review of the previous one, it incorporates guidance on the newest legislation. Importantly for paramedicine, and a turning point in the recognition of the paramedics' role within the community, all four versions of WTSC cite the role of ambulance services and thus, paramedics. WTSC (2006), for example, states that 
ambulance staff may be in a position to identify initial concerns regarding a child's welfare. WTSC (2010) recognises that ambulance staff will have access (by phone or in person) to family homes and will be involved with individuals at times of crisis. This emphasises the need for a multi-professional approach to ensure collaboration among all involved, including ambulance staff (WTSC 2010). Each WTSC publication argues that it is important for all parties involved to understand their responsibilities and duties as set out in the primary legislation. However, there is no literature pertaining specifically to paramedics that investigates this level of understanding, or paramedics' perception of such responsibilities. Given that paramedics are included within these legislation-based government initiatives, the lack of research surrounding them is disconcerting; research needs to be undertaken to ensure that paramedics are meeting their professional and legal obligations.

\section{Protection of Children in England: A Progress Report 2009}

Following the Baby P case, Lord Laming authored a report titled The Protection of Children in England: A Progress Report. While the report highlighted some of the progress made in relation to ECM (2003), however, it also highlighted the challenges facing child protection services and the significant problems in the day-to-day reality of working across organisational boundaries and cultures. Interestingly, despite Laming (2009) making significant mention of A\&E departments, he says nothing about how the child was referred or transported to A\&E. There is no mention of paramedics in comparison with the police, GPs, and nurses despite the similarities in paramedics' work. This omission suggests an underestimation of the role of paramedics and an underestimation of their experience of witnessing the atmosphere and interactions within the home environment. The only reference to paramedics is made in passing and refers to the need for professionals to understand the respective roles, responsibilities, and procedures of each agency involved in child protection. Laming states that the scale and quality of multi-agency training needs to be substantially improved to ensure that organisations such as early years' providers, schools, social workers, paramedics, and others who step in when problems arise have an understanding of these roles (Laming, 2009, p. 56). This review of the literature has not identified any research into the efficacy of UK paramedic CP training, and Laming himself 
does not cite any research that supports the assertion that training needs to be improved. While it might be the case that training does need to be 'substantially improved', without a credible evidence base, it is difficult to support such a statement. A credible evidence base resulting from high-quality research will inform future paramedic practice, policy, and if needs be, training also.

\section{The Munro Review 2010, 2011, 2012}

In 2010, Professor Eileen Munro undertook the first of three reviews of UK child protection systems. Thought to be the foundation upon which the most modern of child protection systems are now based, the Munro reviews analysed, criticised, and made recommendations to all those involved in areas concerned with child protection (health, education, social work). The first report was purposefully analytical and made no major recommendations. It found that in recent times, child protection services had been shaped by certain forces which came together to create a defensive system that puts too much emphasis on procedures and recording, and not enough emphasis on developing and supporting the expertise to work effectively with children, young people, and their families. The following two reports set out proposals for reform which were intended to create the conditions that would enable professionals to make the best judgments about the help to give to children, young people, and their families. Munro's reviews criticised the overbureaucratization of simple services aimed at protecting and nurturing children and young people and stated, "Too often in recent history, the child protection system has, in the pursuit of imposed managerial targets and regulations, forgotten that its raison d'être is the welfare and protection of the child" (Munro 2011 p. 8).

Munro (2012) concludes that by decreasing the bureaucracy within current systems and by supporting staff to develop professionally, the quality of child and family social work could improve. Like previous UK-based reports, A\&Es, the police, nurses, and doctors were all mentioned; however, ambulance services were not. Given that the terms 'frontline 
practitioner' and 'community' are used so very often in these reviews, how is it possible that paramedics and ambulance clinicians were not considered? Paramedics are community frontline practitioners whose roles appear to have been underestimated in recent times.

Each review cites a wide range of research evidence and often recommends undertaking further research to understand practice better. Given that one of the methodologies employed by these reviews and other similar reports tends to be the systematic review of the literature, perhaps it is quite explainable that paramedics and ambulance services are not considered. The lack of available research evidence focusing on paramedics possibly leads to their omission from reports and reviews and perpetuates the underestimation of their role within CP practice. Munro calls for more research and reiterates the need to encourage the use of research evidence and professional experience to support and improve practice. Currently, there is a lack of this research evidence supporting paramedic practice, and little is known about the professional experience of paramedics working with children and young people. More is needed to support the future practice, policy, and education.

\section{Children and Families Act 2014}

Many of the previous examples of legislation and guidance include the need for families and health and social care providers to work more closely to achieve outcomes. The Children and Families Act 2014 aimed to take forward the commitment of the coalition government at the time to improve services for vulnerable children and to provide stronger support for families in a more cohesive and family-orientated way. It covers a wide range of areas including adoption, childcare, child welfare, family justice, parents' rights, and children and young people with special educational needs and disabilities. The basic goals are to give families a greater involvement in decisions about their support and to encourage social care, education, and health services to work together more closely in supporting those with special needs or disabilities (Donovan, 2014). Although not widely advertised, the professionals on whom this Act focused have synonymous roles to those of paramedics in 
their work within the community. The well-being element of this Act highlights the importance of paramedics understanding the need for integrated working and information sharing. Well-being is not considered to be solely positive physical, emotional, and mental health but also protection from abuse and neglect, and positive domestic, family, and personal relationships along with social and economic well-being. Whilst much of this Act tends to focus on social and primary care providers, paramedics can contribute to positive child and family well-being by recognising the need for reporting or referring concerns, as well as having open discussions with care givers about the support they may require to achieve these outcomes.

\section{Learning from history and reform}

What is evident throughout the analysis of the recent reforms, reviews, and legislative changes that have shaped child protection within the UK is that the role of paramedics remains underestimated, misunderstood, and somewhat forgotten. Some, but not all of the literature included within this element of the review mentions paramedics and their wider role within community $\mathrm{CP}$ but does so insufficiently in comparison with other professions such as policing and nursing. ECM (2003), the Children Act 2004, and the Munro reviews $(2010,2011,2012)$ are all pivotal documents that shape child protection in the UK yet fail to consider or evidence the consideration of ambulance services or paramedics. WTSC (2006, 2010, 2013) and "Protection of Children in England: A Progress Report" (2009) are equally pivotal, yet only mention ambulance services or paramedics in passing and provide no evidence of the understanding of their role. Parton (2004) claims that over the previous 30 years, the frontline professionals, organisations, and agencies who had responsibilities for protecting children and families were quite incapable of learning much-needed lessons, and crucially, of putting them into practice in such a way that would avoid repeating past tragedies. This point was reaffirmed through the SCRs of Baby P and Daniel Pelka and highlighted how failings by both the system and those who work within it continue to occur. Reasons for this include poor communication, a poor understanding of risk, and a lack of collaboration and data sharing. Parton (2004) further states that recommendations and reforms should not solely be pertinent to the HCPs that most commonly work with 'at-risk' 
children, but should be pertinent to any HCP who has the potential to be involved in the care of a child or young person. This review of the literature has identified paramedics to be such professionals, but has also identified a lack of research investigating their understanding of risk, amongst other things. This research evidence is needed to support and improve current and future paramedic practice.

\section{Child Protection: Paramedic Training and Education}

UK ambulance services have policies and frameworks which outline paramedics' responsibilities to safeguard children and young people. Identification of risk and onward referral are an integral part of these policies; however, unless paramedics can successfully identify risk, or recognise harm and vulnerability, they will be unlikely and often unable to refer children and young people in need. These policies and frameworks reflect the legislation that underpins them and set out the statutory duties placed upon HCPs working with children. The Children Act 2004, for example, and specifically, section 11 places duties upon ambulance services and those who work for them to ensure that they consider the need to safeguard and promote the welfare of children when carrying out their functions (Children Act 2004 Section 11.2a). The Act recognises that HCPs are in a strong position to identify welfare needs or child protection concerns, and where appropriate, can provide support (WTSC, 2015).

To ensure compliance with the legislation, ambulance services must provide training and education to their staff. WTSC (2015) provides statutory guidance for those working with children and states that employers are responsible for ensuring that their staff are competent to carry out their responsibilities for protecting and promoting the welfare of children. This review found no research specifically investigating the efficacy of ambulance service $\mathrm{CP}$ training or the competency of ambulance service staff, such as paramedics. The NSPCC (2012) recognises the role that paramedics play in child protection and states that they are often uniquely placed to help safeguard children from abuse and neglect during 
home visits and contact with families through which they gain an insight into children's everyday lives (NSPCC, 2012). It further states that it is therefore vital that paramedics be given high-quality training on how to recognise and respond effectively to signs of child abuse and neglect. However, this review has found no research investigating the efficacy of $\mathrm{CP}$ training for paramedics, and nor has any research been found which specifically investigates paramedics' confidence levels in their training.

Child protection training in the UK tends to be provided in all ambulance services at an introductory level during an employee's induction period. Further training is provided throughout the employee's career, but this can vary dramatically from service to service. Variances include which grade of ambulance staff undertake this training, who delivers it, and how often it is delivered, all of which may affect the quality of its use in practice. Examples of training are

- online training

- computerised video / DVD simulation training

- self-directed workbook completion

- classroom sessions

- reflection sessions (clinical supervision) ${ }^{10}$

Introductory training appears to be delivered to all new employees regardless of their background, which is positive. Most paramedics entering the profession are now university educated, and CP, among other related subjects, appears to be included in their undergraduate curriculum. However, this review of the literature has found no research into the national consistency or efficacy of this form of education, or into assessing how competent students are deemed after completion. Jones et al.'s (2010) mixed methods study highlights how some student paramedics viewed subjects such as sociology and

\footnotetext{
${ }^{10}$ Clinical supervision is a recognised and validated activity in healthcare that brings skilled supervisors and practitioners together in order to reflect upon their practice. It is a facilitated and structured time for practitioners to think about their knowledge and skills and how they may be developed to improve care.
} 
psychology far less favourably in comparison with more practical subjects, such as trauma and resuscitation. Some students even considered such sciences as superfluous to their paramedic education, despite evidence suggesting otherwise. One questions if $\mathrm{CP}$ is considered in a similar vein, and if so, to what extent. Jones et al.'s (2010) study did not, however, specifically investigate how confident student paramedics were in these subjects. Similar results were seen in Roberts and Henderson's (2009) research with qualified Australian paramedics with mental health cases. Paramedics felt confident in their abilities to assess and manage physical trauma, but less so with mental health patients. Students in Jones et al. (2010) reported trepidation associated with attending emergencies involving children, but this appeared to relate to children who had been severely injured or were dying. While these findings give an insight into student paramedics' education, more clarity is needed to determine if this perceived hierarchy of knowledge applies to CP.

\section{UK voluntary ambulance service child protection education}

Multiple voluntary ambulance services within the UK provide first aid cover at public events, routine pre-planned patient transport journeys, and at times, support for NHS ambulance services. These organisations also have a duty under Section 11 of the Children Act 2004 and as such, should have adequate policies in place also. The literature from British Red Cross (2016) and the literature from St John Ambulance (2016) approach child protection from two perspectives: firstly, recognising risk to those children directly receiving their services (first aid), and secondly, identifying those children within the organisations, such as youth members. Both organisations appear to have policies in place to address their duties under the Children Act 2004; however, no non-publicly funded ambulance service was willing to share their policies for this review of the literature. As such, this author is unable to confirm if such organisations do have policies in place or if they appear adequate in comparison with their legislative duties and other ambulance services. 


\section{The psychosocial aspects of paramedic practice}

In 2000, the DoH introduced national reforms to the curricula of all health staff, aiming to 'give everyone working in the NHS the skills and knowledge to respond effectively to the individual needs of patients' (DH, 2000 p44). This move saw paramedicine transition into higher education and question more critically its true workload. Bradley (2005) later highlighted that only $10 \%$ of those calling 999 have a life-threatening emergency and that most calls received yearly are of a sociological and psychological origin. He argued that paramedic education should mirror this data and focus less on the management of lifethreatening emergency situations. This estimate is now over 13 years old, and no reliable, up-to-date data can be found to evidence the number of calls to UK ambulance services which are still sociological and psychological in origin. Of the 10.68 million 999 calls received by NHS ambulance services in the year 2015/16, 37.9\% were treated at the scene, and $10.2 \%$ were treated over the telephone (AACE, 2016); this suggests an estimate of $48.1 \%$ of calls which are not life-threatening. Demand for all UK ambulance services during 2015/16 continued to grow, with a $15 \%$ increase over three years and a $42 \%$ increase over the past eight years (AACE, 2016). It is not known how many of these 999 calls involved children or young people, but the general demand for ambulance service is envisaged to increase further over the next 15 years and may be indicative of the previously projected increase in the UK population (Wanless, 2006).

This increase in population is thought to be made up of both older people living for longer (Lyons and Patterson, 2009), and increases in immigration (Garcia et al., 2012). These two groups of society are often vulnerable and in need of increased levels of social support. This may mean that already stretched social services are further stretched, and the level of available support for all, including children and families, may be reduced (Kings Fund, 2015). This position may be further compounded by the recent globally recognised financial crisis of 2008 (Appleby et al., 2009) and the ongoing austerity cuts to health and social care 
budgets (Kings Fund, 2015). Reductions in access to social care may lead to episodes of medical and psychosocial crisis involving adults and children, to which paramedics may be called. Indeed, crises to which paramedics may be called are closely linked with modern-day poverty. There are currently 3.7 million children living in poverty in the UK, with 1.7 million of these children living in severe poverty (Barnardo's, 2017). Poverty is linked to domestic overcrowding, depression, suicide attempts, household accidents, dehydration, starvation, and homelessness, all of which can affect child welfare, yet to which there seems to be no reference within the paramedic literature (Brady, 2012).

Blaber (2012) states that paramedics work in a unique sense in comparison with office- or hospital-based clinicians. Paramedics see people in their own social world and can observe interactions and behaviours that others will be unable to. As a result, paramedics should have a wider understanding of psychology and sociology and a better understanding of why people talk and act in certain ways. Paramedics should understand why and how people respond to illness and injury, why groups of people may be more susceptible to certain challenges in life, and how this can affect children and young people.

There is little research investigating how paramedics perceive this important social and physiological role, or how they may prioritise any psychosocial responsibility they may have with the provision of medical care. Jones et al. (2010) investigated student paramedics' experiences of higher education and revealed that students felt that their main role was that of treating critically ill and injured medical patients more so than other patient groups. Similarly, Campbell and Rasmussen's (2012) ethnographic study in Canada suggests that paramedics often encounter families in crisis and vulnerable living situations, but that these situations are just noted rather than actually attended to because of a focus on medical responses. Through informal discussions, Campbell and Rasmussen highlight that paramedics felt such situations were beyond their training, mandate, and capacity to respond to effectively. There is no recent UK-based research which investigates this specifically. However, parallels can be drawn from this study. Campbell and Rasmussen's research is very general in its findings and does not focus greatly on specific psychosocial 
determinants of health and wellbeing (as seen in Figure 5). However, Smith et al. (1998) state that most evidence suggests that material conditions are the underlying root of illhealth. With poverty imposing constraints on material conditions of everyday life, there is often limited access to the fundamental necessities of good health, such as adequate housing, good nutrition, and the opportunities to participate in society; all factors termed as social determinants of health ${ }^{11}$.

Figure 5 - Social Determinants of Health

\section{Determinant of health Description}

\begin{tabular}{l|l}
\hline Adequate Housing & $\begin{array}{l}\text { Inadequately heated homes harbour mould and damp, which can lead } \\
\text { to acute and chronic conditions, such as hypothermia, infections, } \\
\text { headaches, pains, and lifelong respiratory conditions (Wilkinson 2009). } \\
\text { Chronic conditions can lead to poor school attendance for children and } \\
\text { thus deficient educational attainment. Poorer houses tend to be } \\
\text { overcrowded, and less likely to be able to host guests, leading to high } \\
\text { levels of social isolationism, developmental delay, depression, } \\
\text { aggression, relationship tension, and poor childhood mental health } \\
\text { (Blackman, 2008 }\end{array}$ \\
\hline Good Nutrition & $\begin{array}{l}\text { Poorer households tend to have poorer nutrition, with food high in salt } \\
\text { and fat, a factor which is implicated in many chronic pathologies, such } \\
\text { as heart disease, diabetes, hypertension, cancer, and strokes (Shi et al. } \\
\text { 2009). Such foods are more affordable, last longer, and can fulfil the } \\
\text { emotional needs of children (Caraher and Coveney 2004). }\end{array}$ \\
\hline $\begin{array}{l}\text { Poor nutrition is further compounded by a poor national uptake of free } \\
\text { school meals (Farthing 2012) within the UK, leading to abnormal } \\
\text { growth, poor development, and a lack of concentration as well as } \\
\text { childhood obesity. }\end{array}$ \\
\hline $\begin{array}{l}\text { Poorer families tend to suffer from social exclusion, as low amounts of } \\
\text { disposable income limits participation. Social exclusion and the inability } \\
\text { to afford to attend education excursions, after-school clubs, and } \\
\text { sporting events, hamper effective psychosocial and cognitive } \\
\text { development, growth, and the ability to form meaningful relationships. }\end{array}$ \\
\hline
\end{tabular}

${ }^{11}$ A prime example of this is that there is a decrease in life expectancy by one year for each of the six stops on the London Underground if one travels from Tower Hill into the less affluent area of the East End (Shaw 2005). 
Berkman (2009) suggests that such health disparities can be reduced if there is a better understanding of the forces that determine the magnitude of the differences among different social, economic, and racial or ethnic groups. Furthermore, Rhodes and Pollock (2006) posits that emergency medicine is well positioned to bridge biomedical and public health approaches for preventing disease and injury, and for promoting health through population-based strategies targeted at the community. Paramedics are the emergency medical staff who currently play a key role in recognising and referring children and families who are experiencing such challenges, and thus they have a key role to play in community health promotion. This review of the literature has found no data investigating UK paramedics' knowledge of psychosocial determinants of health or their perception of such concepts, which is a significant finding due to the identified role. The conditions in which people live, the quality of their food, and their level of social participation are all important contributory factors to good health. Paramedics need to understand these concepts to recognise when any resulting crisis, neglect, and harm is likely to occur.

Brady (2012) suggests (as illustrated in Figure 6) that physical, psychological, and social factors are intrinsically linked; if one factor is affected, then all others are likely to be also. When presented with a medical need for a child or family, social and psychological needs or causes should also be considered. This view remains at the heart of the WHO's (1948) definition of health:" Health is a state of complete physical, mental and social well-being and not merely the absence of disease or infirmity".

Despite this well-recognised view, there remains a lack of literature examining the social and psychological content of paramedic practice and curricula in comparison with the physical/medical, especially from a child and young person perspective. Some literature does, however, focus on adult self-harm, care of the elderly, and adult domestic abuse. 


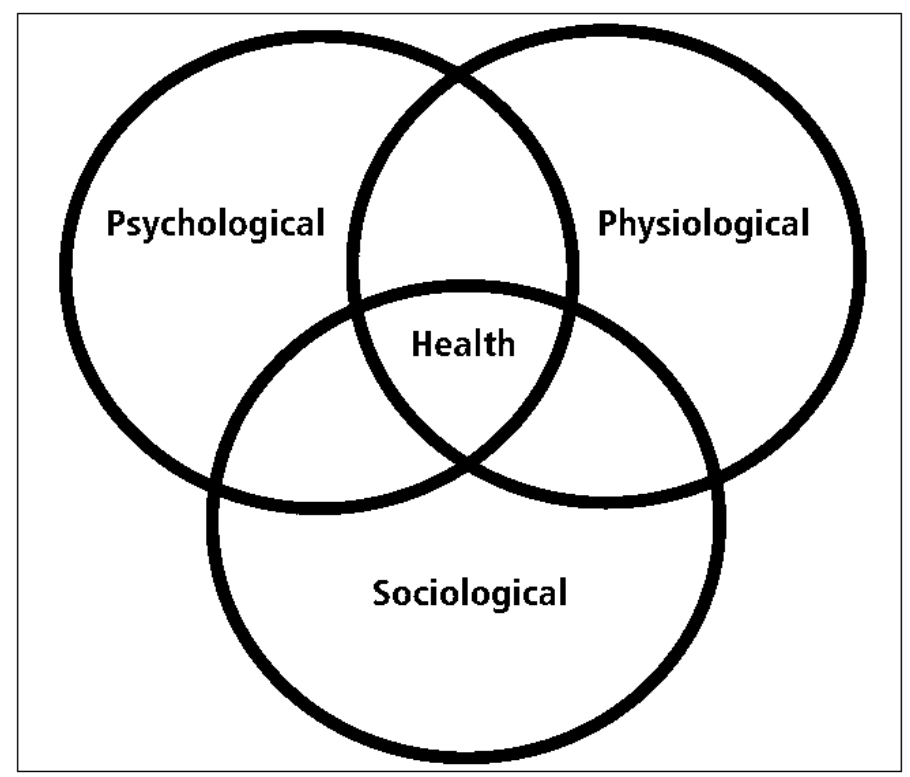

Parallels can arguably be drawn between the work undertaken by community psychiatric nurses and social workers, despite some literature showing that paramedics (albeit in Canada) do not feel equipped to address such issues. A small number of examples of where paramedics are addressing the social and psychological needs of patients and families in addition to their medical role has been seen in Northern Queensland, India, and Ghana. Paramedics have provided public health and social provisions as well as dietary, sexual, intimate partner violence, and general social health advice (Ashtekar and Mankad 2007; Reeve et al. 2008); however, there is no information specifically about children and young people. Despite this, past research has suggested that in certain countries, student and qualified paramedics consider their main role to be that of transportation and treatment of immediately life-threatening conditions only, and do not consider psychosociology to be an important aspect of practice (Roberts and Henderson 2009). Jones et al. (2010) highlights that pre-registrant student paramedics felt that psychosociology had "little relevance" to their practice, and failed to "relate it to the job at all". Students showed a hierarchy of knowledge that not only places more emphasis on practical hands-on paramedicine, but which is at odds with the profession's own view of what should constitute the nature of 
modern paramedic practice. Such views and attitudes towards psychosociology are disconcerting, especially given that the WHO (2011) estimated that around $20 \%$ of the world's children and adolescents have mental disorders or problems (often caused or exacerbated by social problems). Moreover, there is overwhelming evidence to suggest that child neglect and abuse can result in multiple lifelong psychosocial health problems.

While there appears to be some evidence of understanding how paramedics prioritise knowledge in their practice, there is a distinct lack of research specifically about children and young people, which is needed to inform future practice, policy, and education. Campbell and Rasmussen's (2012) ethnographic research asserts that paramedics do not provide for psychosocial needs or provide follow-up care; however, it is known that UK paramedics often encounter these patient groups. Identified challenges include a high number of calls with psychosocial needs, overworked paramedics, large numbers of nonmedical calls, and a general lack of resources for patients with nonmedical needs. Such research is not only dated but also is from a Canadian perspective and thus is limited somewhat with regard to UK paramedic practice. No similar UK-based study was identified in this review. Without investigating how UK paramedics perceive their roles in practice and their knowledge of psychosocial determinants of health when encountering children and young people, the extent to which they will be able to promote good health practice remains unknown. Furthermore, the ability levels of paramedics to recognise and refer potentially damaging psychosocial factors remains unknown, and thus their efficacy cannot be truly assessed. More research is required to inform future paramedic practice, education, and policy, and to advertise the key role paramedics play in child and family health. 


\section{Theoretical perspectives: Carper's ways of knowing}

The transition from protocol-driven training to evidence-based multidisciplinary education represents a shift in the way modern-day paramedics are taught and encouraged to think, and thus represents a shift in what paramedics know. Paramedics are now educated to critically examine the evidence to justify a course of action or inaction in their patient care which, in turn, represents a shift in the types of actions carried out by paramedics. Some of these actions include complex ethical and moral decisions and are arguably influenced not only by the education and training paramedics receive, but also by what they have come to know through their own practice and their own life experiences. This term 'knowing' refers to ways of perceiving and understanding the self and the world (Chinn and Kramer, 2011), and what is known by paramedics underpins their practice and defines their profession. Chinn and Kramer (2011) state that in a discipline, knowledge represents what is collectively taken to be a reasonable and accurate understanding of the world as the members of the discipline understand it. The 'knowledge' of the discipline is that which has been collectively judged by standards and criteria shared by members of the disciplinary community.

The in-depth review of the literature has thus far identified a lack of consensus surrounding what is collectively taken to be a reasonable and accurate understanding of child protection. Furthermore, it has highlighted a lack of standards and criteria as applied to paramedics, as well as any literature investigating the collective understanding of these criteria. In effect, little is known about what paramedics themselves know. Understanding more about what paramedics know about CP and how they come to know what they know is of interest to this research. Understanding how this knowledge is used in practice to reach decisions about children and young people, and how this knowledge influences paramedic perceptions of their role in this process will help inform future paramedic practice, policy, and education.

Knowing is an ontological, dynamic, and changing process (Chinn and Kramer, 2011) and differs depending on the context in question. In 1978, Barbara Carper published her theory 
of the 'four ways of knowing' in nursing. This epistemological theory has become widely read, discussed, and incorporated into the theoretical underpinnings of nursing education, research, and reflection (Rubarth, 2005; Clements and Averill, 2006; Guadron, 2008). Carper (1978) suggests the four ways of knowing are empirical, aesthetic, personal, and ethical. Clements and Averill (2006) state that when Carper's conceptual framework is applied in an integrated manner, it can provide a holistic way of deepening the understanding of nursing practice and of the nurse as an individual. This theory can equally be applied to paramedics, although no literature has been identified where it has been used. Given such similarities, Carper's conceptual framework can be applied to paramedicine also.

Carper explains that nurses' knowledge has patterns and forms structure to justify decisions, actions, and inactions and that understanding these structures is essential to the teaching and learning of practice. Guadron (2008) investigated the efficacy of a way of knowing scale, derived from Carper's original theory. The scale indicated that community nurses with more years of education use more integrated ways of knowing, as opposed to favouring only one or two. This new data could then help to better understand this professional group and to design educational updates and interventions. Applying Carper's ways of knowing to research with paramedics about child protection can allow similar learning to take place. If it is possible also to measure which type of knowledge paramedics use the most, it might also be possible to focus the delivery of educational interventions more specifically, thus informing practice. Chinn and Kramer (2011) state that since Nightingale first established formal secular education for nurses, nursing has depended on formal knowledge as a basis for practice. While the nature of knowledge changes with time, the overall fundamental values that guide nursing practice have remained remarkably stable. There are similarities between modern paramedicine and nursing, such as higher education, physical skills training, evidence-based practice, the use of guidelines, and professional registration. Furthermore, nurses themselves are equally responsible as paramedics under section 11 of the Children Act 2004. Each 'way of knowing' will now be explored. 


\section{Empirical knowledge}

Empirical knowing is defined as the 'science' of a discipline (Clements and Averill 2006) and refers to information that is factual, objective, descriptive, evidence-based, and therefore verifiable (Rubarth 2005). Carper (1978) defines it as knowledge gained through systematic investigation, which assumes that what is known is accessible through the senses (Chinn and Kramer, 2011). Empirical knowledge is taught knowledge through evidence-based educational programmes and is embedded within the positivist paradigm. Empirical knowing is a school of thought upon which much emphasis is placed when developing a scientific body of healthcare knowledge (Berragan, 1998). It is helpful to use Rubarth's rubric, supported by various authors, to critically question Carper's four ways of knowing, beginning with empirical knowledge. The critical question must be "What is it and how does it work?" (Rubarth, 2005). In the case of paramedics, patients can be assessed based on physical signs and symptoms, definitively proved causal factors, and objective information gleaned from monitors and basic blood tests. Treatment can be provided based on evidence drawn from results from randomised control trials and known pharmaceutical interactions. The paramedics' knowledge of evidence-based practice and research influences their assessment, and an empirical meaning of a situation is formed.

\section{Aesthetic knowledge}

The aesthetic way of knowing is often non-verbal and is shared by the practitioner and the client through a perception of what is meaningful in each situation (Clement and Averill, 2006). Aesthetics is a noun that derives from the Latin and Greek words referring to perception. It has evolved to refer specifically to the perceptual ability to appreciate artistically valid forms. The adjective 'aesthetic' identifies an object or experience as aesthetically valid, but does not necessarily equate to that which is viewed as beautiful of lovely (Chinn and Kramer, 2011). Carper (1978) defines aesthetic knowledge as being subjective, a view congruent with that of Berragan (1998), who describes it as being linked to the interpretive/constructive paradigm. Aesthetic knowing is expressed through the actions, bearings, conduct, attitudes, individual narratives, and interactions of nurses with others (Clement and Averill, 2006), and is often called the 'art of nursing'. This type of 
knowledge comprises perception, understanding, and empathy, and acknowledges the value of everyday experiences lived by individuals and the role of intuition through experience in practice (Berragan, 1998) and of knowledge which is built up over the years in practice. Guadron (2008) states that this art incorporates clients' perceptions, meanings, values, and preferences in decision-making, and to understand this is to understand the person and thus the patient-practitioner relationship. Using Rubarth's rubric, the critical question for the aesthetic way of knowing is "What does this mean?" (Rubarth, 2005). In assessing patients and situations, the paramedic "comes to know" that the patient is upset, and is at risk, or a victim of abuse or neglect, by incorporating the individual's lived experience and health narrative, and their own meaning of health into the assessment. This art is heavily influenced by previous experiences, and the understanding of relationships, human interactions, and reflections throughout years of practice. Similarly, aesthetic knowledge can also mean "to envision what is possible but not yet real", or imagining what could happen if something were not done (Chinn and Kramer, 1999, p. 10); again, this takes account of previous experiences.

\section{Ethical knowledge}

Ethical knowing focuses on the moral or ethical component of practice. Paramedics make decisions based on what is right or good for the patient, and thus, ethical knowing involves making moral judgements and right decisions at the right moment. Ethical knowing involves focusing on what ought to be done in a situation, offering alternatives, and being responsible for the safety, best interests of the patient and family, and for upholding their values (Rubarth, 2005). The HCPC (2016) standards of conduct, performance, and ethics gives direct guidance on ethics for paramedic registrants and can be used to inform ethical knowing. Clements and Averil (2006) assert, however, that practitioners may not even be conscious that they are making ethical decisions throughout an entire day or shift, and that many of these decisions present as a common sense based decisions. This assertion presents a challenge to those attempting to research such knowing, as respondents may not be able to estimate its use. Ethical decisions involve upholding the beliefs and values held by 
individuals, groups, and societies. More specifically, Carper (1978) states that ethical knowledge or knowledge of morality goes beyond simply knowing the norms of ethical codes of disciplines, and includes all voluntary actions that are deliberate and subject to the judgement of right and wrong. The act of a paramedic caring for someone is, for example, a deliberate action or series of actions set in place to accomplish defined goals. Such actions and goals will inevitably involve the need for ethical thinking and the knowledge of moral obligations, moral and non-moral values, and desired ends (Fawcett et al., 2001). Using Rubarth's rubric here, the critical questions for the ethical way of knowing for the paramedic are "Is this right? Is this responsible?" (Rubarth, 2005), or similarly, is not doing something right or responsible - in the case, perhaps, of reporting or investigating concerns of suspected child abuse or neglect.

\section{Personal knowledge}

Personal knowing refers to knowledge of the self; encountering and actualizing of the concrete individual self; and development of an interpersonal relationship between the practitioner and patient (Carper, 1978). Clement and Averill (2006) state that personal knowledge involves a measure of self-knowledge, confidence, self-responsibility, and engagement with life. They go on to state that it is expressed as a state of being, a personal experience reflecting inner and outer harmony, "knowing that and knowing how" (Clement and Averill, 2006 p271). Berragan (1998) suggests that for these reasons, it is perhaps the most difficult pattern of knowledge to evaluate; again, it is constructionist interpretivist in nature, and that it is of extreme importance to all areas of practice, without which the development and improvement of individual and group practice would be impossible. Personal knowing involves openness to experiencing the world with others and having meaningful mutual interactions with others, as happens in clinical practice. Personal knowing involves knowing what affects you as an individual and how this, in turn, affects a relationship. Using Rubarth's rubric, the critical questions for the personal way of knowing are "Do I know what I do?" and "Do I do what I know?" (Rubarth, 2005). This way of knowing can be a method by which paramedics ask themselves what they know about the case of suspected child abuse, for example. The more comfortable the paramedic is with 
their own knowledge and abilities, the more comfortable the paramedic will be with their assessment, skills, and evaluation. Carper (1978) posits that personal knowing requires maturity, self-confidence, and trust in oneself, and emerges more completely throughout life, leading one to surmise that personal knowledge might increase with age and clinical experience.

Carper's theory provides a foundation for the measurement and assessment of ways of knowing, and it has been used by various authors to explore nursing practice (Rubarth, 2005; Payley et al., 2007; Carnago and Mast, 2015). There is a lack of paramedic-based research using this theory to explore how actions, inactions, and decisions are made. However, there is potential for its use within this thesis. Various methods of investigating decision making by paramedics have been used by multiple authors and tend to focus on intuitive and experiential processes. Carper's theory is more inclusive; it critically questions how clinicians come to know what they know, and more specifically, details and investigates this knowledge. Carnago and Mast (2015) posit that using multiple ways of knowing strengthens the clinician-patient relationship and increases the likelihood that patients will receive ethical, individualised, and effective care. This assertion validates an attempt to determine if, and to what extent, different ways of knowing are used in paramedic practice. This data can be used to inform future practice and education. When multiple ways of knowing are intentionally used, clinicians can strengthen their ability to assess needs, relate effectively with marginalised patients, and intervene more effectively in complex cases (Carnago and Mast, 2015). Understanding more about to what extent these ways of knowing are used in paramedic practice can assist in understanding paramedic perceptions of their role in protecting children and young people. Rubarth (2005) states that Carper's conceptual framework is used to study nurses' abilities systematically, and each specific 'way of knowing' may potentially play a role in the nurses' approach to various tasks. Guadron (2008) goes further and states that while nurses use different ways of knowing in their decision-making, each 'way' is necessary for the development of mastery in nursing, with no single way of knowing being used in isolation, as this would not be sufficient. Thus, a study of such 'knowing' may inform individual and professional practice, policy, and 
education, and if applied to paramedic CP practice, can be used to achieve the aims of this thesis.

Carper's ways of knowing can be used as a framework for reflection and has been incorporated into reflective models such as Johns (1995) which has been promoted to paramedics as a valid guide to reflecting on their clinical practice (Turner 2015) and reflecting upon how they come to make decisions (Paramedic CPD, 2013). Empirical knowing for paramedics could be formed from their university education or their continual professional development for example. Aesthetic knowing could be formed from their memory of previous experiences and how they dealt with them or how they have reflected upon mistakes and errors made in practice and this has come to make them feel. As a researcher, asking "how do paramedics make decisions" is a very broad and non-specific approach. Moreover, framing the results from the systematic review of the literature, questionnaire, and focus groups could be difficult without a focused approach. I chose Carper because her theory provides an easy to use framework for analysing the results in a more focused manner and allows a much broader question to be asked in a way that separates key tenets of decision making and aides the discussion process.

There are however limitations to Carper's theoretical framework; not least that it was developed in 1978 and has since remained relatively unchanged. It has been argued that Carper's way of knowing is a valid foundation for reflection but that it limits the use of other equally valid patterns of knowing and does not adapt to the changes in modern practice. Chinn and Kramer (2008) added emancipatory knowing for example, which involves the critical analysis of the status quo of practice and how change can be informed. It is thought that introducing an emancipatory way of knowing can take many years and is best used by practice leaders only, which limits its applicability and usability in research but does show how Carper's theory can be expanded. Similarly, Schultz and Meleis (1998) identified the need to add practical knowing and White (1995) discussed the need to add a socio-political pattern of knowing. These additions predominantly refer to the nursing profession which is 
more established than paramedicine but does evidence that Carper's original framework is limited when compared to modern practice. In a similar vein, the increasing emphasis placed on evidence-based clinical practice limits patterns of knowing such as personal and aesthetic knowing; and thus, Carper's entire framework. Empirical knowing appears to dominate modern practice to the deficit of other areas that are considered equally as important to the ethos of nursing; such as ethical knowing (Porter, 2010). This hierarchy, while counterproductive, limits the use of Carper's original framework for many types of research. Heath (1998) discusses how experience can influence nursing practice and the expertise of that practice and notes that limited experience can restrict the use of frameworks; such as Carper. To build both personal and aesthetic knowing a practitioner needs to gain experience and needs to have time to reflect upon their experience consciously or unconsciously for it to influence their decision making.

While the degree to which Carper's framework represents paramedic knowledge in $21^{\text {st }}$ century practice must be questioned, the simplicity of the framework itself was an advantage for this research, and it is notable from the literature that this framework continues to be applied within the professions of nursing and medicine (Carnago and Mast 2015).

Although other theoretical frameworks were considered, such as Benner's (1984) 'Novice to Expert' model, Shuler and Davis's (1993) nurse practitioner framework, and Kramer and Chinn's (1988) 'Perspectives of Knowing' framework. Many of these frameworks were however very specific to nursing practice and introduced more patterns of knowing specific to different stages of nursing practice; such as advanced and expert practice. In my opinion, Carper's framework better represented the infancy of paramedicine as a research-based profession provided a simple approach to a wider and more complicated question of how paramedics come to make decisions in child protection. It gave me a coherent structure upon which to plan and organise my research as well as to show and explain how changes could be made to future education and training. 


\section{Literature review conclusion}

This review of the literature has highlighted the key role paramedics play in child protection and the unique position they hold to experience and witness things that other health and social care professionals cannot. For many people, the word 'paramedic' perhaps conjures up concepts of speeding ambulances in dramatic and often traumatic scenarios where time is limited and lives are often lost. This view, however, is a limited one, and suggests that the public misunderstands the role of paramedics - although like so much of paramedic practice, there is no research which has directly investigated this. The role of paramedics in the UK now involves far more than the stabilisation of life-threatened patients through illness or injury, and instead consists of a multitude of tasks shared by other professions who work within the community. Paramedics have a role to play in chronic illness management, disease prevention, health promotion, and child protection.

This review has identified that paramedics are equally accountable for their actions to protect children and young people under various pieces of legislation as other HCPs. However, unlike for other HCPs, little research exists which investigates these actions. It has been observed that there have been no empirical studies undertaken in the UK in the last 20 years which specifically address the level of knowledge paramedics have in child protection. Furthermore, there have been no studies undertaken to investigate the level of confidence paramedics have in carrying out their role in child protection, or their perception of this role. There appears to be some evidence to suggest limitations in paramedic knowledge of subjects such as child sexual exploitation, witnessed domestic violence, parental/carer mental health problems, substance misuse, and youth homelessness, but there remains little in the way of child protection as a distinct subject area. Without this research forming a valid evidence base, current practice, policy, and education cannot be fully informed. There is a large amount of child protection literature which mentions multidisciplinary and community work as well as the need to collaborate, identify, intervene, and educate. However, despite all of this, little mention is made of paramedics and their key role in child protection. This review has drawn from a range of different evidence bases to show the 
multiple roles paramedics can play in protecting children and young people, and to identify how paramedicine remains an under-researched area of practice particularly in relation to child protection. In order to fully inform future paramedic practice, policy, and education, more research is needed to investigate the roles of paramedics in child protection. Similarly, more research is needed to investigate how confident paramedics feel in carrying out this role, and what knowledge bases they use to do so. While child protection is everyone's responsibility, without further research, it remains unknown to what extent paramedics are able to fulfil their responsibility.

\section{Formulating research questions}

This review of the literature has highlighted the key role paramedics play in child protection. However, several outstanding questions remain, for example, questions surrounding how paramedics themselves perceive their role, how they are trained to undertake their role, and how confident they are in this role. The review of the literature has provided a muchneeded context to the role of paramedics, but has fundamentally highlighted the paucity of research in this field, which may be linked to the infancy of the paramedic profession or a misunderstanding of the profession.

To fulfil the primary aim of informing future paramedic practice, education, and policy, and now with a better understanding of the gaps in the current research literature, this chapter aims to outline the study's specific research questions. It was deemed prudent to remind the reader of the research objectives.

The specific objectives are as follows:

- to examine the perceptions paramedics' have of their role in child protection

- to examine how paramedics' perceive the training they receive in child protection

- to examine how confident paramedics are in child protection

- to identify any barriers to paramedics undertaking their role in child protection

- to identify ways of improving the paramedic's practice in child protection 
Specific research questions needed to be formulated to achieve these objectives. To achieve the aims of this study, the research questions needed to be formulated from the analysis of the existing literature and the identified gaps in research. These questions needed to be concise, specific, and focused on their ability to provide answers. Bryman (2008) states that poorly researched or poorly constructed questions will inevitably lead to poor research. Furthermore, if the researcher does not specify clear research questions, the data collection will be confusing, the research itself will be unfocused, and the results will be less useful, irrespective of the amount of financial investment or researcher experience. Thabane et al. (2008) similarly state that the success of any research process relies on how well the investigators can translate a problem (a lack of data for example) into a research question. The review of the literature has highlighted the gaps in what is known about the way paramedics perceive their role, how confident they are in their role, and how they are educated. With these gaps identified, the author used the Patient, population problem, Intervention or exposure, Comparison, and Outcome (PICO) approach to aid in formulating specific questions. PICO was originally developed around evidence-based medicine and was designed for clinical studies. However, multiple authors have advocated its use in framing research questions in different areas, including nursing, palliative medicine, occupational health, clinical epidemiology, and systematic reviews (Springett and Campbell, 2006; Thabane et al., 2008). Focused research questions have multiple advantages, which include easing and guiding data collection and the analysis and writing up of data, as well as helping to prevent the author from going off on a tangent and in unnecessary directions (Bryman, 2008). Guided by the PICO framework, this researcher compiled specific research questions. The answers to these questions should achieve the objectives outlined earlier within this section and achieve the overall aim of this study, which is to inform future paramedic practice, education, and policy. 


\section{Research Questions}

1. How do paramedics perceive their role in child protection?

2. How do paramedics perceive the training they receive on child protection?

3. How confident are paramedics in child protection?

4. What barriers exist to paramedics undertaking their role in child protection?

5. Can any ways of improving paramedic's practice in child protection be identified? To provide the answers to these clearly defined research questions, a method of data collection was needed. The next section introduces the methodology through which the theoretical underpinnings of Carper will be integrated. 


\section{Chapter Three: Methodology}

\section{Nature of enquiry}

Ontology is a term which derives from the Greek words 'onto' meaning 'being' and 'logos' usually interpreted as 'science'. Ontology has been traditionally understood as the science or study of being (Lawson 2004). Mosses and Knutsen (2007) state that ontology relates to the nature and construction of social reality and considers what the world is made up of: the theory of existence and being. The main competing ontological perspectives in social research are objectivism and constructionism (Soini et al., 2011). The objectivist ontology views the social world as having fixed properties and relations that exist independently of the researcher's consciousness and that of others inhabiting it (Crotty, 2012; Schuh and Barab, 2008), and is associated with quantitative research. A constructionist ontology, however, views the world as a socially constructed one, into which we are already born and in which meaning has already been made. This social construct is seen to be affected by those inhabiting it (Crotty, 2012; Scotland, 2012) and lends itself to qualitative research.

While the research ontology seeks to explore how the social world is constructed, the epistemology explores the theory of knowledge and what it is possible for the researcher to know through different epistemological perspectives, such as positivism and interpretivism. Epistemology looks at the origins of nature and the limits of human knowledge (Schuh and Barab, 2008). One could argue that ontology and epistemology can be mutually supportive in understanding a piece of research, with the ontology asking what there is to know, while the epistemology asks how we can come to know it. A positivist epistemology supports an objectivist approach and assumes that there are objective facts in the world separate from consciousness and where the researcher and researched are independent entities. This knowledge is obtained through impartial, rigorous, quantitative, and objectively tested scientific principles, laws, and experiments (Crotty, 2012; Scotland, 2012). In contrast, however, an interpretivist epistemology supports a constructionist approach and is one of subjectivism. This approach is based on real-world phenomena in which reality does not 
exist independently of its inhabitants' knowledge of it; it allows for different people to construct meaning in different ways, from different perspectives and explores the topic in a qualitative manner (Crotty, 2012; Scotland, 2012). This ontology surmises that meaning is dependent on individual participant's experience, and that the researcher investigating this experience can affect meanings through his or her interpretation. This can occur when undertaking, guiding, and thematically analysing focus groups and can often be of benefit as well as a source of researcher bias.

Having reviewed the literature and reflected upon the aims of this research, one could argue that social science research can be underpinned by both different schools of thought. Quantitative findings can be ratified by qualitative findings and vice versa. Independent variables, such as demographic factors, are objectivist positivist in nature, while the respondents' perceptions of their role and confidence are constructionist interpretivist in nature. The age of a participant may objectively affect the way in which a decision is made, which can best be explained through the understanding of the respondent's own meaning of age in context. This argument, however, is not supported by all researchers. Guba and Lincoln (1994) posit that quantitative and qualitative methods are each inextricably tied into specific sets of ontological and epistemological assumptions and that it is, therefore, impossible to combine the two approaches for paradigm stances, a view which has not gone unchallenged by many authors (Soini et al., 2011). The dichotomously opposing views of objectivist positivism and constructionist interpretivism are arguably aligned under Carper's (1978) seminal theory of 'ways of knowing'. Carper argues that multiple ways of knowing are used simultaneously (some subconsciously some not) to come to a decision, and that different ways of knowing form structures in individual experiences to justify decisions, actions, and inactions. This theory has been viewed as an epistemological underpinning in various pieces of research (Rubarth, 2005). Empirical knowledge follows the objectivist positivist nature of enquiry, where knowledge is usually quantifiable and objective, such as clinical observations. Aesthetic knowledge, however, is largely constructionist interpretivist in nature and relies on what the person has come to know and what meaning has been placed on the context of knowledge. Carper argues that these two lines of thought come 
together as one to influence how a decision is made. Rubarth (2005) states that Carper's conceptual framework can be used to study nurses' abilities and decision-making processes. Moreover, each specific 'way of knowing' may potentially play a role in the nurses' approach to various tasks, and understanding the role of knowing can inform educational approaches and highlight deficiencies in certain elements of practice. This nursing-based research can be adapted to paramedic practice

How knowledge develops is an epistemological concern that can reveal how paramedics come to know, and how they acquire shared knowledge in the discipline. Knowing is an ontological, dynamic, and a changing process, where knowledge refers to knowing that is expressed in a form that can be shared or communicated with others, and thus learned from. Although there are differing views on how two opposing schools of epistemological thought can be used in one research process, Carper's theory argues that practitioners use different ways of knowing in their decision-making. Each 'way' is necessary for the development of mastery in that practice area, with no single way of knowing being used in isolation, as this would not be sufficient (Guadron, 2008). This approach to social science research lends itself to an explanatory sequential research design, which will be explored in the next section.

\section{Design: Sequential design}

Sequential research is that which is carried out using a staged approach. Its aim is for each previous stage to inform the design or approach of the next. An explanatory sequential research design involves the investigator collecting quantitative data followed by qualitative data, which helps to explain or elaborate upon the quantitative results (Byrne and Humble, 2007). This mixed-methods sequential explanatory design is highly popular among researchers, and its characteristics are well described in the literature. This method has found application in both social and behavioural science research (Ivankova et al., 2006) and is the underpinning methodology of this thesis. The review of the literature has highlighted gaps in the research; these gaps have influenced the construction of the research questions, 
and these will be explored quantitatively first, and then later explored and elaborated upon qualitatively. It was envisaged that such an approach would facilitate an in-depth exploration of the topic at hand from various methodological angles, validating any conclusions drawn from the data.

As with any research method, there are weaknesses to this design. Sequential explanatory research is very time-consuming given the various stages of the research, which in turn, can also increase costs. No stage can continue until the previous one has been sufficiently analysed, and so delays in the sequence can sometimes occur. Furthermore, there are limitations when the same group of individuals are not used for both quantitative and qualitative stages, although it is recognised that larger samples are sometimes easier to gain when using online-based quantitative methods. As such, often only some of the respondents will feature in both stages. The strengths of this design, however, include its simplicity. The separate phases make this design straightforward to describe, implement, and report, which was considered an advantage since PhDs can often take between 3-6 years. Although this design typically emphasises the qualitative aspect, the inclusion of a quantitative component can make the qualitative approach more acceptable to quantitative-biased audiences, thus, possibly increasing the impact of any findings and conclusions (Ivankova et al., 2006).

This thesis is presented sequentially. The methodology section outlines the methods used in the sequence in which they were undertaken. Similarly, the sample/participants, measurements, and procedure sections are all presented in the sequence in which they were used or gathered. By presenting them in this way, it is thought that the reader will be better able to understand the research journey that was undertaken, and better able to understand how each element of this research was informed. The findings and discussion chapters are presented in a more integrated way. This approach allows for a more structured and cohesive analysis of the data, which is presented more coherently. 


\section{Mixed methodology}

An explanatory sequential design uses mixed methods to achieve the overall research aims. Often referred to as the third methodological movement, the use of mixed methods research has seen a rapid rise in popularity within the last ten years (Cameron, 2011). Mixed methods research is defined by the Journal of Mixed Methods Research (2018) as "research in which the investigator uses both qualitative and quantitative approaches or methods in a single study or program of inquiry". The rationale behind using mixed methods was born from its ability to transcend multiple theoretical paradigms. Mixed methods processes can lead to increased validity, the creation of new lines of thinking through the emergence of new perspectives, and the development of research through one approach informing the other (Anderson, 2006). The review of the literature gave a basis upon which research questions could be asked and measured quantitatively before being explored in more depth through qualitative exploration.

Some of the challenges and limitations of mixed methodological research include having to overcome philosophical differences and having to be proficient and competent in multiple methods. By having to do this, the researcher often has to invest significantly more time in data collection and analysis than if one single method were used (Anderson, 2006; Cameron, 2011). Despite this, however, Venkatesh et al. (2013) argue that mixed methodological approaches are considered a major strength and of relative value in the research of social and behavioural sciences. Furthermore, Giddings (2006) suggests that such methods extend the repertoire of social science and health research, and that more certainty can be gained from mixed methodological results, given their ability to be holistic, comprehensively well-rounded, and inclusive of multiple approaches. This approach arguably provides validity, reliability, and applicability. These individual constituents will now be explored further.

\section{Quantitative design}

Quantitative research is described by Muijs (2010) as the process by which phenomena can be explained through the collection of numerical data that can be analysed using 
mathematically based methods, such as frequencies. Bryman (2008) expands upon this explanation by describing quantitative research as not only the collection of numerical data, but as an exhibition of a relationship between theory and research using a natural scientific approach, and as having an objectivist conception of social reality. Key characteristics of quantitative research include its ability to remain objective, unbiased, and itself measurable against statistical tests, and it tends to be associated with large-scale analysis which investigates causal hypothesis (Anderson, 2006). The rationale behind using a quantitative methodology lies in its ability to identify significant findings clearly. Furthermore, in addition to such findings going some way to answering the research questions, they will also guide the design of the qualitative element of this thesis. The methods used to analyse this quantitative data were also mixed, and include descriptive statistical analysis and independent sample t-tests.

\section{Descriptive statistical analysis}

The descriptive statistical analysis is used to describe the basic features of the data collected, that is, what is and what is not. Descriptive statistics, when used alone, cannot robustly demonstrate causal analysis; however, they can be used to present data in a coherent manner which permits main characteristics to be identified. This often allows further exploration to take place and can inform the design of the subsequent research (Marshall and Jonker, 2010). This is the first type of research to look solely at the role of paramedics in child protection. This researcher was experienced in working qualitatively, but found more the quantitative statistical methods highly challenging. Thus, the decision was made to begin with descriptive statistics only. This allowed the researcher to progress with the overall aims of the research, but also provided a foundation upon which further analysis could be undertaken later. Despite the limitations to using descriptive statistical analysis alone, it is still viewed as a valid and reliable method which can add otherwise unknown knowledge to a professional and academic field (Marshall and Jonker, 2010). This approach is not entirely unusual when undertaking mixed methods research with an explanatory sequential design. Mixed methods designs can provide pragmatic advantages when exploring complex research questions. The qualitative data provide a deep 
understanding of survey responses, and statistical analysis can provide a detailed assessment of patterns of responses. However, the analytic process of combining quantitative and qualitative data can be time-consuming and expensive and thus may lead researchers to reduce sample sizes or limit the time spent in focus groups. Ultimately, if the researcher does not want to reduce the sample size or the focus group time, then the most appropriate mixed method design will often be one that does not require extensive multivariate analysis of quantitative data (Driscoll et al., 2007). While descriptive statistics are solely concerned with properties of the observed data, other forms of statistics, such as inferential statistics, can infer meaning, and will not solely describe the properties about a specific population. The next section explores this.

\section{Independent sample t-test}

Given the varied differences of paramedics currently employed within UK ambulance services, it was considered important to compare the answers provided by the different groups in order to inform future paramedic practice, education, and policy better. For example, as statistically significant differences in responses were found between male respondents and female respondents, the possible causes of these differences had to be investigated and these in turn could themselves inform future research, educational programmes, and training updates. To do this, the researcher undertook independent sample t-tests. An independent sample t-test compares the means of two independent groups to determine whether there is statistical evidence that the associated population means are significantly different. The researcher used the Statistical Package for the Social Sciences (SPSS) computer software to undertake this analysis.

There is a long-standing debate over whether Likert scales produce ordinal or interval level data (Parker et al. 2002). The answer reached determines the choice of statistical technique. Typically, if it is thought that the scales yield interval data then, subject to other conditions such as normality of distribution being met, parametric methods are applied. If it is thought the scale produces ordinal data, then non-paramedic methods are seen as more appropriate. Wu and Leung (2017) state that, strictly speaking, Likert scales produce ordinal 
data as the scale's numerical markers represent subjective descriptors that are not equally distant from one another. The use of numbers can create the illusion of equidistance between points. They note though that viewing the data as interval data is common and appropriate in many cases. Many others also clearly view Likert scale data as interval data and argue that analysis on this basis can lead to fruitful, meaningful and valid findings (see Knapp, 1990, Grolnick et al. 1997, Harwell and Gatti 2001).

There is recognition from both sides of the debate that if parametric techniques are to be applied a large sample size is preferable. The sample size in this research was large and influenced the decision to use a parametric technique: the independent sample t-test. For comparison and peace of mind, non-parametric tests were also run. They generated similar outcomes, with no substantive difference. Caution has also been applied in interpretation, the findings have been considered within the context of descriptive data and focus group discussions.

When comparing both respondents' length of service and years of qualification to their questionnaire responses the groups were split into 0-14 years and $14+$ years. This decision resulted from an error in the data collection where the demographic survey questions did not correctly separate the measures. For example, respondents were asked "About how long has your length of service been?" and given the possible responses of 0-2 Years, 2-4 Years, 4-6 Years, 6-8 Years, 8-10 Years, 10-12 Years, 12-14 years, 14 Years or More. This meant that it could not be accurately determined to which point respondents were referring when relating to the higher number of years in each section. If they had served 6 years for example which answer would they have provided 4-6 years or 6-8 years. This error meant that the only two separate groups that could be measured were $0-14$ years and $14+$ years; although this is also not without its limitations.

\section{Qualitative design}

The term 'qualitative research' is used not only to refer to the techniques of data collection and analysis, but is also used to refer to the wider framework for conducting the research itself (Braun and Clarke, 2013). Bryman (2008) describes qualitative research as a strategy 
which emphasises words rather than the quantification of collected data. This method allows researchers to capture and record participants' experiences and gain richer and more complete descriptions and understandings of events (Stuckey, 2013). Qualitative research has the potential to go much further than merely reporting people's views and opinions. It encompasses a variety of ways to explore human behaviours, actions, and experiences and provides opportunities to understand what people are doing and why (Williams, 2012). Qualitative research is an umbrella term which encompasses a wide range of methods, such as interviews, focus groups, and case studies. It is through these methods that a deeper understanding of participants' narratives and meanings are explored within a specific context. The advantages of qualitative methods are numerous and are often explained in contrast to quantitative methods. The use of open-ended probing questions, for example, gives participants the opportunity to respond in their own words, rather than forcing them to choose from fixed responses, as is the case with quantitative methods. Group dynamics facilitated through qualitative methods often evoke responses which are meaningful and culturally salient to the participant or group and are often unanticipated by the researcher (Mack et al., 2005). The flexibility of qualitative research is also considered an advantage. A researcher who is unfamiliar with a specific context can view it from an outside perspective and ask questions which the individual or group may not have asked themselves. This can lead to a deeper understanding of the topic in question and can give rise to more or different questions. Nonetheless, qualitative research is not without its disadvantages.

The inherent limitation of the qualitative research is that its findings cannot be extended to wider populations with the same degree of certainty that quantitative analyses can. This is because the findings of the research are not tested to discover whether they are statistically significant or due to chance (Atieno, 2009). It could be argued that the beauty of qualitative research is found within the fact that the findings are more personal and individual and so its limitation in comparison with quantitative methods is actually what makes it so valid. Various other disadvantages exist, such as the possibility of research bias and researcher misunderstanding. Use of language, colloquial dialects, and idiolects all have the inherent 
possibility of misunderstanding, misinterpretation, and misrepresentation, which is less possible with the closed questioning of quantitative data. Nevertheless, qualitative research facilitates our understanding of human experience, and helps us learn more about human motivation, perceptions, and behaviours (Williams, 2012) and has a place in paramedic related research. One could not fully explore the meanings, understandings, perceptions, and confidence levels of paramedics without the use of qualitative methods. The analysis of the qualitative data was thematic in nature, as will be discussed in this following section.

\section{Thematic analysis}

Thematic analysis is the process of Identifying, analysing, and reporting patterns (themes) within data. It organises and describes a dataset in rich detail and interprets various aspects of the research topic (Braun and Clarke, 2006). Holloway and Wheeler (2009) state that a theme is a cluster of linked categories conveying similar meanings and forming a unit or a theme. Such themes derive directly from the data through the identification of concepts, categories, and recurring regularities, which the researcher later transforms through analysis. Thematic analysis is considered appropriate for any study that seeks to discover through interpretation. It provides a systematic element to data analysis and allows the researcher to demonstrate an association between a group of people and a theme, and so answer the research questions. Braun and Clarke $(2006$, p. 35) devised a framework, which this research followed, to ensure a valid and rigorous approach to the analysis, as can be seen below.

1) Familiarising yourself with your data: Transcribing data (if necessary), reading and rereading the data, and noting down initial ideas.

2) Generating initial codes: Coding interesting features of the data in a systematic fashion across the entire data set, and collating data relevant to each code.

3) Searching for themes: Collating codes into potential themes, and gathering all data relevant to each potential theme. 
4) Reviewing themes: Checking if the themes work in relation to the coded extracts (Level 1) and the entire data set (Level 2), and generating a thematic 'map' of the analysis.

5) Defining and naming themes - coding: Ongoing analysis to refine the specifics of each theme, and the overall story the analysis tells; generating clear definitions and names for each theme.

6) Producing the report: The final opportunity for analysis. Selection of vivid, compelling extract examples, the final analysis of selected extracts, relating back to the analysis of the research question and literature, producing a scholarly report of the analysis.

(Braun and Clarke, 2006, page 35).

The term 'coding' refers to a marker applied to a section of raw data, such as text within a transcript. Saldana (2012) describes a code in qualitative inquiry as a word or short phrase that symbolically assigns a summative, salient, essence-capturing, and evocative attribute for a portion of language-based or visual data. These codes are applied or linked to raw data as summary markers for later analysis, which may include comparing the relative frequencies of themes or topics within a data set (Namey et al. 2008). In addition to the manual coding of the data set (with the use of codes and colours), the transcribed data were uploaded into thematic analysis software NVIVO to assist in further analysis. NVIVO is described as software that supports qualitative and mixed method research by organising unstructured or qualitative data (QSR International 2017). Hilal and Alabri (2013) suggest that the use of NVIVO in qualitative research is well established and boasts several advantages, such as increased time efficiency, the ability to support findings more rigorously, and being able to work more methodically through large data sets. Following Braun and Clarke's framework, the researcher undertook the thematic analysis and used NVIVO to affirm the themes found and to identify any themes not identified by hand. No further themes were highlighted using NVIVO after manual coding and analysis, which itself 
is not a disadvantage, but rather supports the assertion that the all the pertinent themes had been highlighted.

This thematic analysis broadly follows Braun and Clarkes' framework, above, however, as a novice researcher it was considered useful to set out how each step of this process was undertaken:

- During focus groups, rough notes were made of salient points made, examples of which can be seen in Appendix 2. These notes also included reference to any body language which was found notable; i.e. surprise, anger, or dismay. These notes were important given that these would not have been obvious when listening back to the audio tapes. These were meant only as reminder points for me to reflect upon after the focus groups and to consider as possible emergent themes before moving forward on to the next group. Some of these notes allowed me to reflect within the focus group itself and ask further questions or clarify points around specific statements, or record when agreement was voiced. While these notes were helpful, I was mindful of the fact that I could not remember everything that was said in each focus group and could not have noted every interesting point, and so while these acted as a guide, listening to the tapes (often in the car on the way to work) and reading the transcribed interviews was essential.

- It has been claimed that a researcher can best get to know their qualitative data by transcribing it themselves (Braun and Clarke, 2006). However, working full time and completing a PhD this was not feasible. I commissioned an outside agency to transcribe my focus groups, but I did re-listen to all focus groups in turn and out of turn to remind myself of the interactions statements made and to start to highlight possible themes within. I made additional notes during this stage and started to organise my thoughts into different emerging themes. I had already decided to organise the data into each focus group question. Listening to the voices in the focus group more than once, while time-consuming, proved invaluable in identifying themes. Listening to actual voices as a useful way of remembering the actual individuals and how they responded and reacted during each focus group.

- Once these notes had been made, I took a break. The transcripts took some weeks to come back, and during this time a break from the data and possible themes was helpful. I was able 
to progress to the transcripts refreshed and began to read every page to identify common themes. I did not read my original notes or audio notes until after I had read the transcripts to ensure I was not biased toward already identified themes. Once I had read the transcripts, I began to highlight different themes using different colours pens and began to summarise significant statements within these themes; which can be seen in appendix 3 . If there were any particularly interesting areas of text I would re-read them while listening to the audio, to ensure that I was able to note the inflexion of the participant and the reaction of those present; this happened regularly. I took regular breaks during this time and kept re-visiting both the audio and the written text and reflecting upon alternatives for the themes I had identified; to ensure I was not biased. I did this separately for each focus group and then compared my notes for each focus group question with each focus group. In addition to this, I organised printed transcripts into focus group questions for all focus groups so that I could read and consider possible themes concurrently.

- After this point, I was satisfied that common themes had emerged. I further reviewed any separate interesting points made by individuals to see if they were considered under other themes and began to analyse and explore each theme separately. This whole process was time-consuming, not least because I was working full time. This intermittent approach to coding was useful and allowed me time away from the data itself to think in more depth and re-visit the audio and written data.

This reflective data has been taken from my research diary and the notes. This not only serves to provide the reader with a view of how I analysed, organised, and better understood my data, but also to display the trustworthiness of the qualitative analysis. Noble and Smith (2015) assert that evaluating the quality of research is essential if findings are to be utilised in practice and incorporated into care delivery and being able to evidence trustworthiness is a crucial aspect of this process. To be accepted as trustworthy, qualitative researchers must demonstrate that data analysis has been conducted in a precise, consistent, and exhaustive manner through recording, systematising, and disclosing the methods of analysis for the reader to determine whether the process is Credible (Nowell et al. 2017). This analysis followed the Braun and Clarkes framework, provided examples within the appendix but also gives a step by step description of the processes followed. 
Authors such as Shenton (2002) describe how the reader should know and understand the background of the researcher, their qualifications and experiences that may influence the research. This allows them to understand the possible risk of researcher bias; something which they continue to assert should be reflected upon. I have outlined my background within this thesis and also included a reflective commentary related to researcher bias; specifically inside researcher bias. Noble and Smith (2015) include within their trustworthiness criteria points such as dependability and traceability, and as I have shown within this section, there is a logical, clearly documented, the literature supported process for my analytical process (which is not without its limitations). I have reflected upon this process and have discussed later within this thesis how this PhD process has helped me grow as a researcher. The importance of trustworthiness cannot be underestimated and allows the reader to assess the reliability of the process used to reach the conclusions and provides a rationale for the application of certain methods; which is key in evidence-based clinical practice.

\section{Insider Research}

The concept of 'Insider Research' cannot be ignored within this thesis given that I am a paramedic myself and am also investigating the roles of UK paramedics in the ambulance service in which I still practice. Insider research has been defined as research conducted by people who are already members of the organisation or community they are seeking to investigate because of education, employment, social networks, or political engagements (Coghlan and Brannick, 2005). Conducting research within communities of which one is already a member can be an advantage, but at the same time introduces a range of professional, ethical, and methodological problems for the researcher (Humphrey, 2012).

To discuss the concept of insider research one must compare it with the concept of outsider research; in doing so, two schools of thoughts become evident. Simmel, (1950) argues that research can only truly be objective if the researcher is an outsider and can be unbiased by internal politics and the need to retain existing relationships. This view is opposed however 
by Merton, (1972) who argues that if an outsider takes up a research which is not related to their topic area, culture, group and status, they will not be able to understand the issue they are working on and therefore, will find it difficult to justify the study findings. While the debate surrounding insider research continues (Saidin and Yaacob 2016), it is worth noting both the advantages and limitations of this method and considering the implications for this research.

It is argued for example that an advantage of insider research is that the researcher always has a passion about the topic they are working on, which means that they commit themselves to the research despite all the possible obstacles (Saidin and Yaacob 2016). Other advantages include the ease of understanding an insider will have towards a particular topic or social group; which allows them to extract and analyse data better than someone who cannot relate to them (Bonner and Tolhurst 2002). Furthermore, the researcher's familiarity with the cultural and political structure of an organisation will help them to save time in trying to understand identified issues as they already have some knowledge regarding the issue (Smyth \& Holian, 2008). Insider researcher often allows a researcher privileged access to participants and important data (Drake, 2010) and allows the insider's information to explore the sometimes-hidden nature of organisations and professions (Hanson, 2013). While these points are valid examples of how insider research can be advantageous, they are also the foundation for some of the limitations of such research.

Insider research can be heavily biased by the researcher's familiarity with the topic or setting and can produce data which fits a pre-conceived hypothesis and which does not robustly challenge the data gathered. Drake (2010) also suggests that research inside one's workplace is likely to be on a small scale involving few people, and it can cause problems for the researcher upon completion, as they must live with the consequences of their findings and how they are interpreted. This can again lead to bias findings, although this can depend upon the role of the researcher within the community or organisation. Much of the literature speaks of the need for interviewers to be neutral in qualitative work, something which can be altered by insider research. The researcher may attempt to be neutral but 
cannot guarantee the feelings and perceptions of the research participants, and tension can be experienced between multiple roles (Hanson, 2013). This power imbalance may, in turn, lead to what Darra (2008) terms 'watering down'. This occurs when the reporting of aspects of practice are diluted, especially when it may reflect badly upon either the participants or the researcher. Alternatively, the participant may wish to present an ideal impression of themselves or their colleagues, again, affecting the veracity of the research findings.

At the time of this research, I was a manager within the sample ambulance service, albeit in a very specific area of clinical practice and a lecturer practitioner in subjects including but not limited to child safeguarding. This may have influenced how respondents and participants answered questions posed to them. This data, in turn, could be susceptible to unconscious researcher bias as conflicts between my corporate, professional, and academic roles influenced each other and the findings. Humphrey (2012) argues that it is incumbent upon insider researchers, along with their supervisors and staff on ethics committees, to become cognizant of the potential risks to anticipate and avoid them or ameliorate their effects. The aim is to become risk-aware rather than risk-averse. By considering the risks associated with insider research a transparent and reflective approach can be taken to how it is undertaken.

This brief critique of the concepts of insider and outsider research sets out for me, as a novice researcher, the advantages and disadvantages of researching one's own practice. As an exercise, this not only highlighted the perils and pitfalls associated with these approaches but clarified the professional, ethical and methodological issues associated with both. By weighing up these advantages and disadvantages associated it enabled me to feel confident that my decision to undertake this research as an insider was the best approach to address the research questions.

I made efforts to reduce the possible risks associated with insider research and reflected upon this process once completed. To ensure there was not a power imbalance between the participants and me, my Swansea University email was used and not my ambulance 
service email. This served to detach me from my position as a manager in the ambulance service; indeed, some participants did not know I worked for the same ambulance trust. When conducting the interviews, I did not wear my uniform and thus did not wear my epaulettes with my rank displayed. Where possible, I used local research champions to organise and communicate with participants to cut down any previous mention of workrelated roles, but I did introduce myself as a paramedic. Participants from both the quantitative and qualitative samples may have viewed me as an authority on child protection, and this could have induced participants to provide the 'correct' answer rather than the most candid and honest answer, although this was not evident in the qualitative work, where participants spoke very freely.

Although I started the research with an idea in mind of the possible results, because of forming the research questions, I was surprised and interested throughout the process at some of the differences compared with my original ideas. Upon reflection, this served to remind me that I could not pre-empt the research results and that I did not speak for all paramedics. Just because I am a paramedic does not mean I am like all paramedics. My wife is also a paramedic, and this relationship constantly reminded me that I do not think like all paramedics and that different experiences form and influence the way we perceive our roles and practice our profession as individuals. Discussing this research with her throughout the six years introduced new concepts and areas of enquiry, and also served as a reminder not to contaminate the analysis and discussion with my views of how practice should be or is in real life. When making notes in a focus group, I did not discount anything because I did not think it was relevant. I wrote as much as I could equally have explored as much as I could. Having supervisors who themselves were not paramedics helped this process too. Instead of resting upon a concept or theme which another paramedic may have done or may have too readily accepted, they constantly questioned findings and almost acted as an outside researcher element to this research. There is always a possibility that unconscious bias could affect a researcher's work, however, I feel that by constantly being challenged by differing views throughout this research I was able to remain objective. I was 
informed by my practice, training, education, and role as a lecturer, but I do not feel that I allowed this to overwhelm my research.

\section{Context / sample / participants}

While it is true that many grades of staff exist within UK ambulance services, this research has focused solely upon paramedics. Paramedics are registered HCPs, who after undertaking a programme of study are eligible to register with the HCPC. The title of paramedic is 'protected' under Article 39 and 39A of the Health and Social Work Professions Order (2001), with those falsely using it being guilty of a criminal offence. The use of this protected title conveys a minimum national standard, which does not exist to the same extent with other grades of staff, such as emergency medical technicians (EMTs) and emergency care assistants (ECAs). There are significant variances in educational and training standards across the UK for EMTs and ECAs and as such, comparison and generalisation is arguably more difficult.

There has been a change in the way in which paramedics are trained and educated across the UK within the last 20 years, but there has remained a minimum standard since 2001. In addition to paramedics being more comparable across the UK, they have a legal and ethical 'duty of care'. Sibson (2011) states that a 'duty of care' is an obligation on one party to take care to prevent harm being suffered by another, and is based in common law. This requires paramedics to adhere to reasonable standards of care at all times. Similarly, the Children Act 2004 , and specifically section 11 , places duties upon ambulance services and those who work for them to ensure that they consider the need to safeguard and promote the welfare of children when carrying out their functions (Children Act 2004, Section 11.2a). These legal responsibilities fall almost always if not entirely to paramedics, both as legally registered HCPs and also as the individuals clinically in charge in most cases. This research focuses on those with the most responsibility, whether legally, ethically, or clinically. While lower grade clinical staff whom paramedics supervise do receive some child protection training, they are not legally registered and arguably are not as legally accountable under specific legislation due to the presence of a senior legally registered clinician. 
All participants for both phases of data collection were invited from a UK ambulance service covering $20 \%$ of the land mass of England; including both large rural and urban area. The service in question employs over 4,000 mainly clinical and operational staff (including paramedics) and serves a total population of over 5.3 million. In addition to this population.

As the researcher works for the ambulance service, a convenience sampling approach to recruitment was used. This allowed the researcher ease of access both digitally and physically to various locations and staff groups. Ethical approval was gained from Swansea University and the ambulance service; however, being employed by the ambulance service meant that it was easier for the researcher to navigate internal governance processes. For example, the use of internal email, intranet pages, internal Facebook pages, and weekly bulletins were used to recruit participants. While there are limitations to convenience sampling methods, the sample ambulance service itself is not dissimilar to all others in the UK. The number of square miles covered means that the population served, demography, topography, and geography, are similar to many other different UK ambulance services. Arguably, this similarity validates the generalizability of any results, despite being identified in just one ambulance service.

\section{Questionnaire respondents}

Out of a possible sample size of 1,679 paramedics within the sample ambulance service at the time of data collection, 469 responses were received. Of these 469 responses, 193 were removed due to partial completion, which left a final sample size of 276 (16.4\%).

Table 1: Gender

Frequency, and percentage of gender of respondents

\begin{tabular}{ll}
\hline Gender & Frequency (\%) \\
\hline Male & $152(55.1)$ \\
Female & $124(44.9)$ \\
Total & $276(100)$
\end{tabular}

Table 1 shows the frequency distributions of males and females were very similar, with 26 more males than females. 
Table 2 - Age

Frequency of age groups of respondents

\begin{tabular}{ll}
\hline Age Group & Frequency (\%) \\
\hline $18-20$ & $1(0.14)$ \\
$21-29$ & $48(17.4)$ \\
$30-39$ & $86(31.2)$ \\
$40-49$ & $100(36.2)$ \\
$50-59$ & $39(14.1)$ \\
60 or older & $2(0.7)$ \\
Total & $276(100)$ \\
\hline
\end{tabular}

Table 3 - Educational and Career Profile

Frequency of highest level of paramedical related educational qualification

\begin{tabular}{ll}
\hline Level of education & Frequency (\%) \\
\hline IHCD Certificate & $113(40.9)$ \\
$\begin{array}{l}\text { Diploma Higher Education } \\
\text { or Foundation Degree }\end{array}$ & $116(42.0)$ \\
Batchelor of Science & $37(13.4)$ \\
Post Graduate Certificate & $6(2.2)$ \\
Master's Degree & $4(1.4)$ \\
Doctorate or PhD & $0(0.0)$ \\
Total & $276(100)$ \\
\hline
\end{tabular}

Table 3 shows that $59.1 \%$ of respondents had undertaken higher education qualifications, on which CP may have been taught in more depth than in previous IHCD courses. 
Table 4 - Years of Qualification

How long have you been qualified as a paramedic?

\begin{tabular}{ll}
\hline Years of qualification & Frequency (\%) \\
\hline $0-14$ & $224(81.1)$ \\
$\geq 14$ & $52(18.8)$ \\
Total & $276(100)$
\end{tabular}

Table 4 indicates the how long respondents had been qualified as paramedics.

\section{Table 5 - Years of service}

What is your length of service in all ambulance services and all roles (not just paramedic)?

\begin{tabular}{ll}
\hline Years of service & Frequency (\%) \\
\hline $0-14$ & $170(61.6)$ \\
$\geq 14$ & $106(38.4)$ \\
Total & $276(100)$ \\
\hline
\end{tabular}

Table 5 indicates the length of combined service of respondents in all services and all roles (not solely as a paramedic). It is common for paramedics to have worked at lower grades in ambulance services, through which experience can be gained, before qualification. It is reported in this manner due to an error in the questionnaire design and thus in data collection, which may have led to the over reporting of years. 
Table 6 - Participant Roles

Respondents' current roles in the ambulance service

\begin{tabular}{ll}
\hline Current role & Frequency (\%) \\
\hline $\begin{array}{l}\text { Paramedic / Clinical Advisor/ Supervisor / } \\
\text { Trainer / Officer / or any other role }\end{array}$ & $241(87.3)$ \\
$\begin{array}{l}\text { Paramedic Practitioner / Emergency Care } \\
\text { Practitioner/ Advanced Paramedic }\end{array}$ & $27(9.8)$ \\
Critical Care Paramedic & $2(0.7)$ \\
Dual Qualified Nurse and Paramedic & $6(2.2)$ \\
Total & $276(100)$ \\
\hline
\end{tabular}

Table 6 demonstrates that most of the paramedic respondents $(87.3 \%)$ were not currently acting in a senior clinical role. Senior or more diverse roles, such as critical care paramedic or advanced paramedic, tend to deal less with children and young people and more with adult populations. 


\section{Focus Group Participants}

In all, 7 focus groups were conducted with a total of 25 paramedics (as seen in Table 7).

Participants were from different areas of the ambulance service, representing differences in the communities they served regarding geography, demography, and topography (inner city and rural).

\section{Table 7}

Demographics of focus group participants

\begin{tabular}{|c|c|c|c|c|}
\hline $\begin{array}{l}\text { Focus } \\
\text { Group }\end{array}$ & Gender & Age (years) & Years Qualified & Length in minutes \\
\hline \multirow[t]{4}{*}{1} & Male & 44 & 2 months & 47.08 \\
\hline & Female & 38 & 8 Years & \\
\hline & Female & 28 & 7 Years & \\
\hline & Female & 25 & 6 Years & \\
\hline \multirow[t]{3}{*}{2} & Female & 43 & 17 & 55.41 \\
\hline & Female & 33 & 18 months & \\
\hline & Female & 30 & 3 years & \\
\hline \multirow[t]{4}{*}{3} & Female & 59 & 17 & 47.48 \\
\hline & Female & 43 & 5 & \\
\hline & Female & 42 & 11 & \\
\hline & Female & 14 & 6 & \\
\hline \multirow[t]{3}{*}{4} & Female & 45 & 5 & 50.53 \\
\hline & Female & 42 & 6 & \\
\hline & Female & 40 & 16 & \\
\hline \multirow[t]{4}{*}{5} & Male & 53 & 17 & 58.54 \\
\hline & Male & 45 & 10 & \\
\hline & Female & 43 & 12 & \\
\hline & Female & 34 & 3 & \\
\hline \multirow[t]{4}{*}{6} & Male & 50 & 17 & 43.08 \\
\hline & Female & 50 & 17 & \\
\hline & Male & 38 & 10 & \\
\hline & Female & 31 & 6 & \\
\hline \multirow[t]{3}{*}{7} & Male & 45 & 13 & 59.09 \\
\hline & Male & 42 & 7 & \\
\hline & Female & 38 & 11 & \\
\hline Total & 25 & Mean 40 & Mean 9.2 & 361.21 Mins \\
\hline
\end{tabular}

Table 7 shows the frequency and demographic details of focus group participants. 
There is little consensus on the number of focus groups that need to be undertaken to achieve research validity, and on the size of those groups. Commonly cited guidelines include data saturation and demographic characteristics as key determinants for robust focus group research; however, these suggestions are not supported by empirical data (Guest, et al., 2016). Some authors suggest that there is no recognised gold standard for the number of focus groups one must conduct to provide valid findings (Barbour, 2007, p. 61), and that even one focus group can provide enough findings with enough validity to answer a researcher's questions.

Although data saturation is considered by some as a sign of robust data collection (Braun and Clarke, 2013), it is difficult to estimate prior to undertaking focus groups because the researcher will not recognise that saturation has been reached until the focus groups have been carried out. Topic complexity might also influence the length of time it takes to reach data saturation; the more complex the subject being discussed, the more focus groups are needed (Guest et al., 2015), and the more varied are the views that might be expressed, discussed, and challenged. Child protection is a complex and emotive subject. The demographic diversity of the paramedic population in terms of age, location, educational background, and even role speciality meant that any sample group was likely to be heterogeneous in nature. Similarly to complexity, sample heterogeneity can influence the length of time it takes to reach data saturation (Bartlett et al., 2015), as differing views are expressed, discussed, and challenged depending on the demographic characteristics of the participants.

It was decided to attempt to conduct as many focus groups as possible that comprised as great a variety of groups as possible to attempt to achieve data saturation. These focus groups were based around geography given the size of the ambulance trust and the need for meeting rooms. Geographical variation was also considered an advantage to gain different demographic characteristics. Despite widespread advertising, no more than seven groups of participants came forward. At the time of the research, the sample ambulance service were keen for those undertaking external research projects to complete them before they started their most recently commissioned research so as not to distract or 
exhaust their possible participants. This meant that the researcher had to attempt to gain as many focus groups as possible across a diverse enough practice area within a timeframe that did not overlap with his employer's pre-planned research. It was decided to carry out the initial seven focus groups and assess if data saturation had been achieved after focus group seven had been conducted. If it appeared not to have been achieved, permission would have been requested from the sample ambulance service to continue to attempt recruitment. New themes ceased to emerge towards the end of the fifth focus group; thus, data saturation had been achieved. It was decided to continue to the seventh group, as there was a possibility of more male paramedics participating, which would have been advantageous for the heterogeneity of the sample groups.

\section{Measures and Instruments}

This section outlines the measure the instruments used to collect the data needed to answer the research questions and achieve the overall research aims. In line with the chosen explanatory sequential mixed methods, it outlines the instruments used in order of their use.

\section{Questionnaire}

The questionnaire for this study (the complete version of which can be found in Appendix 4) was based on findings from the in-depth critical review of the literature within Chapter 2, and further developed in relation to Carper's theoretical framework. Measuring the level of knowledge and attitudes of paramedics towards protecting children in this way aims to inform future practice, education, and policy. It comprises 39 separate questions clustered into subject areas. These areas were 1) Safeguarding in practice (training updates, feedback provided), 2) Patterns of knowing (how often types of knowledge were used), 3) Attitudes towards child protection (How relevant various subjects were to practice), 4) Level of confidence (confidence in recognising various issues, overcoming barriers), and 5) participant demographics. The categories used within the questionnaire were informed both by the researcher's knowledge of practice and by the literature itself. For example, the reasons offered for why child protection referrals may not have been completed in practice 
were informed by the researcher's anecdotal understanding of the challenges paramedics face day-to-day in clinical practice, such as there not being enough time, as well as the literature indicating why some referrals are not completed, such as a lack of proof.

The questionnaire was self-administered. This methodological design allows the participant to complete the questionnaire themselves with minimal instruction from the researcher. This method is quicker to administer, relatively cheap, useful in widely geographically dispersed samples, convenient for participants, and relatively free from research bias (Bryman, 2008). The researcher considered it important that participants be given examples of some elements of enquiry, for example, psychosocial determinants of health. Many participants may not have fully understood this term and therefore, terms such as 'effects poor housing have on child development' were used instead to ensure better understanding. The questionnaire was based around the Likert scale, which will be discussed in more detail in the next section.

\section{Attitudinal scale-based questionnaire}

Attitudinal scales are used extensively in public health and social science research (Parahoo, 2006), and many variations exist. Typically, they involve a statement about the attitude being measured and a response arrangement. Using a numeric range, the respondent is asked to indicate the extent to which they agree or disagree with a prescribed statement (Thomas, 2011). Other variations include the use of prescribed answers which have been given a score on a similar numeric range - such as strongly agree, agree, and disagree. This method was used to yield continuous data. It is however, limited somewhat in that it is assumed that the participants' attitudes can be represented and analysed numerically and that all statements and their meaning will be viewed and comprehended ubiquitously.

The chosen instrument for this research was the Likert Scale. Rensis Likert developed this scale in 1931, and it usually comprises five response categories ranging from $5=$ strongly agree to $1=$ strongly disagree. There is much debate among researchers surrounding the optimum number of choices in a Likert-type scale, with some suggesting a preference for seven categories or with an even number of responses (Croasmun and Ostrom, 2011). The 
Likert style scale aims to measure the intensity of feelings about the area in question and is one of the most commonly used techniques for conducting investigations into the attitudes of people and groups (Bryman, 2008). The Likert scale is seen by many as being a useful and reliable tool in social science attitude research projects (Croasmun and Ostrom, 2011). One of the greatest advantages of many attitudinal scales is the simplicity and practicality of their development. Warples (2010) suggests that although simplicity is not always desirable for its own sake, in the case of attitude assessment, it translates into pragmatic values. Warples goes on to assert that Likert-style scales have consistently higher estimates of reliability and greater intuitive appeal among organised decision makers, thus, reinforcing their place within industrial and organisational psychology. The reliability of this instrument can itself be tested to a certain extent, by phrasing statements both positively and negatively (Thomas, 2011), thus, increasing the instrument's robustness. A further advantage of this scale instrument is the ease of its use by respondents. As a wellrecognised instrument, respondents may have used it before in other research, and if not, then its simplicity allows a very brief explanation of its use to be sufficient. For a selfcompleted questionnaire delivered remotely by respondents who may be interrupted by an emergency call at any point, this was seen as an advantage in both the time and speed of the response. As per the sequential research design, the quantitative data would directly inform the design of the qualitative element of this research. It was decided that this would be done through semi-structured focus groups.

\section{Piloting the questionnaire}

Given the complexity of constructing a questionnaire and as the subject matter of this questionnaire has yet to be researched in such depth, it was deemed appropriate to conduct a pilot study. Quantitative studies typically involve a small-scale run of the larger project, which allows the researcher to determine the adequacy of the instructions given for answering the questions and to understand the reasoning for any questions that were missed out. Piloting in effect ensures that the instrument of measurement itself is fit for purpose and operates well (Bryman, 2008). Pilot studies are seen not only as good practice, but are often crucial in relation to research based on self-completion questionnaires due to 
the amount of variability involved (Holloway and Wheeler, 2009). The research and development department facilitator of the sample ambulance service was asked to identify paramedics from her existing database, whom she thought might be willing to pilot the questionnaire. Return of the pilot questionnaire itself was anonymous via the online platform as originally planned, and the questions below were returned via the facilitator to ensure anonymity, and thus increase the potential honesty of suggested changes.

\section{Pilot Study One}

The questionnaire, along with the questions below, was sent to five paramedics throughout the ambulance service as part of phase one of the pilot study. The results were used to inform a redrafting of the questionnaire.

1) Were you able to understand all the questions easily; if not, please state which ones, and why?

2) How long roughly did it take you to complete this survey?

3) What are your thoughts about the layout and design of this survey?

4) Are instructions for completing the survey clearly written?

5) In terms of anonymity, how comfortable were you completing this survey?

6) Is there anything about this survey that you would change?

7) Are there any questions you would not complete and if so why?

Although the data collected from the questionnaire was in of itself interesting, the pilot study was primarily to test the efficacy of the questions themselves. Feedback was generally positive, with comments such as those below informing minor changes.

\section{[Pilot Participant 4]}

"The only one that felt a little uncomfortable was the one that asked for reasons why referrals hadn't been made when perhaps they should have (24) - but it is non-specific. On a technical point I think this question should have accepted more than one answer, but it didn't for me." 
And

"I would alter one of the latter questions which pertains to the pathway to Paramedic qualification to include Ambulance Care Assistants as a starting point in one's career. ECAs are a more recent advancement."

\section{[Pilot Participant 1]}

"I would also allow more than one choice for reasons behind not making a child safeguarding referral. Perhaps you have, but I was only able to give one reason."

Phase one respondents also stated that completing this questionnaire took around 10-15 minutes; the author deemed this to be too long given the workload and time constraints on paramedics currently working in practice. Some questions were later removed without undermining the integrity and overall aims of the research.

\section{Pilot Study Two}

After a redraft of the questionnaire, a different group of five paramedics were asked to undertake a pilot to ensure its efficacy. Ten pilot respondents in total were deemed sufficient to ensure validity in the piloting process and findings, while also ensuring a small enough pilot sample so as not to limit the potential end sample size or those whose responses may be swayed by prior completion of draft questionnaires. Feedback from the second pilot was again generally positive, with comments similar to those of pilot study 2 , resulting in some minor changes to the layout and question structure only. After these changes, the questionnaire was again sent to the chair of the research ethics committee and confirmed as being compliant with the ethical approval due to the removal of certain questions and the relatively few changes to the existing questions.

\section{Focus groups}

Focus group research is a method of interviewing that usually involves at least four participants and aims to collect data through group interaction about a topic determined by the researcher (Bryman, 2008). Focus groups give research participants the opportunity to describe what is important to them through self-discovery and exploration, coupled with the ability of the researcher to explore participants' reasons for acting in a certain way 
(Bryman, 2008; Holloway and Wheeler, 2009; Doody and Noonan, 2013). Focus groups were chosen as a qualitative method because of their ability to provide a means by which data can be organised and interpreted without losing the richness and individuality of responses (Stuckey 2013); paramedics experience many different things in their clinical practice that cannot be adequately brought to light through questionnaires alone. Mack et al. (2005) argue that qualitative research produces findings that are applicable beyond the immediate boundaries of the study, which is important given the evolving nature of paramedicine within the community and the many other professions who themselves may not fully understand their role within child protection.

\section{Focus group structure}

Focus groups can be structured or semi-structured. By using questions that are semistructured, the researcher has more flexibility to gain an in-depth insight into often personal or complex anthropological aspects of life, such as, in this instance, the culture within ambulance services and the paramedic profession. Semi-structured approaches involve the use of predetermined questions where the researcher is free to seek clarification and explore issues that arise spontaneously, which often have not initially been considered (Doody and Noonan, 2013). The ability to deviate from scripted questions when necessary can also lead to the in-depth analysis of responses of great validity, which are often emotional and based deeply in experience and individual reality (Baumbusch and Clark, 2010).

The quantitative statistics directly informed the qualitative element of this research as per the sequential research method. The descriptive data informed the design of the semistructured research question, as seen below.

1. How do paramedics perceive their role in child protection?

2. How do paramedics perceive the training they receive on child protection?

3. How confident are paramedics in child protection? 
4. What barriers exist to paramedics undertaking their role in child protection?

5. Can any ways of improving paramedic's practice in child protection be identified?

By answering these questions, which were based on data from the quantitative element of this study, the overall objective to inform future paramedic practice can be achieved. The results chapter will outline the statistics involved. However, it was thought helpful to show the sequential underpinning of the procedure that took place.

The focus group questions were:

\section{1) What do you think a paramedic's role in child protection is?}

This question was asked because of the mixed responses to the relevance of certain areas of practice, which made it somewhat unclear as to what paramedics thought their responsibilities were. In exploring this further, it was hoped that the research question 'How do paramedics perceive their role in child protection' could be answered in sufficient detail to achieve the overall objective.

\section{2) Do you think paramedics have enough child protection training?}

This question was asked because most of the respondents agreed that there was adequate training in child protection in place, yet in contrast, very few respondents said that they were very confident in recognising signs of sexual abuse, CSE, and FGM. This raised the question as to whether the training was indeed adequate. Considering that the research questions mentioned both training and barriers to undertaking child protection, the exploration of this element could contribute to the overall objective being achieved.

3) Why do you think paramedics may lack confidence in recognising signs of sexual abuse, child sexual exploitation, and female genital mutilation?

This question was asked because very few respondents said that they were very confident in recognising signs of sexual abuse, CSE, and FGM. This contrasted heavily with other areas of practice, for example, the ability to recognise physical abuse. This contrast in response was 
unexpected and needed to be explored further through focus group discussion. There may have been a barrier to confidence in this specific sexual element of child protection, which thus warranted further exploration.

\section{4) What would make you decide to complete a child protection referral?}

This question was asked because a hierarchy in the 'way of knowing' paramedics employed when making decisions about child protection referrals was identified. Larger proportions of respondents, for example, used aesthetic (gut feeling) knowledge in comparison with those using factual knowledge. The overall objective of this study is to inform future paramedic practice, education, and policy, and if it is made clear why paramedics use different types of knowing, then future educational packages may be able to use this in their design. The exploration of this question through focus group discussion contributes to explaining this hierarchy of knowing, and answers a research question regarding ways of improving future practice, thus, contributing to the overall aim of this study.

\section{5) What are the reasons why paramedics may not submit child protection referrals?} Many themes were highlighted indicating why paramedics do not submit child protection referrals. While these reasons give an indication why referrals are not made (not enough time, for example), they are not explanations. A more personal and elaborative approach, facilitated by focus group discussion, was needed to explain these reasons and why they inhibited referrals being made. The frequency of many of these themes was unexpected, and so the focus groups were used to explore these further. By doing so, this addressed the research questions which referred to barriers in the implementation of child protection as well as ways of improving practice, thus contributing to the overall aim of the study.

These focus group questions address the need to explore unexpected results within the quantitative data, and address the need to ratify the quantitative data, through a deeper more personal group-facilitated exploration. Such elaboration and explanation is done not solely for the sake of explanation, but also to contribute further to answering the research questions. In doing so, it is envisaged that the overall objective of informing future paramedic practice, policy, and education can be achieved. The results chapter will outline 
the statistics involved. However, it was thought helpful to be able to show the sequential underpinning of the procedure that took place and for which ethical approval had been obtained.

\section{Focus group limitations}

Despite focus groups being well established and increasingly popular in health research (Jayasekara, 2012), they are not without their limitations as a method of data collection. One such limitation is the facilitation of focus groups by novice researchers, who may not know when to prompt or seek clarification of points, due to their inexperience (Madill, 2011). The researcher himself was a novice in conducting semi-structured focus groups and so undertook some workshops to help overcome this disadvantage. This increased his confidence, allowed him to discuss feedback with his peers, and prepared him to conduct his own focus groups.

The group dynamic of focus groups is itself considered a disadvantage by some, depending on the participants present. Some members of focus groups might inhibit others from speaking or may be very forthcoming with their views. This may, in turn, deter other group members from speaking, especially if they hold an alternative viewpoint. In a similar vein, if a focus group consists of members who are not all equal in terms of rank, superiority, or managerial responsibility, junior staff may be deterred from voicing certain views in front of senior or managerial staff. All the staff within each focus groups were of similar roles and ranks, possibly negating this limitation. Having a group of similarly ranked professionals, however, introduces the risk of providing a platform for a collective 'moaning session', which, while interesting to a certain degree, could become unproductive. If a moderator or facilitator were to intervene in this collective moan, questions might be raised over research bias. This highlights a limitation, but also demonstrates how complex it can be to consider certain methods.

Despite the recognised limitations of focus groups, the researcher chose to use semistructured focus groups because the advantages outweighed the possible limitations. 
Advantages of focus groups include the ability to identify new emerging concepts and expand upon existing ones. This usually takes place in an environment in which participants feel more comfortable and open to sharing their views, and provides an opportunity where opposing views may be presented and discussed. Opposing views may result from different experiences or different meanings of events being presented, depending upon age, length of service, and educational route. Focus groups provide a platform for these differences to be explored and compared. Other advantages include focus groups' usefulness in describing processes and systems and integrating perspectives and viewpoints. A better understanding of the internal processes of an organisational or professional culture can be sought from an understanding of how people interpret events, relationships, and theories (Huston and Hobson, 2008; McParland and Flowers, 2011). From a practical perspective, in this research, the use of focus groups meant less cumulative time was taken up with interviews, and fewer hours of recording had to be transcribed, representing a significant financial saving. Furthermore, the geography of the sample ambulance service $(20 \%$ of the land mass of England) meant that the most data could be gathered from the fewest possible journeys, thus also representing a significant saving of cost and time. Many of the limitations of the use of focus groups are already well-recognised limitations in qualitative research and did not specifically result from this researchers' design or selected sample. The next section explores how these instruments and methods were used to collect the data required.

\section{Procedure}

\section{On-line questionnaire delivery}

The questionnaire was distributed to all staff in an email, which included an introduction, explanation, and an https link that then redirected the user to the platform www.surveymonkey.com. Hoonakker and Carayon (2009) state that due to rapid advances in computer technology, the use of the internet for questionnaires or the distribution thereof has increased dramatically, with millions being sent out each year and researchers benefiting from access to millions of potential respondents. There are advantages to using 
online-questionnaire-based methods in comparison with the more traditional postal method. However, there is little noticeable difference in response rate between the two methods when compared with specifically targeted respondents (Ritter et al., 2004; Hoonakker and Carayon, 2009). Despite this, however, the researcher viewed the online delivery as an advantage given the geographical size of the ambulance service, the alternating and opposing shift patterns of many paramedics, and the lack of researcher influence. Similar benefits were recognised in the use of the questionnaire platform itself (www.surveymonkey.com). Despite its rather humorous name, the platform is a wellrecognised, serious, and excellent evaluation tool. It is considered easy to configure, has a rich array of options, and can offer secure storage and easily downloadable options of raw data to external statistical analysis packages, such as the Statistical Package for the Social Sciences (SPSS) (Gorden, 2002). Further advantages of online distribution include its ease of accessibility, with all the ambulance service staff having access to the internal email system. Online distribution is more economical in terms of time and money, and data can be collected, collated, and analysed very quickly (Bryman 2008, Hoonakker and Carayon, 2009).

Eysenbach and Wyatt (2002) remind us that there are still limitations to online distribution and methods, which are discussed within the limitations chapter of this thesis. Despite these limitations, which were far outweighed by the multiple advantages, this method is still considered an important healthcare research tool for the study of attitudes, beliefs, behaviours, and practice patterns of healthcare staff (Leece et al., 2004). It was seen as a suitable method to collect data with the aim of informing future paramedic practice, education, and policy.

\section{Distribution of the questionnaire}

As mentioned previously, the questionnaire was distributed using the online platform www.surverymonkey.com. It was hoped that by using advertisements in the form of internal weekly bulletins, internal Facebook pages, emails, and internal intranet pages (Appendix 5), participation would be maximised. Staff within the Research and Audit Department of the sample ambulance service were also employed to send out reminder emails to all 
paramedic staff. These methods meant that the researcher himself did not ask any respondents directly. This was deemed an advantage given the nature of some of the questions within the questionnaire and the need for anonymity. To ensure respondents did not complete the questionnaire more than once, the option to choose only one IP address per participant was selected, making sure that single respondents could not complete the questionnaire twice. Respondents could, of course, have logged on from home (on their own IP address) and thus completed it both in an ambulance service building and at home; however, this limitation is general to most online questionnaires, and the researcher thinks that it is unlikely to have occurred.

The questionnaire went live on 4 March 2015 and remained open until 12 April 2015.

\section{Conducting focus groups}

An advert was placed at the end of the questionnaire asking for expressions of interest from participants who would possibly willing to take part in focus groups. Similarly to the quantitative arm of this research, adverts were circulated on internal email systems, internal Facebook pages, and weekly bulletins. In all, 7 focus groups were conducted, with a total of 25 participants. The researcher travelled to seven separate sites (which will remain anonymous) and undertook recorded semi-structured interviews. The focus groups were recorded using two separate Dictaphones, the second being used just in case there was a technological failure with the first. An external transcription service then transcribed the recordings and returned the transcriptions securely password protected to the researcher.

A register was kept, and operations officers were told of the focus groups taking place to ensure that health and safety procedures were adhered to. Each location had a member of staff (registered HCP) on site to assist if a participant became upset or distressed (which nobody did), and access to incident reporting methods were available had this been the case. These elements were all considered part of an ethical data collection method, which is explored in the next section. 


\section{Research ethics}

Research ethics is a key tenet of any research, but especially research involving vulnerable groups, such as those found in healthcare settings. Schnell and Heinritz (2006) state that research ethics addresses the question of which ethically relevant issues caused by a researcher's intervention or procedure can be expected to affect the people with or about whom they research. Research ethics involves the protection of those who participate in that research and whom that research may affect. Beauchamp and Childress (2008) devised four ethical principles for research:

- Autonomy: Participants' values and decisions should be respected.

- Non-maleficence: Researchers should avoid harming participants.

- Beneficence: Research should produce some positive and identifiable benefit and not be conducted solely for its own sake.

- Justice: All participants should be treated equally.

These principles were originally being devised for biomedical research but have been adopted within social science research, and now form the foundation of multiple research and governance-guidance frameworks. Griffiths (2011) explains that research governance provides regulations, principles, and standards of good practice to ensure that research studies are suitably planned and that they demonstrate ethical appropriateness. The frameworks to which this research adhered were congruent with the sample ambulance service Research Governance Policy, the Research Governance Framework for Health and Social Care in Wales Welsh Assembly Government (WAG) (2009), and the National Institute for Health Research, Guide to Good Clinical Practice (2016). These frameworks set out the responsibilities of participants, researchers, investigators, universities, organisations, and healthcare professionals. As the researcher is a registered HCP, this research was also conducted in line with the researcher's responsibilities under both the HCPC standards of conduct, performance, and ethics (2012), and the Standards of Proficiency (2012). Latcham (2013) explains that medical ethics and moral philosophy attempt to unravel the rights and wrongs of different areas of healthcare practice and use philosophical analysis to ask 
questions about values, rights, and wrongs, and about what ought or ought not to be done in each situation. Basic considerations such as whether this research is valid, were rigorously planned, and were conducted before, to guard against wasting participants' time and effort.

This following element of the chapter refers both to the quantitative and qualitative research conducted. Separate research applications were made to the sample ambulance service and to Swansea University College of Human and Health Science and College of Medicine Research Ethics Committee. Both sets of applications were granted.

\section{Participant wellbeing}

The dignity, rights, safety, and well-being of participants must be the primary consideration in any research study (WAG 2009 p. 8), especially in studies investigating potentially upsetting and emotive factors, such as child abuse. Congruent with both of Beauchamp and Childress' (2008) principles of autonomy and non-maleficence, Bryman (2008) posits that most people think that research that is likely to harm participants is unacceptable. Potential harm first needs to be defined and then mitigated and controlled. The British Sociological Association (BSA) (2017) states that researchers should anticipate and guard against any consequences for research participants, which can be predicted to be harmful and need to consider carefully the possibility that the research experience may be a disturbing one. Both of the methods used in this research were reviewed for any possible risks, and as the application for ethical approval shows, supportive mechanisms were put in place to protect against the possible risk of harm. Participants were made aware of these during the consent process. The normal ambulance service counselling services were advertised as well as outside agency services for emergency personnel should any recounting of events cause discomfort.

This research meant that participants were likely to draw upon situations relating to exposure to child abuse, neglect, murder, and death. Farrimond (2013) describes such topics as 'sensitive subjects' and considers them to be ones which are not part of everyday discourse - along-with criminal activities, and personal topics such as death, and illness, 
which participants may empathise with. This research had the potential to be upsetting, uncomfortable, and stressful, and could have led participants to query their previous decision making. This had the potential to result in emotional, psychological, economic, and even perhaps, although highly unlikely, reputational, legal, and physical harm. Those who took part in the focus group research were all signposted on multiple occasions to generic counselling services, coupled with the sample ambulance services own in-house counselling services. Safeguards fell within the ambulance service Research Governance Policy framework, which stipulates the importance of good risk management, and the maintenance of risk identification, reduction, and elimination where reasonably practicable through appropriate control mechanisms. In addition to this, all focus groups were conducted on ambulance service premises, and thus adhered to their own health and safety at work guidance, ensuring participant well-being on multiple levels. The researcher himself was aware that he could be affected by the exposure to multiple sources of upsetting and disturbing information, and therefore, counselling services were also available to him through the above sources, in addition to those of Swansea University College of Human and Health Sciences. Furthermore, he and his supervisors reflected upon focus group exercises to discuss any of the possible harmful effects. The supervisors were all aware of the focus group timetable and location, and the number of participants, to ensure the researcher's safety and wellbeing.

\section{Informed consent}

Informed consent means that participants have voluntarily permitted to take part in the research while being fully aware of what is involved and of the potential risks and benefits inherent to the inquiry (Holloway, 2008). The Nuremberg code (1946 P1) states, "The duty and responsibility for ascertaining the quality of the consent rests upon each individual who initiates, directs or engages in the experiment. It is a personal duty and responsibility which may not be delegated to another with impunity".

Before respondents completed the online questionnaire, they were presented with an online information page, which stated that consent would be assumed if the participant continued to participate. Herring (2012) states that for there to be an effective consent to 
treatment, it is necessary to show that the person is competent, sufficiently informed, and not subject to coercion or undue influence. These principles arguably apply to many types of research as well as clinical treatment. Those who agreed to participate in this research were all registered HCPs and were presumed to have full mental capacity, as deemed by the Mental Capacity Act 2005, and all had the cognitive ability to be professionally registered.

Before participants undertook the focus groups, they were presented with an information sheet (Appendix 6) and consent form (Appendix 7). No participant could proceed with the focus group research without signing to agree to consent and show understanding. This was done in the absence of any coercion or undue influence, and was reinforced by the provision of details of someone independent whom participants could approach if they felt the need to complain.

\section{Data protection and confidentiality}

Data protection and confidentiality from a key part of modern health and social care, and have been enshrined in both political and legislative literature; such as the Data Protection Act (1998), the Declaration of Helsinki (2008), and the ambulance service Research Governance Policy. While this research did not include or require data from patients themselves, or from patient records, Bryman (2008) states that researchers and organisations, nevertheless, must protect the integrity and confidentiality of subjects' data (subjects in this instance being paramedic participants). Such data include personal information, names, contact details, and expressed opinions, and a clear policy relating to who has access to such information is needed. Griffiths (2012) states that the Data Protection Act 1998 specifically ensures that data cannot legally be collected, stored, retrieved, or organised without a person's consent. Importantly also for researchers, data for which consent has been obtained for one research reason cannot then be used for another without expressed and informed consent. The ambulance service recognised that records and information must be managed, handled, and protected in accordance with the requirements of the Data Protection Act 1998 and other legislation. This is not only to serve its business needs, but also to support the provision of the highest quality patient care and to ensure that individuals' rights in respect of their personal data are observed. Given that 
this research was being conducted within the ambulance service the application for research ethics needed to show how this was going to be achieved. The raw data collected via the questionnaires and from the recordings and transcripts from the focus groups were kept on password-protected computers in a password-protected file, with access restricted to the researcher himself and his supervisory team. Any information sent by email was done so using encryption software, which included communication from the transcription service. This author ensured that there was mention of data protection within the focus group participant information sheet, and that the transcribers of the focus group data confirmed that they would conform to the Data Protection Act also, as well as ensuring that transcripts themselves did not include participants' names. Audio and written data were kept in a locked cabinet, and / or password-protected and encrypted computer files, with identifier codes, which were kept similarly but separately.

\section{Reciprocity}

This researcher was constantly aware that by participating in his research, be it through the completion of the questionnaires or by participation in focus groups, participants were ensuring that he could work towards the completion of his PhD. It would have been financially prohibitive to compensate those who completed questionnaires for their time. However, he did provide each participant of the focus group with a $£ 10$ gift voucher for a group of high street stores (funded by Swansea University) in recompense for a more substantial use of their time and effort. Light refreshments were also available so that participants would feel more comfortable.

Ethical approval was granted by the ambulance service and ethical approval was also obtained from the College of Human and Health Science and the College of Medicine Research Ethics Committee, Swansea University on 27 October 2014. Further approval was given from the chair of the committee after the re-wording or removal of some questions following the findings from a pilot study. 


\section{Chapter Four: Results and Findings}

This chapter outlines both the quantitative results and the qualitative findings. The research aims informed the design of the in-depth review of the literature, which informed the design of the questionnaire. The design of the questionnaire in turn informed the design of the focus group questions. This sequential design continues in this results and findings chapter and shows an integrated approach to the presentation of data. Driscoll et al. (2007) highlight that there is a lack of recognised consensus on the presentation of mixed methods data, with different options having different strengths and weaknesses. Rees and Rees (2017) comment on how researchers often have to wrestle between clustering all their quantitative and qualitative data into clearly defined separate chapters and risk having a fragmented approach, versus having an intergraded approach to data presentation which can make it difficult for the reader to follow. The overall research design is sequential, and thus integrated in nature, hence the choice of layout. Only statistically significant $(<0.05)$ data are presented within the results; however, all raw data is available.

With this layout in mind, it was thought helpful to remind the reader of the research questions, all of which aim to achieve the overall objective of informing future paramedic practice, policy, and education.

\section{Research questions}

1. How do paramedics perceive their role in child protection?

2 How do paramedics perceive the training they receive?

3 How confident are paramedics are in child protection?

4 What barriers exist to paramedics undertaking their role in child protection?

5 Can any ways of improving paramedic's practice in child protection be identified? 


\section{Question 1: How do paramedics perceive their role in child protection?}

This section outlines the perception of relevance respondents placed upon certain areas of child protection, and how respondents perceived their role in child protection.

\section{Perception of relevance}

The following questions asked respondents to rate the level of relevance of certain subject areas to their role as a paramedic. Independent Sample T-Tests were conducted, and the significant findings are presented.

\section{Table 8}

Relevance of knowledge of physical abuse

\begin{tabular}{ll}
\hline Perception & Frequency (\%) \\
\hline Highly relevant & $198(71.7 \%)$ \\
Relevant & $78(28.3)$ \\
Neither relevant or not relevant & $0(0.0 \%)$ \\
Not relevant & $0(0.0 \%)$ \\
Not at all relevant & $0(0.0 \%)$ \\
Total & $276(100)$ \\
\hline
\end{tabular}

Table 8 shows the frequency of perceived relevance of physical abuse, which has historically been difficult to define.

\section{Table 9}

Relevance of knowledge of emotional abuse

\begin{tabular}{ll}
\hline Perception & Frequency (\%) \\
\hline Highly relevant & $190(68.8)$ \\
Relevant & $83(30.1)$ \\
Neither relevant or not relevant & $3(1.0)$ \\
Not relevant & $0(0.0 \%)$ \\
Not at all relevant & $0(0.0 \%)$ \\
Total & $276(100)$ \\
\hline
\end{tabular}


Table 10

Relevance of knowledge of sexual abuse

\begin{tabular}{ll}
\hline Perception & Frequency (\%) \\
\hline Highly relevant & $189(68.4)$ \\
Relevant & $83(30.0)$ \\
Neither relevant or not relevant & $4(1.4)$ \\
Not relevant & $0(0.0)$ \\
Not at all relevant & $0(0.0)$ \\
Total & $276(100)$ \\
\hline
\end{tabular}

Table 11

Relevance of knowledge of neglect

\begin{tabular}{ll}
\hline Perception & Frequency (\%) \\
\hline Highly relevant & $197(71.3)$ \\
Relevant & $79(28.6)$ \\
Neither relevant or not relevant & $0(0.0)$ \\
Not relevant & $0(0.0)$ \\
Not at all relevant & $0(0.0)$ \\
Total & $276(100)$ \\
\hline
\end{tabular}

\section{Table 12}

Relevance of the knowledge psychological and sociological factors affecting child and family health

\begin{tabular}{ll}
\hline Perception & Frequency (\%) \\
\hline Highly relevant & $115(41.6)$ \\
Relevant & $138(50)$ \\
Neither relevant or not relevant & $19(6.8)$ \\
Not relevant & $4(1.4)$ \\
Not at all relevant & $0(0.0)$ \\
Total & $276(100)$ \\
\hline
\end{tabular}

Table 12 shows the first example of where respondents identified a subject area as not being relevant to their role as a paramedic in relation to child protection. 


\section{Table 13}

Relevance of the knowledge of health promotion and disease prevention

\begin{tabular}{ll}
\hline Perception & Frequency (\%) \\
\hline Highly relevant & $87(31.5)$ \\
Relevant & $148(53.6)$ \\
Neither relevant or not relevant & $29(10.5)$ \\
Not relevant & $10(3.6)$ \\
Not at all relevant & $2(0.7)$ \\
Total & $276(100)$ \\
\hline
\end{tabular}

Table 13 shows the first example of where respondents identified a subject area as not being at all relevant to their role as a paramedic in relation to $\mathrm{CP}$.

\section{Table 14}

Relevance of the knowledge of lifespan development (developmental landmarks)

\begin{tabular}{ll}
\hline Perception & Frequency (\%) \\
\hline Highly relevant & $95(34.4)$ \\
Relevant & $140(50.7)$ \\
Neither relevant or not relevant & $32(11.5)$ \\
Not relevant & $9(3.2)$ \\
Not at all relevant & $0(0.0)$ \\
Total & $276(100)$ \\
\hline
\end{tabular}




\section{Table 15}

Independent sample t-test comparing the sex of respondents with respondents' perceived relevance of knowledge of types of abuse.

\begin{tabular}{llllllllll}
\hline Table & Group & $\mathbf{N}$ & Question & Df & Mean & Sd & T & sig* & Cohen's d \\
\hline A & Male & 152 & $\begin{array}{l}\text { Relevance of knowledge of physical } \\
\text { abuse to paramedic practice }\end{array}$ & 274 & 1.34 & .474 & 2.172 & .03 & 0.3 Small \\
& Female & 124 & & 1.22 & .414 & & & \\
\hline B & Male & 152 & $\begin{array}{l}\text { Relevance of knowledge of } \\
\text { emotional abuse to paramedic } \\
\text { practice }\end{array}$ & 274 & 1.39 & .528 & 2.484 & .01 & 0.3 Small \\
& Female & 124 & & 1.24 & .430 & & & \\
& & & & & & & & & \\
\end{tabular}

$* p<0.05$

Respondents were asked to grade their perceived relevance between Highly Relevant (1), Relevant (2), Neither Relevant or Not Relevant (3), Not Relevant (4), Not At All Relevant (5). Section A in Table 15 shows a statistically significant difference $(p=0.03)$ between the way males and females ranked the relevance to their practice of knowledge of physical abuse, and Table $B$ shows a statistically significant difference $(p=0.01)$ between the way males and females ranked the relevance of knowledge of emotional abuse. The effect size however was considered small. 


\section{Table 16}

Independent sample t-test comparing the length of total years of service with respondents' perceived relevance of different types of abuse.

\begin{tabular}{|c|c|c|c|c|c|c|c|c|c|}
\hline Table & Group & $\mathbf{N}$ & Question & $d f$ & Mean & Sd & $\mathbf{T}$ & sig* & Cohen's d \\
\hline \multirow[t]{2}{*}{$A$} & $\begin{array}{l}\text { Length of time in } \\
\text { ambulance service } \\
0-14 \text { years }\end{array}$ & 170 & $\begin{array}{l}\text { Relevance of knowledge of } \\
\text { emotional abuse to } \\
\text { paramedic practice }\end{array}$ & 274 & 1.27 & .459 & 2.239 & 0.03 & 0.3 Small \\
\hline & $\begin{array}{l}\text { Length of time in } \\
\text { ambulance service } \\
14+\text { Years }\end{array}$ & 106 & & & 1.41 & .531 & & & \\
\hline \multirow[t]{2}{*}{ B } & $\begin{array}{l}\text { Length of time in } \\
\text { ambulance service } \\
0-14 \text { years }\end{array}$ & 170 & $\begin{array}{l}\text { Relevance of knowledge of } \\
\text { sexual abuse to paramedic } \\
\text { practice }\end{array}$ & 274 & 1.28 & .462 & 2.253 & 0.03 & 0.3 Small \\
\hline & $\begin{array}{l}\text { Length of time in } \\
\text { ambulance service } \\
14+\text { years }\end{array}$ & 106 & & & 1.42 & .550 & & & \\
\hline \multirow[t]{2}{*}{$\mathrm{C}$} & $\begin{array}{l}\text { Length of time in } \\
\text { ambulance service } \\
0-14 \text { years }\end{array}$ & 170 & $\begin{array}{l}\text { Relevance of knowledge of } \\
\text { neglect to paramedic } \\
\text { practice }\end{array}$ & 274 & 1.24 & .425 & 2.387 & 0.02 & 0.3 Small \\
\hline & $\begin{array}{l}\text { Length of time in } \\
\text { ambulance service } \\
14+\text { years }\end{array}$ & 106 & & & 1.37 & .485 & & & \\
\hline \multirow[t]{2}{*}{ D } & $\begin{array}{l}\text { Length of time in } \\
\text { ambulance service } \\
0-14 \text { years }\end{array}$ & 170 & $\begin{array}{l}\text { Relevance of knowledge of } \\
\text { physical abuse to paramedic } \\
\text { practice }\end{array}$ & 274 & 1.22 & .418 & 2.789 & 0.01 & 0.4 Small \\
\hline & $\begin{array}{l}\text { Length of time in } \\
\text { ambulance service } \\
14+\text { years }\end{array}$ & 106 & & & 1.38 & .487 & & & \\
\hline
\end{tabular}

Respondents were asked to grade their perceived relevance between Highly Relevant (1), Relevant (2), Neither Relevant or Not Relevant (3), Not Relevant (4), Not At All Relevant (5). Section A in Table 16 shows that those paramedics who had served less than 14 years found emotional abuse more relevant to their practice than those who had served 14 years or more $(p=0.03)$. Similarly, section B shows that those paramedics who had served less than 14 years found knowledge of sexual abuse more relevant to their practice than those who had served 14 years or more $(p=0.03)$. This common theme continues with section $C$, which shows a statistically significant difference $(p=0.02)$ between the way paramedics who had 
served less than 14 years thought the knowledge of neglect was more relevant to paramedic practice than those who had served 14 years or more. The effect size, however, was considered small.

\section{Table 17}

Independent sample t-test comparing the years of qualified practice with respondents' perceived relevance of different types of abuse

\begin{tabular}{|c|c|c|c|c|c|c|c|c|c|}
\hline Table & Group & $\mathbf{N}$ & Question & df & Mean & sd & $T$ & sig* & Cohen's d \\
\hline \multirow[t]{2}{*}{ A } & $\begin{array}{l}\text { Qualified as a } \\
\text { paramedic } 0-14 \text { years }\end{array}$ & 224 & $\begin{array}{l}\text { Relevance of } \\
\text { knowledge of sexual } \\
\text { abuse to paramedic } \\
\text { practice }\end{array}$ & 274 & 1.30 & .487 & 2.120 & 0.04 & 0.3 Small \\
\hline & $\begin{array}{l}\text { Qualified as a } \\
\text { paramedic } 14+\text { Years }\end{array}$ & 52 & & & 1.46 & .541 & & & \\
\hline \multirow[t]{2}{*}{ B } & $\begin{array}{l}\text { Qualified as a } \\
\text { paramedic } 0-14 \text { years }\end{array}$ & 224 & $\begin{array}{l}\text { Relevance of } \\
\text { knowledge of neglect to } \\
\text { paramedic practice }\end{array}$ & 274 & 1.26 & .439 & 2.092 & 0.04 & 0.3 Small \\
\hline & $\begin{array}{l}\text { Qualified as a } \\
\text { paramedic } 14+\text { years }\end{array}$ & 52 & & & 1.40 & .495 & & & \\
\hline \multirow[t]{2}{*}{ C } & $\begin{array}{l}\text { Qualified as a } \\
\text { paramedic } 0-14 \text { years }\end{array}$ & 224 & $\begin{array}{l}\text { Relevance of lifespan } \\
\text { and biopsychosocial } \\
\text { developmental } \\
\text { landmarks to } \\
\text { paramedic practice }\end{array}$ & 274 & 1.78 & .758 & 2.579 & 0.01 & 0.4 Small \\
\hline & $\begin{array}{l}\text { Qualified as a } \\
\text { paramedic } 14+\text { years }\end{array}$ & 52 & & & 2.08 & .682 & & & \\
\hline
\end{tabular}

Respondents were asked to grade their perceived relevance between Highly Relevant (1), Relevant (2), Neither Relevant or Not Relevant (3), Not Relevant (4), Not At All Relevant (5).

Section A in Table 17 shows how those who had been qualified less than 14 years perceived knowledge of sexual abuse to be more relevant to their practice than those who had been qualified 14 years or more $(p=0.04)$. Similarly, section B shows how those who had been qualified less than 14 years perceived knowledge of neglect to be more relevant to their practice than those who had been qualified 14 years or more $(p=0.04)$. This theme continued with section $C$, which showed a statistically significant difference $(p=0.01)$ between the way paramedics who had been qualified less than 14 years found knowledge of lifespan development and biopsychosocial developmental landmarks more relevant to their practice then those who had been qualified 14 years or more. The effect size, however, was considered small. 
The quantitative findings identified that paramedics attribute more relevance to the more traditionally understood areas of child protection, such as physical abuse, sexual abuse, and neglect. Different demographics groups attribute different levels of relevance than others, such as those who had been qualified less than 14 years consistently attributing higher levels of relevance. Interestingly, despite emotional abuse being considered a relatively new and difficult to define area of child protection, it was also considered relevant and highly relevant by most. These findings are in contrast to the more socioeconomically based areas of child protection, such as disease prevention and lifespan development, where findings were more varied. However, while statistics can describe what is and what is not, the qualitative investigation can provide a rich narrative of professional practice that illuminates and expands upon numeric data to provide a more holistic overview of the whole study.

It was recognised by all participants that paramedics do have a role to play in child protection. However, there were contrasting views as to how this should manifest in clinical practice. Some participants even voiced annoyance about the amount of responsibility paramedics have. Participants felt that this responsibility resulted from their unique position within the community, but was complicated somewhat by their unease about comparing their standards to those of the families they see, and basing a decision to make a referral upon this. These quantitative findings informed the design of the semi-structured focus groups.

\section{To identify, refer and report: Findings from focus groups}

In all the focus groups, all participants recognised that paramedics play a role in child protection. That role appeared to be undefined by the mention of a formal policy or mission statement, however, and was voiced in various other ways, such as being the 'eyes and ears' for other professions who did not have access to the home environment. This reference was a link to another prominent theme of the paramedics' unique position within the community, which will be explored further. 
[FG3 P2]

The 'eyes and ears' and pick up on things that other people don't, maybe because you see people in an emergency situation.

The reference to 'emergency situation' was a repeated nod to the fact that paramedics can enter homes unquestioned, and how this appeared to define their role somewhat.

[FG3 P3]

We will have that ability to go into those homes and be that really critical eyes and ears in terms of really getting a picture of what is going on for that child. So, our role is absolutely key.

Participants felt that their role as paramedics was primarily a medical one involving assessment and treatment. However, from a CP perspective, they felt that their role was one of acting on behalf of other agencies, such as social services and the police, who would often not be able to see what they had seen. This theme appeared to develop throughout each focus group, and participants began to express their role as an introductory one in a much larger child protection process. This process appeared to be started by the paramedics' ability to recognise signs of concern and then report or refer them to those who would ultimately have a responsibility to investigate and take action. Some participants felt that these roles were easily definable and concise.

\section{[FG4 P1]}

As paramedics, it's not our role to action anything for child protection. It's our role to alert, so our action is to alert agencies that can action and put into place support.

\section{[FG1 P4]}

It's our role to set someone down the right pathway.

Despite paramedics viewing their role as one of working on behalf of other agencies (such as social services), this did not dilute the importance they placed upon their responsibility to 
start this process by reporting and referring their concerns. Some participants alluded to it being their 'duty' to do so.

\section{[FG6 P1]}

Well, we have to report it; it's our duty of care to report.

\section{FG2 P3}

I think you have a duty of care to not only your patients, but also people you come across.

This point is interesting given the variances in relevance placed upon different elements of child protection within the quantitative findings. Some elements were considered less relevant than others, yet there were mentions of a duty of care towards children and young people that would include these elements, such as the recognition of disease prevention. This duty of care appeared to be represented in different ways, and participants appeared to identify a difference between referring and reporting, and their role within this context. The term 'report' tended to be used when cases of abuse and neglect were suspected or perpetrated, and the term 'refer' when there was a clear need to provide a family with a supportive measure.

\section{[FG6 P3]}

The thing is, you're not always referring children, like [NAME] said earlier on, to get the parents into trouble, to have the children taken off them. You're referring them because maybe it hasn't been spotted; they actually need help; they need support.

\section{[FG4 P2]}

They are welfare concerns, as well, so they wouldn't necessarily fall under a category of abuse, but there's still something that needs to be highlighted to another agency that can provide support.

Interestingly, both of the above comments were made from female participants. Female participants were seen within the quantitative data to give higher relevance to knowledge of physical and emotional abuse. Although the absence of a similar comment made by males does not infer that they were not thinking similarly, it does highlight differences. The participants all felt that they had a role to play in child protection. They felt that they were the 'eyes of ears' of other agencies and that it was their responsibility to recognise 
signs of abuse and neglect, and to identify when families required additional support. The difference in opinions, facilitated by the group dynamic, highlighted a wider political debate currently underway in paramedicine, and views on how child protection may be getting caught up in the wider question of the paramedics' job role. Differences in levels of relevance were identified within the quantitative results. However, the qualitative findings identified that participants thought this responsibility could only be fulfilled because of their unique position within the community, as will be explored next.

\section{The paramedics' position within the community and society}

The view that the public see paramedics as having only a medical role (of treatment and transport) was considered an advantage by participants; they thought that this allowed them much greater access to the family home environment than was available to other agencies, who were not routinely or voluntarily invited in. This advantage appears to better allow paramedics to be the 'eyes and ears' of other agencies who were not afforded this access, and it formed part of how they defined their role.

\section{[FG6 P1]}

We're invited in quite willingly by the family of a child. That's why we're there; we've been called there, so already you can see what their environment is like. Whereas, I think other organisations perhaps either struggle to get in, or aren't welcome, or a front is put up almost.

\section{[FG4 P1]}

Because we're invited into that person's house, we're able to see and report a lot more of what's going on, on what's more likely to be a day-to-day basis, rather than a chance to have tidied up.

This seemingly unquestioned invitation into the home environment was seen by participants as possible not only because families required medical treatment, but also because of the perceived perception of paramedics as being trusted. There appeared to be an understanding that because paramedics were not the police and not social services, families trusted them to enter their homes no matter what condition it was in. Some participants 
still thought that some parents would still be hesitant to contact any professional service, including the ambulance service, if they had something to hide.

\section{[FG2 P3]}

They trust us, we're not social services, we're not the police, and a lot of people like that have had those involvements haven't they? They've had the police come.

\section{[FG2 P1]}

If the police turn up, I think they automatically think they're going to take the child away, don't they? Whereas we're seen more as a healer, and we're going to help; we're going to do the right thing, whereas the police can be quite negative, can't they?

The benefits associated with this trusted position were being able to see the home environment in an unprepared way and see how the family, child, or people within the home interact. This was described as the "cleaned up version" [FG1 P1] or indeed the "caught out version" [FG1 P2]. As stated previously, this ability to see the unprepared environment allows paramedics to be the 'eyes and ears' for other services and allows them to notice and recognise issues that need addressing.

\section{[FG3 P1]}

And you'd see them before - if it was, you know, a planned visit from a health visitor or something, they'd have planning, whereas emergency services turn up, and you don't have time to put the vacuum round or clean the kids.

\section{[FG4 P2]}

Because we go in as an emergency situation, they don't have time to hide away their drug paraphernalia or things like that.

Participants felt that parents and families saw paramedics as only being interested in the original reason for the call, and as not having any associated or related issues, and this provided paramedics with an advantageous position within the community, which, in turn, defined their role somewhat. Drug paraphernalia, unexplained bruises, severely neglected personal hygiene, and dangerous living conditions were all things that the paramedic 
participants had witnessed during their practice. While it was recognised that these concerns should be reported to the police or referred to social services, some participants voiced unease about comparing their own personal living standards to those of others, as a means of a cause for concern. This unease and ambiguity appeared to undermine any defined role that had been identified.

\section{Differences in standards}

Although participants recognised that $\mathrm{CP}$ was an important part of their role, it was unclear to some if they were meeting defined thresholds of concern, or just referring or reporting differences in the family's standards of living in comparison to their own. In this respect, one paramedic endeavoured to be non-judgemental:

\section{[FG2 P1]}

Yeah, and because I compare what I do with my children, I very often think, 'Would I do that?' And then, is that right to think would I do that, because my standards might be not be right compared to somebody else's.

Other participants described this unease in practical terms, but participants didn't necessarily agree on what constituted a failure to thrive or neglect:

\section{[FG1 P1]}

And where do you quantify it, 'cause we all know the horrible areas that we go to. Is then acceptable? Is it acceptable for that child to be running around in the same clothes for three days?

\section{[FG1 P4]}

No, no.

[FG1 P2]

They could be dirty but they could be healthy kids, can't they?

\section{[FG1 P1]}

Yeah.

\section{[FG1 P2]}

Cause it's when they're all sunken-eyed and look sort of scrawny, it's different to just a dirty child that's been rolling around in the mud having a whale of a time. 
This was seen in other focus groups to:

\section{[FG2 P1]}

It might all be quite innocent; it comes down to what your standard is, isn't it? Just because somebody doesn't live in a nice clean house, just because they're not clean and a bit smelly, it doesn't necessarily mean there's anything wrong.

This identified theme is an example of how qualitative exploration can expand upon quantitative findings and add different dimensions to objective results. This 'difference in standards' could be seen as a personal 'way of knowing' as described by Carper (1978) and could influence how paramedics come to make decisions. The way paramedics make decisions can infer how they perceive their role in child protection, as will be explored further in the following section.

\section{Ways of knowing}

Categorising paramedics' ways of knowing within Carper's framework can help to identify commonly held perceptions, and see how these influence decisions made within practice and how roles are perceived. It has already been identified that paramedics consider themselves to have a duty of care to be the 'eyes and ears' of other professionals. It must now be considered what types of knowing they use to decide if what they see or hear is of concern to them or not. 
The following questions asked respondents to rate how often they thought they used different ways of knowing when deciding on whether to complete a child protection referral:

\section{Table 18}

Factual knowing (university education, training, signs, and symptoms)

\begin{tabular}{ll}
\hline Perception & Frequency (\%) \\
\hline All the time & $68(24.6)$ \\
Often & $120(43.5)$ \\
Not sure & $64(23.2)$ \\
Not often & $23(8.3)$ \\
Not at all & $1(0.4)$ \\
Total & $276(100)$ \\
\hline
\end{tabular}

Those respondents who responded Not often or Not at all in Table 18 may be those respondents who indicated that they had not had any recent child protection training or education.

\section{Table 19}

Aesthetic knowing (intuition, gut feeling, previous experience)

\begin{tabular}{ll}
\hline Perception & Frequency (\%) \\
\hline All the time & $54(19.6)$ \\
Often & $178(64.5)$ \\
Not sure & $29(10.5)$ \\
Not often & $13(4.7)$ \\
Not at all & $2(0.7)$ \\
Total & $276(100)$ \\
\hline
\end{tabular}


Table 20

Personal knowing (ability to empathise, understand possible prejudices)

\begin{tabular}{ll}
\hline Perception & Frequency (\%) \\
\hline All the time & $29(10.5)$ \\
Often & $143(51.8)$ \\
Not sure & $79(28.6)$ \\
Not often & $20(7.2)$ \\
Not at all & $5(1.8)$ \\
Total & $276(100)$ \\
\hline
\end{tabular}

Table 20 indicates that $28.6 \%$ of respondents were unsure if they used personal knowing or not when making a child protection decision. This could be a lack of understanding in what personal knowing is or a limitation in the questionnaire design.

\section{Table 21}

Ethical way of knowing (what you know and think is right)

\begin{tabular}{ll}
\hline Perception & Frequency (\%) \\
\hline All the time & $29(10.5)$ \\
Often & $132(47.8)$ \\
Not sure & $72(26.1)$ \\
Not often & $36(13.0)$ \\
Not at all & $7(2.5)$ \\
Total & $276(100)$ \\
\hline
\end{tabular}

As seen in the table, $26.1 \%$ of respondents were unsure if they used ethical knowing or not when making a child protection decision. This could be a lack of understanding of what this way of knowing comprises, or could be a limitation in the questionnaire design.

The 'way of knowing' hierarchy paramedics employed when making decisions about child protection referrals was made clear by the quantitative statistics. However, deeper exploration was needed to identify any factors influencing why certain ways of knowing were used more than others. The same open-ended question was asked to participants in each focus group: "What makes you complete a child protection referral?" The overwhelming response was that of 'gut instinct', which then had to be validated by factual 
evidence. It is unsurprising that the perhaps unconscious elements of decision-making and knowing, such as ethical and personal knowing, were vocalised less than what could be felt and experienced during decision-making.

The terms used to describe this 'gut feeling' (aesthetic way of knowing) were numerous and included the following:

\section{[FG4 P1]}

a sixth sense

[FG7 P3]

a deep feeling of unease

\section{[FG2 P1]}

l've just got a feeling something didn't feel comfortable.

Often, these feelings could not be validated in the first instance by the paramedic participants, and could not be fully explained either, but were often described in somatic and visceral terms also.

\section{[FG1 P1]}

I don't know. My gut feeling's saying yes, but why are they high risk? I don't know. I couldn't tell you. I couldn't tell you the reason behind my decision. It was just I feel something is not right here.

\section{[FG3 P4]}

Just something in the back of your mind saying, 'That's not quite right.' It's just - I'm not happy.

Participants were unable to explain what this gut feeling consisted of fully, and did not consider this gut feeling alone a valid enough reason to submit a referral. They felt that 
while it was a good marker of risk, further evidence was required to validate and support it. This suggests a more empirical/factual way of knowing. Participants voiced concern that their referrals would be rejected for lack of substance if their only justification was a gut feeling or intuition. They commented that the original gut feeling or intuition was often a catalyst or a sign that more evidence and investigation was needed for a successful referral, and they often pursued it in the form of investigating physical injuries, hygiene issues, and poor environmental standards.

\section{[FG2 P1]}

But it's articulating that across, isn't it? You can't just write... and I know I have written on the forms; I can't work out what's going on, but something just doesn't feel right.

\section{[FG3 P3]}

You know, your kind of gut feeling. But when you're making your referral, it needs to be factually seen or heard.

[FG6 P2]

So if you go in and say 'something's not quite right here, my gut is telling me', and then you use your observations skills, your listening skills ... But if you've got a gut instinct, you might then look further to find something that is justifiable.

A lack of empirical or objective evidence did not stop all participants from submitting the referral, if all they had to go on was a 'gut feeling'.

[FG 5 P1]

I'd rather do it on a gut feeling and get it wrong, rather than not do it and get it... you know.

\section{[FG4 P2]}

Yes, it has to be, doesn't it? Because sometimes, that's all you've got.

These types of evidence appeared to be more obvious. One participant did mention verbal and situational clues, but there was otherwise little mention of the interactions, body 
language, and non-verbal types of evidence that they would pursue, perhaps because this was likely to be as subjective as the gut feeling.

It was felt that a lack of evidence also alerted participants to the need to complete a referral. A lack of sufficient explanation, for example, was considered suspicious and in need of further assessment.

\section{[FG3 P3]}

So, your inconsistent history. What you're seeing doesn't always appear ...

\section{[FG3 P4]}

I think anything that's unexplained, and can't be explained, perhaps. If they can't explain how something happened, then there's a concern, if they've got a symptom or an injury that's unexplained. Either because they weren't there, or they didn't know, or they can't answer that question. I think anything unexplained would make me concerned.

There was mention of other forms of knowing, but not to the same extent as aesthetic knowing (gut feeling). Participants presented ethical knowing through the mention of caring for their patients and the responsibilities they had toward them.

\section{[FG5 PF1]}

We're all in the job because we care.

\section{[FG4 P3]}

You kind of have a feel that you have an ethical responsibility.

\section{[FG5 PF2]}

We're all in the job because we actually really, really give a shit about what we're doing. 
There was also mention of a personal way of knowing throughout the focus groups more generally, discussing when it would or would not be appropriate to submit a referral based upon what they know or think to be right or wrong, albeit this was mentioned less frequently than aesthetic and empirical ways.

\section{[FG1 P1]}

Or ... it is literally your personal perception. Should we really be judging people on our own standards?

\section{[FG2 P1]}

Yeah, and because I compare what I do with my children, I very often think would I do that? And then, is that right to think would I do that.

By exploring the ways of paramedic knowing, it can be seen why paramedics may submit a child protection referral, but it can also be seen why they might not, and this informs possible improvement strategies. For example, a lack of factual knowing may be due to a lack of training and education, while understanding more about aesthetic knowing might help in finding out what causes discomfort in participants and recognising that they may be intuitively identifying signs of abuse and neglect.

\section{Question 2: How do paramedics perceive the training they receive on child protection?}

Child protection training is a subject that influences more than one area of practice, and similarly affects the answers to more than one of the research questions. This section will include the majority of the general findings related to training; however, if the findings were specifically related to another research question, such as confidence, or barriers to existent practice, they would be included within that distinct element. It has been recognised within the in-depth review of the literature that training for those working with children and young people is key to effective recognition, and the following results and findings evidence how paramedics perceive their $\mathrm{CP}$ training. 


\section{Training and education}

The following question asked respondents to rate their level of agreement with the following statement:

\section{Table 22}

I have received adequate $C P$ training to ensure I can practice safely.

\begin{tabular}{ll}
\hline Perception & Frequency (\%) \\
\hline Strongly agree & $136(49.3)$ \\
Agree & $68(24.6)$ \\
Neither agree or disagree & $66(23.9)$ \\
Disagree & $0(0)$ \\
Strongly disagree & $6(2.2)$ \\
Total & $276(100)$ \\
\hline
\end{tabular}

Table 22 indicates that most respondents agreed to some extent that their training was adequate for them to practise. However, the level to which respondents neither agreed nor disagreed indicates that there may be some elements of training which are better than others. This will be explored further in the qualitative elements.

\section{Table 23}

When was your last $C P$ training update?

\begin{tabular}{ll}
\hline Timeframe & Frequency (\%) \\
\hline Within the last six months & $19(6.9)$ \\
Within the last year & $91(33.0)$ \\
Within the last two years & $105(38.0)$ \\
Within the last three years & $26(9.4)$ \\
Within the last four years & $12(4.3)$ \\
More than four years ago, & $23(8.3)$ \\
Total & $276(100)$ \\
\hline
\end{tabular}


Table 23 indicates that not all respondents had received training as recently as current guidelines stipulate they should (namely, refresher training every three years as a minimum).

The quantitative results indicate that paramedics feel that their child protection training is adequate to prepare them for practice. The majority of those answering the questionnaire had also had training within the last one to three years, making their responses up-to-date and comparable with the most recent training. These quantitative responses, however, contrast dramatically with the qualitative findings and present a different picture altogether.

\section{Do you think paramedics have enough child protection training?: Findings from focus groups}

Participants generally thought that there was a lack of update or refresher training in all aspects of their work, but specifically in child protection. They mentioned that on their year's update training day (if it was not cancelled), child protection was rarely if ever covered, acting as a barrier to effective child protection.

\section{Lack of training}

When asked about the last time they had received child protection training, participants expressed negativity and even concern about the amount they had been given, and voiced concerns over how it was taught.

\section{[FG1 P3]}

I personally can't remember the last time I had training on safeguarding children.

\section{[FG3 P3]}

If you think that people that are trained to do that-social workers - as a job, and that's their job, and they miss stuff, how easy could it be for people that don't get the proper training to miss these things?

Although the consensus was that there was not enough training, there was also uncertainty voiced by some participants who thought the training was adequate but who still wanted 
more. This suggests that those who think training is adequate may still not be completely confident in their knowledge.

\section{[FG1 P1]}

But I think it's difficult to say what's adequate when you don't know what you're gonna be faced with.

\section{[FG2 P2]}

I would say adequate, but I want more, to be honest.

While very few participants felt that they had enough CP training, some participants had differing views in that they were unsure about what else could be taught to paramedics, with references to common sense and experience-based approaches being needed as well as training that was or was not provided.

\section{[FG6 P2]}

Is it not common-sense if we pick up on something that we think is not quite right? Do we need further training?

Some participants however did not know what else could be taught to them as paramedics. This itself created an interesting dynamic within the group and naturally steered the line of questioning towards what would paramedic like to see as training.

\section{[FG1 P1]}

I just keep thinking, what else could we do, though? What else could they give us, trainingwise, for child sex abuse? What ...?

\section{[FG1 P2]}

I don't know.

\section{[FG1 P1]}

I mean, female genital mutilation, they could obviously say, 'These are the ethnic groups to look at. This is what you're looking for,' and that kind of thing, which I don't think any of us have had, but in terms of child sex abuse, like, you get told, the quiet child, the person who shies away, the ... you know, the obvious vaginal trauma in a two-year-old - what's going on? That kind of thing. But what else could they do to prepare you for seeing it? What other training could we have? 
[FG1 P3]

I think having someone that's actually specifically trained in it to come and teach us instead of having a paramedic who probably knows as minimal as we do ... Like, social workers probably don't have ... they probably have specific people that come and train them, don't they, and if they're taking ... if we're saying that we now have a role in it ...

\section{[FG1 P2]}

We should be trained by the same people.

\section{[FG1 P3]}

Which implies we should do ... we should have the training that keeps us up-to-date with all these things.

Interestingly, many of the participants were surprised and even shocked that $73 \%$ of the participants in the quantitative element of this research had said that they felt they had adequate child protection training. This may not have been the case with a larger sample. This difference in feelings within the focus group mirrors that between the quantitative findings and the consensus of the qualitative findings. This may again show that the paramedics either forgot, did not consider, or were not sufficiently aware of these sexual elements of child protection.

Despite the quantitative results indicating that the paramedics thought that they had adequate child protection training, the qualitative consensus was that they had not, and when training was delivered, participants felt that it was not sufficient enough to prepare them for practice, and they expressed criticisms of those who taught these sessions. Participants described the training as basic, happening a long time ago, and not covering as much as they would have liked, such as CSE. Many participants felt that the training they had received had been diluted by its delivery being a small part of more general medicalbased statutory and mandatory education (SME) programmes or as part of online or selfdirected workbooks.

\section{[FG5 PM1]}

It's been, you know, very little training, a very long time ago and the kind of the especially more subtle signs of things, I mean, the kind of non-accidental injuries and things, tend to be fairly obvious, but there's an awful lot goes on, particularly with psychological abuse and 
behavioural clues and things that we easily miss and could make a big difference if we were more aware of them.

There appeared to be recognition that child protection training competed with the various amounts of other training, updates, and refreshers paramedics needed each year, with little time being specifically available for them all to be delivered. Also, elements of child protection had been taught on various training days, such as "radicalization" or "domestic violence" but not on one specific child protection training day, which appeared to be the preferred method.

[FG1 P1]

That's probably'cause it was the Domestic Violence SME ... And that incorporated child abuse.

[FG7 P?]

Even if we've had it, it would have been a half-hour session as part of an SME.

\section{[FG5 PM1]}

I understand that child protection would need to be longer, but it's another one of those pebbles, and eventually what happens is all the water comes out and you're just left with a bucket of pebbles, because there's too much to fit into the day, so the - we only have one day a year and so, to get everything in on one day year is never enough.

What was not provided in face-to-face refresher training days appeared to be incorporated into other forms of delivery, such as online modules or paper-based workbooks. These were met with mixed feelings also, with mentions of time constraints, different types of learning styles, lack of content, and the preference for face-to-face training.

\section{[FG7 P3]}

I think we could do more training. I guess it's just how we have that training because often a lot of our training is online, isn't it? ... Which doesn't really work very well for me. It might work for some people. It depends how you learn, doesn't it? 


\section{[FG3 P3]}

In terms of thinking about 16, 17-year-olds who come under the Capacity Act but also sit within - they are children. And a bit about consent, and understanding that from our perspective in terms of healthcare professionals. You can't cover that in two pages of a workbook.

\section{[FG4 P3]}

I think face-to-face training is important. I think it's far too easy, with the workbooks, to read the question and pull out the answer from that little bit of content around it, and not really think any more about it, and move on.

This element of the focus groups was somewhat limited because the question asked was closed [Do you get enough child protection training?] and answers were often equally as closed, apart perhaps from the preference for face-to-face training. In addition to the emphasis participants placed upon face-to-face training, they also placed much emphasis on experience in the job compensating for the lack of formalised training. Participants used the recent changes in university education to draw a contrast between previous 'on-the-job' training, which provided experience.

\section{Experience-based and university-based learning}

When asked if they had received enough child protection training, participants appeared to refer to experience as being important. The data from this element of the focus groups also contribute to the understanding and exploration of ways of knowing, with experiential knowing being part of aesthetic knowing.

[FG2 P3]

We learn what we learn from each other and from our own experiences.

[FG 5 PF2]

I suppose experienced paramedics would develop that sixth sense, though. 
One participant alluded to using both types of knowledge throughout their career, experimenting with how they utilised taught knowledge in practice depending on any given situation.

\section{[FG 1 P1]}

A lot of it is experimental learning, isn't it, because, as I say, they give you the tools to say, 'This is what these different types of abuse are,' but until you're faced with it, you know, you don't know until you're faced with it, really, do you?

This developmental and experience-based style of practice appears to feature heavily within focus groups, with comparisons being made between experience and training. Participants thought that more experienced paramedics had less training, but that less experienced paramedics had more recent training by virtue of their university route to registration.

\section{[FG5 PM2]}

But if you know, the graduates, none of them have been on the road for more than seven or eight years throughout - across the country.

\section{[FG5 PM1]}

So anyone that, say, graduated two years ago, will only have two years on the road but they will have had far more training at University very recently ... Brand new paramedic straight out of university, no life experience, no job experience, that sort of thing they would doubt themselves, right"

This strand digressed from the original question, but it was considered important. Both participants who thought this way specifically were not university educated and were 16-17year veterans of the ambulance service, which provides a different view, but one that was not overtly obvious within the quantitative findings. Training affects many areas of clinical practice, and a lack of training may result in a lack of confidence. The following section explores this further and presents the findings related to paramedic confidence in child protection. 


\section{Question 3: How confident are paramedics in CP?}

\section{Levels of confidence}

The following questions asked respondents to rate their level of confidence in their ability to recognise signs of different types of abuse and neglect:

\section{Table 24}

Levels of perceived confidence in their ability to recognise signs of physical abuse

\begin{tabular}{ll}
\hline Perception & Frequency (\%) \\
\hline $\begin{array}{l}\text { Very confident } \\
\text { Confident }\end{array}$ & $17(6.1)$ \\
Neither confident or not & $53(198.7)$ \\
confident & \\
Not confident & $8(2.8)$ \\
Not at all confident & $0(0.0)$ \\
Total & $276(100)$ \\
\hline
\end{tabular}

Table 25

Levels of perceived confidence in their ability to recognise signs of emotional abuse

\begin{tabular}{ll}
\hline Perception & Frequency (\%) \\
\hline Very confident & $7(2.5)$ \\
Confident & $121(43.8)$ \\
Neither confident or not & $96(34.7)$ \\
confident & \\
Not confident & $51(18.4$ \\
Not at all confident & $1(0.36)$ \\
Total & $276(100)$ \\
\hline
\end{tabular}

Table 25 indicates that despite emotional abuse being considered within the literature as difficult to recognise, $43.8 \%$ of the respondents felt confident in their ability to recognise it. 
Table 26

Levels of perceived confidence in their ability to recognise signs of neglect

\begin{tabular}{ll}
\hline Perception & Frequency (\%) \\
\hline $\begin{array}{l}\text { Very confident } \\
\text { Confident }\end{array}$ & $23(8.3)$ \\
Neither confident or not & $40(14.5)$ \\
confident & \\
Not confident & $10(3.6)$ \\
Not at all confident & $0(0.0)$ \\
Total & $276(100)$ \\
\hline
\end{tabular}

Table 27

Levels of perceived confidence in their ability to recognise signs of the effects poor housing has on child development

\begin{tabular}{ll}
\hline Perception & Frequency (\%) \\
\hline Very confident & $20(7.2)$ \\
Confident & $156(56.5)$ \\
Neither confident or not & $65(23.5)$ \\
confident & \\
Not confident & $34(12.3)$ \\
Not at all confident & $1(0.36)$ \\
Total & $276(100)$ \\
\hline
\end{tabular}

Despite this socioeconomic element of child protection not being represented well within the paramedic literature, it appears that confidence in its recognition is higher than perhaps might be expected. 


\section{Table 28}

Levels of perceived confidence in their ability to recognise signs of the effects poor levels of social interaction can have on child development

\begin{tabular}{ll}
\hline Perception & Frequency (\%) \\
\hline Very confident & $15(5.4)$ \\
Confident & $147(53.2$ \\
Neither confident or not & $74(26.8)$ \\
confident & \\
Not confident & $38(13.7)$ \\
Not at all confident & $2(0.72)$ \\
Total & $276(100)$ \\
\hline
\end{tabular}

Despite the literature suggesting that paramedic education in this area is sporadic, Table 28 indicates paramedic respondents had a high degree of confidence.

\section{Table 29}

Levels of perceived confidence in their ability to recognise signs of the effects that poor diet has on child development and educational attainment

\begin{tabular}{ll}
\hline Perception & Frequency (\%) \\
\hline Very confident & $29(10.5)$ \\
Confident & $155(56.1)$ \\
Neither confident or not & $64(23.1)$ \\
confident & \\
Not confident & $27(9.7)$ \\
Not at all confident & $1(0.36)$ \\
Total & $276(100)$ \\
\hline
\end{tabular}




\section{Table 30}

Levels of perceived confidence in their ability to recognise signs that a child may not be achieving their expected physical, cognitive, and psychosocial landmarks

\begin{tabular}{ll}
\hline Perception & Frequency (\%) \\
\hline Very confident & $6(2.1)$ \\
Confident & $94(34.0)$ \\
Neither confident or not & $84(30.4)$ \\
confident & \\
Not confident & $85(30.7)$ \\
Not at all confident & $7(2.5)$ \\
Total & $276(100)$ \\
\hline
\end{tabular}

Table 31

Independent sample t-test comparing the current role of respondents with their perceived relevance of different types of abuse

\begin{tabular}{|c|c|c|c|c|c|c|c|c|c|}
\hline Table & Group & $\mathbf{N}$ & Question & df & Mean & sd & $t$ & sig* & Cohen's d \\
\hline \multirow[t]{2}{*}{$A$} & Paramedic & 241 & $\begin{array}{l}\text { Confidence in your ability } \\
\text { to recognise signs of } \\
\text { neglect }\end{array}$ & 274 & 2.10 & .583 & 2.542 & .01 & 0.4 Small \\
\hline & $\begin{array}{l}\text { Advanced } \\
\text { Paramedic (ECP, } \\
\text { CCP, ENP) }\end{array}$ & 35 & & & 2.37 & .646 & & & \\
\hline \multirow[t]{2}{*}{ B } & Paramedic & 241 & $\begin{array}{l}\text { Confidence in your } \\
\text { knowledge of the effects } \\
\text { poor housing have on child } \\
\text { development }\end{array}$ & 274 & 2.38 & .803 & 2.082 & 0.04 & 0.4 Small \\
\hline & $\begin{array}{l}\text { Advanced } \\
\text { Paramedic (ECP, } \\
\text { CCP, ENP) }\end{array}$ & 35 & & & 2.69 & .832 & & & \\
\hline \multirow[t]{2}{*}{ C } & Paramedic & 241 & $\begin{array}{l}\text { Confidence in your } \\
\text { knowledge of the effects } \\
\text { poor diet has on child } \\
\text { development and } \\
\text { educational attainment }\end{array}$ & 274 & 2.29 & .786 & 2.104 & 0.04 & 0.4 Small \\
\hline & $\begin{array}{l}\text { Advanced } \\
\text { Paramedic (ECP, } \\
\text { CCP, ENP) }\end{array}$ & 35 & & & 2.60 & .914 & & & \\
\hline
\end{tabular}


Respondents were asked to grade their perceived self-confidence between Highly Confident (1), Confident (2), Neither Confident or Not Confident (3), Not Confident (4), and Not At All Confident (5). Section A in Table 31 shows how those paramedics without extended skill sets felt more confident in their ability to recognise signs of neglect than did those with extended skill sets $(p=0.01)$. In a similar vein, section B shows how those paramedics without extended skill sets felt more confident in their knowledge of the effects poor housing has on child development than did those with extended skill sets $(p=0.04)$. This confidence in knowledge was seen again in section $C$ where those paramedics without extended skill sets felt more confident in their knowledge of the effects poor diet has on child development and educational attainment than did those without extended skill sets $(p=0.04)$. The effect size, however, was considered small. There was also a significant difference in sample size between those with advanced skill sets over those without.

The quantitative findings surrounding paramedics' confidence levels show a variance in results across most of the subject areas. There are areas in which the literature suggests education is poor and sporadic but about which paramedics feel confident, such as the effects of poor housing, for example. However, the quantitative result surrounding paramedics' confidence levels about sexual elements of CP all indicate very low confidence levels, despite education in these areas being considered in the literature as sufficient and topical.

\section{Table 32}

Levels of perceived confidence in their ability to recognise signs of sexual abuse

\begin{tabular}{ll}
\hline Perception & Frequency (\%) \\
\hline Very confident & $5(1.8)$ \\
Confident & $58(21.0)$ \\
Neither confident or not & $124(44.9)$ \\
confident & \\
Not confident & $81(29.3)$ \\
Not at all confident & $8(2.8)$ \\
Total & $276(100)$ \\
\hline
\end{tabular}

Table 32 clearly shows the wide range of confidence levels about sexual abuse, with fewer respondents overall being confident in its recognition than not. 
Table 33

Levels of perceived confidence in their ability to recognise signs of child sexual exploitation

\begin{tabular}{ll}
\hline Perception & Frequency (\%) \\
\hline Very confident & $4(1.4)$ \\
Confident & $49(17.7)$ \\
Neither confident or not & $118(42.7)$ \\
confident & \\
Not confident & $89(32.2)$ \\
Not at all confident & $16(5.7)$ \\
Total & $276(100)$ \\
\hline
\end{tabular}

Table 33 shows that just $19.1 \%$ of paramedic respondents felt any degree of confidence in recognising CSE despite its now widespread advertisement as a child protection concern. This is a similar finding to that of sexual abuse.

\section{Table 34}

Levels of perceived confidence in their ability to recognise signs of female genital mutilation

\begin{tabular}{ll}
\hline Perception & Frequency (\%) \\
\hline Very confident & $4(1.4)$ \\
Confident & $24(8.6)$ \\
Neither confident or not & $86(31.1)$ \\
confident & \\
Not confident & $98(35.5)$ \\
Not at all confident & $64(23.1)$ \\
Total & $276(100)$ \\
\hline
\end{tabular}

Table 34 indicates the highest reported frequency 'neither confident or not confident' perception of respondents' abilities within this questionnaire, despite it being a legal requirement for HCPs to report suspicions of FGM since 2015. This is a similar finding to that of sexual abuse and child sexual exploitation.

The quantitative results indicated that paramedics were not confident in recognising the signs of sexual elements of abuse; thus, it was explored qualitatively. Multiple reasons became apparent as to why respondents lacked confidence in recognising signs of this kind 
of abuse. Reasons included its hidden nature, the lack of physical symptoms, and an ongoing lack of training.

\section{Lack confidence in recognising signs of sexual abuse, CSE, and FGM: Findings from focus groups.}

The low levels of confidence identified within the quantitative data were mirrored throughout the focus groups. Participants of both sexes and varying ages considered their confidence to be low, especially in comparison with other areas of practice.

[FG 7 P3]

I wouldn't say I was confident at all.

\section{[FG5 PM2]}

There's four of us here, and I'd be surprised if anybody's going to go.... "Yes! Yes!"

Participants felt that because they did not see sexual elements of abuse very often (or so they thought), they would therefore not be confident in recognising it. This presupposes that the participants knew exactly what they were looking for to recognise this abuse, though the quantitative findings suggest this is perhaps not the case.

\section{[FG1 P1]}

I suppose you lack confidence because you don't, hopefully, see it that often ... I think you'd be very unlucky in your career to see a child in child sexual abuse, so therefore, it's not like you do it every day, so you lack confidence in it, I guess.

This view was not voiced solely in one, but was held across different focus group sessions.

\section{[FG4 P3]}

I think we lack confidence in things that we don't do often, and especially something that I imagine people feel is very important. You know, if it was, "Do all your patients have 10 fingers?" We would be very confident that we could manage that, but it's not that... 
In addition to a lack of confidence because of an assumed lack of exposure or a lack of confirmed exposure, participants thought they lacked confidence because sexual abuse, FGM, and CSE is a much-hidden type of abuse. Some participants thought that these types of abuse happen less than physical abuse. They commented that because of the perceived lack of physical symptoms, it was far easier to hide than other forms of abuse or neglect.

\section{[FG4 P2]}

I would perceive that any kind of sexual abuse and things is likely to be very hidden, and behind closed doors, and there's not physical symptoms, as such, is there?

\section{[FG1 P3]}

It's not a case of looking at their arm and noticing a burn mark on their arm. It's questioning and ... well, I mean, you're not gonna examine someone, are you ...

In comparison with normal practice, where someone would be physically examined, participants expressed an avoidance of physical examination. If sexual abuse is suspected or disclosed, this examination is undertaken by a specifically trained forensic doctor, and so this avoidance is correct, and involves working within a scope of practice. This, however, led to paramedics feeling that types of abuse like FGM were more hidden by this process, and in effect, they had been left with one less tool by which they could identify abuse. None of the participants indicated that this process was wrong, but they said that it merely contributed to the hidden nature of this abuse. Paramedics are not in a position or would indeed not want to be in a position to examine or inspect paediatric genitalia unless clinically indicated, for example, in the case of a major trauma.

\section{[FG5 PM1]}

You only examine if it was appropriate to, so if there was an injury to that area, otherwise you're not going to routinely examine. 


\section{[FG6 P2]}

We wouldn't necessarily do a visual check of a child's genitalia unless they were complaining of a specific problem in that area.

These comments highlight the need to be able to focus on the non-physical signs and symptoms of all types of sexual abuse, but specifically FGM. Confidence and / or the need for confidence in areas such as FGM appeared to differ geographically, in that some locations were more likely to receive training in FGM because of a perceived need. Those working in inner-city areas with diverse populations, for example, (while remaining anonymous) were considered to be in need for more training than others. This was recognised by both those working in these areas and those working in more rural locations providing a service to more homogeneous populations.

\section{[FG2 P3]}

I mean [PLACE NAME] isn't particularly diverse, but when you get to jobs in [SECOND PLACE NAME] you do end up in situations with Somali families, or various families, and you think if you've been called there because of severe abdo pain, for example, on a young girl who's just been away on holiday and come back, then yes, that might put up some flags.

\section{[FG3 P3]}

I think, also, for our [LOCATION] crews, being aware of the news story that went on last year where the prosecution of the 13 males for what was clearly CSE - again, it's that increased awareness, and there have been some workshops around CSE and that kind of thing.

Interestingly, neither the quantitative nor the qualitative data suggested that the levels of confidence appeared to increase depending on the kind of area in which participants worked - only that education has been undertaken in these areas. Participants recognised that the type of communities within their geographical location of response (although they could be tasked with responding anywhere in the south-west) was a non-physical consideration of a risk of a particular type of abuse. Other non-physical types of abuse were considered. 


\section{Non-physical signs of sexual abuse}

Participants recognised that due to the signs of sexual abuse and harm being less obvious than those of physical abuse, they had to rely far more on the non-verbal and non-physical signs of sexual abuse, CSE, and FGM. Participants voiced some knowledge of these signs, but still did not feel confident about recognising these sexual elements of practice, and so remained ambiguous about them.

[FG3 P2]

Because, quite often, sexual abuse will only come to light with disclosure, and children and young people will be really reluctant to disclose because of the stigma, because of the concern that they won't be believed, and so on and so forth.

[FG2 P1]

It puts more onus on the interactions, body language, the less black and white signs.

Participants noted the need to look for non-physical signs, but also recognised that there may be many different reasons for concerning or abnormal behaviours, although no triage or escalation process for these signs was mentioned. Other reasons for these signs could be having a stranger in their home, normal family discourse, recurrent urinary tract infections causing soreness, and different cultural normalities.

\section{[FG2 P3]}

Well, I like to believe that I would be perceptive enough to be in a situation to say 'Something is wrong here. This person is not comfortable; there's a dynamic here that isn't working'. Whether I would go immediately to 'This is a sexual abuse situation', as opposed to

'This child had been bullied at school', or that something else was wrong, I don't know.

\section{[FG3 P1]}

I wouldn't recognise if a child is sexually abused. All I would understand is maybe there's not the interaction with a parent or some member of the family that you would expect them to have interaction with. What are the causes of that? It can be caused by many things. 
The uncertainty surrounding these non-physical signs and behaviours was evident throughout the focus groups, as was the uncertainty around any escalation process of anything that cannot be proved. It is clear that there is a discord between the more generic elements of child protection practice and the sexual elements, chiefly in normal levels of expected confidence. There may be multiple reasons for and barriers to paramedics feeling confident and being competent in sexual areas of child protection practice, one of which could be training, as will be explored next.

\section{Lack of training in sexual abuse, FGM, and CSE}

Lack of training was a prominent theme surrounding paramedics' confidence levels regarding the sexual elements of $\mathrm{CP}$.

\section{[FG3 P3]}

It comes back to training again, doesn't it? In terms of equipping us, as healthcare professionals, in knowing what to look for in terms of signs of CSE, sexual abuse, FGM, giving us that additional knowledge to be looking for those signs and symptoms, the concerning things that we need to be worrying about.

\section{[FG4 P1]}

We haven't had the training to recognise the non-verbal signs and symptoms of sexual abuse, and to pick up on the other evidence around people's behaviours; how their interactions are with other people within the room, especially children and their parents, and other adults or other children.

Lack of training was a common theme and was not confined solely to the sexual elements of child protection. Given the low levels of confidence highlighted within the quantitative element, it seems unsurprising that the views surrounding training appeared negative.

Not all the participants agreed with this view, however, and one participant thought that they were in fact adequately trained and that staff had a responsibility to self-direct their own training and thus, did not require any more. This view was held until the group began to discuss sexual aspects of child protection, and through this dynamic, challenged the concept of adequate training. 


\section{[FG6 P2]}

I think we've had adequate information in the past; there's information about safeguarding on all the computers. We are a profession; it is up to us to agree to read up on things, and look at things, and to make sure that we're up to date with what's going on. And I think I know enough that I wouldn't need any further training on safeguarding.

\section{[FG6 Facilitator]}

So under the Serious Crime Act 2015, paramedics are now legally obliged to report their concerns, and report suspicions of female genetalia mutilation. Do you think now that legal obligation that paramedics have marries up with the training that we've had?

\section{[FG6 All] [Disagree]}

\section{[FG6 P2]}

I think the stats speak for themselves.

\section{[FG6 P3]}

I didn't know about that, not at all.

[FG6 Facilitator]

So you weren't aware that...?

[FG6 P3]

We were legally bound, no.

\section{[FG6 P2]}

So now our training is inadequate isn't it?

\section{[FG6 P3]}

We can't read everything, see everything, we don't know everything, all these things are changing all the time. But no, that's definitely not; and that would be good to be taught that really.

This lack of training may be a barrier to effective child protection, a subject area which we continue to explore.

\section{Question 4: What barriers exist to paramedics undertaking their role in child protection?}

This findings chapter has already highlighted certain barriers to paramedic child protection practice, such as the lack of training and poor confidence levels. This element will continue to present findings which show the barriers paramedics thought existed within their current practice. 


\section{Lack of feedback}

It was highlighted within both the quantitative and qualitative results that a lack of feedback of child protection referrals was considered a barrier, and possibly contributed to other barriers, such as the feeling of not being taken seriously. The following table indicates the frequency of referrals made by respondents and shows that they could comment on the area of feedback with validity.

\section{Table 35}

Within the last three years about how many child protection referrals have you made?

\begin{tabular}{ll}
\hline Estimated amount & Frequency (\%) \\
\hline$\leq 10$ & $250(90.6)$ \\
$10-20$ & $21(7.6)$ \\
$21-29$ & $3(1.1)$ \\
$\geq 30$ & $2(0.7)$ \\
Total & $276(100)$ \\
\hline
\end{tabular}

Table 35 indicates that most of the respondents had submitted fewer than ten referrals each year, which anecdotally appears consistent with normal practice.

The following question asked respondents to rate their level of agreement with the statement about feedback from referrals they had made:

Table 36

I regularly get feedback from the child protection referrals I have submitted.

\begin{tabular}{ll}
\hline Perception & Frequency (\%) \\
\hline Strongly Agree & $0(0 \%)$ \\
Agree & $33(11.9)$ \\
Neither agree or disagree & $62(22.4)$ \\
Disagree & $98(35.5)$ \\
Strongly disagree & $83(30.0)$ \\
Total & $276(100)$
\end{tabular}

Table 36 shows that $22.4 \%$ of respondents neither agreed nor disagreed, which may indicate that the amount of feedback they receive changes. 
The quantitative findings were also mirrored within the focus groups. Lack of feedback was shared as a common theme and was considered a barrier to learning. Respondents felt that not only would it be nice to find out what had happened to their patient, but that the validation or improvement of referrals could also take place and instil confidence.

\section{[FG1 P1]}

It would also help if you got feedback, I think ... A lot of the times you submit them without feedback, don't you, and you wanna know what happens. Sometimes you get feedback, but not a lot of it.

\section{[FG5 PM2]}

You do a referral, and you have absolutely no idea that it's been picked up on the other end.

Paramedics recognised that feedback was a learning opportunity; they wanted feedback so they could improve the quality or choice of their referrals. They also welcomed criticism from their referrals and noted that it was a way of improving future recognition and referral.

\section{[FG1 P1]}

If you got feedback to say, you know, 'Did you pick up any of these signs when you went out?' then you might think, 'Oh, actually, thinking about it, maybe there were one or two little signs that l've obviously completely swept under the carpet and missed,' but now, knowing about that, you've learned from it, haven't you?

\section{[FG3 P3]}

Feedback is really important, because it gives you that kind of reassurance that you did do the right thing; if you didn't do the right thing, how to make it better next time. I think feedback is really important.

This lack of feedback may contribute to a feeling that paramedic staff are not taken seriously, which was also explored as a barrier to effective CP. 


\section{Being taken seriously}

Respondents were then asked to rate their level of agreement with the statement about being taken seriously:

Table 37

I feel my child protection referrals are taken seriously by the receiving department.

\begin{tabular}{ll}
\hline Perception & Frequency (\%) \\
\hline Strongly Agree & $10(3.6)$ \\
Agree & $101(36.5)$ \\
Neither agree or disagree & $129(46.7)$ \\
Disagree & $29(10.5)$ \\
Strongly disagree & $7(2.5)$ \\
Total & $276(100)$ \\
\hline
\end{tabular}

It is not possible to comprehensively answer this question without speaking to outside agencies. However, given that earlier results show paramedics view their role as one of being the 'eyes and ears' for other agencies, it was important to explore further whether they felt they were taken seriously. This may also influence whether paramedics submit child protection referrals or not. Participants expressed mixed feelings depending on their experience, with some relying on assumptions that they were taken seriously because of the lack of feedback from their referrals by receiving agencies.

\section{[FG5 PM2]}

We can only assume so because we don't - we don't, we never find out.

\section{[FG3 P3]}

Not as much as they could do, but I think, historically, we have not been seen as particularly important, but I think moving forward, we are being recognised as a more valuable resource in terms of the child protection world.

Amongst these mixed feelings, there was a recognition that perhaps agencies were starting to consider more seriously the role paramedics play. Some participants felt strongly that 
they were taken seriously and that this was evident by their inclusion in serious case reviews, although participant inclusion appeared to differ geographically, with some participants saying that they were never invited. The group dynamic of the focus groups allowed two viewpoints to be shared simultaneously.

\section{[FG7 P3]}

I think we're taken seriously, yeah. Like when I've been to a child death review or something like that, they want us to be there.

\section{[FG3 P1]}

I don't know. We were invited more to case reviews, and stuff like that. We never used to do; that's started now.

The question that arises is whether if more feedback from referrals were given, staff would feel as if they were taken more seriously. Another query is whether this lack of perceived support and respect stops paramedics completing child protection referrals. This and other reasons are considered more specifically within the next table. 


\section{Reasons for not submitting child protection referrals}

Some of the reasons for not submitting child protection referrals have already been highlighted within this chapter, such as a lack of training, for example. The following questions asked participants directly if they recalled times where referrals had not been made, and why.

\section{Table 38}

On reflection, with hindsight, and with experience, I recall times where child protection referrals should have been completed but were not.

\begin{tabular}{ll}
\hline Perception & Frequency (\%) \\
\hline Agree & $150(54.3)$ \\
Disagree & $126(45.7)$ \\
Total & $276(100)$ \\
\hline
\end{tabular}

Table 38 indicates that despite child protection referrals needing to be submitted, there have been cases where they have not been. 


\section{Table 39}

Please indicate any reasons as to why child protection referrals may not have been completed in practice

\begin{tabular}{lccc}
\hline $\begin{array}{c}\text { Reasons why referrals may not have } \\
\text { been completed }\end{array}$ & Frequency & $\begin{array}{c}\text { Percentage of } \\
\text { all responses }\end{array}$ & $\begin{array}{c}\text { Percentage of respondents } \\
\text { citing this reason }\end{array}$ \\
\hline Suspicion but no proof & 120 & 15.1 & 43.5 \\
Not stood down & 110 & 13.8 & 39.9 \\
Not enough time & 108 & 13.6 & 39.1 \\
No options apply & 73 & 9.2 & 26.4 \\
Poor facilities & 68 & 8.2 & 24.4 \\
Realised too late & 65 & 8.2 & 23.6 \\
Lack of confidence & 63 & 7.9 & 22.8 \\
Afraid if outcome wrong & 56 & 7.0 & 20.3 \\
Never get any feedback & 40 & 5.0 & 14.5 \\
Poor knowledge of abuse and neglect & 35 & 4.4 & 12.7 \\
Paramedic referrals not taken seriously & 21 & 2.6 & 7.6 \\
Felt intimidated by parents & 16 & 2.0 & 5.8 \\
Wanted to avoid confrontation & 13 & 1.6 & 4.7 \\
I only have to report abuse and neglect & 4 & 0.5 & 1.4 \\
Not my responsibility & 3 & 0.4 & 1.1 \\
\hline
\end{tabular}

Table 39 indicates the extent to which the respondents identified with the reasons offered by the researcher. The option 'not stood down' refers to the standing down of an ambulance crew; when an ambulance crew is 'stood down', they are made unavailable for emergency calls. There are various reasons why this would happen, for example, cleaning a vehicle, writing a police statement, or a meal break.

The option of 'no options apply' was selected 73 times by respondents. It was considered possible that respondents may not have identified with all or any of the reasons offered by the researcher, and so a free-text option was also provided. Interestingly, only two further themes were identified, which were 'before reporting mechanisms were in place $(n=2)$ and verbal handover was made $(n=2)$.

It is clear that there are many, often complicated, reasons as to why paramedics recognised that referrals had not been made and that exploring them further could provide a more indepth understanding of the issues. 


\section{Lack of time or permission}

Time was a prominent theme in why referrals were not made. Paramedics did not have time to complete the referrals during the 15-minute handover period, and requests to dispatchers to be 'stood down' from active duty were refused due to high demand and excessive workload.

[FG2 P1]

I think time, isn't it? You don't get stood down, and obviously, I've not used the new thing, so I can only talk from before, but you'd make notes and think, 'That one needs safeguarding', then you're back an hour later on station; can I safeguard it... how many people are going to stay and do it?

\section{[FG6 P2]}

The last thing you want to do, and I don't care what anyone says, at three o'clock in the morning after you've been run ragged, you come down to the station and the first thing you want to do is have a cup of coffee or something, sit down. You sit down, and then you think, "Right, I've got to do that referral", and if it's one of those that's 50/50, are you going to think, "Oh they'll be alright. I'll have my coffee and put my feet up." If it is a blatant obvious case of some sort of abuse, then I would hope everybody would say "Sod the coffee; I'll do this referral". But it's those 50/50 ones I think you're going to potentially miss, because maybe people don't feel as though they've got the time, inclination, motivation.

\section{[FG5 PM2]}

So if you can imagine that maybe the third job in the morning is $10 o^{\prime}$ clock in the morning and you and it's a child that you think that you might - oh I don't know, whatever - you think you've made your mind up, and you're already on your next job and your next job after that, then you'll get your break. You're not going to do it on your break, in your break window because it's the only time you'll get to eat. So you scoff your food down - 31 minutes later, you're back out doing the next job.....

The implications of time, workload, and in some cases, motivation appear to be evident here, in that referrals are not being made. There appears to be a balancing act between 
completing referrals that may not overtly need submitting versus eating lunch and being able to have a drink, which is important in a 12-hour shift of physical work.

Relationship with dispatch was also a contributory factor in referrals not being made. Paramedics stated that dispatchers did not understand the importance of these legal documents and the reason they are needed.

\section{[FG1 P1]}

I think you would have to tell Control. I mean, they are meant to, if the child's high-risk or an adult's high-risk, they are meant to stand you down.

\section{[FG3 P3]}

You know, "Can I be stood down to do a child protection referral?" - "No, we've got outstanding emergencies." You'll do it later, and by the time you're doing it, it's the end of your shift. And do you know what? We've just done 13, 14 hours.

There appears again to be this balancing act between completing referrals that may not overtly need submitting and responding to other emergency calls which are waiting. Competing priorities appear to be acting as a barrier to effective child protection referrals being made. In addition, when there was time for a referral to be completed, staff appeared to be faced with software or computer issues, as discussed next.

\section{Technology difficulty}

Frustration was felt at the difficulty of completing the forms themselves. At the time of this research, child protection referrals were done using a desktop computer at an ambulance station, something which we already know the paramedics found difficult to in use due to time constraints. When they were 'stood down', they found the online forms themselves were unfit for purpose. 
[FG3 P3]

The reporting mechanism, in terms of the retrospective, when you're back in the station filling out a form - it's not fit-for-purpose.

The difficulties and frustrations reported by participants appeared to be simple, such as error boxes reporting insignificant or inapplicable boxes had not been ticked or written in.

\section{[FG1 P2]}

The form that's on there at the moment, you're just like, you think you've written whatever in the right bit, and then you're like, "Oh, did they not want that there?" It's just a silly form ... And it's not conducive to being helpful.

\section{[FG1 P4]}

It's infuriating ... It just keeps returning you to minor errors ...

Participants showed ways of working around the laborious system of completing the forms by ticking various boxes and just completing a free-text box with all the information they deemed appropriate. These were issues which participants found frustrating and which may have stopped them completing child protection referrals unless there was an emergency or obvious case of abuse or neglect. Some of these issues were also being negated by the Electronic Patient Care Record (ePCR), which will be explored further.

\section{Parental consent}

Paramedics felt concerned that if parental consent was not gained, it meant (in nonemergency cases) that no progress could be made on the referral. This appeared to demotivate paramedics from completing referrals. Allusions were made to how asking for parental consent can often result in a confrontation process, which meant some paramedics avoided such situations. 
[FG3 P3]

Because if you submit a child protection referral, if the concern is not at the point where it's child protection, so you're just thinking about a child in need, if you don't have consent from the parents to make that referral, it won't be looked at.

[FG3 P2]

And if they don't get the consent, then it's a waste of time anyway. It's the ways of asking for the consent, without being like, "Oh, yeah, do you remind if I report you to social services?" It's where you're sort of saying, "Oh, would you like some help?" We could maybe.

\section{[FG4 P1]}

I think it's because it's not something that people do all the time. I don't think crews are confident in having a conversation with patients about doing a referral, because I think crews' perceptions, and the general public's perceptions - as soon as you say the word 'safeguarding', you think, "My granddad's going to be put into a care home, and my kids are going to be taken away from me".

There appeared to be confusion surrounding this aspect of referral completion. Was consent needed or not? This may have been clearer or less confusing had those who made referrals got more feedback from outside agencies. This is just one of many suggestions that were generated throughout the focus groups, and this leads on to the next element of this chapter - improvements.

\section{Question 5: Can any ways of improving paramedic's practice in child protection be identified?}

Throughout the focus groups, it became more obvious that participants were not sharing solely their feelings, thoughts, and frustrations about current practice, but were also sharing valid and insightful ideas as to how they thought practice might be improved or was improving.

\section{Electronic Patient Clinical Record}

The ePCR is a tablet-based clinical record form, which also has the child protection referral form built into it. With its use of wireless internet, it can be used to submit a referral 
without the need to find the time to go back to a desktop computer on an ambulance station. The ePCR appears to have been accepted as a method of completing referrals, although this was only considered from a child protection perspective. At the time of enquiry, the ePCR was not standard across all areas of the sample ambulance service or ambulance services nationally.

\section{[FG2 P3]}

It auto-populates most of the information. There's a safeguarding tab in which you'll add that additional information that would have been on the safeguarding form ... It will autopopulate the job number, the patient details into a referral form, and then you just send it to safeguarding. So as part of what you were already doing, you're not having to type out their address, again .... That process has made it easier to do...

\section{[FG2 P3]}

Whereas that's the good thing about the ePCR; it's within your time with patient, so you do it before you clear, until it's done.

\section{[FG5 PF2]}

And if it's part of your ePCR whether you're still on scene or up on A\&E you're completing an ePCR, so it's not going to take you a lot longer; you'll just deal with it then and there...

The ePCR system appears to prompt the user to think about completing a CP referral. This is done by virtue of the accessibility it provides, not a computerised message prompt, and the users who made a comment during the focus groups considered this an advantage.

\section{[FG5 PM2]}

The EPCR does have more prompts for, I say, is it behavioural? Is it reasons - is the reason you're safeguarding, is it because of behavioural signs? Is it because of physical signs? Is it because of disclosure by the patient or someone else? That sort of thing; so it's better on ePCR. 
Despite the completion of referrals now seemingly being eased by the new and easier ePCR, the paramedic is still required to know when a referral should be made. For this to happen, participants thought that different styles of training were needed than are currently in use.

\section{Scenario-based training delivered by experts outside the ambulance service}

Those participants who thought more training was needed expressed a preference for it to be delivered by experts from outside the ambulance service, such as professionals from child and family social care services, health visitors, and paediatricians. This was apparent in all the focus groups. Specific significance was placed upon scenario-based training, something paramedics are historically used to as part of various forms of education and training.

[FG2 P3]

We get trained in safeguarding by paramedics now; in some ways, it would be more useful to be trained in safeguarding by social workers, definitely.

\section{[FG 4 P2]}

I think, as well, maybe it should be from somebody with expert skills as opposed to just the training team in-house.

Participants felt that these experts in the field could tell them exactly what it was that they wanted them to look out for, and felt that they should know the entire process from referral to the assessment and the outcome. 


\section{[FG 5 PF1]}

Because we know our process, but what happens when it's given to social services? What is their process? You know, we see something awful, we - maybe nothing's done about it.

\section{[FG7 P?]}

"It would be nice to know various case studies, how the actual safeguarding process worked all the way through from when we actually made the referral into what actually happened step by step".

Participants felt that while being taught by experts, they would learn more about safe practice and that the experts were better able to answer questions than the learningdevelopment tutor paramedics.

\section{[FG2 P3]}

I'm sure there are things like that with safeguarding from a social services point of view; they go, "You were in that situation, and you had that opportunity to do $x-y$, or ask $x-y$ and you didn't? Why didn't you do that?" And you think, "Ohh, because we had no idea it would be relevant to the future course of what would support those people".

\section{[FG5 PF2]}

It would be interesting to see, well, we deem that a high risk, medium risk, and low risk because of these factors and these factors and these factors, and then we would know where we stand in each of those cases.

Scenario-based training and education are also not unusual, especially in paramedicine. Most paramedics are likely to be au fait with objective structured clinical examinations (OSCE) as part of both their original training and education as well as their refresher days. Participants specifically voiced a preference for expert delivered training, which was scenario or case-based. 


\section{[FG1 P1]}

Have a case scenario where real instances have happened involving ambulance staff that you can look through and digest ... Yeah, so a case scenario rather than just saying, "This is physical abuse; this is financial abuse..."

\section{[FG7 P3]}

I mean, it would be good to have kind of case studies, wouldn't it, on things? Things based on kind of... I guess, you can't use real life, but things based on examples of what's gone right and what's gone wrong.

This type of scenario-based learning was important to participants, with those who did not voice a specific preference agreeing when it was mentioned within the group (Focus Group Notes). Such scenario or case-based training may provide an opportunity for participants to use their experience to approach learning rather than just a theoretical one. This experience-based type of learning also emerged as a theme, and it was thought to be facilitated by peer support.

\section{Peer review and colleague support}

Participants felt that peer review and support was important, especially given the increases in workload and decreases in quiet time. Previous years of service have seen fewer emergency calls and more time on ambulance stations with colleagues discussing and informally reflecting upon cases, mistakes, and training.

\section{[FG2 P1]}

I think a lot of it comes down to chatting to your peers; "I've just been to this job, a bit weird. I'm going to fill out a safeguarding, what do you reckon?" And somebody will say, "Oh no, don't be daft, your standards are too high", or "That actually does sound a bit... yeah, yeah". 


\section{[FG3 P1]}

So, sometimes, when you leave and you talk about a case with your crewmate afterwards, you say, "What did you think of that?" And they say, "Well, actually, I was a bit worried about that." ... Discussions afterwards, and we do that as crews, don't we?

Some but not all of these examples relied on going back to a station and having the time to discuss cases with other colleagues; however, as seen in earlier discussions about the ability to complete online child protection referrals, this was getting more difficult to do. Some participants felt that the 'mess room chat' was being diminished, and with it, some learning.

\section{[FG6 P2]}

Maybe we did once upon a time, but it doesn't happen quite so much now because very rarely are you on station, and even more rarely are there two vehicles on station at the same time. So you tend to do it maybe at the beginning/end of shifts, that swap over time. Or even outside A\&E, you might see somebody that you'll think you'll have a quick chat with them; you go over and speak to them: 'I've just been to this job, I did 'this - what do you think?".

\section{FG4 P2}

"It could be down to discussing it with a colleague afterwards, and them going, "Well, I would have done."

This theme of peer support or review was prominent throughout the focus groups and did not present specifically through one question, but rather through discussions of various elements. Clinical supervision is now a popular and validated method used within clinical practice to reflect upon clinical practice to learn from mistakes and challenges. This informal type of clinical supervision appears to have been eroded by an increase in demand. 


\section{Summary of findings}

The results and findings section has presented UK paramedics' views, thoughts, and perceptions regarding their role in child protection using a mixed methods approach. It has explored their perception of child protection training, the perceived barriers to effective practice, and importantly, it has examined how they think improvements can be made. The quantitative methods provided a foundation of information upon which the focus group questions could be based while the descriptive statistics highlighted areas which warranted further exploration using the focus groups. This qualitative method provided deeper meanings and gave a personal, professional context to the presented statistics. This sequential method will contribute to the research questions being answered.

What has become clear throughout this chapter is that most paramedics viewed their role in child protection as being the 'eyes and ears' for other professionals who were not granted access to the home environment. This access was seen as an advantage over other professions and was used to those who paramedics thought were the experts in protecting children. Paramedics thought that this proxy role was one of information gathering, referral, and reporting, rather than full investigation. They most frequently used an aesthetic way of knowing, which was described as a 'gut feeling', and they used this feeling as a cue to purposely gather factual and objective evidence to be presented in a referral or report, thus, using an empiric way of knowing. At times, ethical and personal ways of knowing were used to appease the internal conflict surrounding the differences participants had in personal living, dietary, and disciplinary standards of parenting with their patients. Many participants found it difficult to know when a threshold of concern was actually met. This difficulty might result from a lack of training and education in child protection. Interestingly, when questioned quantitatively, most of the paramedic participants thought that they had adequate child protection training to practise, with only a minority not having had training within the last three years. Qualitatively, however, the paramedics voiced concern over the lack of training they received and regarding the lack of external expert involvement and were surprised by the quantitative results. Feedback was considered as a method of training 
that was very helpful for future practice; however, views differed both quantitatively and qualitatively regarding the amount of feedback paramedics received. This may represent a difference in questionnaire respondents and focus group participants, but also may represent the difference in data collection methods. The benefits of the focus group environment included having an open discussion about what feedback meant to the paramedics and how it is delivered, something which was not offered within the quantitative questionnaire. This may mean that some paramedics receive different types and different amounts of feedback. There was little consensus as to whether paramedic referrals are taken seriously by receiving departments, which might have resulted from sporadic and inconsistent frequency of feedback. Despite this, however, most of the paramedics questioned considered all the key elements of child protection to be relevant to their practice, with physical, emotional, and sexual abuse being considered slightly more relevant than the psychosocial aspects of practice. The levels of relevance applied to paramedic practice by those questioned tended to mirror their confidence levels, apart from sexual types of abuse, where there was a significant contrast. Participants were not confident in their ability to recognise the sexual elements of child protection due to a lack of training, time on scene, and the very hidden nature of sexual abuse, which made it difficult for paramedics to recognise it. As such, their confidence was low. Low confidence was one barrier to effective child protection, but others included a lack of time to complete referrals, a lack of peer support, a lack of training, and online referral forms which were not fit for purpose. It was thought that child protection would be improved by changes being made to these elements of practice, such as the introduction of ePCRs, and scenario-based training provided by outside child protection experts. The results and findings in this chapter, which were gathered sequentially, have provided rich and contextually in-depth data about paramedic-related child protection. The data now need to be analysed to contribute further to the overall aims of this research, chiefly, to inform future paramedic practice, policy, and education. 


\section{Chapter Five: Discussion}

This chapter will discuss the research results and findings with the aim of informing future paramedic practice, policy, and education. The qualitative study has added colour, shade, and nuance to the quantitative investigation and enabled a more holistic overview of the whole study, which will be discussed here. At this point, it is worth remembering the research questions used to attempt to achieve these aims:

1. How do paramedics perceive their role in child protection?

2. How do paramedics perceive the training they receive on child protection?

3. How confident are paramedics in child protection?

4. What barriers exist to paramedics undertaking their role in child protection?

5. Can any ways of improving paramedic's practice in child protection be identified?

These results and findings will be discussed in the context of the most up-to-date recommendations at the time of writing from various sources. Such sources include the Royal College of Paediatrics and Child Health (RCPCH) (2014) Safeguarding Children and Young People: Roles and Competences for Healthcare Staff, Intercollegiate Document. This document, for example, sets out the roles and responsibilities of HCPs and prescribes the minimum training requirements needed to fulfil these roles. It was written with input from 21 organisations, including the National Ambulance Safeguarding Group, and can be used to contextualise the analysis further. The elements of practice that paramedics were questioned about within this research were all considered integral parts of modern day child protection. There has been a small amount research investigating the perceived relevance of subjects to individual performance, albeit in cooperative education program students. Those who see a greater degree of connection between the work and the tasks they are carrying out are more likely to feel satisfied and engaged, and in turn, are more likely to perform well (Drewery et al., 2016). It was thought that the quantitative measure of 
relevance paramedics placed upon these elements of practice could be used as an indicator to identify the perception of their role, and thus it was explored qualitatively.

\section{Research Question 1 - How do paramedics perceive their role in child protection?}

Paramedics predominantly perceived their role as a proxy for other more suitably qualified professionals, whose main job it is to safeguard the children in question. Paramedics saw themselves as being the 'eyes and ears' for other professionals, and considered that their role was to gather information and evidence, but not to investigate any further. This perception is supported by JRCALC (2017) guidance, which states that the role of the ambulance service is not to investigate suspicions, but to ensure that any suspicion is passed to the appropriate agency. This guidance is limited, however, as without further investigation, however minor, many paramedics would feel unable to refer their concerns, and feel the need to ensure that there is a reasonable ground to suspect a serious level of concern, as stated within NICE (2013 p9) guidance.

The perception of this role was conflated further by the type of contact the paramedics had with such families. For example, if the child were extremely physically sick or injured, there would not be enough time to assess for child protection issues within the home, as the priority would be to stabilise the child and get it to definitive medical care. Yet even the limited time spent in a child's home would often be more than other professionals would be able to achieve unless there had been a crime reported. This unique position within the community was recognised as an important element that allowed the paramedics to undertake their proxy role. Paramedics thought that families did not view them as social services or as the police, and so they were more likely to be invited into an environment from which other agencies would be excluded. Participants voiced concern that too much overt investigation on their part would deter parents from calling for emergency help in the future, whether their children needed it or not. This perception further defined their role as being the 'eyes and ears' for other professions, and was portrayed by participants as being a protective and subtle one. 
Given the scope of practice and expertise of UK paramedics, this was not viewed by the researcher as a negative point per se, but was viewed rather as a reasonable perception. Paramedics are specialists in urgent and emergency care, and often consider themselves to be a very short-term solution to longer-term issues. Paramedics frequently treat people within the community without the need for specialist care, such as falls, minor injuries, and respiratory distress. Patients, however, who require more specialist expertise and equipment are taken to hospitals where this can be provided; this is no different for children and young people who require specialist social and family care and support. Paramedics provide the evidence required to support this referral or report to specialist social care.

There remains a lack of data regarding how many children paramedics encounter in daily practice and how frequently they are involved in child protection processes, something which may affect the way they view their role. This research has shown that $90.6 \%(n=250)$ of the paramedics had submitted fewer than 10 referrals in the last 3 years. However, this question relied on the memory of the paramedics and does not account for referrals which should have been made but were not. The introduction of new technology and new referral pathways may also now mean that this data can only be contextualised to a certain geographical area of practice at a certain time; however, the data still suggest low levels of referrals by paramedics. The in-depth review of the literature suggests that paramedics have a key role to play despite the lack of frequency data, and this is evident in the eyes and ears concept. Participants felt that had an eyes and ears role and only spoke about frequency in terms of the amount of exposure they had to the various types of abuse victims they encountered. They considered the lack of exposure to be a negative point and felt it impeded their ability to recognise abuse.

With the term 'eyes and ears' having been recognised, it was questioned what it was that paramedics were actually looking and listening for, and how they were alerted to having to look and listen. Historically, in ambulance service training, more emphasis has been placed upon subjects such as sexual abuse, physical abuse, and neglect. The researcher thought that there might be far more relevance placed upon the historically emphasised aspects of child protection practice as opposed to some of the more psychosocial aspects of care; 
however, this was not the case. There were only small differences in the levels of relevance placed upon physical abuse, for example, $(100 \%)$ in comparison with psychosocial elements of child and family health (91\%) and lifespan development (85\%). Paramedics perceived both areas as relevant to their role in child protection. Some areas were considered 'not relevant' and 'not at all relevant' by some respondents, albeit only a few. Health promotion and disease prevention, for example, was considered 'not at all relevant' by $0.7 \%(n=2)$ of respondents, whereas the relevance of lifespan development was considered as 'not relevant' by $3.3 \%(n=9)$ of respondents. These generally positive findings may represent the changing face of child protection training and education for paramedics internally and at the university level. Not only does this highlight that they perceive themselves as having a role in child protection, but it also shows that this role is far more complex than previously thought and is inexorably linked to socioeconomics, social welfare, and education, rather than solely being about physical abuse and neglect.

Being the 'eyes and ears' for other professionals might be seen as a reductionist approach to child protection. However, the participants felt that their responsibility in this matter was nevertheless an important one. This perceived role was not born out of laziness or a lack of alacrity, but rather stemmed from a lack of expertise. There was self-recognition amongst participants that they were not experts, but rather a "jack of all trades" type of profession. This recognition was a display of personal knowing, in that the participants self-actualised and recognised the limitations of their abilities - a common trait of personal knowing (Clements and Averill, 2004). Furthermore, to be the 'eyes and ears' for other professions, the paramedics must have an adequate perception of a scenario and an intuition of deviations to normality. Paramedics need to interpret situations, engage with them, and respond when needed. These abilities are usually gained from context-specific experiences, and form aesthetic knowing (Cotton and Rodden, 2006).

This 'jack of all trades, master of none' analogy did not undermine the perception of having a duty to protect children and young people. This duty was heavily evident throughout the qualitative findings and could be perceived both in terms of a moral duty and the duty set 
out by the regulating body the HCPC. However, given that the term used was 'duty of care', it seems most likely that this is more a professional/legal reference than an overt moral duty. It can be argued that the same moral obligations underpin those prescribed by legislation and by professional regulations, and as such, participants were nevertheless using and referring an ethical way of knowing. Carper (1978) identifies the ethical pattern of knowing as focusing on matters of obligation or what ought to be done, where moral choices are to be considered in terms of specific actions. This perception of duty is an important one and seems to affirm the need to act on behalf of other professionals.

In acting by proxy for other professionals, the paramedics did note a difference in their roles regarding referring and reporting. The referral was viewed as a support mechanism and the reporting as a punitive one. The word 'report' was used only 56 times throughout all the focus groups, whereas the terms 'refer' or 'referral' were used 212 times. This distinction is made because there appear to be two separately self-identified roles of the paramedic, one to report to protect, and one to refer to support, with some conflation between the two at times. This suggests not only that paramedics perceive themselves as having a role in child protection, but also that they perceive themselves as having different responsibilities within this.

In addition to there being different responsibilities within the paramedics' role in general, subtle differences were identified in the way in which different types of paramedic perceived different elements of practice. Taking, for example, physical abuse, there was a small but statistically significant difference $(.03 \mathrm{sig})$ between the relevance placed upon physical abuse by females (1.22 SD) compared to males (1.34 SD). While there is indeed a recognisable difference, the fact that both sexes at least thought that physical abuse was relevant to their practice is positive. It might be argued that males may have a higher threshold for what they determine to be physical abuse, given masculine traits and the propensity for physical activity and contact sports, which might influence their decision. It must be noted that this is purely speculation, as the author could find no reason linked to the paramedic profession (such as paramedic gender-specific training) which could offer any 
further insights. It is also possible that the female respondents had encountered more physical abuse victims throughout their career, and thus thought it more relevant because of its commonness. This cannot be argued, however, for those staff with 14 or more years of service, as they attributed less relevance to the knowledge of physical abuse than those with 14 years or fewer of service, albeit with a low Cohen's D effect size. It would be reasonable to assert that the longer the employed service, the greater the possibility of witnessing physical abuse, and so the higher the likelihood of finding it more relevant. While it might be possible that staff of 14 or more years had seen little physical abuse in comparison with their years of service, and so thought it less relevant as a result, what is clear is that these two groups at least thought physical abuse was relevant to their practice.

Similarly to physical abuse, there was a statistically significant difference (.01 sig) between the relevance placed upon emotional abuse by females (1.24 SD) than by males (1.39 SD) albeit with a low effect size. It might be argued that females (who still tend to be the main parental figure within the western world) tend to be more emotive than males and thus may have a lower threshold to recognise emotional abuse. Stronger maternal attributes may mean that the threshold for reporting or recognising such abuse is lower given their experience. This may not be true for all females, however, who, for example, may not be mothers. This again is speculative and requires further exploration.

Other statistically significant findings related to relevance include the difference in the number of years as a qualified paramedic, as well as the length of service. For example, there was a statistically significant difference (.01 sig) between the relevance placed upon physical abuse by those who had been qualified between $0-14$ years (1.22 SD) and those who had been qualified for 14 years or more (1.38 SD), which may be explained in several ways. Those who had been qualified for less time were more likely to have been trained and educated more recently and thus were more likely to have received a higher level of education linked to child protection, while those who had been qualified longer may not have received such in-depth education. It might be argued that those who had been qualified longer would have had more experience in clinical practice, and this puts the 
number of physical abuse victims they have seen in context, and so they recorded a more representable measurement of its relevance to their everyday practice.

While the statistical difference between these groups of paramedics and the elements of practice can be measured, what is more difficult to measure is the conflict participants felt when comparing their own personal living, dietary, and disciplinary standards with those of their patients. Paramedics identified with a defined role in child protection, and even identified areas of that role which are considered more relevant than others; however, they were unsure where the threshold point of concern was in some cases. Participants mentioned unclean but happy children and houses being untidy but not dirty as cases where they struggled to decide if they should complete a referral or not. This was a departure from their previously defined role as the 'eyes and ears' for others, as they recognised a difference in personal living, dietary, and disciplinary standards but not a need to refer or report, and thus not a need to be the 'eyes and ears'. These views differed between focus groups and were a representation of how a 'personal' way of knowing was being used to define their role. If they considered it to be far below their standards but not unsafe or a cause for concern, then they had no role to play. However, if standards were far below those of the paramedics and were considered a cause for concern, then the paramedics' role became clear once more. The difficulty with this view is that personal standards of living, diet, and parental discipline, for example, can vary dramatically from person to person. While paramedics identify with a role in child protection, it is unclear to what extent this role is carried out ubiquitously across the profession and what effects this may have.

The analysis of this first research question has shown that paramedics do indeed consider themselves as having a role in child protection. They consider this role to be one of information gatherer, referrer, and reporter of concerns to those whose job it is to investigate further. Paramedics do not perceive this role to be one based solely on superficial elements of clinical practice (such as detection of injuries), but rather as one that also involves socioeconomics and education. Paramedics' comparison of their own personal living, dietary, and disciplinary standards complicates this role, and it is influenced by independent variables, such as gender and number of years qualified. It is nevertheless 
considered an act of duty for paramedics to undertake this complex and evolving task. It is clear that paramedics have identified with a role in child protection, and that the role is defined by their place within the community. This understanding contributes to informing future paramedic practice and policy and contributes heavily to the training which is required to ensure this role is undertaken successfully, which will be explored next.

\section{Research Question 2. How do paramedics perceive the training they receive on child protection?}

The review of the literature has identified that paramedics have a role to play in child protection. The paramedic participants themselves identified with a specific role and defined it to a certain extent. However, training is needed to fulfil this role successfully and safely; the consequences of paramedics not being adequately educated and thus inappropriately managing a child protection concern could have deleterious consequences for children and families. Munro (2011), as part of her review of child protection systems, supports the need for better training for those involved with child protection, with employers, educators, and the profession all taking their full share of responsibility. Specialised training and education has continuously emerged as a key solution to the improvement of child protection services (Reder and Duncan, 2004) and given that even experienced clinicians may fail to recognise and deal with serious incidences of abuse, even in the presence of clear clinical indicators (Bannon and Carter, 2003), it is important to review and critically question the education paramedics receive.

Some paramedics referred to not knowing exactly when to report or refer a child or family, which is an identified training need. Paramedics are required to understand what constitutes abuse, neglect, and other safeguarding concerns, and this uncertainty raises questions about the efficacy of their training and highlights possible discrepancies in standardised practice. All paramedics should be trained to a minimum standard of competency, as set out by the HCPC. Given that all care should be evidence based, there should only be minimal differences in practice across the country, such as in methods of local referral. If some paramedics know when to refer but others do not, this suggests that practice is not standardised and training may be lacking. JRCALC (2017) provides a 
foundation level of standardised information and national guidance to paramedics in their supplementary guidance about various types of abuse and neglect, which is the most substantial inclusion of child protection guidance to date. JRCALC (2017) states that the supplementary guidelines aim to ensure that all staff are aware of, and can recognise, cases of suspected child abuse or children at risk of significant harm. This 2017 guidance was supplementary to the 2016 guidance, and it remains unclear why these more expansive child protection guidelines were not included in that release. Paramedics would have to purchase this supplementary guidance at a personal cost of over $£ 20$, which may limit its purchase and uptake. Moreover, even if paramedics did choose to purchase this supplement, there is still no effective way for ambulance services to determine which paramedics have read the guidance.

In all, $87.3 \%(n=241)$ of the respondents had received child protection training within the last three years. This meets the standard set by RCPCH (2014), who state that staff should receive refresher training every three years as a minimum - with training being tailored to the roles of individuals. However, $12.6 \%(n=35)$ had not received child protection training within the recommended period, which is disconcerting given recent findings from cases of child abuse and neglect. The lack of training could contribute to a low level of confidence and a lack of role identity, and could be seen as a barrier to effective child protection, an issue which will now be explored in more detail. While the questionnaire asked if respondents had received any training, it did not ask how much they had received, or by what methods the training was delivered. Participants in the qualitative research made it clear, however, that the method of delivery of child protection training was as important as those who delivered it.

Participants did not want child protection training to be split up into different elements and to be taught in sections on different days as part of wider more general training updates. Rather, they wanted to see specific full-day child protection courses, with training delivered face-to-face by professionals outside the ambulance service who work directly in child and family /social care, and for it to incorporate scenario-based learning methods. While this is 
what is wanted by paramedics, any changes to current delivery require feasibility studies and educational intervention studies to assess if these methods would be beneficial in improving practice, confidence, and role clarity. The recurrent theme of having outside experts deliver training is a repetition of the 'eyes and ears' reference, in so much as paramedics do not consider themselves to be experts, and thus neither do they consider their learning development paramedics to be complete experts. The participants recognised that the learning development paramedic tutors who normally delivered the training lacked the specific experience that the actual social work team did, not in teaching, but in child protection. Given that learning development paramedics must review, teach, and assess many areas of clinical practice, from CPR to driving, this would appear to be an understandable concern.

Paramedics undertaking these training sessions may also have thought that the learning development officers shared the definition of the role with which they identified, again, perpetuating a proxy approach and a perceived lack of expertise. The wish for external experts to deliver training was not solely out of a want for specific expert education, but also because paramedics wanted to know the entire process from referral to family interaction. They wanted to know if their role played a part in improving children's situations and their request for such expertise and feedback acknowledges that it must be lacking from the current training/practice delivery. Scenario-based learning is prominent in paramedic education and a format they would be used to in other areas of practice. This method of delivery is arguably very well placed for such a practical profession, but expertise is required for it to work. Scenario-based training was seen as an improvement method, so will be explored further later. However, its possible delivery may improve training adequacy. Training needs to include the most up-to-date evidence, as this forms the provision and development of factual and empirical knowing. Carper (1978) characterises empirical knowing in terms of the traditional, positivistic, objectivist, and factual view of a profession. Empirical knowing provides information that is factual, objective, research-based, and, most importantly, verifiable by contemporary standards of outcome measures (Clements and Averill, 2004). Those who would both review these standards and assess measures of 
outcomes appear to be separate from the training paramedic receive; therefore, factual knowing may be inhibited.

Training which is not delivered by experts has questionable relevance for paramedics, who also thought that the style of its delivery could be improved. This information contributes to informing future paramedic education and to understanding the barriers paramedics face to delivering effective child protection. However, these views are in contrast to the results of the quantitative findings, thus raising questions as to how paramedics self-report the adequacy of their training. Although $49.3 \%(n=136)$ strongly agreed and $24.6 \%(n=68)$ of paramedics agreed that their training was adequate for them to practise safely, the qualitative exploration found respondents identifying more with the $23.9 \%(n=66)$ of respondents who neither agreed nor disagreed that their training was adequate. This question was at the beginning of the questionnaire, and perhaps respondents would have answered differently after being presented with the various child protection questions. The focus group participants were surprised that so many quantitative participants felt their training was adequate, but they had had the chance to discuss the different types of abuse and neglect and recognise the limitations in knowledge in a group environment, unlike the questionnaire respondents. Participants commented quite vehemently that there had been little to no training and that far more was needed for them to practise safely. While this suggests that training is occurring, the extent to which the training is sufficient and adequate is questionable. Some were unsure regarding what else could be taught, but voiced a lack of confidence and knowledge in the sexual elements of child protection, such as CSE and FGM, thus evidencing where more training was needed without actually saying it. Indeed, the low levels of reported confidence in some areas of child protection practice indicate a lack of adequate training for paramedics and undermine the quantitative findings. Possible causes for the differences in these findings is that the questionnaire did not make the definition of adequate in context clear enough, or indeed, the paramedics themselves did not comprehend what child protection training was needed to fulfil adequacy until they identified low levels of confidence in certain areas. 
The education paramedics receive can reveal a great deal about the profession and organisation in which they practice, which in turn, can identify where improvements may be needed. To improve and sustain effective child protection systems, Munro (2011) argues, organisations need to move from a compliance culture to a learning one. Organisations and professions should be adaptive, didactic, reflective, and supportive, and a learning culture can be engendered through effective education programmes. Such programmes have been shown to prepare those working with children to confront abusing carers and cope with their own emotional responses with confidence and courage (Bannon and Carter, 2003; Tarr et al., 2013). Paramedics in this research felt their training did not match the importance attributed to this area of practice and felt they needed increased levels of more specific training, which was scenario-based and taught by experts. This information informs future education, which in turn, could inform future practice. It is reasonable to conclude that a lack of training can contribute to a lack of confidence. This was the case within this research, as paramedics referred to the need for more training in the areas where confidence was the lowest, as will be explored next.

\section{Research Question 3: How confident are paramedics in child protection?}

Confidence has long been an important element for those working in child and family health and social care (O'Connor \& Leonard, 2014). The term 'confidence' within the context of health practice has been defined by multiple authors as freedom from doubt in oneself and one's abilities, and an internal feeling of self-assurance and comfort (Capper, 2008). It has been recognised that the development of self-confidence is central to better patient outcomes, effective performance, and the continual learning process (Hayes, 2003). However, there is little research assessing paramedic confidence levels in clinical practice in comparison with other professions, such as nursing. Confidence influences many facets of a practitioners' work, from the decisions they make to the way they feel about making them. Turney et al.'s (2011) review of the research findings related to the child in need assessments between 1999 and 2010 (which included over 100 studies) highlighted how important a factor the confidence of the practitioner was. Turney et al. considered a lack of 
confidence to be a barrier to quality in assessment processes, and high levels of confidence to be a supporting quality in assessment practice. This view is shared by the Department of Health, and the Department for Children, Schools and Families Social Work Task Force (2009), which stated that when practitioners have confidence in their own skills, purpose, identity, and in the system in place to back them up, they have a huge amount to offer. This validates the need to investigate and analyse paramedics' level of confidence in areas of child protection. This element of the research can assist in informing future paramedic practice, policy, and education, and leads on from the role paramedics identify with and the lack of training they reportedly receive.

There appears to be a clear hierarchy of confidence in relation to child protection, with paramedics feeling most confident in their ability to recognise neglect and physical abuse compared to their knowledge of poor diet, housing, social interaction, child development, and all aspects of sexual abuse. This is unsurprising given that neglect and physical abuse are often the most overt types of concern (bruises, bite marks, cigarette burns) when compared to the hidden nature of the sexual elements of child protection practice. Paramedics often undertake a top-to-toe skin-to-skin assessment of patients, which would exclude the genitalia unless there was an overt injury to assess and treat. However, many sexual abuse victims have no physical signs of abuse, and paramedics cannot reply on the obviousness of certain signs to adequately protect children and young people, which may account for their lack of confidence in this area. Similarly, neglect or failure to thrive may also be detected by the fact the child may be underweight, may have pathological fractures, and may have eaten something which they should not have (out of desperation). Moreover, the overall environment in which they are living, the clothes they are wearing, and the time of day of the interaction are all overt signs from which conclusions can be drawn. Thus, confidence should be expected to be higher in these more easily detectable areas. Socioeconomic elements of child protection, however, are taught only in higher education, which some paramedics have not undertaken, and these elements are often more subtle and difficult to assess fully. 
All of these areas were seen as predominantly relevant to practice by respondents, so the contrast between the relevance of these areas and paramedics' lack of confidence indicates a training need. Respondents felt least confident in their ability to recognise sexual abuse, CSE, and FGM (in descending order) to such an extent that this will be explored separately; however, again, this suggests the need for specific training. While the confidence in both neglect and physical abuse were high within the sample, there were still some who were not confident, which is concerning, but understandable. This lack of confidence may be attributed to a lack of training or a lack of adequate training. Furthermore, because of the assumed overtness of these types of abuse, learning development teams may assume that training is not required or not required as often. Nonetheless, without training, even in the most basic and more obvious elements of child protection, confidence cannot be gained, and competence cannot be achieved, and such a lack acts as a barrier to effective child protection.

The extent to which paramedics felt confident in recognising the psychosocial elements of practice was positive given the lack of standardised training or literature identified. However, the lower level of confidence felt by the sample is still disconcerting given the importance of these areas to child and family protection and is indicative of more support and training being required. The lack of confidence in this area may be proportionate to what is considered relevant to paramedic practice. Respondents may have felt less confident because they placed less relevance upon these areas (albeit only slightly), and may have felt comfortable with this. Paramedics are often called to patients for other, more obvious types of concern, such as trauma and general illness, which may detract the focus from other conceptual elements of practice. This may also be seen in emotional abuse. Considered a relatively new concept, emotional abuse can have profound effects on children and young people. It might be argued that overtly emotionally abusive behaviour is unlikely to be witnessed by paramedics, as abusers will adapt their behaviour in front of strangers; however, the symptoms of victimisation may be obvious (withdrawn, anxiety, depression). Only $2.5 \%(n=7)$ of participants were very confident in this area of practice, although $43.8 \%$ ( $n=121$ ) were confident, which is positive. As with neglect and failure to 
thrive, paramedics should focus on the possible symptoms of the effects of abuse and not on witnessing the abuse or neglect itself, as this may aid detection.

The lower level of confidence in these areas may also be due to the short time paramedics spend with their patients, and perhaps the extended amount of time needed to assess fully issues such as social interaction, developmental landmarks, poor diet, and emotional interaction in comparison with neglect and physical abuse. This view, however, needs to be challenged and support given to attempts to change further training. Many professionals also get only a short time with the child and family, such as speech and language therapists, general practitioners, and paediatricians, all of whom may have less than an hour with the child and family at a time, but who may not have the benefit of being within the home environment.

There were statistically significant differences in the confidence felt by paramedics compared to advanced paramedics regarding recognition of neglect $(0.1 \mathrm{sig})$, poor housing (.04 sig), and poor diet (.04 sig), with non-advanced paramedics being more confident than those with advanced skills and education. While this sounds counterintuitive, it might be explained by the emphasis placed upon the education of advanced paramedics at the time of the research. Advanced paramedics were considered advanced by virtue of their ability to more ably assess and treat minor illnesses and injuries, and critical injuries and trauma. This workload arguably reduced their interaction with children and young people and focused their time on those with multiple co-morbidities (such as the elderly) and those at high risk of injury (such as young drivers). This may explain the differences in confidence levels, albeit these were only in three areas of practice and were not ubiquitous across the spectrum of areas presented within the questionnaire. Further to this, the sample sizes were also dramatically different (paramedic $n=241$, advanced paramedic $n=35$ ), and so while the data are interesting, no definitive conclusions can be drawn from this, apart perhaps from additional specific training being needed for all types of paramedics to increase confidence. It must be noted that confidence does not necessarily relate to competence. 
By measuring paramedics' level of confidence and exploring the reasons behind the identified hierarchy of subject confidence, future paramedic practice, education, and policy can be informed. Self-measurement of the confidence, self-knowledge, and selfresponsibility that comes with this knowledge involves personal knowing (Clements and Averill, 2006) and suggests that this type of knowledge must be used in decision-making if respondents recognise their limitations.

There is a clear difference between having knowledge of something and being confident in something. Cotton and Roden (2006) portray this aesthetic knowing as the difference between a person who can play the correct notes on the piano and the virtuosity of the concert pianist. There is not necessarily a correlation between confidence and competence, and so it is possible that while confidence in certain areas is particularly low, paramedics' ability to recognise and act upon concerns is quite high, and vice versa. There is some evidence to suggest that the nursing professions' emphasis on self-assessment to evaluate clinical competence and confidence is less effective than previously thought (Baxter \& Norman, 2011). Levels of self-perceived competence in evidence-based medicine do not correlate well with objectively assessed competence, with participants often being overly confident in their abilities (Lai \& Teng, 2011). With this limitation in mind, however, the levels of measured confidence themselves have been a guide to exploring the reasons thought to be behind them, such as a lack of training, the hidden nature of some types of abuse, and the relevance of certain areas to paramedic practice. Similarly, the low levels of confidence in the sexual elements of child protection may not be indicative of low levels of paramedic competence, but can be indicative of various other issues, which can inform future paramedic practice, policy, and education, so low were these levels of confidence. However, it was considered important to present the analysis of this section separately.

Only $21.0 \%(n=58)$ of respondents indicated that they were confident in identifying sexual abuse, $17.8 \%(n=49)$ in identifying CSE, and only $8.7 \%(n=24)$ in recognising signs of FGM. Participants felt that they would not be confident in something that they did not see often, suggesting that they thought they had not witnessed many confirmed cases of sexual abuse, 
FGM, or CSE. There was confusion amongst participants as to whether they should be looking for signs of sexual abuse often because they assumed that it did not happen often, or only looking for further evidence when presented with suspicions. This perceived underestimation of this type of abuse is disconcerting and should be challenged by additional training and education. Had participants fully understood the estimated frequency of sexual abuse in the UK, their confidence levels may have been even lower. It is thought, for example, that within the UK, 24,012 girls and women are at high risk of or may have already undergone FGM Type III and that 8,913 are at high risk of or may have undergone FGM Type ${ }^{1{ }^{12}}$ (Dorkenoo et al. 2007). In countries and communities where the prevalence of FGM is high, the most common age for the FGM procedure is between six and eight years (Dorkenoo et al., 2007). This is an age where perhaps the comprehension and verbalisation of such abuse is more difficult, and where parental figures largely control the child's movement within communities, in public, and at school. This makes it even more important for paramedics to be confident and competent in their assessment of sexual abuse.

The implications for this are twofold. Not only will the children involved continue to be at risk of FGM, but the paramedics themselves may leave themselves open to legal challenges. The introduction of the Serious Crime Act 2015 made it a legal obligation for HCPs (including paramedics) to report suspicions of or actual FGM, something which, the evidence suggests, they are not confident in recognising. However, given this legal obligation (which many participants were not aware of), paramedics felt less concerned about reporting suspicions and being proved wrong, given that is it their legal obligation to report even small suspicions.

Participants stated that a lack of physical signs made sexual abuse easier to hide and thus more difficult to detect. Furthermore, a lack of training in recognising the nonverbal/physical signs of possible sexual abuse contributed to low levels of confidence. Paramedics felt that sexual abuse was hidden from them because despite any suspicions

\footnotetext{
12 FGM type II is the excision of the clitoris with partial or total removal of the labia minora, and FGM type III is the excision of part or all the external genitalia and stitching/narrowing of the vaginal opening (infibulation).
} 
they might have, they would not examine paediatric genitalia unless indicated to do so by major trauma. This stance is correct, as such examinations should only be carried out by forensically trained paediatricians. The inappropriateness of completing a physical examination on a child in this manner was also prevalent in US-based research into barriers affecting recognition of child abuse. Tiyyagura et al.'s (2016) findings showed that prehospital providers were unable to conduct such examinations, due to a lack of both time and of expertise, and that this hampered effective recognition. This 2016 research was US based and whilst there are inherent differences in education models and scopes of practices between UK and US paramedicine, this research nevertheless suggests that more training is needed to highlight that other more suitable methods of identification are available that make sexual abuse more identifiable than previously thought. While referring to the inability and professional unease of examining children and young people in this way, paramedics did not mention what specific genital signs and symptoms they would be looking for in those abuse victims. This suggests a lack not only of forensic knowledge but also of broader knowledge. Findings of genital injury are rare in sexually abused girls and even rarer in sexually abused boys (Herrmann et al., 2014). Thus, paramedics should not be relying on this type of examination to identify possible sexual abuse. Interestingly, while speaking about this element of practice, the paramedics did not mention the various other physical presentations which may indicate sexual abuse. For example, abdominal pain in children is a symptom paramedics often assess and can be a sign of a urinary tract infection (UTI), and while children can have benign UTIs, repeated UTIs can be due to sexual abuse and can present with abdominal pain, fever, and confusion. Participants omitted to mention these types of signs and symptoms, which are examinable and which may lead them to suspect abuse. This would suggest that the physical signs to which participants referred may be thought only to be obvious genital injury or bleeding. This in itself is indicative of the need for more specific training in the diverse presentations of sexual abuse. Such presentations are not confined to sexual abuse or FGM, but are prevalent in other types of sexual abuse, such as CSE; paramedics lack confidence in this area despite having a key role in identifying such abuse (Kirtley, 2013). 
Despite some limitations in their training and education, the concerns paramedics expressed surrounding this type of abuse and exploitation being hidden are well founded, as seen from the in-depth review of the literature. Often this type of abuse is uncovered only when the victims disclose their abuse, or when young children display an inappropriate level of sexual knowledge for their age. Subtle signs include recent travel to a foreign country without male siblings, or a recent visit to a community 'healer' but not a doctor. A disproportionate fear of strangers (with covering of private parts); large amounts of money, gifts, or toys disproportionate to the family social status; and poor parent and child interaction are all possible causes for concern, which paramedics may be able to detect, but for which training does not appear to have been provided. Paramedics are often not able to be left alone with children as other professionals might be (such as school nurses), which may contribute to the increased feeling of this type of abuse being hidden and confidence remaining low.

Paramedics' confidence also appeared to be low due to the conflict created by the normality of the signs and symptoms of sexual abuse. Children can get UTIs, they can be shy and overly aware of strangers, or conversely, not understand the risk of strangers. Paramedics voiced their difficulty in referring or reporting children and young people based upon a lack of evidence and a sign which may be entirely benign. Some participant views surrounding CSE and FGM specifically appeared to be geographically dependent, and so they lacked confidence if they did not think it occurred in their practice area. This research was undertaken in various locations across the South West of England. Those who did not work in inner-city areas with diverse populations appeared almost to discount the possibility of CSE or FGM happening in areas where the population was considered predominantly white British, and thus the need for more training was discounted. CSE can happen anywhere and is not linked to religion, and while FGM is linked to a small number of religious denominations, it can happen anywhere and is not limited by demography. Sexual abuse is thought to be predominantly interfamilial, with no overt religious or racial indicators, yet it is unclear to what extent all the participants knew this. 
Paramedics portrayed some level of confidence in the 'aesthetic knowing' of when something was not 'right', and described the 'unease' or 'Jimmy Saville' feeling they experienced when they suspected heavily that something was wrong. While their confidence in reporting or confronting this feeling was sometimes lacking, their ability to understand the visceral unease they felt about a situation does display confidence in their comprehension of previous experiences. Some participants stated that they would report concerns based solely on a gut feeling. This displays a level of confidence in that they would do so without any objective data to support them. Those who stated that they would not report their concerns based solely upon gut feeling stated that they would seek out objective evidence to support their feeling, which itself indicates that they had confidence in their original belief. Aesthetic knowing is difficult to quantify, and Carper (1978) typically attributes self-confidence to 'personal knowing'; however, levels of self-confidence can be seen through the understanding of aesthetic knowing and the actions carried out as a result of these feelings. More research is required to investigate what paramedics consider these 'feelings' to be and the experience upon which they base these feelings. Participants may have expressed having low levels of confidence, but this was interpreted by the researcher differently in the case of aesthetic knowing.

It is clear that paramedics' confidence levels in child protection vary depending on the subject in question. While confidence does not necessarily correlate directly with competence, recording to what extent paramedics are confident has allowed for a deeper exploration of the underlying issues affecting practice. These results can be used to inform future paramedic education, policy, and practice. For example, paramedics remained confident in areas which have been historically emphasised in training, but less so in areas which are new to the higher education curriculum. Thus, more training is required across all areas to improve the knowledge surrounding recognition, prevalence, and geography with the aim of increasing confidence and possibly competence. Assertions that sexual abuse is as hidden as the participants perceived it to be also need to be challenged with training and education. Some of the causes for lower levels of confidence could be argued to be a barrier 
to effective child protection, which will be explored next with the aim of informing future paramedic practice, policy, and education.

\section{Research question 4. What barriers exist to paramedics undertaking their role in child protection?}

Some barriers have already been identified through the first three research questions (such as geographical bias, lack of confidence, and lack of training); however, other barriers were also evident throughout. By understanding these barriers and any possible cause, future paramedic practice, policy, and education can be informed. These barriers were identified by asking paramedic respondents if they agreed that there had been times where a child protection referral should have been made but was not, and if so, why not. In all, $54.3 \%$ $(n=150)$ of respondents agreed that with hindsight and experience, they could recall times where referrals should have been completed but were not; while disconcerting, this does at least highlight the extent to which barriers are affecting practice. Some of the responses to this question may be referring to historical practice, when ambulance staff were simply ambulance drivers, and any concerns regarding child protection were dealt with by the police (normally female officers). Only one free-text comment response alluded to this by stating 'before reporting systems were in place'. This indicates one reason why such referrals may not have been made; however, with only one such comment and only $38.4 \%$ $(n=106)$ of respondents having 14 or more years of service, many more recent barriers were identified. It is also reasonable to assert that the increasing amounts of readily available and accessible research evidence, training, and findings from serious case reviews have resulted in the maturing of paramedic awareness of what constitutes a child protection concern. While these factors are likely to have affected some of the responses to this question, many barriers were directly identified. Some of these identified barriers are similar to those found in recent internationally based research. For example, Tiyyagura et al.'s (2016) research titled Barriers and Facilitators to Recognition and Reporting of Child Abuse by Prehospital Providers involved twenty-eight prehospital care providers who participated in a simulated case of infant abuse, before participating in one-on-one semi-structured qualitative debriefs. This research aids in answering the research questions surrounding barriers, but is 
not directly comparable to UK practice. The method of education of paramedics in the US is vastly different to that of the UK-based higher education model. Moreover, this research incorporated the perspectives of providers with varying levels of clinical autonomy and responsibility and not solely what the UK considers to be paramedics. It could be argued that this dilution of expertise limits its applicability to UK-based practice, which again is different due to the lack of an insurance-based system. What this research has identified, though, is a similarity in some barriers to effective child protection on an international scale.

\section{Barrier 1: Only realised in hindsight}

Hindsight is a complex concept and depends very much on the timescales involved and the understanding of what information was available at the time of the decision. Practitioners may have mentioned or selected hindsight in terms of having a better understanding of an event years after it occurred. It is not known, however, what timeframe respondents considered adequate for their view to be considered hindsight, and it would be disconcerting if practitioners did not submit a child protection referral relatively shortly after an event. Munro (2011), in her review of child protection for the DoE, also posits that hindsight can be highly complex and can often distort practitioners' judgement about the predictability of an adverse outcome. Munro explains that once one knows that the outcome was tragic, one looks backwards from it, and it seems clear which assessments or actions were critical in leading to that outcome. This is done in a way that often overestimates how visible the signs of danger were, oversimplifies or trivialises the situation confronting the practitioner, and masks the processes affecting practitioner behaviour before the fact. It is because of this over-simplification that hindsight can itself be a barrier, referred to as 'hindsight bias'. As Munro explains, hindsight bias occurs when people feel that they 'knew it all along', that is, when they believe an event is more predictable after it has become known than it was before it became known. Hindsight bias involves a combination of memory distortion, beliefs about events' objective likelihoods, and subjective beliefs about one's own prediction abilities (Roese and Vohs, 2012). In the context of this research, however, hindsight itself is only a barrier if it stops future learning happening, which in turn, stops referrals being made. 
Hindsight was frequently mentioned by the paramedics and could be an indicator of better child protection education post-event, or possibly the increase in mainstream media coverage of the risks facing children and young people and those missed by various professions. The paramedics alluded to learning within the 'mess room' environment ${ }^{13}$, in which paramedics can talk, reflect, and for want of a better expression, 'gossip' about either their mistakes or the mistakes of someone they know while away from managers or training officers. This peer-assisted learning strategy, which influences both a personal and an aesthetic way of knowing, is established within the literature for undergraduate paramedics (Fox et al., 2015) and is a form of reflective practice. Turner (2015) states that unlike for those practitioners who work directly with their peers and seniors, opportunities for paramedics to discuss at length scenarios and cases with appropriate clinicians can be scarce, and reflection amongst peers can be a productive and supportive process.

Perhaps it is only through this reflective process that paramedics come to realise a referral needed to be made, which again identifies a lack of sufficient education as a barrier to child protection. It would appear from the focus group evidence that this mess room reflection is happening less frequently, as demand rises. Paramedics appear either not to be receiving their breaks, or when they are, they only have time to sit and eat their meal or use the toilet facilities. This increase in demand for ambulance services in recent years and increased inability to have this mess room peer reflection may be a barrier to effective child protection, as experience cannot be shared in a safe environment. This further suggests that more formalised and structured clinical supervision sessions, seen less formally in the mess room, might contribute to learning and thus to improved practice. For this peer reflection to be facilitated, time is needed for crews to be 'stood down' from operational duties, which given the demand for their services, is unlikely to be possible.

\footnotetext{
${ }^{13}$ The term 'mess room' is one used to describe a break room, a quiet room, a lunch area; or any other areas paramedics are given to rest or take their unpaid breaks.
} 
The question must be asked as to what stopped the paramedics recognising child protection concerns at the time in question. Hindsight in this context is identified as a barrier, but it is also acknowledged that other barriers existed, barriers which caused the practitioner not to realise at the time that there was a concern which needed reporting. Furthermore, if the paramedics did not go on to report their concerns once they had realised in hindsight that risks had been identified, then further barriers, such as the fear of getting it wrong or the fear of admitting a mistake, could be identified. The next section discusses some of the identified barriers, some of which might explain why child protection referrals were not made at the time.

\section{Barrier 2: Not enough time or not allowed to return to station to complete referral}

Not having enough time or being allowed to complete a referral was frequently mentioned throughout both phases of this research, and was identified as a barrier to effective child protection at the time of the research. In practical terms, this means that the paramedics believed that a child needed some form of welfare or protection, but they did not or were unable to complete a referral. Participants mentioned the lack of time at the end of the shift, and there being no official overtime or time in lieu policy for the completion of referrals. This was coupled with the expectation of having to return to work the next night or day as rostered, no matter how long the referral form takes. This position is disconcerting and could arguably be in contravention of the HCPC (2016) standards of proficiency, in which paramedics are obligated to ensure that the wellbeing of service users is safeguarded at all times, often through the sharing of information. One might argue perhaps that it is to be expected that when working in an 'emergency' service, there might not be sufficient time to complete necessary paperwork in relation to many aspects of care before needing to attend the next emergency call. This barrier to effective child protection may be further exacerbated by national shortages of staff or of paramedics. Tiyyagura et al.'s (2016) research findings also identified time as a barrier with US pre-hospital providers. The description of time-intensive reporting processes and the fast-paced setting of ambulance work contributed to lack of sufficient time being a barrier to effective reporting. While 
limitations exist within this research, it does identify lack of time as being a barrier on an international scale.

Lack of time is not a concern solely for paramedics, but also appears to affect many professionals, and is addressed in recent guidance. RCPCH (2014), for example, states that all specialist lead professionals must be allowed sufficient time and resources to develop and carry out their role. However, it would appear that those tasked with providing these professionals with information, in the form of referrals, are themselves not being given sufficient time and resources. This reason may not be such a common theme in other areas of child protection where the work environment may be static. Paramedics' work environment, however, changes from hour to hour, as do their job roles, and this may be the first time that 'lack of time' has been highlighted as a barrier to CP to such an extent. RCPCH (2014) state within their guidance that those in roles of protecting children and young people (all paramedics) should be given sufficient time (protected), funding, and resources to fulfil their child protection responsibilities. This view is reiterated by Keeping Children Safe (2012), who posit that to fully risk assess and thus risk manage the safety of children in emergencies, enough time must be given to the referral process, something which does not appear to be happening in the case of paramedics. Perhaps this literature refers to the ongoing process of those who have key child protection-specific roles, but the tenets of such recommendations apply to paramedics with just as much validity, and especially given that paramedics often have access to environments that other professionals do not.

However, even if there is sufficient time to complete the required referral, there may be insufficient facilities in place to complete what is needed, acting as a further barrier, as explored next.

\section{Barrier 3: Poor Facilities}

Poor facilities in which to complete referrals was an identified theme and appears to refer to various methods of child protection referral. Referrals have historically been completed 
on paper and faxed to a child protection lead or social services, and in some areas, this is still the case. This involves having to return to a physical ambulance station, something which paramedics have already identified they do not get the time to do. This fax method has, however, predominantly evolved into the online completion of a prescribed form, which while quicker and more secure, still needs to be completed on a computer at a physical ambulance station. It was also mentioned within the focus groups that staff were using their meal breaks to complete referrals, which is undesirable, and therefore, some staff do not complete referrals because this would take up part of this rest time.

However, changes are being made which mitigate the lack of time and facilities somewhat. Crews can call a phone number and have an office-based administrative staff member complete the dictated referral, though this still relies on the paramedics having extra time at the hospital or on scene to do this ${ }^{14}$, or on control administrative staff not being too busy answering 999 calls. The introduction of ePCRs, however (in the form of an individual laptop or Toughbook on each ambulance), may ease this process and allow paramedics to complete referrals, thus, professionally complying with HCPC (2016) standards, and organisationally complying with the latest guidance from RCPCH (2014). In terms of child protection referrals, the ePCR appeared to have been received well and negated the need for the crew to ask permission to return to a station, especially at a time of high demand. Furthermore, time is also saved, as much of the information needed is already autopopulated into the $\mathrm{CP}$ form, such as address and date of birth. While there are still issues associated with the ePCRs, such as internet signal and battery life, it would appear from focus group evidence that they are contributing to the ease with which referrals can be made. Not all areas at the time of writing had ePCRs, and only some of the UK ambulance services have eCPRs or plan to implement them. Those that have implemented them may now have less of a problem with poor facilities and lack of time acting as a barrier, and this

\footnotetext{
${ }^{14}$ Once at hospital DOH / NHS England expect paramedic ambulances to be there for no longer than 15 minutes. This time includes handing a patient over to nursing staff, moving the patient, cleaning equipment, completion of clinical paperwork, crew toilet breaks, water refreshments, and possible child / adult safeguarding responsibilities; all in 15 minutes. Crews can ask for extra time, but this is monitored and if crews are asking for extra time often, they will be questioned as to why. This may be a reason as to why such a large proportion of participants may have not completed referrals.
} 
may have contributed to how they answered the research question relating to identifying ways of improving paramedic child protection practice. While the ePCR appears to be overcoming some barriers, the paramedics themselves still need to understand when and why a referral is needed, to which further barriers have been identified, such as lack of proof.

\section{Barrier 4: Lack of proof}

Lack of proof was a theme identified as a barrier to effective child protection, which is unsurprising given the lack of clear emphasis upon this in recent child protection guidelines for clinical practitioners. What is clear, however, is that NICE (2013) guidance on when to suspect child maltreatment defines the term 'suspect' as "a serious level of concern about the possibility of child maltreatment but no proof of it". This statement validates paramedics referring or reporting concerns based on suspicion only despite not having any proof, yet this still does not appear to be widely understood. This lack of understanding was also identified in Tiyyagura et al.'s (2016) research, with many pre-hospital providers struggling with the level of proof and degree of suspicion necessary for reporting or referring children and families. It is the responsibility of the paramedic to document possible evidence of maltreatment, such as bruises, unexplained genital injuries, poor living conditions, and concerning relationships. However, all that is needed for a referral to be made are reasonable grounds for suspicion, as there is no onus on practitioners actually to have proof (NICE, 2013). An increased awareness of this may overcome this barrier to effective child protection and better support paramedics to make effective referrals.

A lack of support (to discuss burden of proof or suspicion) may also be a barrier to effective child protection. Paramedics normally work alone in clinical practice, with some services having access to senior clinical support via the radio and or telephone, and so the lack of ability to discuss concerns, alternative explanations, and burdens of likelihood with peers or seniors may affect whether a referral is made. Lone paramedics may be unable to discuss suspicions and thus may not have these condoned, critiqued, or validated, and so they do not refer or report. 
The theme of lack of proof also presented itself within the focus group evidence as a feeling of unfair accusations against parents and caregivers. One free-text comment called it "judging others", something which guidance documents in other areas of practice make clear should not be done. Some participants may consider this lack of proof as being construed as prejudicial and, in some cases, even racist behaviour. This line of thought is derived somewhat from the fact that much if not all of healthcare now is based upon evidence: evidence-based practice. Students and practitioners are taught to support their decisions and assertions with evidence, and to provide evidence to support a specific diagnosis, in much the same way that legal outcomes require a 'beyond reasonable doubt' evidence base. The dichotomy between this line of thought and the line of thought that argues that only a suspicion is needed for paramedics to refer or report can cause confusion and unease, and represents a barrier to effective practice. Exploring the paramedic way of knowing has identified that paramedics most often use aesthetic knowing or gut feeling when deciding to refer or report concerns. Given that this way of knowing is lacking in physical evidence yet is used most often, it might be logical to conclude that the extent to which lack of proof effects referrals may be low. The second way of knowing most often used by paramedics is empirical knowing, that is, factual knowledge. The focus group participants stated that they could not refer or report based on a gut feeling, but this knowledge does allow them to pursue more physical evidence to support their experiential concerns or feelings, thus, providing proof. JRCALC (2017) guidance states that it is not the role of ambulance services to investigate; however, the participants described investigating to support their aesthetic knowing. Despite this guidance, this foundation level of investigation appears necessary to ascertain if there are reasonable grounds for the suspicion of a serious concern, as set out within NICE (2013) guidance.

If, however, the proof is not sought, it might be the case that a referral is not made, which suggests further training is required. It also suggests that paramedics may not feel supported to make a referral and possibly be wrong about it. A lack of feedback, the next issue to be explored, may also perpetuate the view that proof is needed to submit a referral. 
Without feedback, paramedics are left wondering if any progress has been made with the referral and whether a lack of proof has hampered such progress. The concept of 'lack of proof' acting as a barrier to effective child protection has been identified as a contributory factor in child death reviews and requires further work to be undertaken by policymakers, educators, and importantly, trainers, who may be able to provide feedback to referring paramedics.

\section{Barrier 5: Lack of feedback}

JRCALC (2017) states that, where appropriate, all staff involved in cases of suspected abuse should be made aware of the possible outcome and any subsequent action. However, only $47 \%$ ( $n=131$ ) of respondents stated that they regularly received feedback from their referrals, which may in turn also contribute to poor levels of confidence and the perpetuation of paramedics' fear of being wrong acting as a barrier. This lack of feedback appears to be in opposition to the most recent paramedic guidance; however, it is not clear how JRCALC would define 'appropriate'. There may be more complex reasons why paramedics do not receive this suggested feedback. Anecdotally, there is a culture within the ambulance service to report a concern to ensure everything possible has been done for the patient/family, and thus paramedics are not left open to criticism, in what is termed 'covering yourself'. This 'covering yourself' is evident in numerous professional practices; it was certainly evident throughout the focus groups, and appears not to have negative connotations. However, arguably, it might itself contribute to some unnecessary or inappropriate referrals being made. Some referrals may have been better made to primary care services, for example, and not social services. These better-placed referrals may, in turn, increase social services' or the child protection team's workload, and thus may inhibit their ability to provide feedback on every possible case.

To a certain extent, this kind of practice is actually supported in various intercollegiate documents, which speak about having reasonable grounds of suspicion, and there not being such a thing as an inappropriate referral (Sibson and Brain, 2009 B). Given the national shortage of social workers in the UK (Tier 2 Shortage Occupation List Government-approved version 2015), feedback may not be a priority. According to Rizan et al. (2014), feedback 
encapsulates the process of appraising performance with the explicit aim of promoting improvement in subsequent equivalent tasks. It is a basic teaching method within clinical settings. The participants recognised this and stated many times that feedback could help them learn which referrals were appropriate or which were not, and that not receiving feedback was unhelpful in that the paramedics did not know if a referral was appropriate, and if they did not receive feedback, there was an assumption that the referral was indeed not appropriate. Feedback was considered even more important by participants in Tiyyagura et al.'s (2016) study, due to the infrequency of paediatric calls. This is arguably similar to in the UK, as most of the calls to 999 ambulance services are for adult patients, making feedback even more important given the limited opportunities for paramedics to learn in practice. When paramedics encountered similar situations in the future, they did not make referrals because of this assumption, thus meaning that the lack of feedback acted as a barrier. An increase in feedback might ensure that more suitable referrals are made, thus reducing the workload, and so giving receivers more time to provide feedback. Such feedback can be provided in scenario-based example sessions, which again, might assist in a greater number of more suitable referrals being made.

If paramedics were to receive feedback, they might have increased confidence regarding when and when not to refer their concerns or may have a more accurate idea of to whom they should be referring their concerns. This lack of feedback, and the possible lack of appropriate referrals, could be due to a lack of confidence, which was identified in the results as another possible barrier.

\section{Barrier 6: Lack of personal confidence}

The distinction is made at this point between personal confidence and confidence in the referral system, as there is some evidence to suggest that these are two separate issues, and a lack of confidence in the referral system has been discussed in terms of facilities, time, and method of referral. It became evident within the results that paramedics' lack of confidence was inhibiting referrals being made by them. Personal confidence is regarded as an internal feeling, but is largely shaped by external factors, such as skill level, previous 
experience, training, knowledge, and level of support. Where uncertainty and ambiguity ${ }^{15}$ are present, confidence appears to be diminished (Martin et al., 2014). Practitioners lack confidence in their or others' ability to recognises some child protection issues and a lack confidence in their ability to document and justify concerns. This lack of confidence may be a barrier to effective child protection, as it may lead paramedics not to submit referrals whether a need is recognised and not. Confidence has been recognised within the quantitative element of this research and used to determine where training needs are required. It must be noted that confidence does not necessarily correlate positively with competence, and more research is required to determine the causes and effects of poor confidence.

RCGP/NSPCC (2014) argue that written procedures and guidance on child protection will not in themselves protect children, but rather practitioners who are skilled and confident in child protection will. They also argue that employers are responsible for ensuring that their practitioners are confident in carrying out their duties for protecting and promoting children's welfare in addition to the normal responsibilities of the individual practitioner. Given that some respondents either had not recently received training in child protection or were not confident that their training was effective enough for them to practice safely, it is unsurprising that a lack of confidence was acting as a barrier. Furthermore, some participants felt unsupported in that their referrals were not being taken seriously, and that their experience was not validated due to the lack of referral feedback, again making it unsurprising that lack of confidence was a key theme.

These elements of low confidence appeared to result from systemic issues in training, feedback, and education. However, there were more visceral causes of lack of confidence, which included having to discuss referrals with parents and guardians. The theme of 'avoidance of confrontation' became evident, which referred to having to either tell parents of referrals or, in some cases, asking their permission. Given the non-confrontational way in

\footnotetext{
${ }^{15}$ Uncertainty was an identified theme; being mentioned 3 times within questions 18-19.
} 
which referrals can be made (online, ePCR) the confrontation was directly with the parent or guardians. Participants mentioned that this can often be awkward or unpleasant, and at times, can even result in violence; thus, many lacked confidence in undertaking this. Other factors contributing to a lack of confidence included the paramedic's age and level of experience. Given that many newly qualified paramedics are often in their early 20 s, they felt that it was not their place to question and judge the parenting styles of people who are so much their senior. With this also comes a lack of confidence due to the length of time since qualification, and a lack of experience. This lack of confidence requires attention from policymakers, trainers, and educators to ensure that confidence does not prevent suitable referrals from being made, and that didactic supervision sessions can be facilitated to increase confidence possibly. Part of having increased confidence is not solely in submitting a referral, but in submitting a referral which may turn out to be incorrect. This should not be a negative experience, but a didactic one. However, a fear of being wrong was also an identified theme of why referrals were not submitted.

\section{Barrier 7: Fear of being wrong}

The fear of being wrong is particularly prevalent throughout healthcare and not just emergency and unscheduled care (Brady, 2013). Fear of being wrong is not only an established barrier to child protection within general practice (RCGP NSPCC 2014), but has also been noted specifically in paramedic practice. Sibson and Brain (2009 B), for example, note that paramedics may fear wrongly accusing parents; however, they reiterate strongly within the literature that 'there is no such thing as a wrong referral - it is only wrong not to do one' when concerns are noted (Sibson and Brain, 2009 p493). The fear of being wrong is often related to the fear of complaints and fear of punitive and de-registration action. This view is noted within the literature about social workers, as O'Connor and Leonard (2014) suggest that practitioners fears about 'getting it wrong' may feel overwhelming and may affect their capacity to empathise with others. This possible stress amongst many other stressful factors may be a factor in why fears of being wrong stopped the paramedics submitting referrals, despite this element being well advertised as a contributing factor in 
child death reviews. Tiyyagura et al. (2016) identified 'fear of being wrong' as a barrier to effective referral as well, but more so out of fear for the parents and caregivers and the possible repercussions for them. Less fear was voiced over the repercussions for the providers themselves; however, the professional and legislative responsibilities of paramedics differ greatly from the UK to the US.

This theme may be linked to the lack of support paramedics feel, in that they do not always receive feedback to validate or correct their referrals. They perceived that their referrals are not always being taken seriously by agencies, and thus, they could not justify risking being wrong in the face of such possible professional dissonance. However, any such perceived dissonance may be alleviated by sufficient training or education, which may, in turn, contribute to further referrals being made. The fear of being wrong did seem to be overshadowed by the need to 'cover' oneself and submit a referral irrespective of the outcome. A fine balance needs to be struck between submitting appropriate referrals and being comfortable submitting referrals which may indeed be wrong. Without feedback, however, it was difficult to assess fully the extent to which participants identified with 'being wrong'. Arguably, with more knowledge and training, the possibility of submitting incorrect referrals decreases, an issue that is explored in continuation.

\section{Barrier 8: Poor knowledge and training}

Poor knowledge and training were considered a barrier to effective child protection; however, this was somewhat conflated, as $73 \%(n=204)$ of quantitative respondents thought they had received adequate child protection training to ensure that they could practise safely. The questionnaire question itself only asked whether participants felt that they had received adequate child protection training, and was not specific enough to determine differences between certain aspects of child protection, which may account for the fact that poor knowledge and training was later identified both quantitatively and qualitatively as a barrier. In all, $87 \%(n=241)$ of respondents stated that they had received some form of child protection update within the last three years, as set out within the RCGP NSPCC (2014) intercollegiate document. However, the level of training/update was reported as lacking sufficient depth and time. It is entirely possible that until paramedics are made aware of 
what is expected from them and what they are capable of in terms of child protection that they may have unrealistic expectations of training and knowledge, and they may indeed be highly competent. This view is, of course, compared with the $54.3 \%(n=150)$ of respondents who recognised only in hindsight that a referral was actually needed. Without sufficient objective measurement of paramedics' knowledge, such as an exam, the training needs of paramedics cannot be fully assessed. Paramedics rely heavily on their aesthetic knowing when making decisions, which may be a reflection upon any empirical knowing that they have acquired being less adequate.

The RCPCH (2014) recommends that all non-clinical and clinical staff who have any contact with children, young people, and parents/carers (including ambulance staff) have at least 34 hours of refresher training every three years, something which, according to focus group participants, does not happen. Reasons for this lack of training may be high REAP ${ }^{16}$ levels, lack of staff (training and operational), winter pressures, lack of available backfill of staff to cover those in training, or other training needs being prioritised over the need for child protection. While the RCPCH (2014) is a guidance document, this requirement for training is a legislative one. The Children Act 2004, for example, and specifically section 11, places duties upon ambulance services and those who work for them to ensure that they consider the need to safeguard and promote the welfare of children when carrying out their functions (Children Act 2004 Section 11.2a). The Act recognises that health professionals are in a strong position to identify welfare needs or child protection concerns regarding individual children, and, where appropriate, they can provide support (WTSC 2015). To ensure compliance with such legislation, ambulance services must provide training and education to their staff. WTSC (2015) states that employers are responsible for ensuring that their staff are competent to carry out their responsibilities for protecting and promoting the welfare of children, but this does not appear to be happening.

\footnotetext{
16 Resourcing Escalatory Action Plans (REAP) have been adopted by all Ambulance Service Trusts in England to ensure a structured set of arrangements when 'normal' operating functions are challenged, either through loss of staff and resources, or through external factors, including periods of high demand. Each Trust will have a variety of strategic and tactical options in their REAP that are most suitable to deal with the situation (Brotherton 2009).
} 
These barriers were identified through the quantitative and qualitative elements of this research and were provided by paramedics working in practice. Arguably, if the paramedics knew what was acting as a barrier, then they must have known which scenarios required a referral; thus, the training might indeed have been adequate. Despite this being a possibility, it is clear that the paramedics felt the need for more training and that this was seen as a way of improving the efficacy of child protection. This and other improvement strategies are the focus of the next research question.

\section{Research question 5: Can any ways of improving paramedic practice in child protection be identified?}

By answering the first four research questions, the ways in which paramedic practice can be improved have been made evident. While this research has not directly investigated the efficacy of paramedic child protection, it has looked at the barriers involved and at paramedics' feelings. It has identified that in some cases, child protection referrals are not made for various reasons, and that paramedics feel that the lack of feedback and of scenario-based training inhibit learning. Moreover, it has identified that poor technology, poor facilities, poor time management, and a poor understanding of specific subject areas inhibit referrals being made. It is on these experiences and barriers, and on the knowledge that paramedics use in their decision-making, that suggested improvements will be based. These proposed areas will need validation and feasibility studies to ascertain if they contribute towards improvements in paramedic child protection, which itself needs further quantification. While this might be need to be researched further, this section nevertheless contributes to the overall aim of informing future paramedic practice, policy, and education.

\section{Working relationships}

Paramedics do not work in isolation, but rather as part of a collection of professionals who have responsibility to protect children and young people. These professionals include social services, the police, and primary care. Paramedics perceive their role within child protection to be that of being the 'eyes and ears' in the community and working on behalf of these agencies. However, they feel distant from such services and are unsure of the processes undertaken by them. Closer working relationships with such services may increase the 
number of appropriate referrals made to them and increase paramedics' knowledge and confidence. The paramedics in this research wanted to understand the entire process from referral to any family interaction and felt that it was important for them to be involved. Involvement in multi-agency meetings and debriefs appeared to be sporadic across geographical areas, and the participants felt distant from a process that they did not fully understand. Closer bi-directional working relationships and increased communication between various agencies may negate any feelings of abstraction and distance and may contribute to a better understanding of the expectations each professional has of the others. The paramedics felt there was a lack of feedback provided by outside agencies, and this harboured among them feelings of not being taken seriously, which can also be negated if communication improves. Scenario-based learning with social services, for example, is an example of closer working relationships, something the paramedics proposed to improve practice. The participants considered social services to be experts in their field and wanted them to contribute to their own training and confidence, as will be explored next.

\section{Confidence}

Confidence does not always correlate with competence; however, much of the literature has identified that practitioners need to be confident in their ability to practise. Adequate training influences this confidence, as does sufficient feedback and experience in practice. This research has identified that paramedic confidence is currently low in areas such as sexual abuse, CSE, and FGM. To improve confidence in these areas, specific training is needed that is delivered by experts, and that is scenario based. In addition, staff require peer-based clinical supervision whereby they can discuss their errors, concerns, and decision-making in confidence, and fundamentally learn from each other's experience. Regular research is required (during training) to ascertain the key areas of low confidence for paramedics and explore why this might be. One possible reason might be a lack of training, something which can be remedied. 


\section{Training}

Training is integral to achieving proficiency and competency, and in the case of child protection, safety. This research has identified that the paramedics seemed confused about the adequacy of their training, particularly regarding what they should be taught and what more can be taught. There appeared to be ubiquitous views, however, regarding the need for more regular training delivered by experts, such as practice experts from specialist services and not solely by ambulance service training officers, who are perceived as knowledgeable, but who are not always experts. Such training should be delivered every year, in line with recent guidance, and not on overtime but by abstracting staff from duties, as is done in other clinical professions. Investing in such experts and such abstracted training could not only show paramedics that such training is taken seriously, but also could give them more knowledge about the processes of child protection. Moreover, the child protection training needs to be delivered in isolation from other competing training needs and is a recurrent theme in serious case reviews as an area which appears to be overshadowed by other subjects. Training increases competence and if conducted properly, can increase confidence. This, in turn, can lead to more appropriate child protection referrals being made and more children and families being supported.

\section{Technology}

It has been highlighted that a lack of time and insufficient resources contribute to referrals not being made. Since this research was completed, progress has been made in terms of the ePCR, which means that paramedics are better able to search for patient information, connect to national databases, access online guidelines, and importantly, complete referrals while also completing normal paperwork. This has reduced the need for them to have to return to stations and have access to computers. It also represents a faster and more efficient way of sharing information with external agencies and may provide data for research projects of the future. It is important that such ePCRs are implemented across UK ambulance services, and that staff are aware of the improvements they can make to protecting children and young people. What is also important is that paramedics recognise 
when a child protection referral is needed, and for that to improve, researchers need to understand better the decision-making processes of paramedics and what influences them.

\section{Increased understanding of decision-making}

Understanding how paramedics make a decision can also assist in the future planning of training and education. Based upon Carper's 'ways of knowing', it is possible to identify the extent to which paramedics use different knowledge to undertake child protection. Such ways of knowing can then be used to design training packages or to understand better why certain barriers exist. Educational packages may be better tailored for staff who, for example, may have much tacit and aesthetic knowledge from years of experience, but who make less use of up-to-date empirical knowledge, and vice-versa. Knowing both the barriers to referring and how paramedics were thinking at the time they needed to make the decision could help in the logical and evidence-based formation of educational and or policy interventions to ensure safer practice in the future. A summary of these 'knowing' can be found in Figure 7.

\section{Aesthetic knowledge}

Aesthetic knowledge, which is described as a 'gut feeling', or knowledge drawn from 'previous clinical or life experiences', was used 'all of the time' by $19.5 \%(n=54)$ of respondents, with $64.4 \%$ ( $n=178$ ) using such knowledge 'often'. While the use of reflective practice within paramedicine is encouraged, the extent to which practitioners make adequate use of aesthetic knowledge is questionable, given the lack of feedback and training. Thus, some of these previous experiences may have been invalid or incorrect, and so the paramedics' gut feeling may have been wrong. Aesthetic knowledge takes time and experience to accrue, being described as the 'art of a profession'. Those who were unsure if they used aesthetic knowledge or those who stated that they did not use such knowledge often may perhaps have been new to the profession, be relatively early on in their career, be working reduced hours, or possibly not be able to use such knowledge because of a lack of experience in child protection. Educational packages should explore what paramedics consider their gut feelings to be and support this with the 'empirical factual' knowledge 
needed, which was the second most used type of knowledge. Trainers and policymakers should not focus on one type of knowing too greatly because of the variances in the uses. Newer staff may have more empirical knowing, but less aesthetic knowing, and more experienced staff may have less empirical knowing but more aesthetic knowing. This concept was expressed by the focus groups, but not ubiquitously. Understanding what staff themselves consider aesthetic knowing to be is important in designing effective learning packages. Given how aesthetic knowing can differ so much, it is important to have a robust and up-to-date empirical knowledge base. Improving this could improve the way in which paramedics carry out child protection in practice.

\section{Empirical knowledge}

Empirical knowing was used less than aesthetic knowing, but more than personal and ethical. Empirical knowledge represents many different facets of education and practice. In all, $24.6 \%$ ( $n=68)$ stated that they used empirical knowledge 'all the time', and $43.4 \%$ $(n=120)$ stated that they used empirical knowledge (gained from training and education) to inform their decision-making 'often'. This is positive, especially given that $87.3 \%(n=241)$ of the sample had received child protection training within the last three years. This means that the empirical knowledge that paramedics are using is relatively up-to-date, albeit with questionable adequacy. It has already been highlighted that the respondents may have been using empirical knowledge to make decisions from sources of education and training that they considered inadequate; this was heavily evident within the qualitative findings. Therefore, to then practise, or indeed improve the confidence paramedics have in their training, the empirical base of any training needs to be improved, or the training needs to be explained better to paramedics. It is challenging to assert that training needs to improve based purely upon the perception of paramedics. However, given that levels of confidence in areas such as sexual abuse, CSE, and FGM were so low, it can be reasonably argued that the empirical base for this area at least requires improvement. Indeed, this was also evident by the qualitative findings.

Empirical knowledge may not be based solely on education and training courses, however, and can be based on the presentation of objective evidence. This evidence can include 
victim disclosure, witnessing abusive behaviour, or seeing clear signs of physical abuse and neglect within the home. This type of evidence is not always obvious and can be very subtle at times, and sometimes can be actively hidden. Paramedics may critically analyse the objective evidence with which they are presented alongside other more subjective elements to come to a decision. Thus, it is important for paramedics to know what forms objective evidence and what they can do with the evidence. Given the changing nature of child protection and the need to keep abreast with various changes in practice and research findings, the adequacy of the provision of empirical evidence is important; it contributes heavily to paramedics' decision-making, as well as a mixture of legal, contractual, professional, and duty-bound obligations. When presented with this evidence, however, it is important to understand on a personal level what is the right thing to do.

\section{Personal knowledge}

Personal knowing refers to knowing the self; encountering and actualising the concrete, individual self; and developing an interpersonal relationship between the paramedic and the patient (Carper, 1978). Personal knowing involves understanding how this relationship and any potential unconscious bias or opinion may influence decisions, in this case, in relation to child protection; $10.5 \%(n=29)$ of participants said that they used their personal (of themselves) knowledge all the time when making a decision regarding child protection, with a further $51.8 \%(n=143)$ stating that they used it often. There is always a possibility that practitioners are faced with such obvious and unarguable needs to protect children and young people that they would perhaps not need to draw upon their own personal knowledge, but at times, there may be more complex influencing factors. This may account for the $28.6 \%(n=79)$ of respondents who were unsure about using personal knowing, and furthermore, the $7.2 \%(n=20)$ who stated that they did not use it often, and the $1.8 \%(n=5)$ who did not use it at all.

Clements and Averill (2004) argue that words or other descriptors cannot accurately describe personal knowing; rather, it is expressed as a state of being, a personal experience reflecting who practitioners are and who they are becoming. This subjective nature may be again reflected in those respondents who were 'unsure' if they used it or not. Furthermore, 
some paramedics who work remotely (telephone triage) do not very often have this close interpersonal connection with a patient; thus, they may not be able to know within themselves and the patient-practitioner interaction that something may need further investigation or protection. Personal knowing involves reflecting upon oneself in a specific context, and often reflecting with colleagues, to better understand oneself. This research has identified a lack of clinical supervision sessions involving paramedics and a lack of peerbased support networks. Clinical supervision is a recognised and validated activity in healthcare, which brings skilled supervisors and practitioners together to reflect upon their practice. It is a facilitated and structured time for practitioners to think about their knowledge and skills and about how they may be developed to improve care. Workloads and demands upon ambulance services have increased to the extent that even the informal reflective sessions paramedics once had in crew rooms on ambulance stations have now stopped. Without this structured or informal time to reflect with colleagues and alone, it is reasonable to argue that paramedics' personal knowing in relation to child protection is inhibited. Employers and policymakers need to improve the provision of clinical supervision and allow this personal knowing to be developed effectively. Educators need to ensure that personal knowing is not biased towards a specific outcome, but rather, like aesthetic knowing, is a platform from which decisions can be justified through various means. It is important to understand that paramedics come from various backgrounds and that this personal knowing may differ, and so to ensure a consistent and valid approach to effective child protection. Personal knowing should be used in conjunction with guidelines to determine the immediacy or priority of concerns and can be affected by various ethical considerations.

\section{Ethical knowledge}

Ethical knowing can at times be difficult to describe, especially in terms of completing a questionnaire. This way of knowing was used the least by paramedics, which may be reasonable given that a decision may be made based upon empirical knowing at the least. Ethical knowing may be thought to be more prevalent in other areas of paramedicine, such as end-of-life-care, given that if a child is suspected of being neglected or abused, there may 
be no ethical quandary regarding whether to make a referral or not. However, it is often not that simple, and there can be many ethical considerations in child and family health. Ethical knowing is often expressed through ethical codes and standards; it involves identifying and analysing the values and beliefs held by those making the decision, and by those to whom the decision relates. It has been recognised that ethical knowing comprises a complex myriad of moral concepts which some practitioners may consider inappropriate when making decisions regarding protecting children. Some paramedics considered the use of ethical knowing when comparing their standards of living with others; they did not want to judge patients and families and had to consider what was right and wrong. It might be argued that there is a need to remain objective when making decisions surrounding child protection. However, the scenarios themselves are often not solely objective, but involve ethical concepts of need, rights, morality, suffering, and utility. It is perhaps because of this ambiguity and complexity that $26 \%(n=72)$ of respondents were unsure if they used it when making decisions, and that a further $13 \%(n=36)$ stated that they did not use it often. Educators and trainers need to explain the ethical considerations of child protection to paramedics better, especially given that ethicality is referred to within various codes of conduct of registered HCPs. It is difficult to view a referral or report as a harmful act because the act itself does not dictate an outcome; rather, it sets in train an investigation, upon which an outcome is decided. Thus, more research is required to determine if paramedics understand the ethicality of their decision-making. This may be facilitated through feedback sessions, which do not seem to occur and which may be facilitated through clinical supervision sessions, which also seem not to occur. 


\section{Figure 7}

\section{Ways of knowing of paramedics}

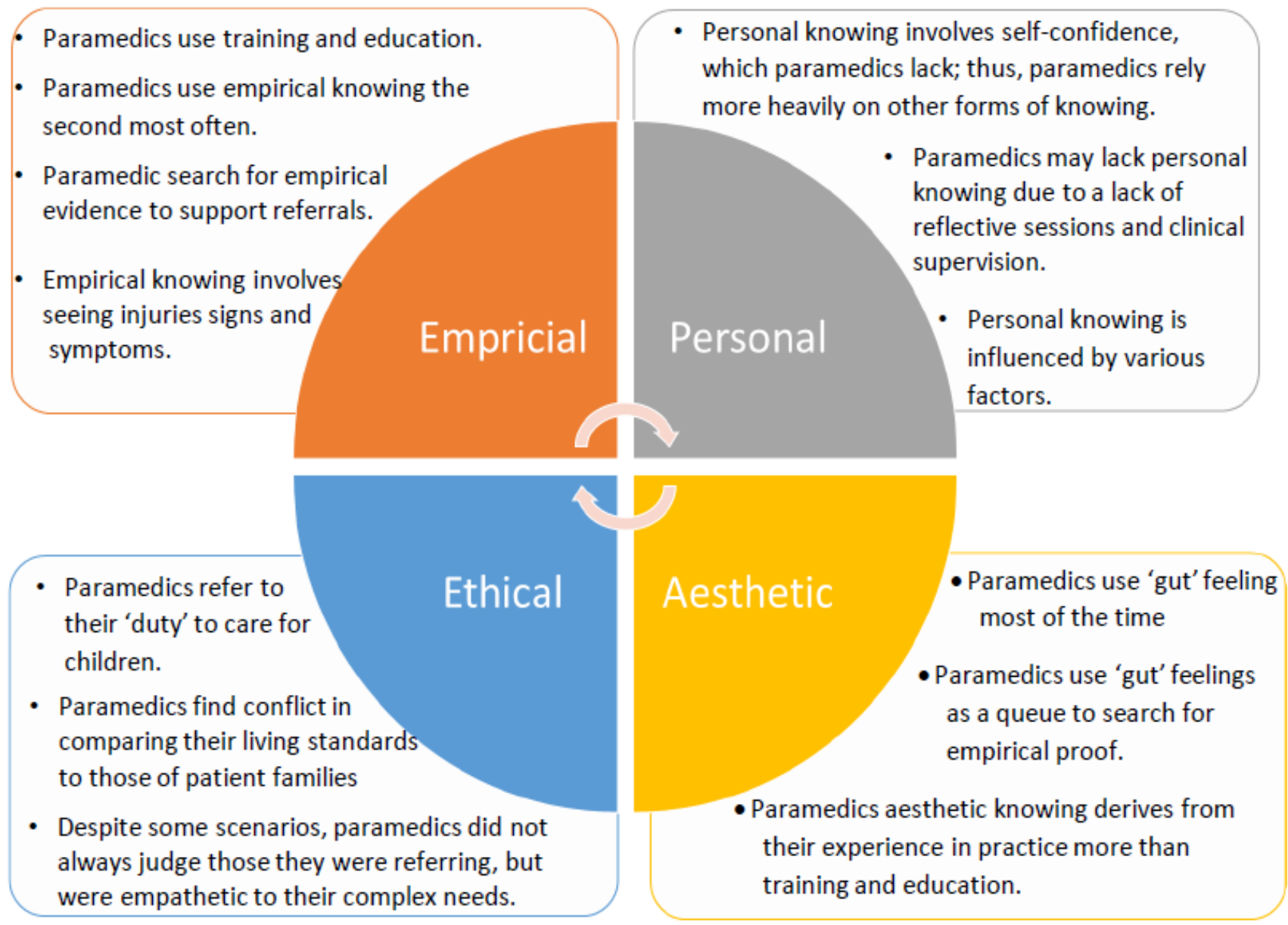

The intention of asking and analysing the possible answers to these research questions has always been to inform future paramedic practice, policy, and education. The in-depth review of the literature identified that paramedics have a key role to play in child protection. It also identified a lack of research into how paramedics themselves perceive this role. By investigating the way paramedics perceive their role in child protection, in a sequential manner, it became evident that this role is shaped by levels of confidence, the adequacy of training, barriers to effective practice, and decision-making processes. It has become evident that improvements are needed in various areas of practice, including the working relationships between paramedics and outside agencies, the amount and method 
of child protection training, the technology used to make referrals, and the understanding of how paramedics come to make decisions. In identifying these areas of improvement, it can be argued that this research can contribute to informing future paramedic practice, policy, and education. While this research can inform future practice, policy, and education, this research journey has been a didactic one in pursuit of a doctorate of philosophy; there are obvious strengths and limitations to many areas of this thesis, and an evaluation of these furthers any possible learning.

\section{Research strengths and limitations}

\section{Research strengths}

The findings of this research are a new and valid addition to the research literature and form a basis upon which more research can be conducted. Despite being undertaken in only one UK ambulance service and to some extent with convenience sampling, there are similarities between the sample ambulance trust and all other trusts in the UK. The history behind UK paramedics and their education is ubiquitous to all publicly funded ambulance services, as are the current pressures, challenges, and technological advances. This research was spread over rural and inner-city areas with an encouraging mix of female and male paramedics from a range of ages and educational backgrounds. Despite a small sample size in comparison with the entire UK sample of paramedics, the findings may still be interpreted by other ambulance trusts, who may also find them valid. There is no way, however, to determine if these findings are generalizable to all other UK ambulance services or if the experiences of these participants are the same as those of other UK paramedics; replication of this study would be needed in other sample ambulance trusts to determine this. However, there is now a point of comparison against which any differences can be analysed, which was not available previously. 


\section{Limitations}

\section{The review of the literature}

Obviously, these findings reflect the practice of one UK ambulance service, which is a limitation for generalizability. The review of the literature was limited by the fact that there remains a relative paucity of data related to paramedics and CP and a lack of literature and research undertaken regarding paramedics. There is an increasing number of textbooks which include $\mathrm{CP}$ either as a caveat to vulnerable patient groups, or as a separate section entirely. There is also an increasing number of academic articles being published related to paramedics and their role and responsibilities in $\mathrm{CP}$; however, these remain largely conceptual, with still little actual research having been undertaken. The newly formed and named 'Prevent' strategy aims to identify and support those children and young people who may be susceptible and at risk of being drawn into terrorism and religious and political extremism. Given that this strategy was only fully formed after the questions were designed, this research did not include it in the thesis; however, 'Prevent' does form a part of paramedic child protection given the contact paramedics have with children and young people in schools and the home environment.

\section{Data collection}

The questionnaire itself was limited in various ways. For example, the questionnaire was too long, with 39 questions, many of which had multiple responses. Many respondents did not complete it in its entirety and indeed may have been put off from even starting it. For an emergency service where respondents may be interrupted at any time by an emergency call, it needed to be shorter and more succinct. This might have increased the possible sample size and reduced the workload of the researcher, who had to analyse a very large data set. Some of the questions within the questionnaire itself were limited in that they were either not specific enough or, as could be seen with hindsight, were not written adequately. Question 38, for example, did not cover every eventuality of the route into the paramedic profession, but those it did cover were written in a verbose and rather complicated way. Furthermore, question 37, which asked what role the participant was currently in, did not cover enough eventualities and conflated certain roles that would have 
been better as separate in terms of data analysis. These were unfortunately not identified at the pilot stage. As mentioned within the methods section, two demographic questions were also limited in that certain measures overlapped and were not separated (0-2 years, 2-4 years), and therefore could not be used and had to be separated into much wider groups of 0-14 years and 14 years + .

The quantitative data collection had inherent limitations by virtue of the use of a Likert scale approach. The Likert approach has been criticised due to its possible limitations. However, given the weakness and lesser reliability of alternative approaches, and that the author negated the Likert limitations by later ratifying the findings with qualitative research, use of such a method, although limited, was deemed justified.

The online delivery methods were limited in their ability to ensure that only paramedics completed the survey. Had non-paramedic staff completed the survey, it would have diluted the ability to answer the research questions effectively. The benefit of the widespread advertisement via trust bulletins, Facebook pages, and so forth offered outweighed the possible limitation of non-paramedic staff choosing to complete the survey, which seemed unlikely.

\section{Sample population}

At the time the questionnaire was released, there were approximately 1,495 paramedics working for the sample ambulance service and approximately 20,199 registered paramedics in the UK; thus, the sample of 276 was only $18.4 \%$ of the ambulance service paramedic workforce and only $1.3 \%$ of the UK paramedic workforce. Similarly, despite the qualitative research having a good mix of respondents with different lengths of service, there was a gender imbalance of more than twice as many females than males, and they were predominantly aged between $35-44$, in a sample of only 25 paramedics. Younger respondents tended to be from a university background where the predominant age group tended to be traditionally trained in-house, which may have caused bias in the qualitative findings. While in qualitative work, more emphasis is placed on what is said and less on how 
many participants were involved to say it, there are obvious benefits of having a larger more representable sample size of paramedics.

\section{Comparison between professions}

It is acknowledged that whilst this research purposefully looked only at paramedics, the fact that only paramedics participated and moreover, only UK paramedics, could be seen as a limitation. Comparative research is a research methodology that aims to make comparisons across different countries or cultures and would normally look to compare practices or health outcomes in different countries and societies (Salway et al., 2011). It is recognised that such models of research have expanded to incorporate institutions, professions, and even families (Paisey \& Paisey, 2010). Comparing findings between different groups can highlight cultural embeddedness and encourage the learning of new lessons about takenfor-granted concepts. It allows an exploration of expectations and differences in experiences, and highlights how differences in concepts, such as age, funding, location, and roles, can affect change. Paramedics from similar educational backgrounds from different countries could have been investigated to provide a level of international and interprofessional comparison, which could lead to the adoption of best practices. It must also be remembered that paramedics do not work in complete isolation, but rather work within the context of a wider community of professionals with whom they interact and to whom they refer their concerns. It would be reasonable to consider it a limitation that the views and perceptions were not gathered from other professions, such as social workers. Through interaction with different professions, such as social workers, the research findings may have been corroborated, and professions could have been given an opportunity to respond to and expand upon findings, such as the lack of feedback. There would therefore seem to be a missed opportunity for mutual learning and joint problem-solving. This is, of course, weighed against the need to complete a doctoral programme with focus and specificity and within the limitations of time, funding, and population access. It would be prudent perhaps to call for follow up research which presents these findings to other professional groups and investigates their views. 
Reflecting upon these limitations as a research student has helped me understand what can be done in the future to ensure more valid and robustly collected data and analysis. Future research might negate some of the sample and generalizability limitations by repeating it nationally across all ambulance services, with fewer but more specific and more accurately written questions. Despite the limitations of this research, it still provides, with confidence and validity, evidence-based suggestions for current and future practice.

\section{Dissemination strategy}

There is little point in undertaking such research and gaining the views of UK paramedics if others are not given the opportunity to determine the relevance of the findings to practice itself. The findings of this research will be disseminated in the following manner:

- dissemination at a local level through presentations to those in charge of training and child protection within the sample trust

- dissemination at a local level through presentations to paramedics who work within the sample trust

- dissemination through the presentation of findings to the national ambulance service safeguarding board

- dissemination at local and national conferences

- publication in emergency medical, paramedical, and social care journals across various levels

\section{Future research}

This research study has answered some questions, but has also generated many others, which require more research. These include:

1) How competent are paramedics in all aspects of child protection as related to their role?

2) Does paramedic confidence in child protection correlate with confidence? 
3) Does more training child protection training improve the competence of paramedics?

4) Does feedback improve the appropriateness and quality of child protection referrals made by UK paramedics?

Such questions, which are unanswered by this research, may benefit the progression of any suggestions resulting from this research.

\section{Conclusion}

As this research has shown, paramedics within the UK are being called upon to provide care to a rapidly expanding population of children and young people from a range of social classes and cultures. Part of this role will involve encountering children and young people who are at risk of neglect and abuse in all its forms. Paramedics have a key role in identifying and protecting these children, and findings from this research give a new understanding of the role they play, the barriers they face, and the reasons why they do not always feel confident to deliver the care they are committed to providing. This section will now briefly conclude how these findings and the new knowledge created may be used to inform paramedic practice, education, and policy as well as research.

In relation to practice, paramedics consider themselves the "eyes and ears" for other expert professionals in the field of child protection, but do not always feel confident in their ability to recognise neglect, or physical and sexual abuse. They also experience barriers to effective child protection because of the high service demand and a lack of feedback in relation to individual cases they have dealt with from other agencies. The findings suggest there is a need for ambulance services to work together to provide regular training and updating sessions for all aspects of child protection. Likewise, in relation to university education, joint sessions between paramedics and social workers on all pre- and post-registration programmes may also lead to a better understanding of the roles each agency plays and encourage the agencies to work more closely with each other in practice. With regard to policy, the findings suggest paramedics, ambulance services, educational providers, and 
regulators need to work together to effect positive change. Child protection policies need to include representation from the field of paramedicine so these may be truly interdisciplinary and reflect the complex nature of child protection in which paramedics plays a crucial role. In conclusion, this study has provided new evidence in relation to the role paramedics play in relation to child protection, and it is hoped that this will be built upon with more practicebased research from a national perspective. By using a mixed methods approach, it has been possible to provide new insights into how paramedics practise and has contributed to the body of knowledge in a hitherto unexplored area of care. In this way, it has demonstrated the importance of ensuring paramedicine continues to be a research-based profession. 


\section{Bibliography}

Acevedo-Garcia, D., Sanchez-Vaznaugh, E., Viruell-Fuentes, E., \& Almeida, J. (2012).

Integrating social epidemiology into immigrant health research: A cross-national framework. Social Science \& Medicine, 75(12), 2060-2068.

http://dx.doi.org/10.1016/i.socscimed.2012.04.040

Agalioti-Sgompou, V., Christie, S., Fiorini, P., Hawkins, V., Hinchliffe, S., \& Lepps, H. et al. (2015). Smoking, drinking and drug use among young people in England in 2014. London: Health and Social Care Information Centre.

Aldridge, J., \& Becker, S. (2003). Children caring for parents with mental illness (1st ed.). Bristol: Policy.

Anderson, J. (2006). Qualitative and quantitative research. California: Grants and evaluations office: Imperial County Office of Education. Retrieved from http://web20kmg.pbworks.com/w/file/fetch/82037432/Qualitative

Archer, F., \& Spencer, C. (2006). Paramedic education and training on cultural diversity: conventions underpinning practice. Journal of Emergency Primary Health Care, 4(3), 1-15.

Ashtekar, S., \& Mankad, D. (2007). Public Health Education in India. Medico - friend - circle bulletin. http://www.mfcindia.org/curissue.pdf.

Association of Ambulance Chief Executives, \& Joint Royal Colleges Ambulance Liaison Committee (JRCALC). (2013). UK ambulance services clinical practice guidelines: 2013. London, United Kingdom: Class Professional Publishing.

Association of Ambulance Service Chief Executives. (2011). Taking Healthcare to the Patient 2: A review of 6 years' progress and recommendations for the future. Association of Ambulance Service Chief Executives.

Association of Ambulance Service Chief Executives. (2015). Annual Report 2015-16: Association of Ambulance Service Chief Executives.

Association of Ambulance Service Chief Executives. (2017). Annual Report 2016-17: Association of Ambulance Service Chief Executives. 
Atieno, O. (2009). An analysis of the strengths and limitation of qualitative and quantitative research paradigms. Problems of Education In The 21st Century, 13.

Azaare, J., \& Gross, J. (2011). The nature of leadership style in nursing management. British Journal of Nursing, 20(11), 672-680. http://dx.doi.org/10.12968/bjon.2011.20.11.672

Bannon, M. (2003). Paediatricians and child protection: the need for effective education and training. Archives of Disease in Childhood, 88(7), 560-562.

http://dx.doi.org/10.1136/adc.88.7.560

Barbour, R. (2007). Doing focus groups (1st ed.). Los Angeles: SAGE Publications.

Barnardo's. (2016). Alcohol, drug and substance misuse. Retrieved 13 September 2016, from http://www.barnardos.org.uk/what we do/our_projects/substance misuse.htm

Barnett, J., Vasileiou, K., Thorpe, S., \& Young, T. (2015). Justifying the adequacy of samples in qualitative interview-based studies. Bath: Faculty of Humanities and Social Sciences, University of Bath.

Barter, C., McCarry, M., Berridge, D., \& Evans, K. (2009). Partner exploitation and violence in teenage intimate relationships. NSPCC. Retrieved from https://www.nspcc.org.uk/globalassets/documents/research-reports/partner-exploitationviolence-teenage-intimate-relationships-report.pdf

Baumbusch, J. (2010). Semi-Structured Interviewing in Practice-Close Research. Journal for Specialists in Pediatric Nursing, 15(3), 255-258. http://dx.doi.org/10.1111/j.1744$\underline{6155.2010 .00243 . x}$

Baxter, P., \& Norman, G. (2011). Self-assessment or self-deception? A lack of association between nursing students' self-assessment and performance. Journal of Advanced Nursing, 67(11), 2406-2413. http://dx.doi.org/10.1111/j.1365-2648.2011.05658.x

Beauchamp, T., \& Childress, J. (2008). Principles of biomedical ethics (6th ed.). Oxford: Oxford Univ. Press.

Becker, J., \& Roe, S. (2005). Drug use among vulnerable groups of young people: findings from the 2003 Crime and Justice Survey. Home Office Research, Development and Statistics Directorate. 
Becker, S., \& Aldridge, J. (2003). Children caring for parents with mental illness: Perspectives of young careers, parents and professionals. Bristol: Policy Press.

Benner, P. (1984). FROM NOVICE TO EXPERT EXCELLENCE AND POWER IN CLINICAL

NURSING PRACTICE. AJN, American Journal Of Nursing, 84(12), 1479. doi:

$10.1097 / 00000446-198412000-00025$

Berelowitz, S., Firmin, C., Edwards, G., \& Gulyurtlu, S. (2012). The Office of the Children's Commissioner's Inquiry into Child Sexual Exploitation in Gangs and Groups. Interim report. London: The Office of the Children's Commissioner's.

Berkman, L. (2009). Social Epidemiology: Social Determinants of Health in the United States: Are We Losing Ground? Annual Review of Public Health, 30(1), 27-41.

http://dx.doi.org/10.1146/annurev.publhealth.031308.100310

Berragan, L. (1998). Nursing practice draws upon several different ways of knowing. Journal Of Clinical Nursing, 7(3), 209-217. http://dx.doi.org/10.1046/j.1365-2702.1998.00146.x

Bilston, B. (2006). A history of child protection. OpenLearn. Retrieved 21 May 2017, from http://www.open.edu/openlearn/body-mind/childhood-youth/working-youngpeople/history-child-protection

Blaber, A. (2012). Foundations for paramedic practice. Berkshire, England: Open University Press.

Blackman, T. (2008). Better Homes, Better Health: The impact of housing on health and social care: overview of the evidence. Durham: Wolfson Research Institute: Durham University, Durham.

Boddy, J. (2013). Research across cultures, within countries: Hidden ethics tensions in research with children and families? Progress in Development Studies, 14(1), 91-103. http://dx.doi.org/10.1177/1464993413490477

Bradford Safeguarding Children Board. (2013). Serious Case Review Executive Summary 2013. Bradford: Bradford Safeguarding Children Board. 
Brady (A), M. (2012). The paramedics' role in safeguarding and protecting children and young people. International Paramedic Practice, 2(3), 90-95.

http://dx.doi.org/10.12968/ippr.2012.2.3.90

Brady (B), M. (2012). Pre-hospital psychosocial care: changing attitudes. Journal of

Paramedic Practice, 4(9), 516-525. http://dx.doi.org/10.12968/ipar.2012.4.9.516

Brady, M. (2013). How to improve patient care by learning from mistakes. Emergency Nurse, 20(9), 32-35. http://dx.doi.org/10.7748/en2013.02.20.9.32.e679

(Brady M (2012) Pre-Hospital psychosocial care: Changing attitudes. Journal of Paramedic Practice 4(9): 516-25). https://doi.org/10.12968/jpar.2012.4.9.516

Braun, V., \& Clarke, V. (2006). Using thematic analysis in psychology. Qualitative Research in Psychology, 3(2), 77-101. http://dx.doi.org/10.1191/1478088706qp063oa

Braun, V., \& Clarke, V. (2013). Successful qualitative research: A practical guide for beginners. (1st ed.). London: SAGE.

British Broadcasting Corporation. (2014). NSPCC refers more child emotional abuse case referrals. BBC UK. Retrieved from http://www.bbc.co.uk/news/uk-27625958

British Red Cross. (2016). Safeguarding Children and Young People Policy. London: British Red Cross. Retrieved from

http://www.redcross.org.uk/ /media/BritishRedCross/Documents/Who\%20we\%20are/Safe guarding\%20Children\%20Young\%20People\%20Policy.pdf

British Sociological Association. (2017). Statement of Ethical Practice. Durham: British Sociological Association.

Byrne, J., \& Humble, A. (2007). An Introduction into Mixed Method Research. Mount Saint Vincent University.

Brooks, F., Magnusson, J., Klemera, E., Chester, K., Spencer, N., \& Smeeton, N. (2015). Health Behaviour in School-aged Children (HBSC): World Health Organization Collaborative Cross National Study. Hertfordshire: University of Hertfordshire, Hatfield.

Bryman, A. (2008). Social research methods (1st ed.). Oxford [u.a.]: Oxford University Press.

Buchanan, D., VanderWeele, T., Kee, R., \& Sadowski, L. (2009). Effect of a housing and case management program on emergency department visits and hospitalizations among 
chronically ill homeless adults. JAMA, 301(17), 1771.

http://dx.doi.org/10.1001/jama.2009.561

Burton, D., Duty, K., \& Leibowitz, G. (2011). Differences between Sexually Victimized and Nonsexually Victimized Male Adolescent Sexual Abusers: Developmental Antecedents and Behavioral Comparisons. Journal of Child Sexual Abuse, 20(1), 77-93.

http://dx.doi.org/10.1080/10538712.2011.541010

Cameron, R. (2011). Mixed Methods Research: The Five Ps Framework. The Electronic Journal of Business Research Methods, 9(2).

Campbell, H., \& Rasmussen, B. (2012). Riding Third: Social Work in Ambulance Work. Health \& Social Work, 37(2), 90-97. http://dx.doi.org/10.1093/hsw/hls012

Campeau, A. (2008). Professionalism Why Paramedics Require 'Theories-of-Practice. Journal Of Emergency Primary Health Care, 6(2), 1-7.

Capper, C. (2008). Confidence. In E. Mason-Whitehead, A. Mclntosh-Scott \& T. Mason, Key Concepts in Nursing (1st ed., pp. 80-81). London: SAGE Publications Ltd.

Caraher, M., \& Coveney, J. (2004). Public health nutrition and food policy. Public Health Nutrition, 7(05). http://dx.doi.org/10.1079/phn2003575

Carers Trust. (2017). About young carers / Carers Trust. Carers.org. Retrieved 31 January 2018, from https://carers.org/about-us/about-young-carers

Carnago, L., \& Mast, M. (2015). Using Ways of Knowing to Guide Emergency Nursing Practice. Journal of Emergency Nursing, 41(5), 387-390.

http://dx.doi.org/10.1016/j.jen.2015.01.011

Carper, B. (1978). Fundamental Patterns of Knowing in Nursing. Advances in Nursing Science, 1(1), 13-24.

Centers for Disease Control and Prevention. (2013). Youth Risk Behavior Surveillance. Atlanta, Georgia: Centers for Disease Control and Prevention.

Center for Surveillance, Epidemiology, and Laboratory Services, Centers for Disease Control and Prevention (CDC), U.S. Department of Health and Human Services, Atlanta. (2013). Youth Risk Behavior Surveillance-United States, 2013. Atlanta: Centers for Disease Control and Prevention. 
Child Welfare Information Gateway. (2016). Definitions of Child Abuse and Neglect. Online: Retrieved 20 May 2017, from https://www.childwelfare.gov/topics/systemwide/lawspolicies/statutes/define/

Children and Young People's Health Outcomes Forum (2012). Report of the children and young people health outcomes forum - Mental health sub-group. Children and Young People's Health Outcomes Forum.

Children's Commissioner. (2013). If only someone had listened: Inquiry into child sexual exploitation in gangs and groups. Children's Commissioner for England.

Children's Commissioner for England. (2014). Protecting children from harm: A critical assessment of child sexual abuse in the family network in England and priorities for action. London: Children's Commissioner for England.

Chinn, P., \& Kramer, M. (2011). Theory and nursing: integrated knowledge development. (8th ed.). St. Louis, Mo.: Mosby.

Clements, P., \& Averill, J. (2006). Finding patterns of knowing in the work of Florence Nightingale. Nursing Outlook, 54(5), 268-274.

http://dx.doi.org/10.1016/i.outlook.2006.06.003

Clements, P., \& Averill, J. (2004). Patterns of knowing as a method of assessment and intervention for children exposed to family-member homicide. Archives of Psychiatric Nursing, 18(4), 143-150. http://dx.doi.org/10.1016/j.apnu.2004.05.006

Cleaver, H., Unell, I. and Aldgate, J. (2011). Children's needs - parenting capacity. Child abuse: parental mental illness, learning disability, substance misuse and domestic violence, 2nd Edition. London: The Stationery Office

Cleveland, H., Herrera, V., \& Stuewig, J. (2003). Journal of Family Violence, 18(6), 325-339. http://dx.doi.org/10.1023/a:1026297515314

Coghlan, D. and Brannick, T. (2005). Doing action research in your own organization, $2^{\text {nd }}$ edn. London, UK: SAGE.

College of Paramedics. (2015). Female Genital Mutilation - Mandatory Duty to Report I News. Collegeofparamedics.co.uk. Retrieved 31 January 2018, from https://www.collegeofparamedics.co.uk/news/female-genital-mutilation-mandatory-dutyto-report 
Collin-Vézina, D., Hébert, M., Manseau, H., Blais, M., \& Fernet, M. (2006). Self-concept and Dating Violence in 220 Adolescent Girls in the Child Protective System. Child and Youth Care Forum, 35(4), 319-326. http://dx.doi.org/10.1007/s10566-006-9019-6

Collishaw, S., Howat, N., Bassett, C., Fisher, H., Bradley, C., \& Corral, S. et al. (2011). Child abuse and neglect in the UK today. The National Society for the Prevention of Cruelty to Children.

Cooklin, A. (2006). Children as carers of parents with mental illness. Psychiatry, 5(1), 32-35. http://dx.doi.org/10.1383/psyt.2006.5.1.32

Continuing Professional Development: Critical thinking for paramedic practice. (2013). Journal Of Paramedic Practice, 5(7), 1-11. doi: 10.12968/jpar.2013.5.7.cpd1

Coughlan, M., Ryan, F., \& Cronin, P. (2008). Undertaking a literature review: A step-by-step approach. British Journal of Nursing, 17(1), 38-43. Retrieved from:

http://www.cin.ufpe.br/ in1002/leituras/2008-undertaking-a-literature-review-a-step-bystep-approach.pdf

Crisp, J., Taylor, C., \& Potter, P. (2001). Potter \& Perry's fundamentals of nursing. Sydney: Mosby.

Croasmun, J., \& Ostrom, L. (2011). Using Likert-Type Scales in the Social Sciences. Journal of Adult Education, 40(1).

Crotty, M. (2012). The foundations of social research (1st ed.). London: SAGE.

Crown Prosecution Service. (2017). Domestic violence: The facts, the issues, the future Speech by the Director of Public Prosecutions, Keir Starmer QC. Cps.gov.uk. Retrieved 18 May 2017, from http://www.cps.gov.uk/news/articles/domestic violence the facts the issues the future/

Darra, S. (2008). Emotion work and the ethics of novice insider research. Journal of Research In Nursing, 13(3), 251-261. http://dx.doi.org/10.1177/1744987107085119

Davey Smith, G., Hart, C., Hole, D., MacKinnon, P., Gillis, C., \& Watt, G. et al. (1998). Education and occupational social class: which is the more important indicator of mortality risk? Journal of Epidemiology \& Community Health, 52(3), 153-160. http://dx.doi.org/10.1136/jech.52.3.153 
Davidson-Arad, B., \& Benbenishty, R. (2010). Contribution of child protection workers' attitudes to their risk assessments and intervention recommendations: a study in Israel. Health \& Social Care in The Community. http://dx.doi.org/10.1111/j.13652524.2009.00868.x

Davidson, E., Pawson, H., Davidson, E., \& Pawson, H. (2006). Fit for purpose? Official measures of homelessness in the era of the activist state. Radical Statistics, 93, 7-29.

Declaration of Helsinki - Ethical Principles for Medical Research Involving Human Subjects. (2008). Wma.net. Retrieved 26 May 2017, from https://www.wma.net/policies-post/wmadeclaration-of-helsinki-ethical-principles-for-medical-research-involving-human-subjects/

Department for Education. (2011). Social work assessment of children in need: what do we know? Messages from research. Bristol: Department for Education.

Department for Education. (2013). Children missing education. Statutory guidance for local authorities and advice for other groups on helping children who are missing education get back into it: Department for Education.

Department for Education. (2016). Child death reviews: year ending 31 March 2016. London: Department for Education.

Department for Education, \& Department for Education. (2013). Children missing education. Department for Education.

Department of Health, and Children, Schools and Families (Jointly). (2009). Building a safe, confident future: The final report of the Social Work Task Force: November 2009. London: (Jointly) Department of Health, and Children, Schools and Families.

Department for Health. (2005). Taking Healthcare to the Patient: Transforming NHS Ambulance Services. London: Department for Health.

Department of Health. (2005). Taking Healthcare to the Patient: Transforming NHS Ambulance Services. London: Department of Health.

Department of Health, England. (2007). A Statistical Study to Estimate the Prevalence of Female Genital Mutilation in England and Wales. London: Department of Health, England. 
Donnelly, E., Oehme, K., \& Melvin, R. (2016). What do EMS personnel think about domestic violence? An exploration of attitudes and experiences after participation in training. Journal of Forensic Medicine (38) 64-9.

Donovan, T. (2014). What the Children and Families Act 2014 means for social work and social workers - Community Care. Community Care. Retrieved 29 December 2017, from http://www.communitycare.co.uk/2014/04/16/children-families-act-2014-means-socialwork-social-workers/

Doody, O., \& Noonan, M. (2013). Preparing and conducting interviews to collect data. Nurse Researcher, 20(5), 28-32. http://dx.doi.org/10.7748/nr2013.05.20.5.28.e327

Doorway. (2010). Definitions of homelessness. Retrieved 13 September 2016, from https://doorwayproject.wordpress.com/about/definition-of-homelessness/

Dorkenoo, E., Morison, L., \& Macfarlane, A. (2007). A Statistical Study to Estimate the Prevalence of Female Genital Mutilation in England and Wales. London: Department of Health, England.

Drake, P. (2010). Grasping at methodological understanding: a cautionary tale from insider research. International Journal of Research \& Method in Education, 33(1), 85-99. http://dx.doi.org/10.1080/17437271003597592

Driscoll, David L.; Appiah-Yeboah, Afua; Salib, Philip; and Rupert, Douglas J., (2007). Merging qualitative and quantitative data in mixed methods research: how to and why not (2007). Ecological and Environmental Anthropology (University of Georgia). 18.

http://digitalcommons.unl.edu/icwdmeea/18

Eagly A. \& Chaiken S. (2007). The advantages of an inclusive definition of attitude. Social Cognition 25 (5), 582-602.

Edlin, A., Williams, B., \& Williams, A. (2010). Pre-hospital provider recognition of intimate partner violence. Journal of Forensic and Legal Medicine, 17(7), 359-362.

http://dx.doi.org/10.1016/i.jflm.2010.08.004

Emmerson, C., Crawford, R., \& Appleby, J. (2009). How cold will it be? Prospects for NHS funding: 2011-17. London: Kings Fund \& Institute for Fiscal Studies. 
Evans, K., Berridge, D., McCarry, M., \& Barter, C. (2009). Partner exploitation and violence in teenage intimate relationships. The National Society for the Prevention of Cruelty to Children.

Evans, A., Snooks, H., Morrison-Rees, S., \& Thomson, C. (2012). Regulation and governance of paramedic-based pre-hospital emergency care: a comparison of international models and assessment of strengths, weaknesses and best practice. Swansea: National Institute for Social Care and Health Research \& Swansea University.

Eysenbach, G., \& Wyatt, J. (2002). Using the Internet for Surveys and Health Research. Journal of Medical Internet Research, 4(2), e13. http://dx.doi.org/10.2196/jmir.4.2.e13

Farrell, S., Fortune, S., Hawton, K., \& Saunders, K. (2012). Attitudes and knowledge of clinical staff regarding people who self-harm: A systematic review. Journal of Affective Disorders, 139(3), 205-216. http://dx.doi.org/10.1016/i.jad.2011.08.024

Farrimond, H. (2013). Doing ethical research (1st ed.). Houndmills, Basingstoke, Hampshire: Palgrave Macmillan.

Farthing, R. (2012). GOING HUNGRY? Young people's experiences of free school meals. London: Child Poverty Action Group and the British Youth Council.

Fawcett, J., Watson, J., Neuman, B., Walker, P., \& Fitzpatrick, J. (2001). On Nursing Theories and Evidence. Journal of Nursing Scholarship, 33(2), 115-119.

http://dx.doi.org/10.1111/j.1547-5069.2001.00115.x

Fernet, M., Blais, M., Manseau, H., Hébert, M., \& Collin-Vézina, D. (2006). Self-concept and dating violence in 220 adolescent girls in the child protective system. Child and Youth Care Forum, 35(4), 319-326. http://dx.doi.org/10.1007/s10566-006-9019-6

Field, A. (2009). Discovering statistics using SPSS (Introducing dtatistical methods) (1st ed.). London: Sage Publications Ltd.

Fitzpatrick, S., Pawson, H., Bramley, G., \& Wilcox, S. (2017). The homelessness monitor: Great Britain 2012.. London: Institute for Housing, Urban and Real Estate Research, HeriotWatt University and Centre for Housing Policy, University of York.

Fishbein M. \& Ajzen J. (1975). Belief, Attitude, Intention and Behavior: An Introduction to Theory and Research. Addison-Wesley, Reading, MA. 
Fox, M., Winship, C., Williams, W., Leaf, S., Boyd, L., McKenna, L., \& Williams, B. (2015). Peer-assisted teaching and learning in paramedic education: a pilot study. International Paramedic Practice, 5(1), 22-28. http://dx.doi.org/10.12968/ippr.2015.5.1.22

Franks, H., Hardiker, N., McGrath, M., \& McQuarrie, C. (2012). Public health interventions and behaviour change: Reviewing the grey literature. 126 (1), 12-17. https://doi.org/10.1016/i.puhe.2011.09.023

Gadda, A., \& Taylor, J. (2015). Child sexual exploitation: where are the nurses? Journal of Advanced Nursing, 72(4), 721-722. http://dx.doi.org/10.1111/jan.12710

Giddings, L. (2006). Mixed-methods research: Positivism dressed in drag?. Journal Of Research In Nursing, 11(3), 195-203. http://dx.doi.org/10.1177/1744987106064635

Gilvarry, E., McArdle, P., \& O'Herlihy, A. (2012). Practice standards for young people with substance misuse problems. London: The Royal College of Psychiatrists Centre for Quality Improvement.

Glencorse, M., Wilson, G., \& Birch, D. (2014). Paramedic perceptions and attitudes to working with patients with alcohol-related injury or illness. Journal of Paramedic Practice, 6(6), 310-318. http://dx.doi.org/10.12968/ipar.2014.6.6.310

Goddard, C. \& Tucci, J. (2008). Responding to child abuse and neglect in Australia. A joint submission to the Australian Government responding to Australia's children: Safe and Well a National framework for protecting Australia's children. Monash University: Australian Childhood Foundation \& Child Abuse Prevention Research Australia.

Good Clinical Practice (GCP). (2009). Nihr.ac.uk. Retrieved 26 May 2017, from https://www.nihr.ac.uk/our-faculty/clinical-research-staff/learning-anddevelopment/national-directory/good-clinical-practice/

Gordon, A. (2002). SurveyMonkey.com-Web-Based Survey and Evaluation System http://www.SurveyMonkey.com. Internet and Higher Education, 5, 83-87.

Gray, D., \& Watt, P. (2013). Giving victims a voice. National Society for the Prevention of Cruelty to Children and Metropolitan police: London.

Gregoire, A., \& Manning, C. (2006). Effects of parental mental illness on children. Psychiatry, 5(1), 10-12. http://dx.doi.org/10.1383/psyt.2006.5.1.10 
Griffiths, P. (2011). Conducting Ethical Research in Paramedic Practice. In P. Griffiths \& G. Mooney, The paramedic's guide to research: an introduction: An Introduction (1st ed.). London: Open University Press.

Grolnick, W. S., Benjet, C., Kurowski, C. O., \& Apostoleris, N. H. (1997). Predictors of parent involvement in children's schooling. Journal of Educational Psychology, 89, 538-548.

Guadron, M. (2008). Identification of patterns of knowing used by rural community health nurses in decision-making (PhD). State University of New York at Binghamton.

Guba, E., \& Lincoln, Y. (1994). Competing Paradigms in Qualitative Research. In N. Denzin \& Y. Lincoln, Handbook of Qualitative Research (1st ed.). California: SAGE Publications.

Guest, G., Namey, E., \& McKenna, K. (2016). How Many Focus Groups Are Enough? Building an Evidence Base for Nonprobability Sample Sizes. Field Methods, 29(1), 3-22. http://dx.doi.org/10.1177/1525822x16639015

Haley, T. (2011). Cultural challenges in getting it right for every child: A reflective account. Journal of Paramedic Practice, 4(3), 161-165. http://dx.doi.org/10.12968/jpar.2012.4.3.161

Halliwell, E., Main, L., \& Richardson, C. (2007). The fundamental facts: The latest facts and figures on mental health. London: Mental Health Foundation.

Hanson, J. (2013). Educational developers as researchers: the contribution of insider research to enhancing understanding of role, identity and practice. Innovations in Education and Teaching International, 50(4), 388-398.

http://dx.doi.org/10.1080/14703297.2013.806220

Hart, C. (1998). Doing a literature review releasing the social science research imagination. SAGE.

Hartley, R. (2012). Paramedic practice and the cultural and religious needs of pre-hospital patients in Victoria. (Doctor of Philosophy (PhD). School of Education Faculty of Arts Education and Human Development Victoria University - Melbourne Australia.

Harwell, M. R., \& Gatti, G. G. (2001). Rescaling ordinal data to interval data in educational research. Review of Educational Research, 71, 105-131. 
Haukoos, J., Bruggman, A., \& Pearson, D. (2007). Out-of-hospital and emergency department utilization by adult homeless patients. Annals of Emergency Medicine, 50(6), 646-652. http://dx.doi.org/10.1016/j.annemergmed.2007.07.015

Hayes, E. (2003). Nurse practitioner self-confidence and attitudes regarding managed care. Journal of The American Academy Of Nurse Practitioners., 15(11), 501-8.

Health and Care Professions Council. (2014). Standards of proficiency - Paramedics. London: Health and Care Professions Council.

Health and Care Professions Council. (2016). Paramedics. Standards of conduct, performance and ethics. Retrieved 12 September 2016, from http://www.hcpcuk.co.uk/aboutregistration/professions/index.asp?id=10

Health and Social Care Information Centre. (2014). Smoking, drinking and drug use among young people in England in 2014. Leeds: Health and Social Care Information Centre.

Health and Social Care Information Centre: Workforce and Facilities Team. (2015). Ambulance Services, England 2014 - 15. London: Health and Social Care Information Centre.

Heath, H. (1998). Reflection and patterns of knowing in nursing. Journal Of Advanced Nursing, 27(5), 1054-1059. doi: 10.1046/j.1365-2648.1998.00593.x

Her Majesty's Stationery Office and Queen's Printer of Acts of Parliament. (2015). Serious Crime Act 2015. (The Stationery Office).

Her Majesty's Stationery Office. (1996). London.

Herring, J. (2012). Medical law and ethics (4th ed.). Oxford: Oxford University Press.

Herrmann, B., Banaschak, S., Csorba, R., Navratil, F., \& Dettmeyer, R. (2014). Physical Examination in Child Sexual Abuse: Approaches and Current Evidence. Deutsches Ärzteblatt International, 111(41). http://dx.doi.org/10.3238/arztebl.2014.0692

Hester, M., \& Pearson, C. (1998). From periphery to centre (1st ed.). Bristol: Policy Press. 
Higher Education Statistics Agency. (2014). HESA Student Paramedic Record 2002/032012/13. Cheltenham: Higher Education Statistics Agency.

Hilal, A., \& Alabri, S. (2013). Using NVIVO for data analysis in qualitative research. International Interdisciplinary Journal of Education, 2(2).

HM Government. (2011). British Crime Survey and police recorded crime. London: HM Government.

HM Government. (2006). Working Together to Safeguard Children 2006: A guide to interagency working to safeguard and promote the welfare of children. London: The Stationery Office.

HM Government: Department for Education. (2010). Working together to safeguard children 2010: A guide to inter-agency working to safeguard and promote the welfare of children. London: The Stationery Office.

HM Government. (2013). Working Together to Safeguard Children 2013: A guide to interagency working to safeguard and promote the welfare of children.. London: The Stationery Office.

HM Government: Department for Education. (2015). Working together to safeguard children 2015: Statutory guidance on inter-agency working to safeguard and promote the welfare of children. London: The Stationery Office.

HM Government: Department for Education. (2018). Working together to safeguard children 2018: Statutory guidance on inter-agency working to safeguard and promote the welfare of children. London: The Stationery Office.

HM Government. (1998). Data Protection Act. London: The Stationery Office.

HM Government. (2001). Health and Social Work Professions Order 2001. London: The Stationery Office.

HM Government. (2005). Mental Capacity Act. London: The Stationery Office.

HM Government. (2003). Every Child Matters. London: The Stationery Office. Retrieved from https://www.gov.uk/government/publications/every-child-matters

HM Government. (2004). Children Act 2004. London: The Stationery Office. 
HM Government. (2012). The Munro Review of Child Protection Progress report: Moving towards a child-centred system. London: The Stationery Office.

HM Government: Department for Children, Schools and Families. (2009). The protection of children in England: a progress report. London: The Stationery Office.

HM Government: Department for Education. (2011). Munro review of child protection: final report - a child-centred system. London: The Stationery Office.

HM Government: Department for Education. (2010). Munro review of child protection: part 1 - a systems analysis. London: The Stationery Office.

HM Government: Department for Health. (2002). The NHS Plan: a plan for investment, a plan for reform. London: The Stationery Office.

HM Government: UK Visas and Immigration. (2015). Tier 2 shortage occupation list. London: HM Government: UK Visas and Immigration.

Hobart, C., \& Frankel, J. (2009). Good practice in safeguarding children (1st ed.).

Cheltenham: Nelson Thornes.

Holloway, I., \& Wheeler, S. (2009). Qualitative research in nursing and healthcare (3rd ed.). Chichester, West Sussex: Wiley-Blackwell.

Holt, S., Buckley, H., \& Whelan, S. (2008). The impact of exposure to domestic violence on children and young people: A review of the literature. Child Abuse \& Neglect, 32(8), 797810. http://dx.doi.org/10.1016/i.chiabu.2008.02.004

Holzer, P., \& Bromfield, L. (2010). Australian legal definitions When is a child in need of protection? Melbourne Australia: Australian Institute of Family Studies.

Home Office. (2013). Domestic violence and abuse. Retrieved 13 September 2016, from https://www.gov.uk/guidance/domestic-violence-and-abuse

Homeless Watch. (2012). Young \& Homeless: A Survey of Services \& Local Authorities. Homeless Watch. London.

Hoonakker, P., \& Carayon, P. (2009). Questionnaire Survey Nonresponse: A Comparison of Postal Mail and Internet Surveys. International Journal of Human-Computer Interaction, 25(5), 348-373. http://dx.doi.org/10.1080/10447310902864951 
Hopewell, S., McDonald, S., Clarke, M., \& Egger, M. (2007). Grey literature in meta-analyses of randomized trials of health care interventions. Cochrane Database of Systematic Reviews. http://dx.doi.org/10.1002/14651858.mr000010.pub3

Horvath, M., Davidson, J., Grove-Hills, J., Gekoski, A., \& Choak, C. (2014). "It's a lonely journey": A Rapid Evidence Assessment on Intrafamilial child sexual abuse. London: Office of the Children's Commissioner.

House of Commons Home Affairs Committee. (2014). Female genital mutilation : The case for a national action plan. London: The Stationery Office. Retrieved from http://www.publications.parliament.uk/pa/cm201415/cmselect/cmhaff/201/201.pdf

Humphrey, C. (2012). Dilemmas in doing insider research in professional education. Qualitative Social Work: Research and Practice, 12(5), 572-586. http://dx.doi.org/10.1177/1473325012446006

Huston, S., \& Hobson, E. (2008). Using focus groups to inform pharmacy research. Research in Social And Administrative Pharmacy, 4(3), 186-205.

http://dx.doi.org/10.1016/j.sapharm.2007.09.001

Irwin, E., Burgess, R., Schwartz, B., \& Mason, R. (2010). Emergency medical services: A resource for victims of domestic violence? Emergency Medicine Journal, 27(7), 561-564. http://dx.doi.org/10.1136/emj.2009.084129

Ivankova, N., Creswell, J., \& Stick, S. (2006). Using Mixed-Methods Sequential Explanatory Design: From Theory to Practice. Field Methods, 18(1), 3-20.

http://dx.doi.org/10.1177/1525822x05282260

Jacobs-Kramer M, Chinn P. (1988) 'Perspectives on knowing: a model of nursing knowledge'. Scholarly inquiry for nursing practice. 1988;2(2):129-144.

Jay, A. (2013). Independent Inquiry into Child Sexual Exploitation in Rotherham 1997 - 2013. Rotherham: Rotherham Metropolitan Borough Council.

Jayasekara, R. (2012). Focus groups in nursing research: Methodological Perspectives. Nursing Outlook, 60(6), 411-416. http://dx.doi.org/10.1016/j.outlook.2012.02.001

Joint Royal Colleges Ambulance Liaison Committee (JRCALC) and Association of Ambulance Chief Executives (Great Britain). (2016). UK ambulance services clinical practice guidelines 2016. Class Publication. 
Joint Royal Colleges Ambulance Liaison Committee. (2017). JRCALC clinical practice supplementary guidelines 2017. [s.I.]: class legal.

Jones, A., \& Pleace, N. (2017). A review of single homelessness in the UK $2000-2010$. London: The Centre for Housing Policy (CHP) at the University of York.

Jones, A., Slater, J., \& Griffiths, P. (2010). The first year experiences of paramedic students in fHigher Education. Swansea: Higher Education Academy.

Journal of Mixed Methods Research / SAGE Publications Ltd. (2017). Uk.sagepub.com. Retrieved 26 May 2017, from https://uk.sagepub.com/en-gb/eur/journal-of-mixedmethods-research/journal201775\#description

Keeping Children Safe. (2012). Safeguarding children in emergencies Toolkit 2. Keeping Children Safe: London.

Kempe, H., Silverman, F., Steele, B., Droegemueller, W., \& Silver, H. (1962). The BatteredChild Syndrome. JAMA: The Journal of the American Medical Association, 181(1), 17. http://dx.doi.org/10.1001/jama.1962.03050270019004

Kessler RC, Berglund P, Demler O, Jin R, Merikangas KR, Walters EE. (2005). Lifetime Prevalence and Age-of-Onset Distributions of DSM-IV Disorders in the National Comorbidity Survey Replication. Archives of General Psychiatry, 62(6) pp. 593-602.

http://dx.doi:10.1001/archpsyc.62.6.593

Kim, K., Lee, A., Eudey, L., \& Dea, M. (2014). Improving Clinical Competence and Confidence of Senior Nursing Students through Clinical Preceptorship. International Journal of Nursing, 1(2). http://dx.doi.org/10.15640/ijn.v1n2a14

Kings Fund. (2006). Securing good care for older people taking a long-term view.. London: Kings Fund.

Kings Fund. (2009). How cold will it be? Prospects for NHS funding: 2011-17. London: Kings Fund.

Kings Fund (2015) “How serious are the pressures in social care?" [ONLINE]. Retrieved from https://www.kingsfund.org.uk/projects/verdict/how-serious-are-pressures-social-care 
Kirtley, P. (2013). If you shine a light you will probably find it: Report of a grass roots survey of health professionals with regard to their experiences in dealing with child sexual exploitation. Derby: NWG Network.

Korbin, j. (1991). Cross-cultural perspectives and research directions for the 21st century. Child Abuse \& Neglect, 15(1), 67-77.

Kroenke, K., Bair, M., Matthias, M., Hollingshead, N., \& Hirsh, A. (2014). The influence of patient sex, provider sex, and sexist attitudes on pain treatment decisions. The Journal of Pain, 15(5), 551-559. http://dx.doi.org/10.1016/j.jpain.2014.02.003

Kushel, M., Perry, S., Bangsberg, D., Clark, R., \& Moss, A. (2002). Emergency department use among the homeless and marginally housed: results from a community-based study. American Journal of Public Health, 92(5), 778-784. http://dx.doi.org/10.2105/ajph.92.5.778

Lai, N., \& Teng, C. (2011). Self-perceived competence correlates poorly with objectively measured competence in Evidence-Based Medicine among medical students. BMC Medical Education, 11(1). http://dx.doi.org/10.1186/1472-6920-11-25

Laming, H. (2003). The Victoria Climbié inquiry. Norwich: TSO.

Laming, H. (2009). The protection of children in England. London: Stationery Office.

Lamont, A., \& Bromfield, L. (2010). History of child protection services. Australian Institute of Family Studies: NCPC.

Latcham, K. (2013). Professional and legal Issues. In V. Nixon, Professional Practice in Paramedic, Emergency and Urgent Care (1st ed.). New Jersey: Wiley-Blackwell.

Lawson, T. (2004). A Conception of Ontology. Cambridge: Faculty of Economics. Sidgwick Avenue. Cambridge.

Lazenbatt, A. (2010). The impact of abuse and neglect on the health and mental health of children and young people. The National Society for the Prevention of Cruelty to Children.

Legal definition of homelessness. (2017). Shelter England. Retrieved 20 May 2017, from http://england.shelter.org.uk/get_advice/homelessness/homelessness an introduction/legal definition of homelessness 
Leibowitz, G., Duty, K., \& Burton, D. (2011). Differences between sexually victimized and nonsexually victimized male adolescent sexual abusers: Developmental antecedents and behavioural comparisons. Journal of Child Sexual Abuse, 20(1), 77-93. http://dx.doi.org/10.1080/10538712.2011.541010

Lenehan, C., \& Children and Young People's Health Outcomes Forum. (2012). Report of the Children and Young People's Health Outcomes Forum.

Letourneau, N., Tramonte, L., \& Willms, J. (2013). Maternal depression, family functioning and children's longitudinal development. Journal of Pediatric Nursing, 28(3), 223-234. http://dx.doi.org/10.1016/j.pedn.2012.07.014

Levy, Y., \& Ellis, T (2006). A systems approach to conduct an effective literature review in support of information systems research. Informing Science Journal, 9, 181-212.

Likert, R. (1931). A technique for the measurement of attitudes (PhD). New York University.

Long, T., \& Chesser-Smyth, P. (2012). Understanding the influences on self-confidence among first-year undergraduate nursing students in Ireland. Journal of Advanced Nursing, 69(1), 145-157. http://dx.doi.org/10.1111/j.1365-2648.2012.06001.x

Luck, M., \& Townsend, R. (2009). Protective jurisdiction, patient autonomy and paramedics: the challenges of applying the NSW Mental Health Act. Journal of Emergency Primary Health Care, $7(4)$.

Lyons, I., \& Paterson, R. (2009). Experiences of older people in emergency care settings. Emergency Nurse, 16(10), 26-31. http://dx.doi.org/10.7748/en2009.03.16.10.26.c6851

Mack, N., Woodsong, C., Macqueen, K., Guest, G., \& Namey, E. (2005). Qualitative Research Methods: A Data Collector's Field Guide (1st ed.). North Carolina: Family Health International.

Madill, A. (2011). Interaction in the semi-structured interview: a comparative analysis of the use of and response to indirect complaints. Qualitative Research in Psychology, 8(4), 333353. http://dx.doi.org/10.1080/14780880903521633

Mahajan, V., Kaur, A., Sharma, A., Azad, C., \& Guglani, V. (2013). Modifiable factors for prevention of childhood mortality. Indian Pediatrics, 51(1), 45-47.

http://dx.doi.org/10.1007/s13312-014-0325-z 
Major, J., \& Walsh, M. (2011). Child sexual abuse: A new approach to professional education. Nursing Standard, 25(37), 35-40.

http://dx.doi.org/10.7748/ns2011.05.25.37.35.c8519

Manning, C., \& Gregoire, A. (2006). Effects of parental mental illness on children. Psychiatry, 5(1), 10-12. http://dx.doi.org/10.1383/psyt.2006.5.1.10

Marshall, G., \& Jonker, L. (2010). An introduction to descriptive statistics: A review and practical guide. Radiography, 16(4), e1-e7. http://dx.doi.org/10.1016/j.radi.2010.01.001

Martin, C., \& Chapman, R. (2014). A mixed method study to determine the attitude of Australian emergency health professionals towards patients who present with deliberate self-poisoning. International Emergency Nursing, 22(2), 98-104.

http://dx.doi.org/10.1016/j.ienj.2013.09.002

Mason, R., Schwartz, B., Burgess, R., \& Irwin, E. (2010). Emergency Medical Services: a resource for victims of domestic violence? Emergency Medicine Journal, 27(7), 561-564. http://dx.doi.org/10.1136/emj.2009.084129

Mat, S., Hassan, H., Abdullah, N., \& Panduragan, S. (2011). Level of confidence among nursing students in the clinical setting. Procedia - Social and Behavioral Sciences, 18, 404407. http://dx.doi.org/10.1016/i.sbspro.2011.05.059

McParland, J., \& Flowers, P. (2011). Nine lessons and recommendations from the conduct of focus group research in chronic pain samples. British Journal of Health Psychology, 17(3), 492-504. http://dx.doi.org/10.1111/j.2044-8287.2011.02050.x

Mental Health Foundation. (2016). Self-harm. Retrieved 20 May 2017, from https://www.mentalhealth.org.uk/a-to-z/s/self-harm

Merton, R. (1972) Insiders and outsiders; a chapter in the sociology of knowledge. American Journal of Sociology, 78(July), 9-47.

Milne, B., Harrington, H., Caspi, A., \& Moffitt, T. (2002). Males on the life-course-persistent and adolescence-limited antisocial pathways: Follow-up at age 26 years. Development And Psychopathology, 14(1), 179-207. 
Ministry of Health and Scottish Home and Health Department. (1966). Report by the Working Party on Ambulance Training and Equipment Part 1- Training. London: Her Majesty's Stationery Office.

Moffitt, T., Caspi, A., Harrington, H., \& Milme, B. (2002). Males on the life-course-persistent and adolescence-limited antisocial pathways: Follow-up at age 26 years. Development and Psychopathology, 14(1), 179-207. http://dx.doi.org/10.1017/s0954579402001104

Moses, J., \& Knutsen, T. (2007). Ways of knowing (1st ed.). Houndmills, Basingstoke, Hampshire: Palgrave Macmillan.

Moss, A., Clark, R., Bangsberg, D., Perry, S., \& Kushel, M. (2002). Emergency department use among the homeless and marginally housed: Results from a community-based study. American Journal of Public Health, 92(5), 778-784. http://dx.doi.org/10.2105/ajph.92.5.778

Muijs, D. (2010). Doing quantitative research in education with SPSS (2nd ed.). California: SAGE Publications.

Munro, E. (2006). Child protection (SAGE Course Companions series) (1st ed.). SAGE Publications Ltd.

Namey, E., Guest, G., Thairu, L., \& Johnson, L. (2008). Data Reduction Techniques for Large Qualitative Data Sets. In G. Guest \& K. MacQueen, Handbook for team-based qualitative research (1st ed.). AltaMira Press.

National Children's Bureau. (2014). Not present, what future? Children missing education in England. London: National Children's Bureau.

National Institute of Clinical Excellence. (2013). When to suspect child maltreatment. Manchester: National Institute of Clinical Excellence.

National Institute for Health Research. (2016). Good clinical practice (GCP). Leeds: National Institute for Health Research.

National Society for the Prevention of Cruelty to Children. (2015). Child protection register statistics UK: 2011 - 2015. London: NSPCC.

National Society for the Prevention of Cruelty to Children (NSPCC). (2016). How safe are our children? London: NSPCC. 
National Society for the Prevention of Cruelty to Children (A). (2012). An introduction to child protection legislation in the UK. Scotland: The National Society for the Prevention of Cruelty to Children.

National Society for the Prevention of Cruelty to Children, Watt, P., \& Gray, D. (2013). Giving Victims a Voice. A joint MPS and NSPCC report into allegations of sexual abuse made against Jimmy Savile under Operation Yewtree. Metropolitan Police Service \& the National Society for the Prevention of Cruelty to Children.

NHS Digital (2017) Hospital Accident and Emergency Activity 2015-16. NHS Digital. https://www.gov.uk/government/statistics/hospital-accident-emergency-activity-2015-to2016. Accessed 14 January 2018.

Nikolova, K., Lwin, K., \& Fluke, J. (2017). Attitudes on the Responsibility for Child Safety: Key Child Protection Worker Characteristics. Journal of Public Child Welfare, 11(3), 318-338. http://dx.doi.org/10.1080/15548732.2016.1278069

Noble, H., \& Smith, J. (2015). Issues of validity and reliability in qualitative research. Evidence Based Nursing, 18(2), 34-35. doi: 10.1136/eb-2015-102054

Noesgaard, C., O'Mara, L., Black, M., Brown, B., Carpio, B., \& Crooks, D. (2008). Development of professional confidence by post-diploma baccalaureate nursing students. Nurse Education In Practice, 5(6), 360-7.

North, C., Moore, H., \& Owens, C. (1997). Go home and rest? (1st ed.). London: Shelter. Nowell, L., Norris, J., White, D., \& Moules, N. (2017). Thematic Analysis. International Journal Of Qualitative Methods, 16(1), 160940691773384. doi: 10.1177/1609406917733847

O’Herlihy, A., McArdle, P., \& Gilvarry, E. (2012). Practice standards for young people with substance misuse problems. The Royal College of Psychiatrists. 
O'Brien, L., \& Parsons, V. (2011). Paramedic clinical decision-making in mental health care: A new theoretical approach. Journal of Paramedic Practice, 3(10), 572-579.

http://dx.doi.org/10.12968/jpar.2011.3.10.572

O'Connor, L., \& Leonard, K. (2014). Decision Making in Children and Families Social Work: The Practitioner's Voice. British Journal of Social Work, 44(7), 1805-1822.

http://dx.doi.org/10.1093/bjsw/bct051

Office for National Statistics. (2015). Compendium: Focus on Violent Crime and Sexual Offences : Year ending March 2015. London: Office for National Statistics.

Office of the Children's Commissioner. (2015). Protecting children from harm: A critical assessment of child sexual abuse in the family network in England and priorities for action. Children's Commissioner for England.

Office of the Children's Commissioner's. (2013). Office of the Children's Commissioner's Inquiry into Child Sexual Exploitation in Gangs and Groups Final Report. London: Office of the Children's Commissioner's.

O'Sullivan, B. (2014). Paramedic attitudes to deliberate self-harming behaviour in Ireland. Journal of Paramedic Practice, 6(3), 116-118. http://dx.doi.org/10.12968/ipar.2014.6.3.116

Owens, C., Moore, H., \& North, C. (1996). Go home and rest? The use of an accident and emergency department by homeless people. London: Shelter.

Page, C., Sbat, M., Vazquez, K. and Deniz Yalcin, Z. (2013), "Analysis of emergency medical systems across the world", in partial fulfilment of the requirements for the degree of Bachelor of Science thesis, Worchester Polytechnic Institute, Worcester.

Paley, J., Cheyne, H., Dalgleish, L., Duncan, E., \& Niven, C. (2007). Nursing's ways of knowing and dual process theories of cognition. Journal of Advanced Nursing, 60(6), 692-701. http://dx.doi.org/10.1111/j.1365-2648.2007.04478.x

Paisey, C., \& Paisey, N. (2010). Comparative research. Journal of Accounting \& Organizational Change, 6(2), 180-199. http://dx.doi.org/10.1108/18325911011048754

Papathanasiou, I., Fradelos, E., Kleisiaris, C., Tsaras, K., Kalota, M., \& Kourkouta, L. (2014). Motivation, leadership, empowerment and confidence: their relation with nurses' burnout. Materia Socio Medica, 26(6), 405. http://dx.doi.org/10.5455/msm.2014.26.405-410 
Parahoo, K. (2006). Nursing research (1st ed.). Basingstoke, Hampshire [England]: Palgrave Macmillan.

Parker, P., McDaniel, H., \& Crumpton-Young, L. (2002). Do Research Participants Give Interval or Ordinal Answers In Response to Likert Scales?. Ergonomics/Human Performance Laboratory, College Of Engineering. Mississippi State University Press. .

Parton, N. (2004). From Maria Colwell to Victoria Climbié: reflections on public inquiries into child abuse a generation apart. Child Abuse Review, 13(2), 80-94.

http://dx.doi.org/10.1002/car.838

Pearson, C., \& Hester, M. (1998). From periphery to centre: Domestic violence in work with abused children. Bristol: Policy Press.

Pearson, D., Bruggman, A., \& Haukoos, J. (2007). Out-of-Hospital and Emergency Department Utilization by Adult Homeless Patients. Annals of Emergency Medicine, 50(6), 646-652. http://dx.doi.org/10.1016/j.annemergmed.2007.07.015

Peate, I. (2014). FGM: The role of front-line staff (Editorial). Journal of Paramedic Practice, 6(5), 221-221. http://dx.doi.org/10.12968/jpar.2014.6.5.221

Petersen, C. 1896. "The yearly immigration of young plaice into the Limfjord from the German Sea." Report of the Danish Biological Station 6:1-48.

Pierce, L., \& Bozalek, V. (2004). Child abuse in South Africa: An examination of how child abuse and neglect are defined. Child Abuse \& Neglect, 28(8), 817-832. http://dx.doi.org/10.1016/i.chiabu.2003.09.022

Pleace, N., \& Jones, A. (2010). A Review of Single Homelessness in the UK 2000-2010. London: Crisis.

Porter, J., Morphet, J., Missen, K., \& Raymond, A. (2013). Preparation for high-acuity clinical placement: confidence levels of final-year nursing students. Advances in Medical Education and Practice, 83. http://dx.doi.org/10.2147/amep.s42157

Porter, S. (2010). Fundamental Patterns of Knowing in Nursing - The Challenge of EvidenceBased Practice. Advances In Nursing Science, 33(1), 3-14. doi:

10.1097/ans.0b013e3181c9d5eb 
Potter P. \& Perry A. (2001) Fundamentals of Nursing. Mosby, Inc, St. Louis, MO, USA.

QSR International. (2017). What is NVivo? QSR international.com. Retrieved 26 May 2017, from http://www.qsrinternational.com/what-is-nvivo

Queen of England, H. (2014). Most Gracious Speech to Both Houses of Parliament. Speech, House of Parliament.

Radford, L., Corral, S., Bradley, C., Fisher, H., Bassett, C., Howat, N., \& Collishaw, S. (2011). Child abuse and neglect in the UK today. London: NSPCC. Retrieved from https://www.nspcc.org.uk/services-and-resources/research-and-resources/pre-2013/childabuse-and-neglect-in-the-uk-today/

Raman, S., Holdgate, A., \& Torrens, R. (2011). Are our frontline clinicians equipped with the ability and confidence to address child abuse and neglect? Child Abuse Review, 21(2), 114130. http://dx.doi.org/10.1002/car.1180

Reder, P. \& Duncan, S. (2004). 'From Colwell to Climbié: inquiring into fatal child abuse'. In The age of inquiry: learning and blaming in health and social care, N. Stanley \& J.

Manthorpe, (Eds.), Routledge, London.

Rees, N., Rapport, F., Thomas, G., John, A., \& Snooks, H. (2014). Perceptions of paramedic and emergency care workers of those who self harm: A systematic review of the quantitative literature. Journal Of Psychosomatic Research, 77(6), 449-456.

http://dx.doi.org/10.1016/i.jpsychores.2014.09.006

Rees, N., Rapport, F., \& Snooks, H. (2015). Perceptions of paramedics and emergency staff about the care they provide to people who self-harm: Constructivist meta-synthesis of the qualitative literature. Journal of Psychosomatic Research, 78(6), 529-535.

http://dx.doi.org/10.1016/j.jpsychores.2015.03.007

Rees, P., \& Rees, C. (2017). Key ingredients of a doctoral thesis. Unpublished.

Reeve, C., Pashen, D., Mumme, H., De La Rue, S., \& Cheffins, T. (2008). Expanding the role of paramedics in northern Queensland: An evaluation of population health training. Australian Journal of Rural Health, 16(6), 370-375. http://dx.doi.org/10.1111/j.1440-

$\underline{1584.2008 .01018 . x}$

Reeve, K. (2011). The hidden truth about homelessness Experiences of single homelessness in England. The Centre for Regional Economic and Social Research \& Crisis. 
Reilly, J., \& Muldoon, O. (2003). Career choice in nursing students: gendered constructs as psychological barriers. Journal of Advanced Nursing, 43(1), 93-100.

Rhodes, K., \& Pollock, D. (2006). The future of emergency medicine public health research. Emergency Medicine Clinics of North America, 24(4), 1053-1073. http://dx.doi.org/10.1016/i.emc.2006.06.003

Richardson, C., Main, L., \& Halliwell, E. (2007). The fundamental facts: The latest facts and figures on mental health. London: Mental Health Foundation.

Richardson, W., Wilson, M., Nishikawa, J., \& Hayward, R. (1995). The well-built clinical question: a key to evidence-based decisions. ACP Journal Club, 123(A12-13).

Ritter, P., Lorig, K., Laurent, D., \& Matthews, K. (2004). Internet versus mailed questionnaires: a randomized comparison. Journal of Medical Internet Research, 6(3), e29. http://dx.doi.org/10.2196/jmir.6.3.e29

Rizan, C., Elsey, C., Lemon, T., Grant, A., \& Monrouxe, L. (2014). Feedback in action within bedside teaching encounters: a video ethnographic study. Medical Education, 48(9), 902920. http://dx.doi.org/10.1111/medu.12498

Roberts, L., \& Henderson, J. (2009). Paramedic perceptions of their role, education, training and working relationships when attending cases of mental illness. Journal of Emergency Primary Health Care (JEPHC), 7(3).

Roese, N., \& Vohs, K. (2012). Hindsight Bias. Perspectives on Psychological Science, 7(5), 411 426. http://dx.doi.org/10.1177/1745691612454303

Royal College of General Practitioners \& National Society for the Prevention of Cruelty to Children. (2014). Safeguarding children and young people. London: Royal College of General Practitioners \& National Society for the Prevention of Cruelty to Children.

Royal College of Paediatrics and Child Health. (2014). Safeguarding children and young people: roles and competencies for healthcare staff. London: Royal College of Paediatrics and Child Health.

Royal College of Psychiatrists. (2012). Self-harm in young people: information for parents, carers and anyone who works with young people. Retrieved 13 September 2016, from http://www.rcpsych.ac.uk/healthadvice/parentsandyouthinfo/parentscarers/self-harm.aspx 
Rubarth, L. (2005). Nursing patterns of knowing in assessment of newborn sepsis. PhD thesis. University of Arizona.

Runyan, D., Cox, C., Dubowitz, H., Newton, R., Upadhyaya, M., \& Kotch, J. et al. (2005). Describing maltreatment: do child protective service reports and research definitions agree? Child Abuse \& Neglect, 29(5), 461-477. http://dx.doi.org/10.1016/i.chiabu.2004.06.015

Ryder, R., Edwards, A., \& Rix, K. (2017). Children missing education. London: National Children's Bureau.

Sadowski, L., Kee, R., VanderWeele, T., \& Buchanan, D. (2009). Effect of a housing and case management program on emergency department visits and hospitalizations among chronically ill homeless adults. JAMA, 301(17), 1771.

http://dx.doi.org/10.1001/jama.2009.561

Saidin, K. (2017). INSIDER RESEARCHERS: CHALLENGES \& OPPORTUNITIES. Proceedings of The ICECRS, 1(1). doi: 10.21070/picecrs.v1i1.563

Saldaña, J. (2012). The coding manual for qualitative researchers (1st ed.). Los Angeles, Calif. [u.a.]: SAGE.

Salkind, N. (2010). Demographics: Encyclopedia of Research design. SAGE Research methods. Retrieved 26 May 2017, from http://methods.sagepub.com/reference/encyc-of-researchdesign/n108.xml

Salkind, N. (2014). 100 questions (and answers) about statistics (3rd ed.). SAGE. London.

Salway, S., Higginbottom, G., Reime, B., Bharj, K., Chowbey, P., \& Foster, C. et al. (2011). Contributions and challenges of cross-national comparative research in migration, ethnicity and health: insights from a preliminary study of maternal health in Germany, Canada and the UK. BMC Public Health, 11(1). http://dx.doi.org/10.1186/1471-2458-11-514

Schleicher, D., Smith, T., Casper, W., Watt, J., \& Greguras, G. (2015). It's all in the attitude: The role of job attitude strength in job attitude-outcome relationships. Journal Of Applied Psychology, 100(4), 1259-1274. http://dx.doi.org/10.1037/a0038664

Schnell, M., \& Heinritz, C. (2006). Forschungsethik (1st ed.). Bern: Huber, Hans.

Schneller, K. (2012). Intermediate care for homeless people: Results of a pilot project. Emergency Nurse, 20(6), 20-24. http://dx.doi.org/10.7748/en2012.10.20.6.20.c9347 
Schuh, E., \& Barab, S. (2008). Chapter 7: Philosophical perspectives. In J. Spector, M. Merrill, J. van Merrienboer \& M. Driscoll, Handbook of research on educational communications and technology (3rd ed.). Abingdon: Routledge.

Schultz P.R. and Meleis A.I. (1988) Nursing Epistemology: Traditions, Insights, Questions. Journal of Nursing Scholarship 20(4) 217- 221.

Shuler, P., \& Davis, J. (1993). The Shuler Nurse Practitioner Practice Model: A Theoretical Framework for Nurse Practitioner Clinicians, Educators, and Researchers, Part 1. Journal Of The American Academy Of Nurse Practitioners, 5(1), 11-18. doi: 10.1111/j.17457599.1993.tb00835.x

Scotland, J. (2012). Exploring the philosophical underpinnings of research: relating ontology and epistemology to the methodology and methods of the scientific, interpretive, and critical research paradigms. English Language Teaching, 5(9). http://dx.doi.org/10.5539/elt.v5n9p9

Scottish Office: Central Research Unit. (2009). Poor Housing and ill health: A summary of research evidence. Edinburgh: Scottish Office: Central Research Unit.

Self-Harm. (2017). Rcpsych.ac.uk. Retrieved 20 May 2017, from http://www.rcpsych.ac.uk/healthadvice/problemsdisorders/self-harm.aspx

Seto, M., Babchishin, K., Pullman, L., \& McPhail, I. (2015). The puzzle of intrafamilial child sexual abuse: A meta-analysis comparing intrafamilial and extrafamilial offenders with child victims. Clinical Psychology Review. 39:42-57. doi: 10.1016/j.cpr.2015.04.001.

Shaban, R. (2005). Accounting for assessments of mental illness in paramedic practice: A new theoretical framework. Journal of Emergency Primary Health Care, 3(3).

Shelman, E., \& Lazoritz, S. (2005). The Mary Ellen Wilson child abuse case and the beginning of children's rights in 19th century America (1st ed.). Jefferson, N.C.: McFarland \& Company.

Shelter. (2016). What is homelessness? Retrieved 13 September 2016, from http://england.shelter.org.uk/get advice/homelessness/homelessness an introduction/what is homelessness 
Shenton, A. (2004). Strategies for ensuring trustworthiness in qualitative research projects.

Education For Information, 22(2), 63-75. doi: 10.3233/efi-2004-22201

Shi, L., Tsai, J., \& Kao, S. (2009). Public health, social determinants of health, and public policy. Journal Medical Science, 29(2).

Sibson, L., \& Brain, L. (2009 A). Safeguarding children: Role of health professionals. Journal of Paramedic Practice, 1(12), 493-500.

Sibson, L., \& Brain, L. (2009 B). Safeguarding children part 2: reporting and acting on abuse. Journal of Paramedic Practice, 1(13), 539-544.

http://dx.doi.org/10.12968/jpar.2009.1.13.44892

Sibson, L. (2011). A Duty of Care. Journal of Paramedic Practice, 3(11), 601-601. http://dx.doi.org/10.12968/jpar.2011.3.11.601

Simmel, G. (1950). The sociology of George Simmel. New York: Free Press.

Slep, A., Heyman, R., \& Snarr, J. (2011). Child emotional aggression and abuse: Definitions and prevalence. Child Abuse \& Neglect. http://dx.doi.org/10.1016/j.chiabu.2011.07.002

Smart, G. (2009). The role of the paramedic in health promotion. Journal of Paramedic Practice, 1(14), 593-597. http://dx.doi.org/10.12968/jpar.2009.1.14.45380

Smeaton, E. (2009). Children and Young People on the Streets in the UK. Railway Children. https://www.railwaychildren.org.uk/media/11700/off-the-radar-full-report.pdf

Smeeton, N., Spencer, N., Chester, K., Klemera, E., Magnusson, J., \& Brooks, F. (2015). HBSC England National Report 2014. Hatfield, UK: University of Hertfordshire. Retrieved from: http://researchprofiles.herts.ac.uk/portal/en/publications/hbsc-england-national-reporthealth-behaviour-in-schoolaged-children-hbsc(8308a2b1-2599-4bc0-92df5cdf8483c9d8)/export.html

Smith, K., Flatley, J., Chaplin, R., \& Home Office Statistical Bulletin. (2011). Crime in England and Wales 200 9/10: Findings from the British Crime Survey and police recorded crime $\left(3^{\text {rd }}\right.$ edn). London: Home Office. Retrieved from https://www.gov.uk/government/uploads/system/uploads/attachment data/file/116417/h osb1011.pdf 
Smyth, A., \& Holian, R. (2008). Credibility Issues in Research from within Organisations. In P. Sikes \& A. Potts (Eds.), Researching education from the inside (pp. 33-47). New York, NY: Taylor \& Francis.

Soini, H., Kronqvist, E., \& Huber, G. (2011). Epistemologies for qualitative research: qualitative psychology nexus: Vol. 8 (1st ed.). Tübingen, Germany.: Center for Qualitative Psychology.

South Western Ambulance Service (SWAST). (2018). Safeguarding policy and procedures $f$ or children, young people and adults at risk. Exeter: South Western Ambulance Service.

St John Ambulance. (2016). Safeguarding policy. London: St John Ambulance. Retrieved from https://www.sja.org.uk/sja/pdf/Safeguarding-policy2.pdf

Stoltenborgh, M., van IJzendoorn, M., Euser, E., \& Bakermans-Kranenburg, M. (2011). A Global Perspective on Child Sexual Abuse: Meta-Analysis of Prevalence around the World. Child Maltreatment, 16(2), 79-101. http://dx.doi.org/10.1177/1077559511403920

Stuart, G., Wolfe, D., Tortolero, S., Shorey, R., \& Temple, J. (2013). Importance of gender and attitudes about violence in the relationship between exposure to interparental violence and the perpetration of teen dating violence. Child Abuse \& Neglect, 37(5), 343-352. http://dx.doi.org/10.1016/i.chiabu.2013.02.001

Stuckey, H. (2013). An overview of the rationale for qualitative research methods in social health. Journal of Social Health and Diabetes, 1(1), 6. http://dx.doi.org/10.4103/2321$\underline{0656.109828}$

Stuewig, J., Herrera, V., \& Cleveland, H. (2003). Abusive males and abused females in adolescent relationships: risk factor similarity and dissimilarity and the role of relationship seriousness. Journal of Family Violence, 18(6), 325-339.

http://dx.doi.org/10.1023/a:1026297515314

Talwar, D. (2016). Thousands of children 'missing' from school. BBC News. Retrieved 31 January 2018, from http://www.bbc.co.uk/news/education-38145058

Tarr, J., Whittle, M., Wilson, J., \& Hall, L. (2013). Safeguarding children and child protection education for uk trainee teachers in higher education. Child Abuse Review, 22(2), 108-115. http://dx.doi.org/10.1002/car.2275 
Team, I., Violence, Organization, W., \& World Health Organization. (1999). Report of the consultation on child abuse prevention. Geneva: World Health Organization. Retrieved from: http://apps.who.int/iris/handle/10665/65900

Temple, J., Shorey, R., Tortolero, S., Wolfe, D., \& Stuart, G. (2013). Importance of gender and attitudes about violence in the relationship between exposure to interparental violence and the perpetration of teen dating violence. Child Abuse \& Neglect, 37(5), 343-352. http://dx.doi.org/10.1016/i.chiabu.2013.02.001

Thabane, L., Thomas, T., Ye, C., \& Paul, J. (2008). Posing the research question: not so simple. Canadian Journal of Anesthesia/Journal Canadien D 'anesthésie, 56(1), 71-79. http://dx.doi.org/10.1007/s12630-008-9007-4

The College of Podiatry. (2006). An introductory guide to putting research into practice 2. London: Podiatry Now.

The Crown Prosecution Service. (2016). The CPS: Domestic violence. Retrieved 13 September 2016, from http://www.cps.gov.uk/publications/prosecution/domestic/

The Nuremberg Code (1947). (1947).

The United Nations International Children's Emergency Fund. (2014). FACT SHEET: A summary of the rights under the Convention on the Rights of the Child. The United Nations International Children's Emergency Fund.

Thomas, J. (2011). Analysing Thurstone and Likert attitude scales as data collection methods. Journal of Paramedic Practice, 3(5), 250-254.

http://dx.doi.org/10.12968/jpar.2011.3.5.250

Tiyyagura, G., Gawel, M., Alphonso, A., Koziel, J., Bilodeau, K., \& Bechtel, K. (2016). Barriers and facilitators to recognition and reporting of child abuse by prehospital providers.

Prehospital Emergency Care, 21(1), 46-53.

http://dx.doi.org/10.1080/10903127.2016.1204038

Todres, J. (2012). Maturity. Houston Law Review, 48, 1107-1165. Retrieved from https://ssrn.com/abstract=2008754

Townsend, R., \& Luck, M. (2009). Protective jurisdiction, patient autonomy and paramedics: the challenges of applying the NSW Mental Health Act. Journal of Emergency Primary Health Care (JEPHC), 7(4). 
Trickett, P., Mennen, F., Kim, K., \& Sang, J. (2009). Emotional abuse in a sample of multiply maltreated, urban young adolescents: Issues of definition and identification. Child Abuse \& Neglect, 33(1), 27-35. http://dx.doi.org/10.1016/j.chiabu.2008.12.003

Turner, H. (2015). Reflective practice for paramedics: a new approach. Journal of Paramedic Practice, 7(3), 138-144. http://dx.doi.org/10.12968/ipar.2015.7.3.138

Turney, D., Platt, D., Selwyn, J., \& Farmer, E. (2011). Social work assessment of children in need. London: HM Government.

United Nations Children's Fund (UNICEF). (2013). UN Convention on the Rights of the Child (UNCRC) - Unicef UK. Unicef UK. Retrieved 31 January 2018, from

https://www.unicef.org.uk/what-we-do/un-convention-child-rights/

United Nations Children's Fund (UNICEF). (2014). Childhood defined. Unicef.org. Retrieved 31 January 2018, from https://www.unicef.org/sowc05/english/childhooddefined.html

Vagi, K., O’Malley Olsen, E., Basile, K., \& Vivolo-Kantor, A. (2015). Teen dating violence (physical and sexual) among us high school students. JAMA Pediatrics, 169(5), 474.

http://dx.doi.org/10.1001/jamapediatrics.2014.3577

Venkatesh, V., Brown, S., \& Bala, H. (2013). Bridging the qualitative-quantitative divide: guidelines for conducting mixed methods research in information systems. MIS Quarterly, $37(1)$.

Vydelingum, V., \& Colliety, P. (2011). The need for a culturally sensitive approach to care. In R. Davies \& A. Davies, Children and young people's nursing: principles for practice (1st ed., pp. 57-91). London: Hodder Education.

Walker, L., \& Clendon, J. (2012). 'Being young': A qualitative study of younger nurses' experiences in the workplace. International Nursing Review, 59(4), 555-61. Retrieved from http://www.ncbi.nlm.nih.gov/pubmed/23134141

Wanless, D. (2006). Securing good care for older people: Taking a long-term view. London: King's Fund.

Waples, C., Weyhrauch, W., Connell, A., \& Culbertson, S. (2010). Questionable defeats and discounted victories for Likert rating scales. Industrial and Organizational Psychology, 3(04), 477-480. http://dx.doi.org/10.1111/j.1754-9434.2010.01274.x 
Warm, D., Raj, T., Millward, L., \& Canning, U. (2004). Drug use prevention among young people: a review of reviews. NHS Health Development Agency.

Welsh Assembly Government. (2009). Research Governance Framework for Health and Social Care in Wales. Cardiff: Welsh Assembly Government.

Whelan, S., Buckley, H., \& Holt, S. (2008). The impact of exposure to domestic violence on children and young people: A review of the literature. Child Abuse \& Neglect, 32(8), 797810. http://dx.doi.org/10.1016/j.chiabu.2008.02.004

White, J. (1995). Patterns of knowing: Review, critique, and update. Advance Nursing Science, 13, 73-86.

Whitnell, J. (2012). Safeguarding Children. In A. Blaber, Foundations for paramedic practice: A theoretical perspective (2nd ed., pp. 153-174). Open University Press.

Williams, A., Williams, B., \& Edlin, A. (2010). Pre-hospital provider recognition of intimate partner violence. Journal of Forensic and Legal Medicine, 17(7), 359-362.

http://dx.doi.org/10.1016/j.jflm.2010.08.004

Williams, J. (2012). Qualitative research in paramedic practice: an overview. In P. Griffiths \& G. Mooney, The paramedic's guide to research: An introduction (1st ed., pp. 73-74). Berkshire: Open University Press.

Willms, J., Tramonte, L., \& Letourneau, N. (2013). Maternal depression, family functioning and children's longitudinal development. Journal of Pediatric Nursing, 28(3), 223-234. http://dx.doi.org/10.1016/j.pedn.2012.07.014

World Health Organization (2017) 10 facts on mental health. Who.int. Retrieved 25 May 2017, from http://www.who.int/features/factfiles/mental health/mental health facts/en/

World Health Organization. (2011). Suicide data. Retrieved 19 May 2013, from http://www.who.int/mental_health/prevention/suicide/suicideprevent/en/

World Health Organization. (2016). Female genital mutilation. Retrieved 13 September 2016, from http://www.who.int/mediacentre/factsheets/fs241/en/ 
World Health Organization. (2011). 10 facts on mental health. Retrieved from

http://www.who.int/features/factfiles/mental_health/mental_health_facts/en/index7.html

World Health Organization. (2003). Investing in Mental Health. World Health Organization.

World Health Organization. (2017). Child maltreatment. Retrieved 21 May 2017, from http://www.who.int/mediacentre/factsheets/fs150/en/

World Health Organization. (1948). Definition of health. Retrieved 25 May 2017, from http://www.who.int/suggestions/faq/en/

World Health Organisation: Department of Mental Health and Substance Dependence, Noncommunicable Diseases and Mental Health. (2003). Investing in mental health. Geneva. Retrieved from http://www.who.int/mental_health/media/investing mnh.pdf

World Health Organization. (2016) Classification of female genital mutilation. Retrieved 20 May 2017, from http://www.who.int/reproductivehealth/topics/fgm/overview/en/

Wu, H., \& Leung, S. (2017). Can Likert Scales be Treated as Interval Scales? -A Simulation Study. Journal Of Social Service Research, 43(4), 527-532. doi:

$10.1080 / 01488376.2017 .1329775$

Zakrison, T., Hamel, P., \& Hwang, S. (2004). Homeless People's Trust and Interactions with Police and Paramedics. Journal of Urban Health: Bulletin of the New York Academy of Medicine, 81(4), 596-605. http://dx.doi.org/10.1093/jurban/ith143 


\section{Appendices}

\section{Appendix 1: Landmark UK Cases}

\begin{tabular}{|c|c|}
\hline Case & Description \\
\hline $\begin{array}{l}\text { Victoria } \\
\text { Climbié }\end{array}$ & $\begin{array}{l}\text { Victoria Climbié died at the hands of her mother after years of physical abuse. Her post } \\
\text { mortem showed history of } 128 \text { separate injuries because of being beaten with a range of } \\
\text { sharp and blunt instruments. } \\
\text { Victoria's case was formally closed by Haringey social services on } 25 \text { February } 2000 \text {, the day } \\
\text { on which her hypothermic and unconscious body in multi system failure was taken into an } \\
\text { A\&E department, shortly before her death (Laming 2003). } \\
\text { The Climbié Inquiry (Lamming report) details how despite being seen by numerous } \\
\text { professionals over a course of months, none had identified her abuse. }\end{array}$ \\
\hline $\begin{array}{l}\text { Peter } \\
\text { Connelly }\end{array}$ & $\begin{array}{l}\text { Baby Peter Connelly, known also as 'Baby } \mathrm{P}^{\prime} \text { and 'Child A', was a 17-month-old child who died } \\
\text { at the hands of his mother, her boyfriend, and mutual acquaintances on } 3 \text { August } 2007 . \\
\text { Initial examination showed bruising to his body, a tooth missing, a torn frenum, and marks to } \\
\text { his head. Further to this, a tooth was found in his colon, an eight fractured ribs and a } \\
\text { fractured spine were detected; the cause of death was described as a fracture dislocation of } \\
\text { the thoraco-lumbar spine (Serious Case Review (SCR): Child A Haringey council 2009). } \\
\text { There were over ten public agencies involved in the care of Baby P at the time of his death, } \\
\text { which, a later SCR highlighted, all lacked urgency, thoroughness, and sufficient focus of child } \\
\text { welfare (SCR: Child A Haringey council 2009). }\end{array}$ \\
\hline $\begin{array}{l}\text { Daniel } \\
\text { Pelka }\end{array}$ & $\begin{array}{l}\text { Daniel Pelka was a 4-year-old boy murdered at the hands of his parents on } 3 \text { March } 2012 . \\
\text { Pathological examinations revealed that he had suffered over a prolonged period, involving } \\
\text { starvation, assault, neglect, and abuse. } \\
\text { The SCR review highlighted that too many opportunities were missed, included practitioners' } \\
\text { inability to link events, notice patterns, and see through the manipulative, deceitful and } \\
\text { evasive presentation of Daniels parents, a task made more difficult by Daniels siblings not } \\
\text { being abused themselves. }\end{array}$ \\
\hline
\end{tabular}




\begin{tabular}{|l|l|}
\hline $\begin{array}{l}\text { Operation } \\
\text { Yewtree }\end{array}$ & $\begin{array}{l}\text { Jimmy Savile was an English dis jockey, charitable fundraiser, and children's television } \\
\text { presenter posthumously alleged to have abused over } 300 \text { victims, spanning over three } \\
\text { decades. }\end{array}$ \\
$\begin{array}{l}\text { Operation Yewtree and its subsequent report Giving Victims a Voice highlighted how Savile } \\
\text { committed most his abuse during a time where both social attitudes and the workings of the } \\
\text { criminal justice system were completely different to those of today. } \\
\text { The watershed case empowered many victims of non-recent sexual abuse to come forward } \\
\text { to the authorities, whom they believed would now take them seriously. }\end{array}$ \\
\hline
\end{tabular}


Appendix 2: Salient points - focus groups

FF

Last updated $2 / 03 / 16$

Focus group questions

(Paramedics were asked about how relevant certain areas of practice were their role in child protection, which showed mixed results) What do you think a paramedic's role in child protection is?

no history - snap Shot -

- Ever fess - Stat of potman-

Do to u turn t framed as. hod erst ( $73 \%$ agreed there was adequate $\mathrm{CP}$ training) What child protection training do you think should be provided for paramedics in practice?

Adequate - So man Vorabies -

- Carse awdenes -

core scorers.

Foe - bo -bee-

(Sexual $22 \%$ - CSE 19\% - FGM - 10\%) Why do you think paramedics may lack confidence in recognising signs sexual abuse, child sexual exploitation, and female genital mutilation?

15

80

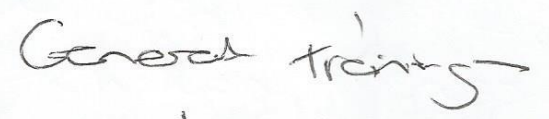

- What fere de' wick child-

- Forms Moor 'bo complete -

(Paramedics gave different reasons for what would make them complete a referral) What would make you decide to complete a child protection referral?

$c$

Didn't feel Right"

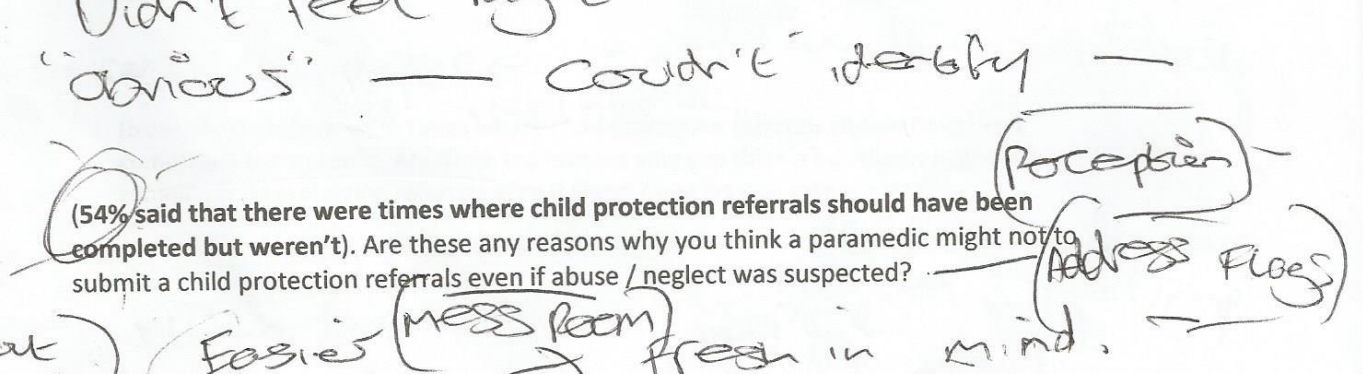

Wis tat

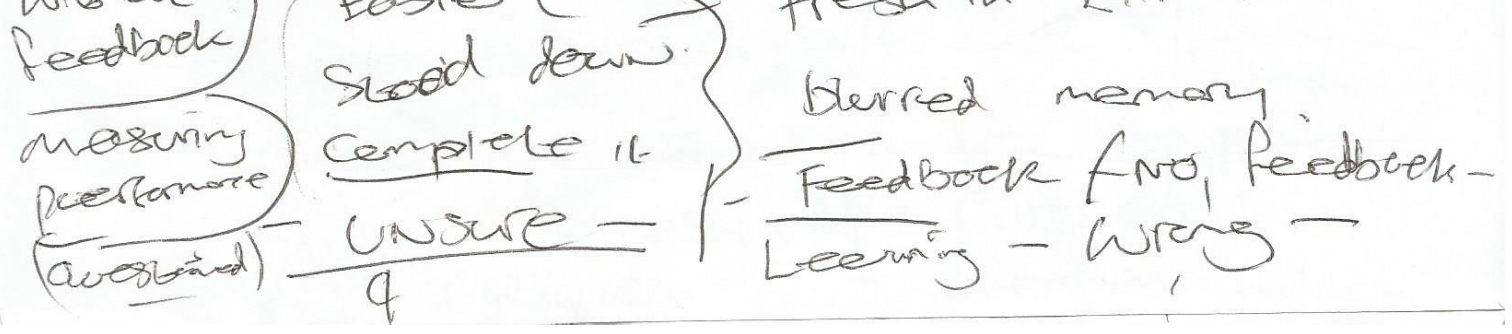

283 
Appendix 3: Thematic coding

"Obvious"

What would make you decide to complete a child protection referral?

Parental concern/Guordien/"Mum" was sting"

"Triggered" "Deep feeling of urease".
Dint feel Right/Comfertable/Feeling/Gut/instinct "'never normowh wrong" - What is it- 'experience". "inisteke" Seem quite right. "Finger on it". "l Don 't know".

"Jimmy sowile feeding" "Bock of mind" "Voice" Sixth".

Cart" "Fill in a vulnerable child form", on gut
"Articulating" "quantify". - That" al "Articulating" "quantify": - That au you vo got-. "Note going to steed op in court" / Delve" / Dustily/ Evidence - "Toys" " Foebual. search - / unexplained - explained/ "Any one thing feel, her, - "Foetual" - "see somebody is Suffering; (tnvironment/Bruise - immobile l. Verbal/Clues Angina or druag/Picture on wan "Justifiable"

(IDan't think it's all in the Gut'.) rental issue -call for mem.

* Gut/intulión

* Supported by evidence

* Difficulties completing saleguording farms on gut.

284 


\section{Appendix 4: Questionnaire}

Paramedic Attitudes \& Confidence Levels Towards Their Role In Child Protection

Thanks for taking the time to look at this survey

If you continue to complete this survey, your consent will be assumed.

All entries are completely anonymous.

Please DO NOT complete this survey if you are NOT a HCPC registered paramedic or DO NOT work for $x \times x \times x x x x x x x x x x$ AMBULANCE SERVICE.

If you would be interested in taking part in a focus group also, please view the details at the end of this survey.

Thank you for your time.

(C) James Michael Brady (2014). Use of this subject specific data collection tool, and the results thereof for research, teaching, or private study purposes, and or any substantial or systematic reproduction, redistribution, reselling, loan, sub licensing, systematic supply, or distribution in any form to anyone, without previous written consent from the author (who owns such intellectual property), is expressly forbidden. 


\section{Safequarding In Practice}

1. When Was Your Last Child Protection Training Update?
Within the Last Six Months
Within the Last Year
Within the Last Two Years
Within the Last Three Years
Within the Last Four Years
More than Four Years ago

2. Within the Last Three Years, About How Many Child Protection Referrals Have You Made (i.e. Vulnerable children form online, or via clinical desk, to Social Service or The Police (direct or indirect)?

10 or less

$10-20$

21-30

30 or more

3. I Have Received Adequate Child Protection Training To Ensure I Can Practice Safely
Strongly
Neither Agree or
Agree
Agree
Disagree
Disagree
Strongly
Disagree

4. I Reqularly Get Feedback From The Child Protection Referrals I Have Submitted
Strongly
Neither Agree or
Strongly
Agree
Agree
Disagree
Disagree
Disagree

5. I Feel My Child Protection Referrals Are Taken Seriously By The Receiving Departments

Strongly

Neither Agree or

Agree

Agree

Disagree

Disagree

Strongly

Disagree 


\section{Patterns Of Knowing}

Please tick as appropriate:

6. When deciding on whether to complete a Child Protection referral, how often do you use factual data, such as that gained from training and education? (CPD, university) and or that gained from your physical assessment (i.e. Signs, Symptoms, \& Observations)
All The
Time
Often
Not Sure
Not Often
Not
At All

7. When deciding on whether to complete a Child Protection referral, how often do you use intuition, "gut feelings", or draw from your previous clinical or life experiences?
All The
Time
Often
Not Sure
Not Often

8. When deciding on whether to complete a Child Protection referral, how often does your own ethical judgment play a role? (i.e. what you yourself know and think is right; influenced by your own beliefs or belief system)

All The

Time

Often

Not Sure

Not Often

9. When completing a Child Protection referral, how often do you recognise (and think about) your own personal knowledge of yourself? (i.e. your own abilities to empathize, your possible prejudices, and attitudes towards the situation)

All The

Time
Often 


\section{Attitudes Towards Safequarding Children \& Young People}

Please rate how relevant you think these subjects are to Your Role As A

Paramedic in relation to child protection:

\section{Knowledge of Physical Abuse}

Highly Relevant Relevant Neither Relevant or Not Relevant Not Relevant Not at all Relevant

\section{Knowledge of Emotional Abuse}

Highly Relevant Relevant Neither Relevant or Not Relevant Not Relevant Not at all Relevant

\section{Knowledge of Sexual Abuse}

Highly Relevant Relevant Neither Relevant or Not Relevant Not Relevant Not at all Relevant

\section{Knowledge of Neglect}

Highly Relevant Relevant Neither Relevant or Not Relevant Not Relevant Not at all Relevant

\section{Knowledge of the psychological \& sociological factors affecting child} and family health (i.e. how poverty, class structure \& mental health issues affect child development).

Highly Relevant Relevant Neither Relevant or Not Relevant Not Relevant Not at all Relevant

15. Knowledge of health promotion and disease prevention in relation to child \& family health.

Highly Relevant Relevant Neither Relevant or Not Relevant Not Relevant Not at all Relevant

16. Knowledge of life span development (i.e. The Physical, Cognitive, and Psychosocial developmental landmarks a Child is Expected to Make).

Highly Relevant Relevant Neither Relevant or Not Relevant Not Relevant Not at all Relevant 


\section{Paramedics' Levels of Confidence}

Please rate your agreement with the following statement:

17. On reflection, with hindsight, and with experience, I recall times where Child Protection referrals should have been completed but were not. (All Questionnaires are Anonymous).

Agree Disagree

18. Please indicate any reasons as to why Child Protection referrals may have not been completed in practice (All Questionnaires are Anonymous).

\begin{tabular}{|l|l|l|}
\hline Lack of confidence & $\begin{array}{l}\text { Not stood down to make a } \\
\text { referral }\end{array}$ & $\begin{array}{l}\text { Wanted to avoid } \\
\text { confrontation }\end{array}$ \\
\hline $\begin{array}{l}\text { Afraid of outcome if I } \\
\text { was wrong }\end{array}$ & $\begin{array}{l}\text { Inadequate computer / internet } \\
\text { provisions }\end{array}$ & I never get any feedback \\
\hline $\begin{array}{l}\text { Not enough time on } \\
\text { shift }\end{array}$ & $\begin{array}{l}\text { Poor knowledge of abuse and } \\
\text { neglect }\end{array}$ & $\begin{array}{l}\text { Paramedic referrals are not } \\
\text { taken seriously }\end{array}$ \\
\hline Not my responsibility & $\begin{array}{l}\text { Felt intimidated by parents / } \\
\text { caregivers }\end{array}$ & Had a suspicion but no proof \\
\hline $\begin{array}{l}\text { Realised too late after } \\
\text { the job had closed }\end{array}$ & $\begin{array}{l}\text { I only have to report physical, } \\
\text { sexual, emotional abuse or } \\
\text { neglect }\end{array}$ & None of these options \\
\hline
\end{tabular}

19. If other reasons apply then please state below (All Questionnaires are Anonymous):

Other Possible Reason

Other Possible Reason

Other Possible Reason 


\section{Confidence In Decision Making}

Please rate your levels of CONFIDENCE in the following areas:

20. My ability to recognise signs of Physical Abuse

Very Confident Confident Neither Confident or Not Confident Not Confident Not al all Confident

21. My ability to recognise signs of Emotional Abuse

Very Confident Confident Neither Confident or Not Confident Not Confident Not all Confident

22. My ability to recognise signs of Sexual Abuse

Very Confident Confident Neither Confident or Not Confident Not Confident Not all Confident

23. My ability to recognise signs of Neglect

Very Confident Confident Neither Confident or Not Confident Not Confident Not all Confident

24. My ability to recognise signs of Child Sexual Exploitation

Very Confident Confident Neither Confident or Not Confident Not Confident Not all Confident

25. My ability to recognise signs / risk factors of Child and Young Female Genital Mutilation

Very Confident Confident Neither Confident or Not Confident Not Confident Not all Confident

26. My knowledge of the effects poor housing can have on child development (damp, overcrowding, excess noise),

Very Confident Confident Neither Confident or Not Confident Not Confident Not all Confident

27. My knowledge of the effects poor levels of social interaction can have on child development (Lack of group play, \& financial exclusion from clubs or social events)

Very Confident Confident Neither Confident or Not Confident Not Confident Not all Confident

28. My knowledge of the effects that poor diet has on child development \& educational attainment.

Very Confident Confident Neither Confident or Not Confident Not Confident Not at all Confident 
29. My knowledge of the signs that a child may not be achieving their expected physical, cognitive, and psychosocial developmental landmarks.

Very Confident Confident Neither Confident or Not Confident Not Confident Not all Confident

\section{About You}

30. Are you male or female?

Male

Female

31. What is your age?

1820

2129

3039

4049

5059

60 or older

32. With which race to you most closely identify?

White or white European

Black or African American

Indian or American Indian

Asian

Native Hawaiian or other Pacific Islander

From multiple races

Some other race (please specify)

33. With which religion (if any) do you most closely identify?

Protestantism

Catholicism

Christianity

Judaism

Islam

Buddhism

Hinduism

Native American

Inter/Nondenominational

Prefer Not To Say

No Religion 
34. What is the highest level of education you have completed that is related to paramedicine?

IHCD (Institute of Health Care Development) paramedic certificate

Diploma in higher education and or foundation degree

Bachelor's degree without honours

Bachelor's degree with honours

Post graduate certificate

Masters' degree

Professional doctorate, doctorate, or PhD.

35. About how long have you been qualified as a Paramedic?

$0-2$ Years

2-4 Years

4-6 Years

6-8 Years

8-10 Years

10-12 Years

12-14 years

14 Years or More

36. About how long has your length of service been (combined all ambulance services and all roles; i.e. Patient Transport, Technician)?

$0-2$ Years

2-4 Years

4-6 Years

6-8 Years

8-10 Years

10-12 Years

12-14 years

14 Years or More

37. What is your current role within the Ambulance Service?

Critical Care Paramedic

Dual Qualified Nurse \& Paramedic

Paramedic Practitioner / Emergency Care Practitioner / Advanced Paramedic

Paramedic / Paramedic Clinical Advisor / Supervisor / Paramedic Trainer / officer / or any other senior role

38. Please select which box best describes your paramedic career journey 
- Emergency / Ambulance care assistant to IHCD technician to diploma, foundation, or honours degree paramedic

- Emergency / Ambulance care assistant to IHCD technician to IHCD paramedic

- IHCD paramedic who continued to gain a paramedic related diploma, foundation, or honours degree

- High School / college, straight to Honours degree paramedic

- Emergency care assistant to IHCD technician, to diploma, foundation, or honours degree paramedic

- IHCD technician to IHCD paramedic

- Emergency care assistant to IHCD technician to IHCD paramedic

- IHCD technician to diploma, foundation, or honours degree paramedic

- High School / college, to diploma, foundation, or honours degree paramedic

39. Which, if any, occupational areas have you worked within prior to paramedicine?

\begin{tabular}{|l|l|l|}
\hline Construction and Extraction & $\begin{array}{l}\text { Installation, } \\
\text { Maintenance, and } \\
\text { Repair }\end{array}$ & $\begin{array}{l}\text { Sales and Related } \\
\text { Occupations }\end{array}$ \\
\hline $\begin{array}{l}\text { Healthcare Practitioners and } \\
\text { Technical }\end{array}$ & $\begin{array}{l}\text { Management } \\
\text { Occupations }\end{array}$ & $\begin{array}{l}\text { Food Preparation and } \\
\text { Serving Related }\end{array}$ \\
\hline $\begin{array}{l}\text { Transportation and } \\
\text { Materials Moving }\end{array}$ & $\begin{array}{l}\text { Farming, Fishing, and } \\
\text { Forestry }\end{array}$ & $\begin{array}{l}\text { Community and Social } \\
\text { Service }\end{array}$ \\
\hline $\begin{array}{l}\text { Architecture and } \\
\text { Engineering }\end{array}$ & $\begin{array}{l}\text { Computer and } \\
\text { Mathematical }\end{array}$ & $\begin{array}{l}\text { Arts, design, } \\
\text { entertainment, sports, and } \\
\text { media occupations }\end{array}$ \\
\hline $\begin{array}{l}\text { Office and Administrative } \\
\text { Support }\end{array}$ & $\begin{array}{l}\text { Healthcare Support } \\
\text { Occupations }\end{array}$ & $\begin{array}{l}\text { Food Preparation and } \\
\text { Serving Related }\end{array}$ \\
\hline $\begin{array}{l}\text { Maintenance Occupations } \\
\text { Farming, Fishing, and } \\
\text { Forestry }\end{array}$ & $\begin{array}{l}\text { Education, Training, and } \\
\text { Library }\end{array}$ \\
\hline $\begin{array}{l}\text { Community and Social } \\
\text { Service }\end{array}$ & $\begin{array}{l}\text { Life, Physical, and } \\
\text { Social Science }\end{array}$ & Production Occupations \\
\hline $\begin{array}{l}\text { Protective Service } \\
\text { Occupations }\end{array}$ & Legal Occupations & $\begin{array}{l}\text { Personal Care and } \\
\text { Service Occupations }\end{array}$ \\
\hline & & NONE OF THE OPTIONS \\
\hline
\end{tabular}




\section{Thank you for your time}

Your contribution will assist in developing evidence bases within paramedicine

If you would like to be involved in further focus group research related to the role paramedics play in child protection, please email the primary researcher, Mike Brady at stating your name, educational route, current clinical grade, and work location. Numbers are limited and so participants will be accepted on a first come first serve basis within each geographical and demographic category.

Again thank you for your time. 


\section{Appendix 5: Advertisements for questionnaire participation}

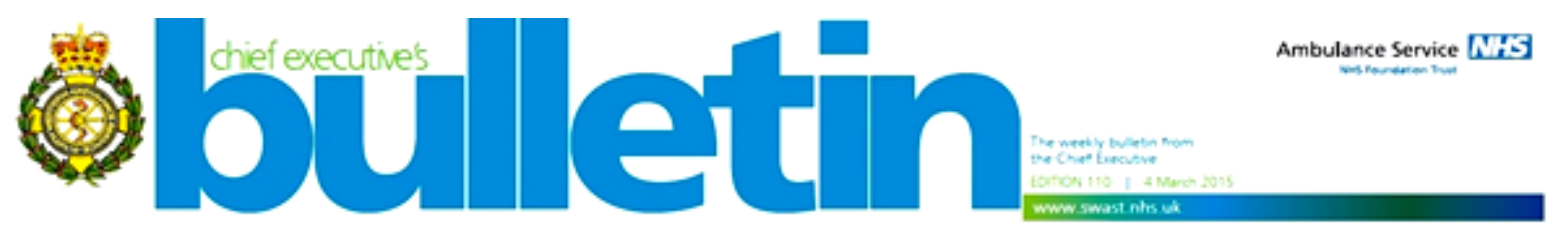

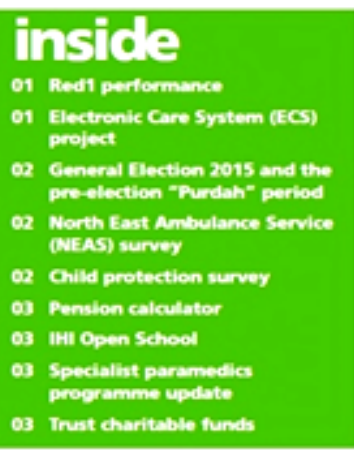

inside

01 Redi pertormance

o1 Electronic Care System (ECS)

c2 Ceneral Election 2015 and the

North East ing

2 Child protection survey

as Pension calculater

es Specialiht paramedica

03 Inut charituble thunds

\section{Child protection survey}

Mike Brady, a Clinical Supervisor on the Clinical Support Desk in , is studying for his PhD, and as part of this doctorate he is undertaking original research. His research area is The Investigation of Paramedics

Thoughts, Attitudes and Perceptions of their Role in Relation to Child Protection.

If you are an $\mathrm{HCPC}$ registered paramedic and have 10 minutes to spare, he would be grateful if you could complete his anonymised questionnaire by following the link below. Your contribution will add to the data in an important and under-researched area of practice

You can complete the questionnaire at: wrww.surveymonkey.com/r/JN7VXCV.

\section{RECENT ACTIVITY}

\section{Right Care shared a link.}

\section{Tiplace}

time

10 March at 10:48 \& 2 .

Hi everyone, thanks to every paramedic that has done so far, but can I ask any of those who haven't and might have 5-10 mins to spare (who are SWAST \& HCPC Registered PARAMEDICS), to complete this questionnaire on Child Protection

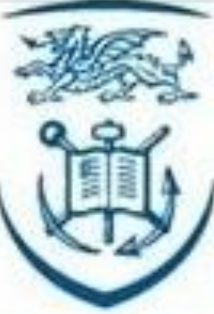

ansea Univer: fysgol Aberta

\section{Paramedic Attitudes \& Confidence Levels Towards Their Role In Child Protection Survey}

Web survey powered by Surveymonkey.com.Create your own online survey now with Surveymonkey's...
Like comment Share 


\title{
Appendix 6: Information sheets for focus groups
}

\author{
Information Sheet for focus group participants \\ An Investigation of Paramedics' attitudes and understanding in relation to the \\ safeguarding of children in their daily practice
}

Please may I introduce myself? My name is Mike Brady - I am a registered paramedic, lecturer in emergency and unscheduled care, an academic author, and a $\mathrm{PhD}$ student researching paramedics' thoughts and feelings in relation to safeguarding children and young people. I would be most grateful if you would read this information sheet and consider taking part in this.

What is the purpose of this study? This study is to find out from participants within the Ambulance Service NHS Trust, the attitudes, perceptions, and confidence levels in decision making of paramedics when it comes to child and young person safeguarding. The aim is to use the findings to improve or validate future educational and training provisions.

Who is funding the study and who is involved? I am funding this study, with no financial funding from any external organisation. This is with the support however of the College of Human and Health Sciences Swansea University, and the Ambulance Service, who are providing the loan of computer software, recording devices and the use of trust buildings, in which to safely conduct the research.

Why have I been asked to take part in this research? You have been chosen because you are a Health Care Professions Council (HCPC) registered paramedic, whose thoughts, attitudes, and perceptions I would like to investigate.

Do I have to take part? No, it is up to you to decide whether or not to take part. I would like to assure you that if you decide not to it will not have any effect on your role as a HCPC registered paramedic within the Ambulance Service.

What will happen if I decide to take part in the study? If you feel satisfied that you have been given enough information about the study and would like to take part, you will be asked to sign a consent form, when focus groups have been arranged. You are free to withdraw at any time and without giving any reason. If you withdraw, it will not affect your role within as a HCPC registered paramedic within the Ambulance Service. 
What will happen to me if I take part? You will be asked to attend one focus group with other Ambulance Service paramedics. This will take place at an Ambulance Service building local to you, and take $60-90$ minutes in all. The focus group discussions will be recorded and the interview will then by typed-up. We will ensure any identifying information, including your name, is removed from the typed-up notes. Any names will be substituted with case numbers. We will ask you to consent to taking part and sign the consent form which is shown at the end of this information sheet.

Will my taking part in this study be kept confidential? We will follow ethical and legal practice and all the information about you will be held in confidence, unless deliberately unsafe practice is uncovered. All data will be stored securely in line with best research practice, and the data protection act 1998.

In what situations will the HCPC be contacted, if any? The Health Care Professions Council will NOT be contacted following this research as a result of participants' perceptions, attitudes, or levels of confidence towards child safeguarding or if training and education needs are highlighted. However the researcher as a HCPC registered clinician himself and a health science researcher is bound to report any deliberately unsafe practice falling short of the HCPC conduct Standards of conduct, performance and ethics.

Payment. I am afraid we are not able to make any financial payment to you for taking part, but as you will be given a £10 shopping voucher to reimburse travelling expenses.

What are the possible benefits of taking part? You will find out the views, perceptions, attitudes and confidence levels of paramedics such as yourself, as well as having an opportunity to share your views and experiences. Findings from this study will be support the need to improve or validate current education and training for paramedics.

Are there any disadvantages if I do not take part? There are no disadvantages to you if you do not take part. You may withdraw at any time.

Is there anyone I can talk to if I feel I need to, as a result of the feelings and emotions raised by participating in this research?

Ambulance Service staff have access to 24 hour online and telephone counselling and psychological support services provided by www.first-assist.com for a range of issues including but not limited to work or home life trauma, stress, anxiety, separation / divorce, and bereavement. 
The telephone number is 0800716017 , and the online access code you type into both the login and password boxes on the first-assist online portal is 72840 .

In addition civilian services are also available:

The Survivors Trust: 01788550554 \& info@thesurvivorstrust.org

The Samaritans: 08457909090 \& jo@ samaritans.org

Has this study received ethical approval? This study has been approved by the College of Human and Health Science, Ethics Committee, Swansea University, and the Ambulance Service.

Who can I contact about this study? Please contact me at anytime or

Thank you very much for considering taking part in this study. I look forward to hearing from you 


\section{Appendix 7: Consent forms}

\section{CONSENT FORM}

\section{An Investigation of Paramedics' attitudes and understanding in relation to the safeguarding of children in their daily practice}

Chief Investigator: Mike Brady, College of Human and Health Science, Swansea University, Swansea SA2 8PP.

Email:

Please initial the statements below to signify your consent (initial those you consent to)

1. I confirm that I have read and understood the information sheet for the above study and have had the opportunity to ask questions. (initial)

2. I agree to take part in the above study as a participant in an audio-taped focus group interview. (initial)

3. I understand that my participation is voluntary and that I am free to withdraw at any time, without giving any reason. (initial)

4. I understand that the information gathered during the course of the audio-taped focus group interview will be confidential and anonymized. (initial)

5. I understand that information from of the audio-taped focus group interview will be transcribed (typed up), rendered anonymous and will be read and analyzed by members of the research team. I give permission for the team members to have access to this information. (initial)

6. I agree to the data being used for publication (in whole or in part) in peer-reviewed academic and professional journals. (initial)

Name of Participant

Date

Signature

Age: Years of service as a paramedic:

Email: 